\title{
Kinetik der Reaktionen des Hydroxylradikals mit ungesättigten Kohlenwasserstoffen in einer Lavaldüsenexpansion
}

\author{
Dissertation \\ zur Erlangung des Doktorgrades \\ der Mathematisch-Naturwissenschaftlichen Fakultäten \\ der Georg-August-Universität zu Göttingen
}

vorgelegt von

Tim Spangenberg (geb. Knöppel)

aus Kassel

Göttingen 2003 
D7

Referent: Prof. Dr. J. Troe

Korreferent: Prof. Dr. K.-H. Hoyermann

Tag der mündlichen Prüfung: 05.11.2003 
Für Yvonne 

Nicht weil die Dinge schwierig sind, wagen wir sie nicht, sondern weil wir sie nicht wagen, sind sie schwierig.

M. L. Möller 



\section{Zusammenfassung}

Die Untersuchung der Reaktionen des Hydroxylradikals mit ungesättigten Kohlenwasserstoffen (Ethin, Ethen, Propen und Isopren) bei tiefen Temperaturen spielt für das Verständnis der Chemie der Troposphäre eine bedeutende Rolle. Aus der Temperaturabhängigkeit der Geschwindigkeitskonstanten können zunächst Informationen über die mechanistischen Details der Reaktionen abgeleitet werden. Dabei sind bimolekulare Geschwindigkeitskonstanten empfindlich auf Details der Potentialfläche in chemisch relevanten Energiebereichen.

In der vorliegenden Arbeit werden experimentelle Untersuchungen der Reaktionen des Hydroxylradikals $(\mathrm{OH})$ mit verschiedenen ungesättigten Kohlenwasserstoffen bei tiefen Temperaturen präsentiert. Die Abkühlung der Reaktanden auf bis zu $58 \mathrm{~K}$ erfolgte durch die Expansion in einer Lavaldüse. Die Lavaldüse erzeugt einen in Temperatur, Dichte und Machzahl konstanten Strahl aus einem Trägergas $\left(\mathrm{N}_{2}\right)$. OHRadikale werden durch Laserphotolyse von Wasserstoffperoxid $\left(\mathrm{H}_{2} \mathrm{O}_{2}\right)$ bei $193 \mathrm{~nm}$ und $248 \mathrm{~nm}$ erzeugt. Die zeitliche Entwicklung der Hydroxylradikalkonzentration in einem Überschuss des Reaktionspartners wurde mit Hilfe der laserinduzierten Fluoreszenz (LIF) gemessen.

Begleitend zu den experimentellen Studien wird eine Abschätzung der bestimmten Geschwindigkeitskonstanten nach dem Modell der statistischen adiabatischen Reaktionskanäle (SACM) durchgeführt. Die Eigenschaften der Edukte und Produkte wurden mit Hilfe von DFT-Methoden berechnet.

Die bestimmten Geschwindigkeitskonstanten für die Reaktionen des Hydroxylradikals mit ungesättigten Kohlenwasserstoffen zwischen 60 und $300 \mathrm{~K}$ erstrecken sich über zwei Größenordnungen zwischen $10^{-12}$ und $10^{-10} \mathrm{~cm}^{3} \mathrm{~s}^{-1}$. Dabei ist eine systematische Zunahme der Reaktionsgeschwindigkeiten von Ethin nach Ethen, Propen und Isopren $\mathrm{zu}$ erkennen. Weiterhin wird für alle OH-Alken-Reaktionen (zumindest bis $100 \mathrm{~K}$ ) eine negative Temperaturabhängigkeit der Geschwindigkeitskonstanten beobachtet. Diese Tatsachen können durch die Annahme von langreichwei- 
tigen Multipol-Wechselwirkungen und den damit verbundenen weit außen liegenden Übergangszuständen der Reaktionen erklärt werden.

Für die Reaktionen des OH-Radikals mit Ethin und Ethen wurden Hochdruckgrenzwerte der Reaktionen über die Schwingungsrelaxation des Hydroxylradikals ermittelt. Für die OH-Rekombinationsreaktion des Propen und besonders für Isopren wurde ein Absinken der Geschwindigkeitskonstanten bei Temperaturen unterhalb von $100 \mathrm{~K}$ gefunden. Ein Effekt, der möglicherweise mit dem Vorhandensein von intermediären Isomeren erklärt werden kann.

Die Analyse zeigt, daß die Reaktion $\mathrm{OH}+$ Ethin eine deutliche Barriere besitzt, die die geringe Temperaturabhängigkeit im Bereich zwischen 300 und $60 \mathrm{~K}$ dominiert. Die Reaktion von $\mathrm{OH}$ und Ethen zeigt über den gesamten gemessenen Temperaturbereich eine negative Temperaturabhängigkeit. 


\section{Inhaltsverzeichnis}

1 Einleitung 1

2 Theoretische Grundlagen 17

2.1 Unimolekulare Reaktionsdynamik . . . . . . . . . . . . . 17

2.1.1 Frühe Theorien . . . . . . . . . . . . . . . . 17

2.1.2 Die RRKM-Theorie . . . . . . . . . . . . . . . . . 22

2.1.3 Das SAC-Modell . . . . . . . . . . . . . . . . 23

2.1.4 Die Phasenraumtheorie (PST) . . . . . . . . . . . 27

2.2 Bestimmung von Molekülparametern . . . . . . . . . . . . . . . 28

2.2.1 Hartree-Fock-Methode (HF-SCF) . . . . . . . . . . . . . 31

2.2.2 Störungstheorie $\left(\mathrm{MP}_{\mathrm{n}}\right) \ldots \ldots \ldots$. . . . . . . . . 31

2.2.3 Dichtefunktionaltheorie (DFT) . . . . . . . . . . 33

2.2.4 Basissätze . . . . . . . . . . . . . . . . . 34

2.3 Spektroskopie des OH-Radikals . . . . . . . . . . . . . . 36

2.3.1 Grundzustand $\left(X^{2} \Pi_{i}\right)$. . . . . . . . . . . . . 36

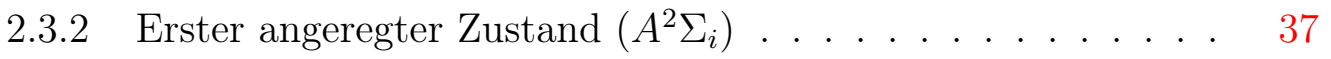

2.3.3 Laserinduzierte Fluoreszenz . . . . . . . . . . . . . . . 37

3 Experimenteller Teil 39

3.1 Überschallexpansion . . . . . . . . . . . . . . . . . . . . . 39

3.2 Lavaldüse . . . . . . . . . . . . . . . . . . . . . . . . . 43

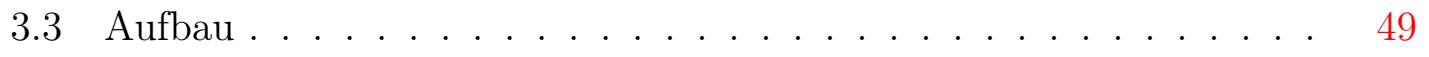

3.3.1 Vakuumkammer . . . . . . . . . . . . . . . . 49

3.3.2 Laser und optischer Aufbau . . . . . . . . . . . . . . 49

3.4 Chemische Substanzen . . . . . . . . . . . . . . . . 52

3.5 Signalaufnahme und Datenverarbeitung . . . . . . . . . . . 52

3.6 Zeitliche Steuerung . . . . . . . . . . . . . . 55

3.7 Charakterisierung der Expansion . . . . . . . . . . . 55 
4 Ergebnisse $\quad 65$

4.1 Relaxation des OH-Radikals . . . . . . . . . . . . . . . 65

4.2 Reaktionen des OH-Radikals . . . . . . . . . . . . . 68

4.2.1 Ethin $+\mathrm{OH} \ldots \ldots \ldots 71$

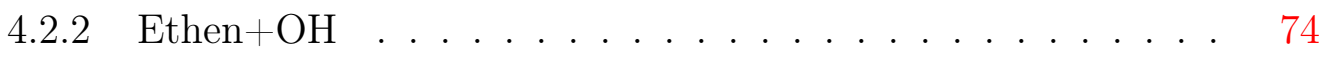

4.2 .3 Propen $+\mathrm{OH} \ldots \ldots \ldots 76$

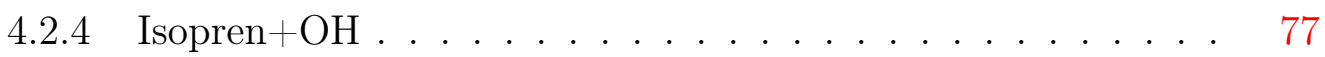

5 Diskussion $\quad 79$

5.1 Ethin $+\mathrm{OH} \ldots \ldots \ldots \ldots \ldots . \ldots \ldots$

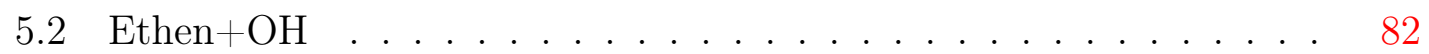

5.3 Propen $+\mathrm{OH} \ldots \ldots \ldots \ldots$. . . . . . . . . . . . . 85

5.4 Isopren $+\mathrm{OH} \ldots \ldots \ldots \ldots$. . . . . . . . . . . . . . . . . . . . . . . . . . . . . 88

5.5 Vergleich der untersuchten Reaktionen . . . . . . . . . . . . 90

6 Ausblick 101

A Geometrien, Frequenzen und Energien 105

A.1 Hydroxylradikal . . . . . . . . . . . . . . . . . 106

A.1.1 Geometrie . . . . . . . . . . . . 106

A.1.2 Normalmoden . . . . . . . . . . . . . 106

A.1.3 Energien . . . . . . . . . . . . . . . 107

A.2 Ethin . . . . . . . . . . . . . . . . . . 108

A.2.1 Geometrie ................... . . 108

A.2.2 Normalmoden . . . . . . . . . . . . . . . . . 108

A.2.3 Energien . . . . . . . . . . . . . . . . 109

A.3 Hydroxyethinylradikal . . . . . . . . . . . . . . . . . . 110

A.3.1 Geometrie . . . . . . . . . . . . . . . 110

A.3.2 Normalmoden . . . . . . . . . . . . . . . . . . 111

A.3.3 Energien . . . . . . . . . . . . . . . 111

A.4 Ethen . . . . . . . . . . . . . . . . . . . . . 112

A.4.1 Geometrie ................... 112

A.4.2 Normalmoden . . . . . . . . . . . . . . . . 113

A.4.3 Energien . . . . . . . . . . . . . . . . 113

A.5 Hydroxyethenylradikal . . . . . . . . . . . . . . . . . . . . . . . . . . . . . . . . . 114

A.5.1 Geometrie .................. . 114

A.5.2 Normalmoden ................... . . 114

A.5.3 Energien . . . . . . . . . . . . . . . 116

A.6 Relative Energien, Reaktionsenthalpien . . . . . . . . . . . . 117 
B SACM-Rechnungen 119

B.1 Korrelation der Oszillatoren . . . . . . . . . . . . . . . . . . . 119

B.1.1 Ethin $+\mathrm{OH} \ldots \ldots \ldots 120$

B.1.2 Ethen $+\mathrm{OH} \ldots \ldots \ldots . \ldots . \ldots 121$

B.2 Parameter für die SACM-Rechnungen . . . . . . . . . . . . . . . . 122

B.2.1 Ethin $+\mathrm{OH} \ldots \ldots \ldots \ldots \ldots$. . . . . . . . . . . . . . . . . . . . . . . . . . . . . . 123

B.2.2 Ethen $+\mathrm{OH} \ldots \ldots \ldots \ldots$

$\begin{array}{ll}\text { Abbildungsverzeichnis } & 125\end{array}$

$\begin{array}{ll}\text { Literaturverzeichnis } & 127\end{array}$ 



\section{Kapitel 1}

\section{Einleitung}

Um die Relevanz der in dieser Arbeit untersuchten Reaktionen von Ethin $\left(\mathrm{C}_{2} \mathrm{H}_{2}\right.$, auch Acetylen), Ethen $\left(\mathrm{C}_{2} \mathrm{H}_{4}\right)$, Propen $\left(\mathrm{C}_{3} \mathrm{H}_{6}\right)$ und 2-Methyl-1,3-butadien $\left(\mathrm{C}_{5} \mathrm{H}_{8}\right.$, auch Isopren) mit Hydroxylradikalen $(\mathrm{OH})$ bei tiefen Temperaturen zu verdeutlichen, werden zunächst die Moleküle und deren Reaktionen vorgestellt und anschließend die Bedeutung der Untersuchung der Reaktionskinetik bei tiefen Temperaturen verdeutlicht.

\section{Das OH-Radikal}

Die Atmosphäre der Erde stellt ein oxidierendes Medium dar. Viele wichtige Spurengase in der Atmosphäre werden durch oxidative Prozesse vernichtet, so z. B. Treibhausgase wie Methan $\left(\mathrm{CH}_{4}\right)$, Kandidaten für den stratosphärischen Ozonabbau (FCKW's) oder giftige Verbrennungsgase wie Kohlenmonoxid (CO). Zu den am häufigst vorhandenen Oxidantien gehören molekularer Sauerstoff $\left(\mathrm{O}_{2}\right)$ und Ozon $\left(\mathrm{O}_{3}\right)$. Aufgrund ihrer relativ großen Bindungsenergien sind sie jedoch weitestgehend ungeeignet, unreaktive, nichtradikalische Spezies zu reduzieren, d.h. die entsprechenden Reaktionen verlaufen bis auf ein paar Ausnahmen verhältnismäßig langsam ab. Das Hydroxylradikal $(\mathrm{OH})$ hingegen spielt zusammen mit dem Nitratradikal $\left(\mathrm{NO}_{3}\right)$ eine absolut zentrale Rolle in der troposphärischen Oxidationschemie nichtradikalischer Spezies. Ein Großteil der troposphärischen Oxidation oxidierbarer Gase am Tag findet durch einen direkten Angriff von Hydroxylradikalen statt, während Nitratradikale $\left(\mathrm{NO}_{3}\right)$ für oxidative Prozesse in der Nacht zuständig sind. Die hohe Reaktivität von Hydroxylradikalen besonders gegenüber Wasserstoff enthaltenden Molekülen läßt sich durch die leichte Wasserstoffatom-Abstraktion erklären, die das OH-Radikal in Wasser konvertiert.

Ausgangspunkt der größten Quelle von OH-Radikalen ist die Ozon-Photolyse bei Wellenlängen kleiner als $336 \mathrm{~nm}$, bei der elektronisch angeregter Singulett-Sauerstoff 
$\left(\mathrm{O}\left({ }^{1} \mathrm{D}\right)\right)$ entsteht. Aus der Troposphäre gelangt Wasserdampf in die Stratosphäre, wo er nach den Reaktionen 1.1 bis 1.3 mit Singulett-Sauerstoff Hydroxylradikale bildet. Da die Konzentration an Wasserdampf mit steigender Höhe abnimmt, nimmt gleichzeitig die Bedeutung von OH-Radikalen für die Atmosphärenchemie ab.

$$
\begin{aligned}
\mathrm{O}_{3}+\mathrm{h} \nu & \longrightarrow \mathrm{O}_{2}+\mathrm{O}\left({ }^{1} \mathrm{D}\right) \\
\mathrm{O}\left({ }^{1} \mathrm{D}\right)+\mathrm{M} & \longrightarrow \mathrm{O}+\mathrm{M} \\
\mathrm{O}\left({ }^{1} \mathrm{D}\right)+\mathrm{H}_{2} \mathrm{O} & \longrightarrow 2 \mathrm{OH}
\end{aligned}
$$

Aus den Gleichungen 1.1 bis 1.3 läßt sich eine einfache Gleichung für die Bildungsgeschwindigkeit der Hydroxylradikale bei Annahme einer kleinen, gleichbleibenden Singulett-Sauerstoffkonzentration ableiten. Mit Hilfe von Laborexperimenten wurde gezeigt, dass Reaktion 1.2 sehr viel schneller verläuft als Reaktion 1.3. Somit ergibt sich folgender Zusammenhang:

$$
\begin{aligned}
\frac{\mathrm{d}[\mathrm{OH}]}{\mathrm{d} t}=2 k_{3}\left[\mathrm{O}\left({ }^{1} \mathrm{D}\right)\right]\left[\mathrm{H}_{2} \mathrm{O}\right] & =\frac{2 k_{1} k_{3}}{k_{2}[\mathrm{M}]+k_{3}\left[\mathrm{H}_{2} \mathrm{O}\right]}\left[\mathrm{O}_{3}\right]\left[\mathrm{H}_{2} \mathrm{O}\right] \\
& \approx \frac{2 k_{1} k_{3}}{k_{2}[\mathrm{M}]}\left[\mathrm{O}_{3}\right]\left[\mathrm{H}_{2} \mathrm{O}\right]
\end{aligned}
$$

Zusätzliche, jedoch wesentlich unbedeutendere Quellen für Hydroxylradikale sind die Photolyse von gasförmiger salpetriger Säure (HONO) $(\lambda<400 \mathrm{~nm})$ und Wasserstoffperoxid $\left(\mathrm{H}_{2} \mathrm{O}_{2}\right)(\lambda<370 \mathrm{~nm})$. Die Lebensdauer von OH-Radikalen in der Troposphäre liegt im Bereich von wenigen Sekunden, was im Vergleich zu anderen Spurengasen (z. B. Wasserdampf: 13 Tage, $\mathrm{CH}_{3} \mathrm{Br}$ : 2 Jahre, $\mathrm{CH}_{4}$ : 9 Jahre) sehr kurz ist. Aufgrund dieser kurzen Lebensdauer sind die atmosphärischen Konzentrationen sehr variabel, liegen aber im Bereich von $10^{6} \mathrm{~cm}^{-3}$.

\section{Ungesättigte Kohlenwasserstoffe}

Trotz dieser geringen Konzentration ist es das Hydroxylradikal, welchem die Hauptarbeit bei der Oxidation von sogenannten „flüchtigen organischen Verbindungen“ (VOCs für volative organic compounds) zukommt. Zu den VOCs zählen prinzipiell alle mehr oder weniger flüchtigen organischen Spezies wie einfache Kohlenwasserstoffe, aber auch Aldehyde oder Ketone. Aufgrund seiner relativ langsamen Oxidation in der Troposphäre zählt Methan nicht zu den VOCs, die deshalb auch oft als „organische nicht-Methan Verbindungen“ (NMOCs für non-methane organic 
compounds) oder auch als „reaktive organische Gase“ (ROGs für reactive organic gases) bezeichnet werden.

Die globale Emission von VOCs fügt sich in die Reihe von Kohlenmonoxid (CO), Stickoxiden $\left(\mathrm{NO}_{x}\right)$ und Methan $\left(\mathrm{CH}_{4}\right)$ ein, wobei der Anteil an anthropogenen Quellen bei VOCs gegenüber biogenen Quellen eher im Hintergrund steht. Etwa zwei Drittel der gesamten VOCs stammen aus biogenen Quellen, dabei findet die Emission größtenteils von Bäumen, Sträuchern und Anbaupflanzen statt. Ethen z. B. ist ein Pflanzenhormon, das mit einer Geschwindigkeit von einigen Teragramm pro Jahr emittiert wird [1]. Bei mechanischer Belastung, erhöhter Temperatur oder Wassermangel der Pflanze kann die Emission von Ethen um ein bis zwei Größenordnungen ansteigen. VOCs wurden außerdem in Meerwasser und der darüber befindlichen Luft gefunden, darunter auch Ethen $\left(\mathrm{C}_{2} \mathrm{H}_{4}\right)$ und Ethin $\left(\mathrm{C}_{2} \mathrm{H}_{2}\right)[2,3,4,5]$.

Die Emission von Propen ist fast ausschließlich anthropogenen Ursprungs, in der Atmosphäre bildet es hochgiftige Aldehyde [6]. Nach Methan ist Isopren (2-Methyl1,3-Butadien) der zweithäufigste Kohlenwasserstoff in der Atmosphäre. Seine Anwesenheit beruht hauptsächlich auf der Emission von Pflanzen. Bei hoher Temperatur oder Wassermangel setzen einige Pflanzen Isopren frei, dies dient der Stabilisierung der Membranen von Pflanzenzellen [7]. Weltweit wird die Emission von Isopren durch Pflanzen tagsüber auf $300-450 \cdot 10^{12} \mathrm{~g} / \mathrm{Jahr}$ geschätzt, zwei Prozent des verfügbaren Kohlenstoffs der Photosynthese wird im Sommer als Isopren gebunden [8]. Durch die schnelle Reaktion des Isoprens mit Hydroxylradikalen ist seine Lebensdauer mit 1.5 bis 3 Stunden relativ gering. Die Oxidationsfähigkeit der Troposphäre wird zum überwiegenden Teil durch Methan und Isopren bestimmt, weiterhin spielt Isopren eine entscheidende Rolle bei der Bildung von troposphärischem Ozon.

Der Mechanismus der Oxidation verschiedener Klassen von VOCs durch Hydroxylradikale unterscheidet sich je nach Art der Komponente. Eine gute Übersicht über die Reaktionen des Hydroxylradikals mit verschiedenen Alkanen, Alkenen, Alkinen und aromatischen Kohlenwasserstoffen findet sich in Ref. [9]. Während es sich bei der Oxidation von Alkanen durch OH-Radikale quasi immer um eine WasserstoffatomAbstraktion zur Bildung von thermodynamisch stabilem Wasser handelt, ist der primäre Pfad bei der Reaktion von Alkenen mit $\mathrm{OH}$ die Addition des Radikals an die Doppelbindung.

$$
\begin{gathered}
\mathrm{C}_{n} \mathrm{H}_{2 n}+\mathrm{OH} \rightleftharpoons \mathrm{C}_{n} \mathrm{H}_{2 n} \mathrm{OH}^{*} \\
\mathrm{C}_{n} \mathrm{H}_{2 n} \mathrm{OH}^{*}+\mathrm{M} \rightarrow \mathrm{C}_{n} \mathrm{H}_{2 n} \mathrm{OH}+\mathrm{M}
\end{gathered}
$$

Dabei wird ein intermediäres Addukt gebildet, welches entweder wieder in die Edukte zerfällt oder stabilisiert wird (Gl. 1.5 und 1.6). Dieser Mechanismus konn- 
te durch massenspektrometrische Untersuchungen einiger OH-Alkenaddukte bestätigt werden $[10,11]$. Die Rückreaktion und somit der Zerfall in die Edukte spielt allerdings nur bei geringen Drücken eine Rolle. Die Geschwindigkeitskonstanten der $\mathrm{OH}-A l k e n-R e a k t i o n e n$ sind deswegen verglichen mit den entsprechenden $\mathrm{OH}$ Alkan-Reaktionen meist um mehr als eine Größenordnung größer und liegen im Bereich von $10^{-11} \mathrm{~cm}^{3} \mathrm{~s}^{-1}$. Außerdem ist für viele $\mathrm{OH}-$ Alken-Reaktionen sowohl eine Temperatur- als auch eine Druckabhängigkeit bekannt, so dass eine verlässliche Extrapolation zu atmosphärisch relevanten Bedingungen ein genaues Verständnis der Mechanismen erfordert.

\section{$\mathrm{OH}+$ Ethen}

Die Reaktion von OH-Radikalen mit Ethen (Gl. 1.7) ist eine in einem weiten Temperatur- und Druckbereich gut untersuchte Reaktion, wobei sich die Untersuchungen auf Temperaturen über $200 \mathrm{~K}$ beschränken.

$$
\mathrm{C}_{2} \mathrm{H}_{4}+\mathrm{OH}+\mathrm{M} \longrightarrow \text { Produkte }+\mathrm{M}
$$

In bisherigen Studien wurden die Temperaturabhängigkeit über relativ kleine Bereiche $(210-500 \mathrm{~K})$ gemessen, Zwischenprodukte beobachtet [10] und von der Druckabhängigkeit der Geschwindigkeitskonstanten auf den oben genannten Reaktionsmechanismus (Gl. 1.5, 1.6) geschlossen [12, 13, 14, 15, 16, 17, 18, 19, 20, 21, 22, 23, 24, 25, 26, 27, 28, 29, 30, 31]. Studien bei höheren Temperaturen zeigen, dass die Abstraktion eines Wasserstoffatoms von Ethen und somit die Bildung von Wasser ab ca. $700 \mathrm{~K}[32,33,34,35,36,37,38]$ bei 1 atm eine Rolle spielt und im Bereich von Verbrennungstemperaturen der vorherrschende Reaktionskanal ist. In einem intermediären Temperaturbereich $(500 \mathrm{~K}-700 \mathrm{~K})$ stellt sich ein echtes thermodynamisches Gleichgewicht für Reaktion 1.5 ein, die Temperaturabhängigkeit der Gleichgewichtskonstanten konnte für die Bestimmung von Reaktionsenthalpien genutzt werden $[33,34,39,30]$. Die Berechnung von Geschwindigkeitskonstanten sowie Reaktionsbarrieren für Reaktion 1.7 anhand von theoretischen Modellen begann Anfang der 80er Jahre und hat bei heutiger Rechenleistung der Computer eine zufriedenstellende Genauigkeit erreicht [40, 41, 42, 34, 43, 44, 45, 46, 47, 48, 49, 50].

\section{$\mathrm{OH}+$ Ethin}

Der Abbau von Alkinen wie Ethin $\left(\mathrm{C}_{2} \mathrm{H}_{2}\right)$ ist analog zum Alken-Abbau durch die

primäre Addition des Hydroxylradikals an die Dreifachbindung, die Adduktbildung 
und die daraus resultierende Druckabhängkeit der Geschwindigkeitskonstanten geprägt.

$$
\mathrm{C}_{2} \mathrm{H}_{2}+\mathrm{OH}+\mathrm{M} \longrightarrow \text { Produkte }+\mathrm{M}
$$

In ersten Studien zu Reaktion 1.8 Anfang der 70er Jahre wurden vor allem CO und $\mathrm{C}_{2} \mathrm{HO}$ als Reaktionsprodukte bei Raumtemperatur identifiziert $[51,52,53]$ und erste Geschwindigkeitskonstanten bestimmt $[52,14,54,16]$. In einer dieser frühen Arbeiten wurde eine leichte positive Temperaturabhängigkeit der Geschwindigkeitskonstanten zwischen 210 und $460 \mathrm{~K}$ festgestellt [14], jedoch wurde keine Druckabhängigkeit der Geschwindigkeitskonstanten bei Drücken zwischen 1 und 500 Torr beobachtet. Dies änderte sich in den nachfolgenden Untersuchungen $[55,56,57,58]$, in denen von einer Druckabhängigkeit der Geschwindigkeitskonstanten auf eine Adduktbildung geschlossen wurde. Das entstandene Addukt $\mathrm{C}_{2} \mathrm{H}_{2} \mathrm{OH}^{*}$ kann entweder durch einen Stoßpartner M stabilisiert werden oder in die Edukte zerfallen (Gl. 1.9 und 1.10).

$$
\begin{aligned}
& \mathrm{C}_{2} \mathrm{H}_{2}+\mathrm{OH} \rightleftharpoons \mathrm{C}_{2} \mathrm{H}_{2} \mathrm{OH}^{*} \\
& \mathrm{C}_{2} \mathrm{H}_{2} \mathrm{OH}^{*}+\mathrm{M} \rightarrow \mathrm{C}_{2} \mathrm{H}_{2} \mathrm{OH}+\mathrm{M}
\end{aligned}
$$

In diesen und weiteren Studien [58,29,59,60,61,62] wurde das Erreichen des Hochdruckgrenzwertes bei Raumtemperatur für Drücke von über 200 Torr gemessen. Um die Enthalpie der Reaktion zu ermitteln, wurden temperaturabhängige Gleichgewichtskonstanten bestimmt [63]. Es ergab sich ein Wert von $-33.6 \mathrm{kcal} \mathrm{mol}^{-1}$, aus den Studien von Hippler et al. ergab sich ein Wert von $-34.9 \mathrm{kcal} \mathrm{mol}^{-1}$ [30]. Weiterhin wurden Energiebarrieren sowohl als Aktivierungsenergien aus ArrheniusAuftragungen (vgl. Abschnitt 2.1) gewonnen als auch berechnet [41].

Hochtemperaturuntersuchungen zwischen 900 und $1700 \mathrm{~K}[64,65,66,67,37]$ machten deutlich, dass in Verbrennungsprozessen, in denen Hydroxylradikale und Ethin involviert sind, die direkte Abstraktion eines Wasserstoffatoms laut Gl. 1.11 in Konkurrenz zur Adduktbildung tritt und diese vielleicht sogar ersetzt.

$$
\mathrm{C}_{2} \mathrm{H}_{2}+\mathrm{OH} \rightarrow \mathrm{C}_{2} \mathrm{H}+\mathrm{H}_{2} \mathrm{O}
$$

Der Anteil dieses zusätzlichen Reaktionskanals am Gesamtprozess hängt vom Druck ab. Den Untersuchungen von Hippler et al. [30] zufolge ist der Einfluss der Wasserstoffabstraktion bei Drücken von 1 bar ab einer Temperatur von ca. $800 \mathrm{~K}$ nicht mehr vernachlässigbar. Die Arbeit von Hippler et al. ist auch die einzige experimentelle Untersuchung, in der die Reaktion bei Drücken von bis zu 140 bar beobachtet wurde. Somit ergeben sich aus dieser Arbeit die verlässlichsten experimentellen Werte für die temperaturabhängige Hochdruckgeschwindigkeitskonstante, wobei die Arbeiten 

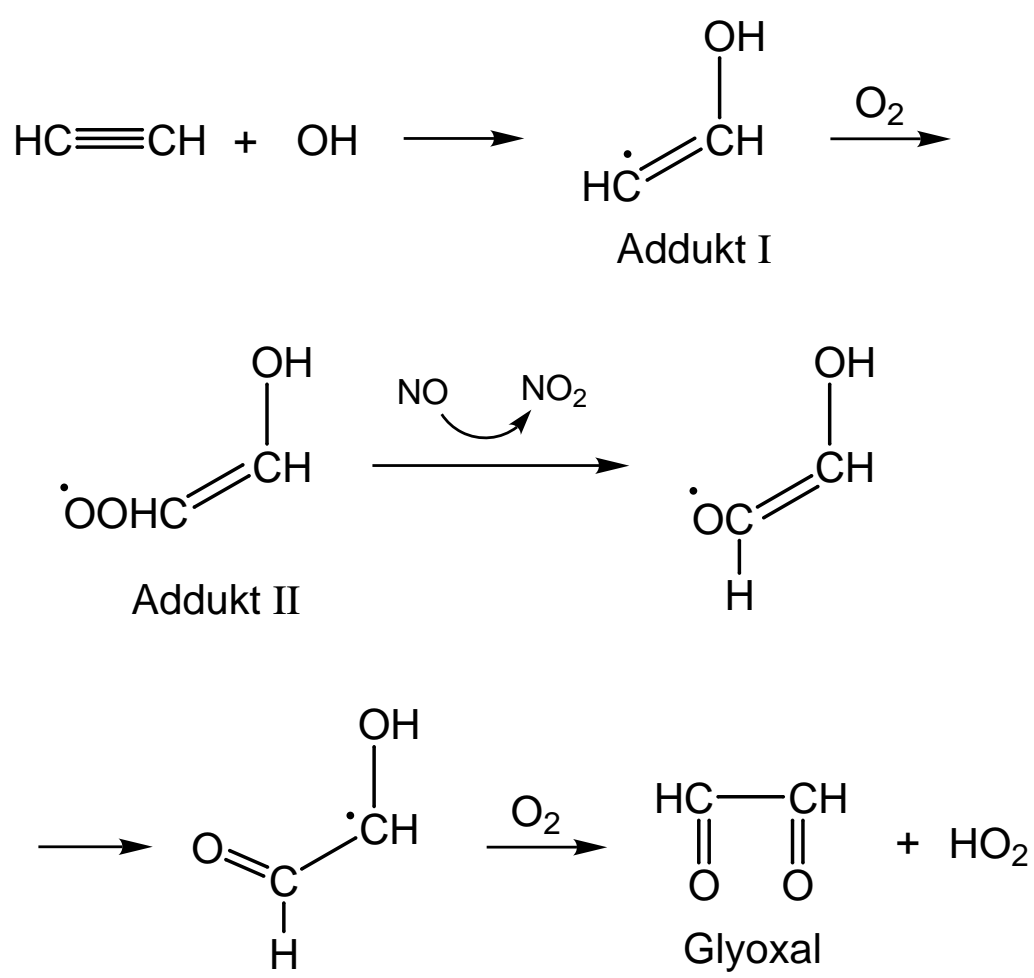

Abbildung 1.1: Reaktion 1.8 in Anwesenheit von $\mathrm{O}_{2}$

von Sørensen et al. bei ca. 10 bar eine starke Abweichung zu diesen Messungen zeigen. Eine Zusammenfassung der experimentell und theoretisch bestimmten Hochund Niederdruckgeschwindigkeitskonstanten findet sich in Ref. [68].

Weiterhin wurde das Verhalten von Reaktion 1.8 in Anwesenheit von Sauerstoff untersucht, um die Verbindung zur Atmosphärenchemie herzustellen [60,61,69,70,71]. Molekularer Sauerstoff spielt in diesem Fall eine bedeutende Rolle, indem er mit dem aus Reaktion 1.10 entstandenen Addukt I $\left(\mathrm{C}_{2} \mathrm{H}_{2} \mathrm{OH}\right)$ nach Abb. 1.1 zum entsprechenden Dicarbonyl (im Fall des Ethins zum Glyoxal $\left((\mathrm{CHO})_{2}\right)$ umgesetzt wird. Als weiteres Produkt wurde Ameisensäure beobachtet, welche durch die Umordnung und den Zerfall von Addukt II [61] gebildet werden kann. Siese et al. [69,70] untersuchten die Reaktion 1.9 mittels Blitzlichtphotolyse und anschließender Resonanzfluoreszenz. Sie beobachteten dabei einen biexponentiellen Abfall der $\mathrm{OH}-$ Konzentration, der auf zwei unterschiedliche Kanäle der Reaktion des Adduktes I mit $\mathrm{O}_{2}$ schließen lässt. Ein Kanal verläuft wie in Abb. 1.1 beschrieben, im anderen Fall werden direkt OH-Radikale und Glyoxal gebildet. Als weiteres Zwischenprodukt wurde das Vinoxyradikal [60] beobachtet, das durch die Isomerisierung des 


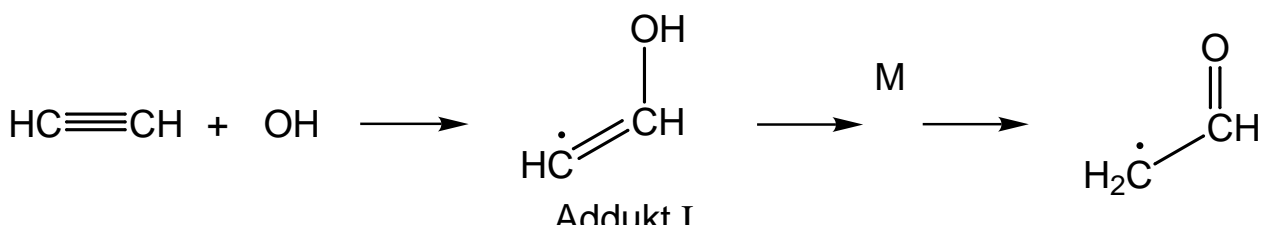

\section{Vinoxyradikal}

Abbildung 1.2: Isomerisierung zum Vinoxyradikal

ursprünglichen Adduktes I (Abb. 1.2) entsteht. Das entstandene Vinoxyradikal reagiert dann schnell mit $\mathrm{O}_{2}$ weiter zum Glyoxal.

Theoretische Untersuchungen zu Reaktion 1.8 wurden Mitte der 80er Jahre aufgrund der verbesserten Rechenleistung von Computern möglich. Die Berechnungen von Geschwindigkeitskonstanten reichen von einfachen Ansätzen wie der Theorie des Übergangszustandes (TST für Transition State Theory) bis hin zu Berechnungen nach der RRKM (Rice-Ramsperger-Kassel-Marcus)-Theorie [65, 64, 72, 63].

\section{$\mathrm{OH}+$ Propen bzw. Isopren}

Analog zu den Reaktionen des Ethens verlaufen die Reaktionen des Propens und Isoprens zunächst ebenfalls über den direkten Angriff des Hydroxylradikals an die Doppelbindung des Alkens. Für den Fall des Propens können sich bei der Addition von OH-Radikalen zwei Isomere bilden (vgl. Abb. 1.3). Greift das Hydroxylradikal das zentrale Kohlenstoffatom an, so wird ein primäres Radikal (1) gebildet. Bei einem Angriff an der terminalen Position entsteht ein sekundäres Radikal (2). Die entstehenden $\beta$-Hydroxypropenylradikale werden in der Atmosphäre mit Sauerstoff zu Peroxyradikalen umgesetzt [6], die wiederum in Anwesenheit von NO - ähnlich

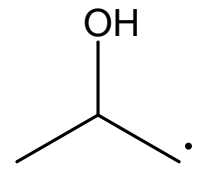

1

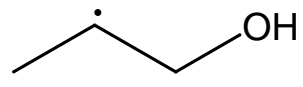

2

Abbildung 1.3: Bei der Reaktion des OH-Radikals mit Propen entstehende Isomere 


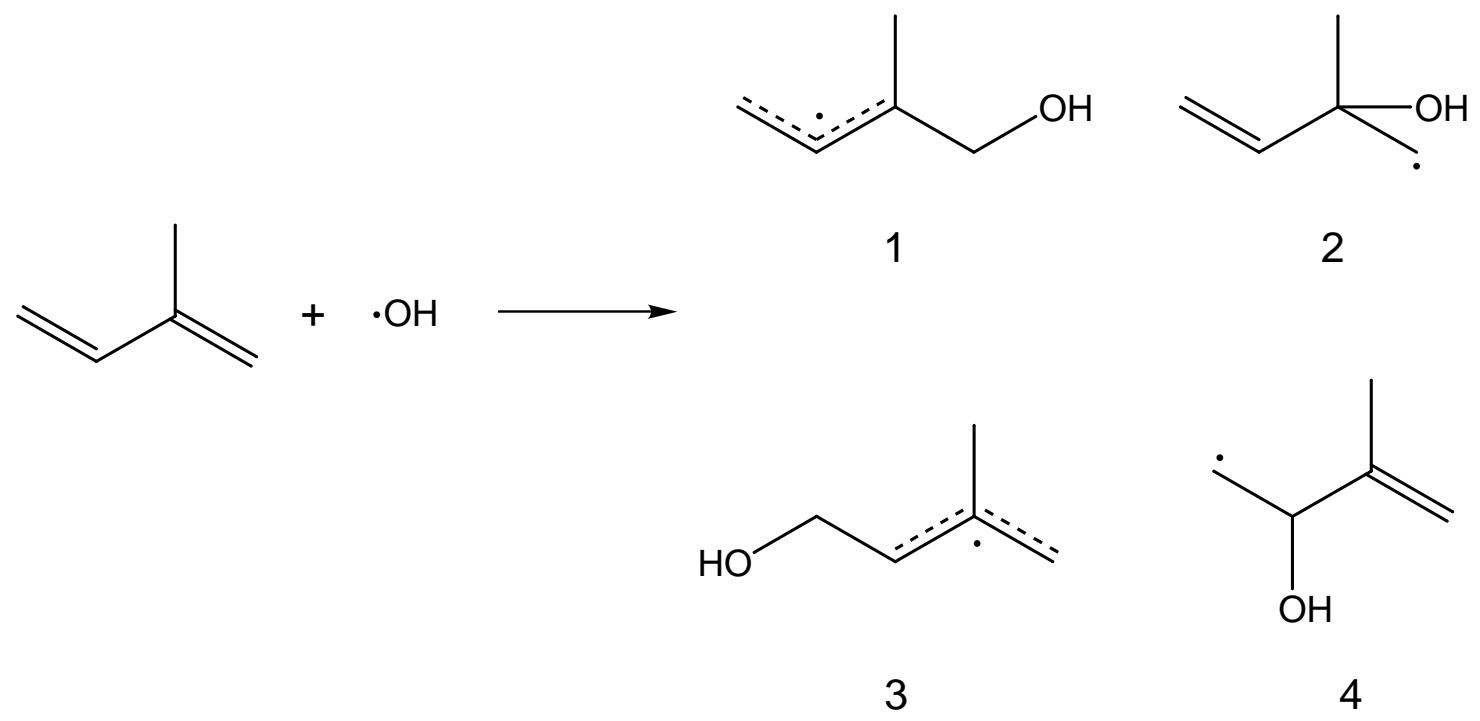

Abbildung 1.4: Bei der Reaktion des OH-Radikals mit Isopren entstehende Isomere

wie in Abb. 1.1 - zu Alkoxyradikalen weiterreagieren. Nach der Reaktion mit Sauerstoff zerfallen die Peroxyradikale in Formaldehyd, Acetaldehyd und $\mathrm{HO}_{2}$. Experimentelle Untersuchungen der Reaktion des OH-Radikals mit Propen beschränken sich mit einer Ausnahme auf die Bestimmung der Geschwindigkeitskonstanten bei Raumtemperatur [73,74,75, 28,76,77], einzig Vakhtin et al. [78] bestimmten einen Wert bei 103 K. Smith [79] führte ein temperaturabhängige Messung durch, die empfohlene Temperaturabhängigkeit ist in Ref. [76] angegeben. Theoretische Arbeiten liefern molekulare Parameter des OH-Propen-Adduktes wie Schwingungsfrequenzen und Energien [6, 80].

Die Addition des Hydroxylradikals an Isopren führt zu einer Reihe von Isomeren, von denen die vier stabilsten in Abb. 1.4 abgebildet sind. Die vier Isomere lassen sich in zwei Klassen einteilen. Wird das OH-Radikal an ein terminales Kohlenstoffatom addiert, so entstehen die Allylradikale 1 und 3, dabei findet eine Delokalisierung der Elektronen über drei Kohlenstoffatome statt. Bei einem Angriff auf eins der inneren Kohlenstoffatome werden die sekundären bzw. tertiären Radikale 2 und 4 gebildet. Die Weiterreaktion dieser Radikale in der Atmosphäre verläuft analog zur oben beschriebenen Reaktion der OH-Propen-Addukte. Je nach Struktur des bei der anfänglichen Addition gebildeten Radikals können als Endprodukte Methylvinylketon, Methacrolein, Formaldehyd und 3-Methylfuran entstehen [81] (vgl. Abb. 1.5). Die Bestimmung der Geschwindigkeitskonstanten der Reaktion von Hydroxylradikalen mit Isopren bei Raumtemperatur ist aufgrund der atmosphärenchemischen Relevanz zahlreich [23, 25, 82, 75, 83, 8, 84, 31, 85], wobei in Ref. [31] ebenfalls die 


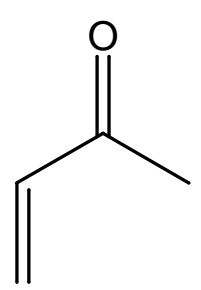

Methylvinylketon

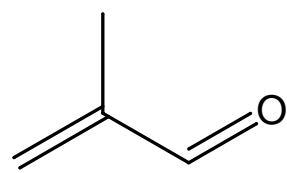

Methacrolein

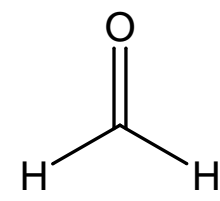

Formaldehyd

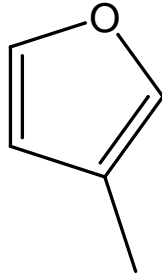

3-Methylfuran

Abbildung 1.5: Hauptprodukte der Oxidation von Isopren in der Atmosphäre

Druckabhängigkeit untersucht wurde. Temperaturabhängige Messungen oberhalb von $300 \mathrm{~K}$ finden sich in Ref. [86, 87], bei Ref. [80, 88, 89] handelt es sich um theoretische Arbeiten.

Weitere Details der bisherigen Untersuchungen der Reaktionen des Hydroxylradikals mit Propen und Isopren finden sich in Ref. [90].

\section{Interstellare Prozesse}

Darüberhinaus spielen Kohlenwasserstoffe außerhalb der Erdatmosphäre eine wichtige Rolle für die Zusammensetzung verschiedener Planeten und ihrer Satelliten. Trotz der Tatsache, dass z. B. die Atmosphären von Jupiter und Saturn vergleichsweise geringe Dichten haben, bestehen sie nicht nur aus leichten Elementen. Neben den Hauptkomponenten wie Wasserstoff, Helium, Methan und Ammoniak wurden in den Atmosphären von Jupiter und Saturn auch Ethin und Ethen gefunden. So kann z. B. Ethen durch einen photolytischen Prozess in Ethin überführt werden (Reaktion 1.12), welches aber photochemisch relativ stabil ist, da seine Dissoziationsprodukte $\left(\mathrm{C}_{2} \mathrm{H}\right.$ und $\left.\mathrm{C}_{2}\right)$ mit $\mathrm{H}_{2}$ wieder Ethin zurückbilden.

$$
\mathrm{C}_{2} \mathrm{H}_{4}+\mathrm{h} \nu \longrightarrow \mathrm{C}_{2} \mathrm{H}_{2}+\mathrm{H}_{2}
$$

Neben Sternen und Planeten, die ca. $90 \%$ der Masse unserer Galaxie enthalten, sind es sogenannte interstellare Wolken, die eine Anhäufung von Materie darstellen, während sich der Rest der Materie relativ gleichmäßig auf den Raum verteilt. Interstellare Wolken werden in dichte und diffuse eingeteilt. Teilchendichten variieren zwischen $10^{2} \mathrm{~cm}^{-3}$ (diffus) und $10^{6} \mathrm{~cm}^{-3}$ (dicht), die Temperaturen reichen von 10 bis $100 \mathrm{~K}$. In diesen Wolken wurden einige polyatomare Moleküle entdeckt, darunter auch Ethin sowie Cyanopolyethine $\left(\mathrm{H}(\mathrm{C} \equiv \mathrm{C})_{n} \mathrm{CN}\right)$ mit $n$ bis zu 10 . 
Sowohl Alkane, Alkene und auch Alkine haben somit eine wichtige Rolle in den Atmosphären diverser Planeten inne, insbesondere der Erde, wobei in diesem speziellen Fall vor allem der Abbau anthropogener Emissionen der oben genannte Spezies im Vordergrund steht. Für diesen Abbauprozess ist im wesentlichen das Hydroxylradikal verantwortlich, das trotz seiner geringen Konzentration zu den wichtigsten Oxidantien der Troposphäre gehört.

Das Studium der Reaktionen von Hydroxylradikalen mit ungesättigten Kohlenwasserstoffen gehört aufgrund der eben beschriebenen Relevanz für die Atmosphärenchemie zur aktuellen Forschung in der Physikalischen Chemie. Vor allem die Kenntnis der Geschwindigkeit solcher Reaktionen ist für Modelle, die die atmosphärischen Konzentrationen von Hydroxylradikalen und anderen Spezies beschreiben sollen, besonders wichtig. Dazu reicht es nicht aus, die Geschwindigkeitskonstanten bei Raumtemperatur experimentell zu bestimmen, da die Temperaturen in der Atmosphäre je nach Höhe variieren, sie erreichen ein Minimum von $215 \mathrm{~K}$ am unteren Ende der Stratosphäre und können lokal und unter gewissen Umständen bis auf $180 \mathrm{~K}$ absinken [91]. Auch die Zusammensetzung anderer Planeten ist von großem Interesse, vor allem sind aber die Reaktionen, die im interstellaren Raum ablaufen, kaum verstanden. In den interstellaren Wolken findet die Bildung von Molekülen unter sehr extremen Bedingungen statt [92,93,94], deshalb liegen in ihnen oft andere Reaktionsmechanismen als bei Raumtemperatur vor.

Aus diesem Grund ist es unbedingt nötig zu wissen, wie sich die Reaktionsmechanismen zu diesen tiefen Temperaturen hin verändern. Eine verlässliche Extrapolation von Geschwindigkeitskonstanten zu tiefen Temperaturen, die aus Messungen bei Raumtemperatur und darüber gewonnen wurden, kann nur dann gelingen, wenn kein Wechsel des Reaktionsmechanismus stattfindet. Zum Beispiel erscheint die Extrapolation temperaturabhängiger Daten aus Verbrennungsexperimenten zu atmosphärischen Temperaturen nur wenig sinnvoll. Umgekehrt ist es wichtig, Kenntnis über die Temperaturabhängigkeit von atmosphärenchemisch relevanten Reaktionen zu haben, um Aussagen über den Reaktionsmechanismus und damit über die Potentialfläche, auf der die Reaktion abläuft, treffen zu können.

\section{Reaktionen bei tiefen Temperaturen}

Die Temperaturabhängigkeit der Geschwindigkeitskonstanten $k(T)$ vieler Reaktionen gehorcht einer empirischen Formel, der Arrhenius-Gleichung [95]

$$
k(T)=A \cdot \exp \left(-E_{\mathrm{A}} / \mathrm{R} T\right) .
$$




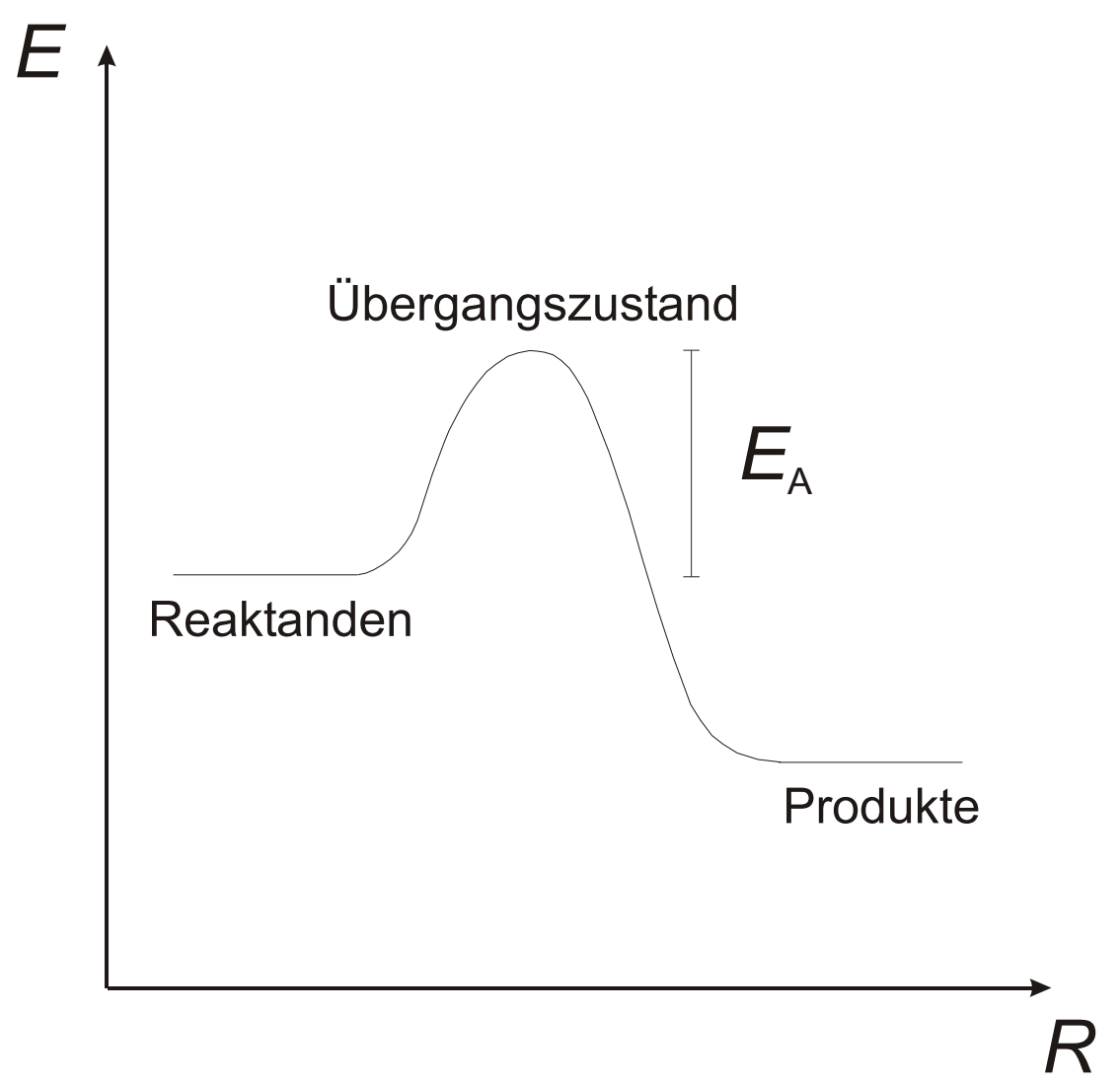

Abbildung 1.6: Schematischer Energieverlauf einer Reaktion mit Barriere

Dabei bezeichnet $A$ den sog. Arrhenius-Parameter. $E_{\mathrm{A}}$ ist die Aktivierungsenergie, die aufgewendet werden muss, um von den Reaktanden über den Übergangszustand zu den Produkten entlang der Reaktionskoordinate zu gelangen (vgl. Abb. 1.6). Der Nutzen der Arrhenius-Gleichung liegt darin, dass die Aktivierungsenergie aus einer geeigneten Auftragung (ln $k$ gegen 1/T) bestimmt werden kann. Voraussetzung für eine positive Temperaturabhängigkeit der Geschwindigkeitskonstanten nach Gl. 1.13 ist wiederum, dass eine genügend ausgeprägte Barriere zur Aktivierung vorhanden ist. Führt der Reaktionsweg aber über einen aus den Reaktanden gebildeten Stoßkomplex, dessen Bildung im Allgemeinen keine Aktivierungsenergie benötigt, so kann eine Abweichung vom Arrhenius-Verhalten erwartet werden (Abb. 1.7). Eine solche Abweichung zeigen Ion-Molekül-Reaktionen, die oft ein wie in Abb. 1.7 gezeigtes Energieprofil besitzen. Dabei spielen vor allem langreichweitige Anziehungskräfte zwischen dem Ion und permanentem oder induziertem Dipol des Moleküls eine Rolle. Dabei kann davon ausgegangen werden, dass diese Kräfte effektiver wir- 


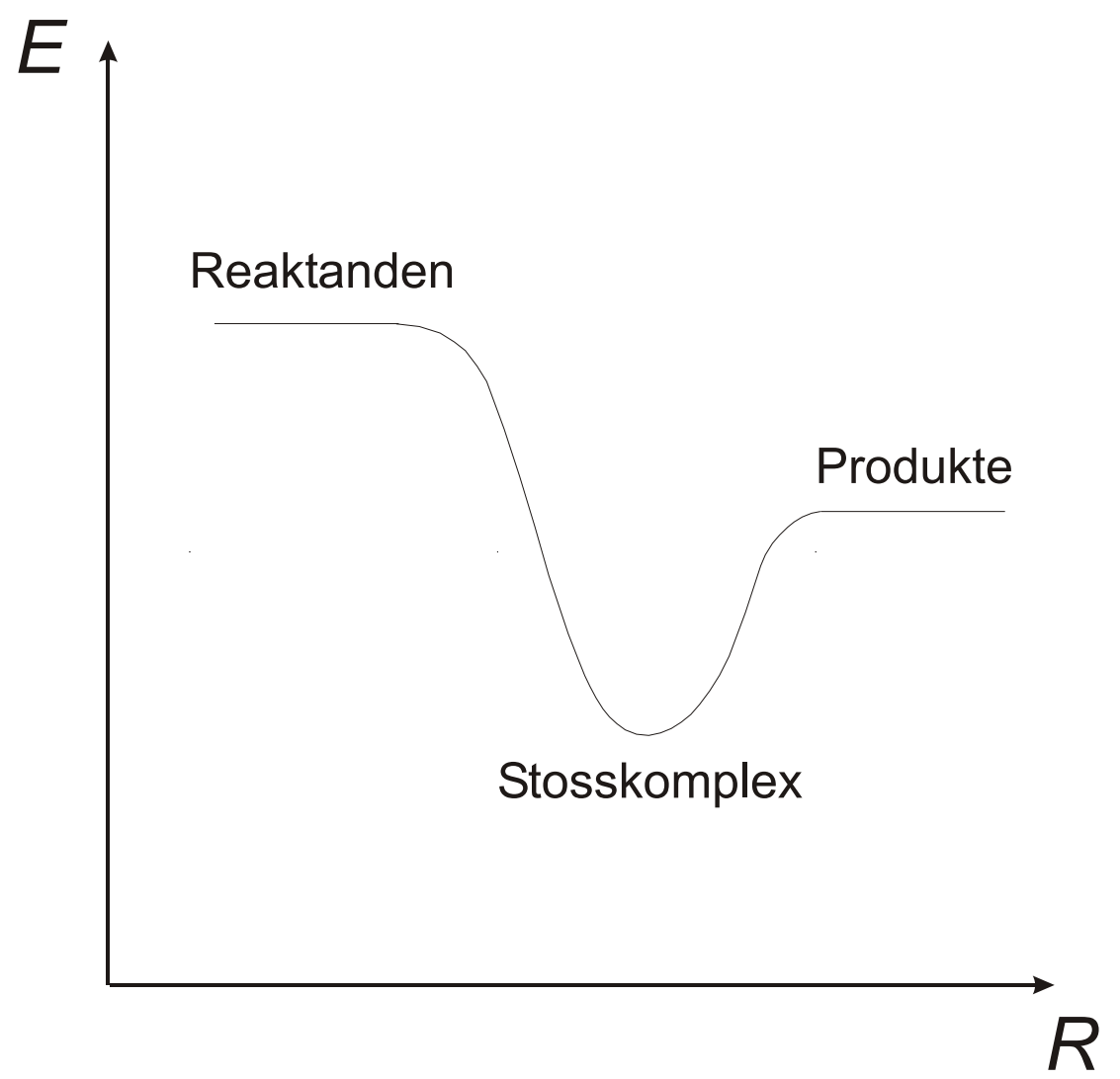

Abbildung 1.7: Schematischer Energieverlauf einer Reaktion ohne Barriere

ken können, je geringer sich die Reaktanden bewegen, d. h. je geringer die Temperatur wird. Deswegen wird bei Ion-Molekül-Reaktionen häufig eine negative Temperaturabhängigkeit oder zumindest eine gleichbleibende Geschwindigkeitskonstante beobachtet [96]. Jedoch wurden auch Reaktionen zwischen zwei Neutralteilchen, insbesondere solche zwischen Radikalen und ungesättigten Molekülen, gefunden, deren Verhalten bei tiefen Temperaturen auf Potentialeigenschaften wie in Abb. 1.7 hindeuten $[97,98,99]$.

Experimentelle kinetische Studien seit Anfang der 70er Jahre beschränkten sich zunächst jedoch auf Temperaturen über $200 \mathrm{~K}$ [100]. Dies hatte zwei Gründe, zum einen wurden für die Modellierung atmosphärenchemischer Prozesse keine Daten für noch tiefere Temperaturen benötigt, da die Temperatur in der Atmosphäre selten unter $200 \mathrm{~K}$ absinkt. Zum anderen waren auch experimentelle Grenzen gegeben. Die Untersuchung der Reaktionen erfolgte meist in durch flüssigen Stickstoff gekühlten Flusssystemen, doch durch die Kondensation der Reaktanden war ein natürliches Limit des beobachtbaren Temperaturbereiches gegeben. Wie oben beschrieben, be- 
stand und besteht reges Interesse an kinetischen Daten unterhalb von $200 \mathrm{~K}$, einerseits aufgrund des mangelnden Verständnisses der Prozesse, die im interstellaren Raum ablaufen, aber vor allem, weil die gemessenen Geschwindigkeitskonstanten in diesem Bereich sehr empfindlich auf Eigenschaften der Potentialfläche, auf der die Reaktion abläuft, reagieren. Die thermische Energie, die die Reaktanden bei diesen tiefen Temperaturen besitzen, ist so gering, dass sogar eine wenig ausgeprägte Barriere ausreicht, um die Reaktion zu erschweren, ja sogar zu verhindern. Bei Raumtemperatur oder darüber wirkt sich eine solche kleine Barriere kaum auf die Geschwindigkeit der Reaktion aus. Aus diesem Grund können experimentelle kinetische Untersuchungen erheblich zum Verständnis von Reaktionsmechanismen beitragen. Weiterhin sind experimentelle Daten immens wichtig, um die Vorhersagen theoretischer Modelle zu testen.

Die Vorhersage einer Geschwindigkeitskonstante durch ein theoretisches Modell bei einer Temperatur gelingt in den meisten Fällen, da viele Modelle mindestens einen anpassbaren Parameter haben. Ist eine Theorie aber in der Lage, zumindest einen gewissen Bereich der Temperaturabhängigkeit wiederzugeben, so kann die Theorie als erfolgreich gewertet werden. Auch die Überprüfung der Qualität von theoretisch ermittelten Potentialflächen durch experimentelle Tieftemperaturdaten kann sinnvoll sein, da das untersuchte System bei tiefen Temperaturen, also kleinen thermischen Energien, sehr empfindlich auf kleine Reaktionsbarrieren und andere Details der Potentialfläche im Bereich chemisch relevanter Energien reagiert. Bis Mitte der 80er Jahre gab es allerdings, mit einer Ausnahme (die Rekombination von Wasserstoffatomen bis $77 \mathrm{~K}[101,102])$, keine experimentellen kinetischen Daten unter 200 K. Mitte der 80er Jahre wurde von Rowe et al. eine Technik entwickelt, die es ermöglicht, kinetische Studien bis zu $10 \mathrm{~K}$ durchzuführen.

Die sogenannte CRESU-Technik (Cinétique de Réaction en Ecoulement Supersonic Uniforme) [103, 104], die später von einigen anderen Gruppen adaptiert wurde, nutzt einen uniformen Überschalldüsenstrahl, der sich durch einen in Temperatur, Druck und Dichte konstanten kalten Fluss auszeichnet, ohne dabei die Kondensation der Reaktanden zu ermöglichen. Denn es handelt sich hierbei nicht um einen gekühlten Flussreaktor, sondern um eine Gasexpansion im evakuierten Raum. Dabei findet die Expansion nicht, wie bei freien Überschallexpansionen, durch eine einfache kreisförmige Öffnung, sondern durch eine speziell geformte Lavaldüse statt. Eine tiefergehende Beschreibung der Überschallexpansionen, der Entwicklung der CRESU-Technik und der Berechnung von Lavaldüsen finden sich in den Abschnitten 3.1 und 3.2. Die CRESU-Technik stellt also die einzige verlässliche Methode dar, kinetische Studien bei extrem tiefen Temperaturen durchzuführen. 
Jedoch beinhalten kinetische Studien meist nicht nur die Temperatur-, sondern häufig auch die Druckabhängigkeit der Reaktionsgeschwindigkeit. Handelt es sich beispielsweise um eine Assoziationsreaktion, in der das Assoziat zur Stabilisierung einen Stoßpartner (Puffergas) benötigt, so ist die Geschwindigkeitskonstante der Produktbildung abhängig vom Druck bzw. der Teilchendichte des Stoßpartners (siehe Abschnitt 2.1). Dabei lassen sich zwei charakteristische Bereiche definieren: es existiert ein Niederdruckbereich, in dem die Geschwindigkeitskonstante linear mit der Teilchendichte des Stoßgases verknüpft ist und ein Hochdruckbereich, in dem die Geschwindigkeit der Reaktion unabhängig vom Druck ist. Der Übergang zwischen den beiden Bereichen wird Fall-Off-Bereich genannt. Wann die Erhöhung der Geschwindigkeitskonstanten mit dem Druck eine Sättigung erreicht hat, hängt neben der Temperatur von der Größe des Moleküls - also von der Anzahl der Atome im Molekül - ab. Da die Lebenssdauer des Assoziates von der Anzahl der Schwingungsmoden ( $\rightarrow$ Größe des Moleküls) abhängt, erreichen größere Moleküle schon bei kleineren Drücken den Hochdruckbereich. Da sich die Drücke in CRESU-Apparaturen aufgrund der Begrenzung durch die Strömungsdynamik nur in einem kleinen Bereich von ca. zwei Größenordnungen variieren lassen, wurden bisher hauptsächlich druckunabhängige Reaktionen untersucht. Für druckabhängige Reaktionen kleiner Moleküle (wie in dieser Arbeit Ethin $\left(\mathrm{C}_{2} \mathrm{H}_{2}\right)$ bzw. Ethen $\left(\mathrm{C}_{2} \mathrm{H}_{4}\right)$ mit OH-Radikalen) liegen die bestimmten Geschwindigkeitskonstanten jedoch bei den in CRESU-Apparaturen vorliegenden Drücken wahrscheinlich im Fall-Off-Bereich und sind deshalb schwer einzuordnen.

Jedoch ist es möglich, eine gute Abschätzung des Hochdruckgrenzwertes zu erhalten, wird die Relaxationsgeschwindigkeitskonstante der reaktiven Spezies (in dieser Arbeit das Hydroxylradikal (OH)) in Anwesenheit des Reaktionspartners bestimmt. Dabei wird davon ausgegangen, dass jeder Stoß, der zur Relaxation führt, auch die Bildung eines Assoziates im Hochdruckgrenzfall ermöglicht. Die Vergleichbarkeit der Prozesse der Schwingungsrelaxation und der Reaktion im Hochdruckgrenzfall beruht darauf, dass in beiden Fällen derselbe Stoßkomplex aus dem Radikal und dem Reaktionspartner gebildet wird, dessen Bildung der geschwindigkeitsbestimmende Schritt der Reaktion ist. Darüberhinaus wird die Bildungsgeschwindigkeit des Stoßkomplexes als unabhängig vom Schwingungszustand des Radikals angenommen. In vielen Reaktionen ist der Hochdruckgrenzwert experimentell schwer zugänglich, da bei hohen Drücken z. B. Diffusion einsetzt und somit ein Wechsel des Mechanismus stattfindet, bevor der Hochdruckgrenzwert überhaupt erreicht wird. Die Abschätzung der Hochdruckgeschwindigkeitskonstanten einer Assoziationsreaktion über die Relaxation des schwingungsangeregten Radikals wurde schon für die Systeme NO $+\mathrm{O}$ [105], $\mathrm{OH}+\mathrm{NO}$ [99], $\mathrm{CN}+\mathrm{NO}$ [106], $\mathrm{CH}+\mathrm{H}_{2} / \mathrm{D}_{2}$ [107] und $\mathrm{OH}+\mathrm{CO}$ [108] erfolgreich angewendet. 
Die vorliegende Arbeit ist wie folgt gegliedert. Zunächst werden verschiedene Konzepte zur Beschreibung unimolekularer Reaktionen (Abschnitt 2.1) und die dazu nötige quantenchemische Ermittlung von Molekülparametern (Abschnitt 2.2) vorgestellt, zum Abschluss des theoretischen Kapitels findet sich eine Einführung in die spektroskopischen Eigenschaften des Hydroxylradikals (Abschnitt 2.3). Im experimentellen Teil (Kapitel 3) finden sich ein allgemeiner Abschnitt über Überschallexpansionen, ein spezieller Teil über Lavaldüsen und die Beschreibung des experimentellen Aufbaus. In Kapitel 4 werden zunächst die experimentellen und theoretischen Ergebnisse vorgestellt und anschließend in Kapitel 5 im Vergleich mit bisherigen Arbeiten diskutiert. Nach einem Ausblick auf zukünftige Studien mit Hilfe der aufgebauten CRESU-Apparatur sind in Anhang A die berechneten optimierten Geometrien, Frequenzen und Energien der beteiligten Moleküle, in Anhang B die Details der SACM-Rechnungen aufgeführt. 



\section{Kapitel 2}

\section{Theoretische Grundlagen}

In diesem Kapitel wird zunächst auf die verschiedenen Konzepte zur Beschreibung unimolekularer Reaktionsdynamik eingegangen. Neuere und aufwendige Theorien sind auf spezifische Molekülparameter der beteiligten Moleküle angewiesen, die sich heutzutage mit modernen quantenchemischen Methoden berechnen lassen, die im zweiten Teil diese Kapitels vorgestellt werden. Im letzten Teil dieses Kapitels werden die Spektroskopie und die sich daraus ergebenden Eigenschaften der laserinduzierten Fluoreszenz des Hydroxylradikals erläutert.

\subsection{Unimolekulare Reaktionsdynamik}

Wichtige Reaktionen in der Atmosphärenchemie wie Dissoziationen oder Isomerisierungen gehören zur Klasse der unimolekularen Reaktionen. In den folgenden Abschnitten wird die Entwicklung von einfachen Ansätzen zur theoretischen Beschreibung dieser Reaktionen bis hin zu aufwendigen Modellen beschrieben. Dabei steht die Vorstellung der Konzepte - und nicht die Aufführung der gesamten Gleichungen, die ein Modell enthält - im Vordergrund.

\subsubsection{Frühe Theorien}

In einer unimolekularen Reaktionen reagiert ein Reaktandmolekül A zu den Produkten P (Gl. 2.1).

$$
\mathrm{A} \stackrel{k_{\text {uni }}}{\longrightarrow} \mathrm{P}
$$

Lindemann entwickelte ein Modell für solch unimolekulare Reaktionen [109], das vor allem die Druckabhängigkeit der Geschwindigkeitskonstanten beschreiben sollte. 
Dabei wird die unimolekulare Reaktion in mehrere Teilschritte unterteilt. Der erste Schritt beschreibt die Aktivierung eines Reaktandenmoleküls A durch Stöße mit Badgasmolekülen M

$$
\mathrm{A}+\mathrm{M} \stackrel{k_{1}}{\longrightarrow} \mathrm{A}^{*}+\mathrm{M} .
$$

Entweder wird das aktivierte Reaktandmolekül A* desaktiviert,

$$
\mathrm{A}^{*}+\mathrm{M} \stackrel{k_{-1}}{\longrightarrow} \mathrm{A}+\mathrm{M}
$$

oder es reagiert zu den Produkten

$$
\mathrm{A}^{*} \stackrel{k_{2}}{\longrightarrow} \mathrm{P} .
$$

$k_{1}$ und $k_{-1}$ sind die Geschwindigkeitskonstanten für die Aktivierung bzw. Desaktivierung, $k_{2}$ beschreibt die Produktbildung. Dieses Modell beruht auf der Annahme, dass die Zeitskalen, auf denen die Stoßaktivierung (2.2, 2.3) und die eigentliche Reaktion (2.4) ablaufen, deutlich voneinander getrennt sind. Im allgemeinen ist diese Annahme auch recht gut erfüllt, typische Zeitskalen für die Reaktionen 2.2 und 2.3 betragen $100 \mathrm{fs}$, unimolekulare Reaktionen laufen im Allgemeinen auf der Nanosekundenzeitskala ab. Die Kombination der Geschwindigkeits-Zeit-Gesetze der Gleichungen 2.2 bis 2.4 ergibt unter der Annahme der Quasistationarität für [A*] für die Geschwindigkeit der Produktbildung:

$$
\frac{\mathrm{d}[\mathrm{P}]}{\mathrm{d} t}=\frac{k_{1} k_{2}[\mathrm{M}]}{k_{-1}[\mathrm{M}]+k_{2}}[\mathrm{~A}]=k_{\text {uni }}[\mathrm{A}]
$$

Es lassen sich zwei Grenzwerte für die Konzentration und damit den Druck des Badgases feststellen. Bei niedrigen Drücken ist die Aktivierung (2.2) und die Desaktivierung (2.3) für die Geschwindigkeit der Produktbildung entscheidend, d.h. wenn das Reaktandenmolekül A erst einmal aktiviert ist, so reagiert es fast immer zu den Produkten. Bei hohen Drücken findet hingegen eine schnelle Aktivierung bzw. Desaktivierung der Moleküle statt, es stellt sich ein Vorgleichgewicht zwischen den Reaktionen 2.2 und 2.3 ein. Als Grenzwerte für die Druckabhängigkeit der Geschwindigkeitskonstanten ergeben sich ein druckabhängiger Niederdruckgrenzwert $k_{0}$

$$
\lim _{[\mathrm{M}] \rightarrow 0} k_{\text {uni }}=k_{0}=k_{1} \cdot[\mathrm{M}]
$$

und ein druckunabhängiger Hochdruckgrenzwert $k_{\infty}$

$$
\lim _{[\mathrm{M}] \rightarrow \infty} k_{\text {uni }}=k_{\infty}=\frac{k_{1} k_{2}}{k_{-1}} .
$$




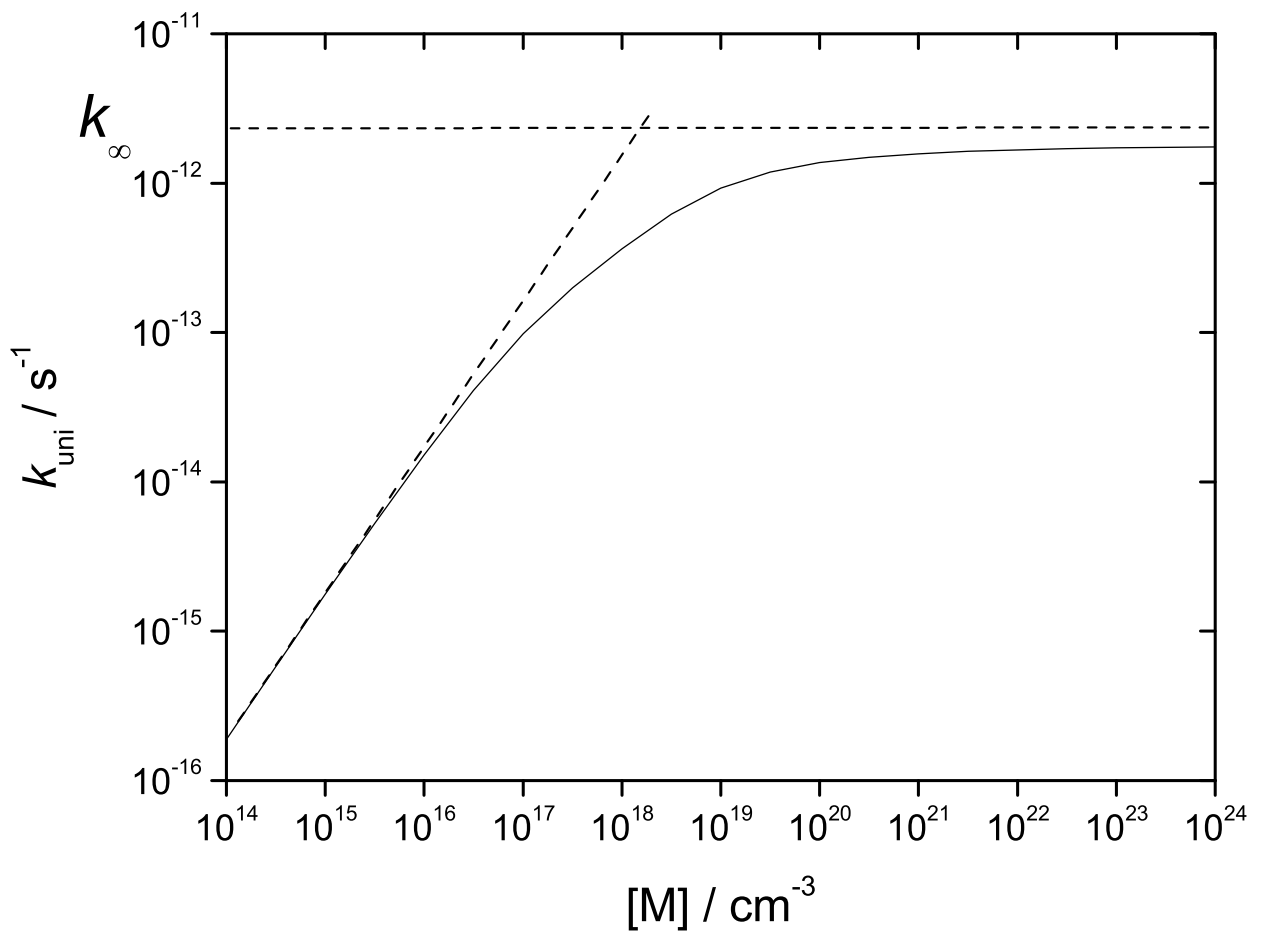

Abbildung 2.1: Fall-Off-Kurve

In einem Übergangsbereich zwischen diesen beiden Grenzbereichen, der Fall-OffBereich genannt wird, findet ein Wechsel der Reaktionsordnung von zweiter (Niederdruckbereich) nach erster Ordnung (Hochdruckbereich) statt (vgl. Abb.2.1). Da der Ansatz von Lindemann diesen Verlauf zwar qualitativ beschreiben kann, den FallOff-Bereich jedoch nicht quantitativ wiedergeben kann, verbesserte Hinshelwood das Modell [110], indem er die Energieabhängigkeit der Geschwindigkeitskonstanten des eigentlichen Reaktionsschrittes mit einbezog:

$$
\mathrm{A}^{*}(E) \stackrel{k(E)}{\longrightarrow} \mathrm{P}
$$

Somit wurde ein mikroskopisches Bild des Reaktionsschrittes erzeugt, welches in Abb. 2.2 gezeigt ist. Die mikroskopische Dynamik des Reaktionsschrittes (2.8) und die des Stoßenergietransfers (2.2 und 2.3) wurden daraufhin in Form einer MasterGleichung beschrieben (Gl. 2.9). In diesem Modell findet der Stoßenergietransfer zwischen Molekülen mit der Energie $E_{\mathrm{i}}$ und $E_{\mathrm{j}}$ mit einer Geschwindigkeitskonstan- 


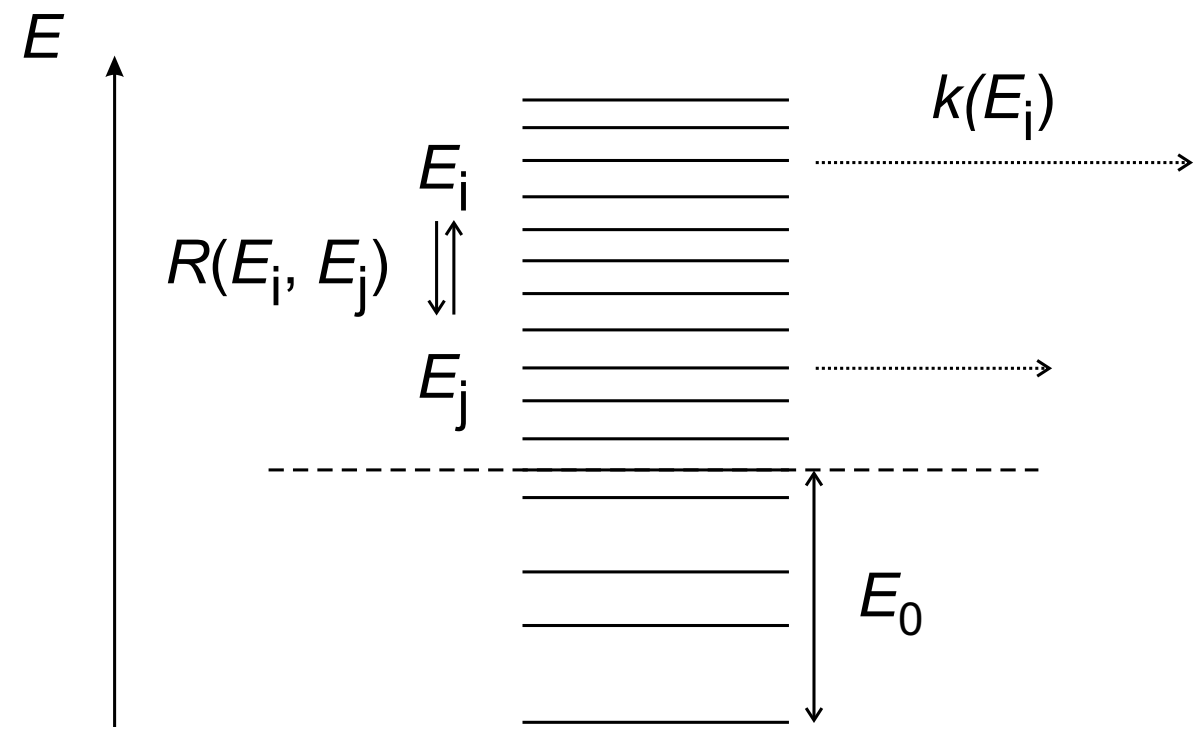

Abbildung 2.2: Mikroskopisches Bild einer unimolekularer Reaktion nach Lindemann-Hinshelwood

ten $R\left(E_{\mathrm{i}}, E_{\mathrm{j}}\right)$ statt, die Reaktion erfolgt von $E_{\mathrm{i}}$ mit $k\left(E_{\mathrm{i}}\right)$. Die zeitabhängige Population $g_{\mathrm{i}}$ des Zustandes i wird durch die Konkurrenz von Stößen und Reaktion bestimmt:

$$
\frac{\mathrm{d} g_{\mathrm{i}}}{\mathrm{d} t}=[\mathrm{M}] \sum_{\mathrm{i}}\left(g_{\mathrm{j}} R_{\mathrm{ij}}-g_{\mathrm{i}} R_{\mathrm{ji}}\right)-k_{\mathrm{i}} g_{\mathrm{i}}
$$

Angesichts der hohen Zustandsdichte kann die Summation durch eine Integration ersetzt werden, weiterhin kann die Energie- von der Zeitabhängigkeit separiert werden, die zeitabhängigen Terme fallen dann weg. $k_{\text {uni }}$ ergibt sich aus der zeitunabhängigen Master-Gleichung

$$
-k_{\text {uni }} g(E)=[\mathrm{M}] \int_{0}^{\infty}\left[R\left(E, E^{\prime}\right) g\left(E^{\prime}\right)-R\left(E^{\prime}, E\right) g(E)\right] \mathrm{d} E^{\prime}-k(E) g(E) .
$$

Der Verlauf von Fall-Off-Kurven läßst sich auch durch eine von Troe [111,112,113] entwickelte Formel beschreiben:

$$
k=\frac{k_{0}[\mathrm{M}]}{1+\left(k_{0}[\mathrm{M}] / k_{\infty}\right)} F_{\text {cent }}^{\left(1+\left[\log \left(k_{0}[\mathrm{M}] / k_{\infty}\right)\right]^{2}\right)^{-1}} .
$$

Dabei ist $F_{\text {cent }}$ der Stoßverbreiterungsfaktor, der die Differenz zwischen tatsächlichem (experimentellem) Fall-Off-Verhalten und dem nach dem Lindemann-Hinshel- 
wood vorhergesagten Verhalten korrigiert, da das Modell dazu neigt, die Geschwindigkeitskonstanten nahe der Mitte des Fall-Off-Bereiches zu überschätzen.

Polanyi [114] und Eyring [115] entwickelten dahingegen eine Theorie, die weniger die dynamischen Prozesse bei unimolekularen Reaktionen beschreibt (s. o.), sondern vielmehr auf rein statistischen Überlegungen beruht. In dieser Theorie des Übergangszustandes (TST für ,transition state theory“) werden dazu die Eigenschaften des Systems (Schwingungsfrequenzen, Rotationskonstanten usw.), insbesondere des Übergangszustandes (auch als aktivierter Komplex oder kritische Konfiguration bezeichnet) ermittelt. Die Geschwindigkeitskonstante für eine unimolekulare Reaktion wird nach der Theorie des Übergangszustandes als Geschwindigkeit berechnet, mit der sich das reagierende System durch den Übergangszustand (der dem Sattelpunkt auf der Reaktionskoordinate zwischen Reaktanden und Produkten darstellt) bewegt. Die Reaktionskoordinate entspricht in den meisten Fällen dem Pfad der minimalen Energie, der von Reaktanden zu Produkten führt. Wichtige Annahmen der TST sind:

- das reagierende System überquert den Übergangszustand nur einmal,

- der Übergangszustand hat einen Freiheitsgrad weniger als der Reaktand.

Der Ausdruck für die Hochdruckgeschwindigkeitskonstante nach der TST ergibt sich als

$$
k_{\mathrm{uni}}^{\infty}=\frac{k_{\mathrm{B}} T}{h} \frac{Q^{\ddagger}}{Q} \exp \left(\frac{-E_{0}}{k_{\mathrm{B}} T}\right) .
$$

$k_{\mathrm{B}}$ ist die Boltzmannkonstante, $h$ das Planck'sche Wirkungsquantum und $Q^{\ddagger}$ bzw. $Q$ sind die Zustandssummen des aktivierten Komplexes bzw. des Reaktanden. Zur Berechnung der Zustandssummen müssen molekulare Parameter der beteiligten Spezies (Schwingungsfrequenzen, Rotationskonstanten usw.) bekannt sein, die sich heutzutage mit theoretischen Methoden zuverlässig berechnen lassen. $E_{0}$ ist die Differenz der Nullpunktsenergien von aktiviertem Komplex und Reaktand. Eine Weiterentwicklung der eben vorgestellten Theorie ist die „variational transition state theory“, in der bei ungenauer Kenntnis des Übergangszustandes dessen Position optimiert wird, indem die Abhängigkeit der Geschwindigkeitskonstanten von der Lage des Übergangszustandes untersucht wird. Da die TST die Geschwindigkeitskonstanten unimolekularer Reaktionen im Allgemeinen überschätzt, wird nach dem Minimum von $k_{\text {uni }}$ gesucht. 


\subsubsection{Die RRKM-Theorie}

Die Theorie des Übergangszustandes (TST) liefert Geschwindigkeitskonstanten im Grenzfall hoher Drücke, in dem das thermodynamische Gleichgewicht zwischen aktivierten $\left(\mathrm{A}^{*}\right)$ und nicht-aktivierten Molekülen (A) eingestellt ist. Bei niedrigeren Drücken ist dies durch die geringere Stoßfrequenz nicht mehr gegeben, das System ist nicht in der Lage, die durch Reaktion verarmte Besetzung an aktivierten Molekülen wieder aufzufüllen. Dazu wurde eine Theorie entwickelt, die die TST auf ein mikrokanonisches Ensemble der angeregten Moleküle (abhängig von der Energie $E$ und dem Gesamtdrehimpuls $J$ ) anwendet. Diese Theorie wurde nach ihren Entwicklern Rice, Ramsperger [116], Kassel [117] und Marcus [118,119] als RRKM-Theorie bekannt. Wie in der TST wird angenommen, dass auf der Potentialfäche entlang der Reaktionskoordinate ein Punkt existiert, der, wenn er einmal überschritten ist, nicht wieder gekreuzt wird.

Weiterhin gilt das Prinzip der Ergodizität, das besagt, dass die Umverteilung der Energie, die die Moleküle zur Reaktion befähigt, innerhalb der Moleküle wesentlich schneller erfolgt als die eigentliche Reaktion. Außerdem bezeichnet $E$ in diesem Fall nicht die gesamte für die Reaktion zur Verfügung stehende Energie, sondern die Energie in den aktiven Freiheitsgraden (z. B. eine Schwingungsmode im Reaktanden). Als aktiv wird eine Freiheitsgrad bezeichnet, wenn er am Energieumverteilungsprozess teilnimmt. Damit die Reaktion stattfinden kann, muss genügend Energie in einer bestimmten Mode (die z. B. zum Bindungsbruch führt) lokalisiert sein. Die Geschwindigkeitskonstante für den Reaktionsschritt (Reaktion 2.8) nach der RRKM-Theorie lässt sich dann als Verhältnis der Anzahl der Zustände des aktivierten Komplexes $W^{\ddagger}(E)$ und der Zustandsdichte $\rho$ des Reaktanden ausdrücken:

$$
\begin{aligned}
k(E) & =\frac{W^{\ddagger}(E)}{h \rho(E)}, \\
W^{\ddagger}(E) & =\int_{0}^{E-E_{0}} \rho^{\ddagger}\left(E_{+}\right) \mathrm{d} E_{+},
\end{aligned}
$$

$E$ ist die Energie in den aktiven Moden (s. o.), $\rho^{\ddagger}\left(E_{+}\right)$ist die Zustandsdichte am Übergangszustand und $E_{0}$ die Schwellenenergie. Die Berechnung von Zustandsdichten $\rho(E)$ wird im folgenden Abschnitt erläutert. 


\subsubsection{Das SAC-Modell}

Die Modelle für die Berechnung von Geschwindigkeitskonstanten unimolekularer Reaktionen aus den vorhergehenden Abschnitten liefern für viele Reaktionen zufriedenstellende Ergebnisse. Voraussetzung für die Beschreibung einer Reaktion z. B. mit dem RRKM-Modell ist aber, dass die Position des Übergangszustandes auf dem Weg der minimalen Energie zwischen Edukten und Produkten genau bekannt ist. Darüberhinaus müssen seine Eigenschaften wie Rotationskonstanten, Schwingungsfrequenzen usw. berechnet bzw. abgeschätzt werden. Schwieriger wird die Berechnung, wenn die untersuchte Reaktion gar keine ausgeprägte Barriere und damit keinen Übergangszustand besitzt. Dies trifft z. B. für viele komplexbildende bimolekulare Reaktionen zu, in denen der Einfang der Reaktanden über langreichweitige Wechselwirkungen vollzogen wird und somit keine Aktivierungsenergie benötigt wird. Die Beschreibung solcher Reaktionen ist möglich, wenn die bimolekulare Reaktion als unimolekulare Rückreaktion des Adduktes zu den Edukten betrachtet wird. Dabei wird die Verknüpfung der Geschwindigkeitskonstanten für die Hin- $\left(k_{\mathrm{f}}\right)$ und Rückreaktion $\left(k_{\mathrm{r}}\right)$ über die Gleichgewichtskonstante $K_{\text {eq }}$, die aus den Zustandssummen $Q_{\mathrm{i}}$ berechnet werden kann, genutzt (Gl. 2.15 und 2.16).

$$
\begin{aligned}
\mathrm{A}+\mathrm{B} & \leftrightharpoons \mathrm{AB} \\
k_{\mathrm{f}} & =K_{\mathrm{eq}} k_{\mathrm{r}}=\frac{Q_{\mathrm{AB}}}{Q_{\mathrm{A}} Q_{\mathrm{B}}} \cdot k_{\mathrm{r}}
\end{aligned}
$$

Um das Problem der Lokalisierung des Übergangszustandes in Bindungsbruchreaktionen ohne adiabatische Barriere zu umgehen, entwickelten Troe und Quack das Modell der statistischen adiabatischen Reaktionskanäle (SACM für ,statisticical adiabatic channel model $\left.{ }^{6}\right)$ [120,121,122]. Zur Berechnung der Geschwindigkeitskonstanten wird eine ähnliche Gleichung wie in der RRKM-Theorie verwendet:

$$
k(E, J)=\frac{W(E, J)}{h \cdot \rho(E, J)} .
$$

$W(E, J)$ bezeichnet die Anzahl der offenen Reaktionskanäle, $h$ ist die Planck'sche Konstante und $\rho(E, J)$ die Zustandsdichte im betrachteten Energiebereich. Ein Kanal wird als offen bezeichnet, wenn seine maximale Energie unterhalb der Gesamtenergie $E$ liegt. Dabei ist es nicht von Belang, bei welchem Wert der Reaktionskoordinate das Energiemaximum erreicht wird. Verschiebt sich etwa das Maximum mit zunehmender Rotation, so ist eine Berechnung nach den Theorien aus den letzten Abschnitten erschwert. In Abb. 2.3 sind einige Potentialkurven abgebildet, dabei ist der einzige offene Kanal in diesem Fall Kanal 1, da sich seine Maximum unter der 


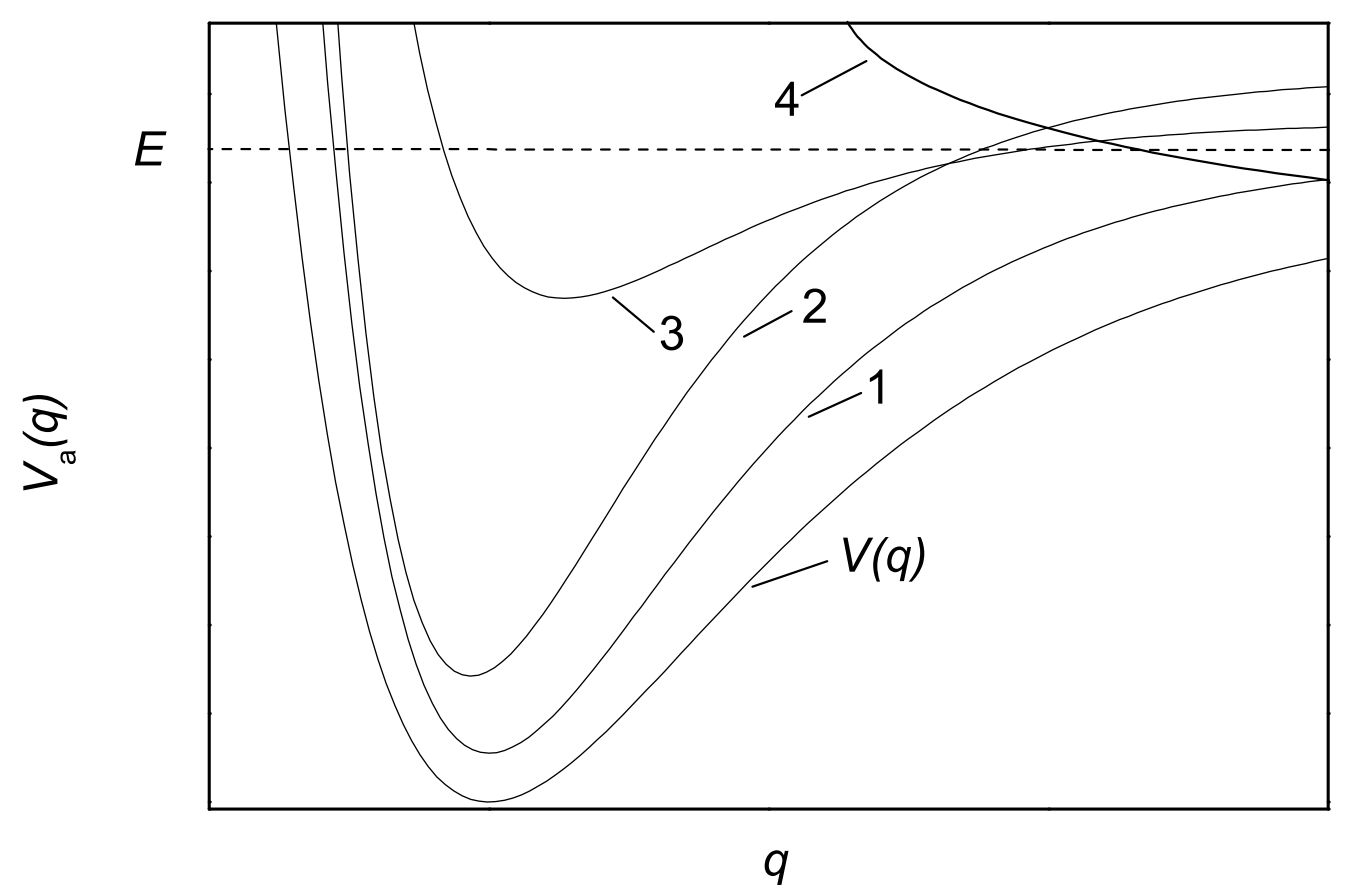

Abbildung 2.3: Adiabatische Potentialkurven

Gesamtenergie $E$ befindet. Im Fall des SAC-Modells wird ein Satz adiabatischer Potentialkurven konstruiert, d.h. für jeden einzelnen Quantenzustand wird abhängig vom Gesamtdrehimpuls $J$ die potentielle Energie entlang der Reaktionskoordinate berechnet. Die adiabatischen Kanalpotentiale $V_{\mathrm{a}}(q)$, die zur Bestimmung der Anzahl der offenen Kanäle $W(E, J)$ benötigt werden, werden als Summe aus einem Morsepotential $V(q)$ und den Kanaleigenwerten $E_{\mathrm{a}}(q)$ errechnet

$$
V_{\mathrm{a}}(q)=V(q)+E_{\mathrm{a}}(q) .
$$

Zur Berechnung der Kanaleigenwerte $E_{\mathrm{a}}(q)$ werden die Reaktandenzustände $\left|a_{n}\right\rangle$ mit den Zuständen des adiabatischen Kanals $\left|a_{q}\right\rangle$ und den Produktzuständen $\left|a_{p}\right\rangle$ korreliert. Bei dieser Korrelation bleiben gewisse Oszillatoren erhalten, einige verschwinden oder werden z. B. von einer Torsionsschwingung in die freie Rotation eines der Dissoziationsfragmente transformiert. Die Quantenzahlen der erhaltenen Oszillatoren bleiben gute Quantenzahlen, ebenso die den Drehimpuls betreffende Quantenzahl $J$, weiterhin ist die Energie eine Erhaltungsgröße. Die nicht erhaltenen Quantenzahlen (häufig Knickschwingungen betreffend und die Rotationsquanten- 
zahl) werden durch eine einzige Quantenzahl $w$ charakterisiert, ein Eduktoszillator wird als die Bewegung in der Reaktionskoordinate definiert. Weil die genaue Form des Potentials der Kanaleigenwerte jedoch nicht bekannt ist, wird eine einfache Interpolation zwischen Edukten $E_{\mathrm{a}}\left(q_{\mathrm{e}}, n\right)$ und Produkten $E_{\mathrm{a}}(q=\infty, p)$ anhand eines Interpolationsparameters $\alpha$ durchgeführt. Die Interpolation erfolgt durch eine Exponentialfunktion:

$$
\begin{aligned}
E_{\mathrm{a}}(q)= & E_{\mathrm{a}}\left(q_{\mathrm{e}}, n\right) \cdot \exp \left[-\alpha\left(q-q_{\mathrm{e}}\right)\right] \\
& +E_{\mathrm{a}}(q=\infty, p) \cdot\left(1-\exp \left[-\alpha\left(q-q_{\mathrm{e}}\right)\right]\right)+E_{\text {cent }} .
\end{aligned}
$$

$E_{\text {cent }}$ bezeichnet die Zentrifugalenergie und kann nach

$$
E_{\text {cent }}=B_{\mathrm{s}}(q) \cdot P(P+1)
$$

berechnet werden. $B_{\mathrm{s}}(q)$ ist eine Mittelung der Rotationskonstanten, $P$ ist eine pseudo-Rotationsquantenzahl und wird zwischen dem Gesamtdrehimpuls $J$ des Reaktanden und dem Drehimpuls $l$ der Fragmente umeinander interpoliert.

Die Berechnung der potentiellen Energie entlang der Reaktionskoordinate $V(q)$ wird durch ein Morsepotential beschrieben:

$$
\begin{aligned}
V(q) & =D\left\{1-\exp \left[-\beta\left(q-q_{\mathrm{e}}\right)\right]\right\}^{2} \quad \text { mit } \\
D & =\Delta H_{0}^{0}+E_{\mathrm{zn}}+E_{\mathrm{zp}}, \\
\beta & =\sqrt{\frac{F_{\mathrm{q}}}{2 D}}
\end{aligned}
$$

mit $D$ als Dissoziationsenergie, $\Delta H_{0}^{0}$ ist die Differenz der Energien von Reaktand und Fragmenten inklusive Nullpunktsenergien, $E_{\text {zn }}$ und $E_{\text {zp }}$ sind die Nullpunktsenergien des Reaktanden bzw. der Fragmente. Der Morseparameter $\beta$ kann nach Gl. 2.23 aus der Kraftkonstante $F_{\mathrm{q}}$ des Oszillators, der als Reaktionskoordinate bestimmt wurde, berechnet werden. In Abb. 2.4 sind wichtige Energien und ihr Zusammenhang schematisch dargestellt. Die Berechnung der Zustandsdichte $\rho(E)$ kann über verschiedene Verfahren erfolgen. Zunächst ist davon auszugehen, dass der Großteil der Freiheitsgrade eines polyatomaren Moleküls aus Schwingungen besteht; deswegen konzentriert sich die Berechnung von Zustandsdichten hauptsächlich auf die Schwingungsfreiheitsgrade. Klassisch lässt sich die Zustandsdichte aus der inversen Laplace-Transformation der Zustandssumme errechnen. Die klassische Zustandssumme $Q$ für ein System von $n$ harmonischen Oszillatoren mit den Frequenzen $\nu_{\mathrm{i}}$ ist gegeben als

$$
Q=\left(k_{\mathrm{B}} T\right)^{n} \prod_{\mathrm{i}=1}^{n}\left(\frac{1}{h \nu_{\mathrm{i}}}\right) .
$$




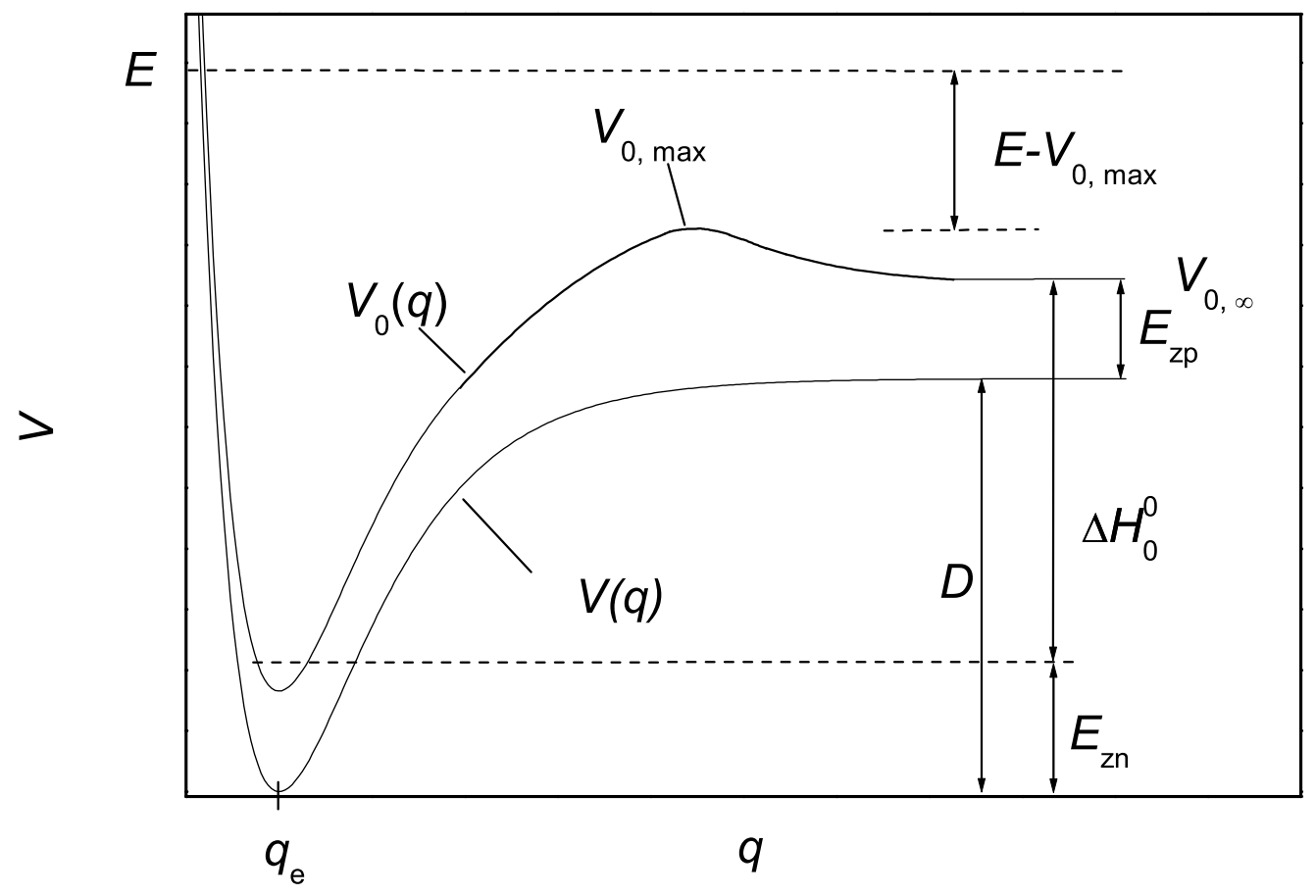

Abbildung 2.4: Potentialenergien und ihr Zusammenhang:

$$
V_{0}(q)=V(q)+\text { Nullpunktsenergien }
$$

Daraus ergibt sich durch inverse Laplace-Transformation die Zustandsdichte $\rho(E)$ :

$$
\rho(E)=\prod_{\mathrm{i}=1}^{n}\left(\frac{1}{h \nu_{\mathrm{i}}}\right) \frac{E^{n-1}}{(n-1) !} .
$$

In der klassischen Betrachtung des harmonischen Oszillators wird die Quantisierung völlig außer Acht gelassen, was die Aussagekraft der Berechnungen in Frage stellt. Whitten und Rabinovitch [123] führten eine Korrektur von Gl. 2.25 durch, indem sie die Nullpunktsenergie $E_{\mathrm{z}}$ mit einbezogen:

$$
\rho(E)=\prod_{\mathrm{i}=1}^{n}\left(\frac{1}{h \nu_{\mathrm{i}}}\right) \frac{\left(E+a E_{\mathrm{z}}\right)^{n-1}}{(n-1) !} .
$$

Dabei ist $a$ ein empirischer Parameter. Die exakte Berechnung der Schwingungszustandsdichte eines Moleküls ergibt sich aus allen möglichen Kombinationen der Schwingungsquantenzahlen, die in Einklang mit der Gesamtenergie zu bringen sind. 
Ein schneller Algorithmus zur exakten Berechnung der harmonischen Zustandsdichte wurde von Beyer und Swinehart [124] entwickelt. Dabei werden für jeden einzelnen Oszillator nacheinander die Zustände bis zur Gesamtenergie $E$ gezählt, wobei die Zustandsdichte iterativ modifiziert wird.

Somit ist eine Berechnung von Geschwindigkeitskonstanten nach dem SAC-Modell ein exzellentes Verfahren, um kinetische Informationen über Reaktionen zu erhalten, die ohne oder mit geringer Aktivierungsenergie ablaufen. Außerdem schließt das SAC-Modell die Lücke zwischen der RRKM-Theorie, die sich als sehr geeignet für Reaktionen mit starrem Übergangszustand bewährt hat, und der Phasenraumtheorie, welche vor allem für lockere Übergangszustände verwendet wird. Der Interpolationsparameter $\alpha$, der auch als Anisotropie- oder Paulingparameter bezeichnet wird, dient also auch als Maß für die Beschaffenheit des Übergangszustandes, d.h. ob er eher starr oder locker ist. Ein großer Wert für $\alpha$ steht dabei für einen eher lockeren Übergangszustand. Weiterhin ist das SAC-Modell in der Lage, inelastische Streuquerschnitte und Produktzustandsverteilungen zu berechnen [121]. Details und Ergebnisse der in dieser Arbeit durchgeführten Berechnungen nach dem SAC-Modell finden sich in Anhang B.

\subsubsection{Die Phasenraumtheorie (PST)}

Ein weiterer Ansatz zur Berechnung von Geschwindigkeitskonstanten unimolekularer Reaktionen ist die Phasenraumtheorie [125,126]. Der Phasenraum wird von Ortsvektor $\boldsymbol{q}$ und Impulsvektor $\boldsymbol{p}$ eines jeden Atoms eines Moleküls aufgespannt. Ist die Anzahl der Atome $N$, so ergibt sich ein $6 N$-dimensionaler Phasenraum. Damit ist jeder klassische Zustand des Moleküls exakt durch die Koordinaten im Phasenraum beschrieben. Da sich die Schwerpunktsbewegungen des Moleküls während der Reaktion nicht ändern, reduziert sich die Dimensionalität auf $6 N-6$. Werden die Bewegungsgleichungen für das System für einen Satz von Anfangsbedingungen gelöst, so ergibt sich eine zeitliche Entwicklung der Koordinaten im Phasenraum, die auch als Trajektorie bezeichnet wird. Nach einer Unterteilung des Phasenraums in eine Edukt- und eine Produktregion kann die Geschwindigkeitskonstante als Fluss der Trajektorien aus der Edukt- in die Produktregion angesehen werden. Anwendung findet die Phasenraumtheorie vor allem bei der Untersuchung von IonMolekül-Reaktionen, da sie davon ausgeht, das die internen Moden des Moleküls in der Übergangsregion (vergleichbar mit dem Übergangszustand) ähnlich denen der Produkte sind. Dies ist vor allem für Ion-Molekül-Reaktionen gegeben, da diese auf langreichweitigen Potentialen ablaufen, die Übergangsregion ist bei großen Ion-Molekül-Abständen zu finden. 
Gleichung 2.17 kann in verschieden Formen als Grundgleichung zur Berechnung von spezifischen Geschwindigkeitskonstanten $k(E, J)$ unimolekularer Reaktionen angesehen werden, die unterschiedlichen statistischen Modelle (RRKM, SACM, PST) unterscheiden sich lediglich in der Bestimmung der Anzahl der offenen Reaktionskanäle $W(E, J)$.

$$
k(E, J)=\frac{W(E, J)}{h \cdot \rho(E, J)}
$$

Sowohl die RRKM-Theorie als auch die Phasenraumtheorie lassen sich jeweils als Grenzfall des SAC-Modells auffassen. Für starre und vom Edukt aus gesehen frühe Übergangszustände eignet sich die RRKM-Theorie, die sich durch die SACMTheorie durch einen gegen null gehenden Paulingparameter $\alpha$ annähern lässt. Handelt es sich um eine Reaktion, die eine wenig ausgeprägte Barriere, also einen lockeren Übergangszustand besitzt, so kann die Phasenraumtheorie durch einen gegen eins strebenden Wert von $\alpha$ angenähert werden.

\subsection{Quantenchemische Bestimmung von Molekülparametern für die kinetische Modellierung}

Das in dieser Arbeit zur Berechnung von Geschwindigkeitskonstanten verwendete Modell der statistisch adiabatischen Kanäle (SACM) setzt voraus, dass die Eigenschaften des Reaktanden und der Produkte bekannt sind. Dazu gehören die Schwingungsfrequenzen, die Rotationskonstanten und einige andere Parameter aller beteiligten Spezies sowie die Dissoziationsenergie des Reaktanden. Denn zur Korrelation der Reaktand- und Produktoszillatoren müssen deren Schwingungsfrequenzen und Schwingungsformen (z. B. Knick- oder Streckschwingung) miteinander verglichen werden.

Zur Ermittlung der Molekülparameter können entweder spektroskopische Daten benutzt werden (soweit vorhanden), oder es kann ein Vergleich mit ähnlichen Molekülen, deren Eigenschaften gut bekannt sind, herangezogen werden. Dieser zweite Weg kann zu erheblichen Fehlern in der Berechnung der Geschwindigkeitskonstanten führen, falls ein sehr grober Vergleich der an der Reaktion beteiligten Spezies mit anderen Molekülen gezogen wird. Deswegen ist die Ermittlung von Molekülparametern nach modernen quantenchemischen Methoden eine exzellente Alternative zur Verwendung von experimentellen Werten, die z. B. für reaktive Intermediate (deren 
Zerfall berechnet werden soll) gar nicht vorhanden sind. Moderne quantenchemische Methoden können die Energie eines vorgegebenen Moleküls und verwandte Eigenschaften berechnen, mit Hilfe der Energieminimierung eine Optimierung der Molekülgeometrie vornehmen und Schwingungsfrequenzen berechnen. Dabei wird in den gängigsten Methoden die elektronische Struktur der Moleküle berechnet, deren Energieeigenwerte durch die Schrödingergleichung [127] gegeben sind (betrachtet wird im folgenden nur die zeitunabhängige Schrödingergleichung):

$$
\widehat{H} \psi=E \psi .
$$

Dabei ist $\widehat{H}$ der Hamiltonoperator, der sich aus einem Operator für die kinetische $(\widehat{T})$ und einem Operator für die potentielle Energie $(\widehat{V})$ zusammensetzt (Gl. 2.28). $\Psi$ ist die zeitunabhängige Wellenfunktion und $E$ steht für die Energieeigenwerte. Eine exakte Lösung der Schrödinger-Gleichung beschränkt sich auf Ein-ElektronenSysteme wie das Wasserstoffatom $(\mathrm{H})$ oder das Helium-Kation $\left(\mathrm{He}^{+}\right)$. Deshalb müssen für größere Atome oder Moleküle verschiedene Näherungsverfahren eingesetzt werden, die im Folgenden beschrieben werden. Dazu werden die Operatoren genauer betrachtet.

$$
\begin{aligned}
\widehat{H} & =\widehat{T}+\widehat{V} \\
\widehat{T} & =\widehat{T}_{\mathrm{K}}+\widehat{T}_{\mathrm{e}} \\
\widehat{V} & =\widehat{V}_{\mathrm{Ke}}+\widehat{V}_{\mathrm{KK}}+\widehat{V}_{\mathrm{ee}}
\end{aligned}
$$

Die mit K indizierten Größen beziehen sich auf die Atomkerne im Molekül, die mit e indizierten Größen auf die Elektronen. Somit steht der erste Operator in Gl. 2.30 für das attraktive Potential zwischen Kern und Elektron $\left(\widehat{V}_{\mathrm{Ke}}\right)$, der zweite und dritte Term für die repulsiven Kräfte zwischen den verschiedenen Kernen $\left(\widehat{V}_{\mathrm{KK}}\right)$ bzw. Elektronen $\left(\widehat{V}_{\text {ee }}\right)$. Da Atomkerne wesentlich schwerer sind als Elektronen, bewegen sie sich auch wesentlich langsamer. Deswegen kann in einer guten Näherung von einer Bewegung der Elektronen in einem Feld von festen Kernen gesprochen werden, d.h. die Bewegungen von Kernen und Elektronen sind separierbar. Diese als Born-Oppenheimer-Näherung [128] bekannte Feststellung hat den folgenden Effekt: da die Kerne sich quasi in Ruhe befinden, kann die kinetische Energie der Kerne vernachlässigt werden, außerdem ist die Kern-Kern-Abstoßung $\left(\widehat{V}_{\mathrm{KK}}\right)$ konstant. damit kann eine Art elektronische Schrödinger-Gleichung formuliert werden, die die Bewegung der Elektronen in einem Feld von festen Kernen beschreibt (elektronisches Problem):

$$
\widehat{H}_{\mathrm{el}} \psi_{\mathrm{el}}=E_{\mathrm{el}} \psi_{\mathrm{el}} .
$$


Wird nun Gl. 2.31 für verschiedene Kernkoordinaten gelöst, ergibt sich die Energie in Abhängigkeit der Kernkoordinaten. So wird eine Potential(hyper)fläche konstruiert. Analog zu obigem Vorgang kann nach der Born-Oppenheimer-Näherung die Bewegung der Kerne in einem Feld der Elektronen betrachtet werden, wobei für die Elektronenkoordinaten gemittelte Werte eingesetzt werden. Wird nun eine Kern-Schrödinger-Gleichung (analog zu Gl. 2.31) formuliert, so beschreibt sie die Bewegung der Kerne, denen die Elektronen instantan folgen, die in Schwingungen, Rotation und Translation der Moleküle unterteilt sind.

In dieser Arbeit wurden die optimierten Geometrien und damit Energien sowie die Frequenzen der beteiligten Moleküle bestimmt. Die Optimierung der Geometrie entspricht der Lokalisierung eines Minimums auf der Potentialfäche. Dazu wird die erste Ableitung der Energie nach den Ortskoordinaten gebildet. Nach der Eingabe einer zu optimierenden Geometrie wird die Energie und deren Gradient bestimmt, dessen Wert und das Vorzeichen die Richtung auf der Potentialfläche bestimmen, in der die Optimierung fortgeführt wird. Ist ein stationärer Punkt gefunden, d. h. der Gradient ist 0, so ist die Struktur optimiert. Zur Bestimmung der Frequenzen eines Moleküls wird die zweite Ableitung der Energie gebildet.

Die auf der Schrödinger-Gleichung basierenden Berechnungen verwenden im Gegensatz zu semiempirischen Methoden keine experimentellen Daten, sondern vielmehr einen minimalen Satz an physikalischen Konstanten: die Lichtgeschwindigkeit, die Masse und die Ladung von Elektron und Kern sowie die Planck'sche Konstante. Diese Methoden werden deshalb auch als ab initio-(von Grund auf) Methoden bezeichnet. Um quantenchemische Berechnungen von Molekülparametern vergleichen zu können, müssen die Methode - d. h. welche Art von Näherungen gemacht wurden, um die Schrödinger-Gleichung zu lösen - und der Basissatz angegeben werden. Ein Basissatz ist letztendlich die mathematische Beschreibung der elektronischen Wellenfunktion eines Systems, deren Quadrat als Aufenthaltswahrscheinlichkeitsdichte der Elektronen gedeutet werden kann. Damit sagt die Größe des Basissatzes etwas darüber aus, wieviel Raum den Elektronen zur Verfügung steht, um die optimale Konfiguration zu finden. Je größer der Basissatz gewählt wird, desto besser fällt die Näherung der Orbitale aus. Verschiedene Basissätze und ihre Bedeutung werden in Abschnitt 2.2.4 erläutert. Das Prinzip der in dieser Arbeit verwendeten Methoden wird in den folgenden Abschnitten (2.2.1, 2.2.2 und 2.2.3) jeweils kurz erläutert, Details finden sich in Ref. [129,130]. Dabei beschränken sich die Erläuterungen auf die Berechnung von Grundzustandsenergien. 


\subsubsection{Hartree-Fock-Methode (HF-SCF)}

Aufgrund der Tatsache, dass für die meisten Systeme eine exakte Lösung der Schrödingergleichung (2.27) nicht möglich ist, müssen Näherungsverfahren gefunden werden. $\mathrm{Zu}$ diesen gehört die Hartree-Fock-Methode. Sie beruht auf zwei Prinzipien, dem Variationsprinzip und der Methode der Selbstkonsistenz. Das Variationsprinzip beruht auf der Tatsache, dass jede beliebige Wellenfunktion $\phi$, die keine Eigenfunktion $\psi$ ist, einen Erwartungswert liefert, der immer größer ist als der exakte Eigenwert der Energie.

$$
\langle\phi|\widehat{H}| \phi\rangle>E
$$

Wird als sogenannte Testfunktion $\phi$ eine Funktion benutzt, die sich wie in der Molekülorbitaltheorie als Linearkombination von Wellenfunktionen darstellen lässt,

$$
\phi=\sum_{i=1}^{n} c_{\mathrm{i}} \phi_{\mathrm{i}}
$$

so lassen sich die Koeffizienten $c_{\mathrm{i}}$ anhand des Erwartungswertes optimieren. Der optimale Satz der Koeffizienten $c_{\mathrm{i}}$ wird also durch eine Minimierung des Erwartungswertes erreicht. Die Optimierung der Koeffizienten wird iterativ bis zur Selbstkonsistenz durchgeführt, d.h. die Energie ist letztendlich minimiert, nach der letzten durchgeführten Iteration tritt keine Veränderung der Koeffizienten mehr auf. Sie wird deswegen auch als HF-SCF- (für Hartree-Fock self consistent field) Methode abgekürzt [131, 132]. Die Hartree-Fock-Methode geht davon aus, dass jedes Elektron ein aus allen anderen Elektronen gemitteltes Feld spürt. Dieses Modell missachtet also die explizite Korrelation zwischen zwei einzelnen Elektronen, somit wird die sogenannte Austauschenergie zwischen Elektronen nicht berücksichtigt. Deshalb werden HF-Rechnungen oft als Startpunkt einer Berechnung mit Modellen, die die Elektronenkorrelation in verschiedener Weise berücksichtigen (post-SCFMethoden), benutzt.

\subsubsection{Störungstheorie $\left(\mathrm{MP}_{\mathrm{n}}\right)$}

Die Störungstheorie ist ein allgemeines Konzept, um den Einfluss einer Störung auf ein System mit Hilfe der Schrödinger-Gleichung zu beschreiben, und findet vielseitige Anwendungen. Zur näherungsweisen Lösung der Schrödinger-Gleichung wird ein Störoperator $\widehat{V}$ eingeführt:

$$
\widehat{H}=\widehat{H}_{0}+\lambda \widehat{V}
$$


Dabei ist $\widehat{H}$ der gestörte, $\widehat{H}_{0}$ der ungestörte Hamilton-Operator und $\lambda$ ein Parameter. Da die Störung $\lambda \widehat{H}$ als klein gegenüber $\widehat{H}_{0}$ angesehen wird, können die gestörte Wellenfunktion $\psi$ und die gestörte Energie $E$ mit dem Parameter $\lambda$ ausgedrückt werden:

$$
\begin{aligned}
& \psi=\psi^{(0)}+\lambda \psi^{(1)}+\lambda^{2} \psi^{(2)}+\lambda^{3} \psi^{(3)}+\ldots \\
& E=E^{(0)}+\lambda E^{(1)}+\lambda^{2} E^{(2)}+\lambda^{3} E^{(3)}+\ldots
\end{aligned}
$$

Werden $\psi$ und $E$ in dieser Form in die Schrödinger-Gleichung eingesetzt, ergibt sich folgende Gleichung:

$$
\left(\widehat{H}_{0}+\lambda \widehat{V}\right)\left(\psi^{(0)}+\lambda \psi^{(1)}+\ldots\right)=\left(E^{(0)}+\lambda E^{(1)}+\ldots\right)\left(\psi^{(0)}+\lambda \psi^{(1)}+\ldots\right)
$$

Daraus ergeben sich nach Ausmultiplizieren, Trennung nach den verschiedenen Potenzen von $\lambda$ und Umformen:

$$
\begin{aligned}
& \left(\widehat{H}_{0}-E^{(0)}\right) \psi^{(0)}=0 \\
& \left(\widehat{H}_{0}-E^{(0)}\right) \psi^{(1)}=\left(E^{(1)}-\widehat{V}\right) \psi^{(0)} \\
& \left(\widehat{H}_{0}-E^{(0)}\right) \psi^{(2)}=\left(E^{(1)}-\widehat{V}\right) \psi^{(1)}+E^{(2)} \psi^{(0)}
\end{aligned}
$$

Møller und Plesset, die in diesem Fall der Methode ihren Namen gaben, definierten den ungestörten Hamilton-Operator $\widehat{H}_{0}$ als Linearkombination von Einelektronenoperatoren $\widehat{F}_{\mathrm{i}}$, auch Fock-Operatoren genannt [133].

$$
\widehat{H}_{0}=\sum_{\mathrm{i}} \widehat{F}_{\mathrm{i}}
$$

Werden beide Seiten von Gleichung 2.38 mit $\left\langle\psi^{(0)}\right.$ multipliziert, so ergibt sich schlussendlich unter der Voraussetzung, dass es sich bei $\psi^{(n)}$ um orthonormale Funktionen handelt, $E^{(0)} . E^{(0)}$ ist nach der Definition von $\widehat{H}_{0}$ in Gl. 2.41 als Summe der Orbitalenergien $\epsilon_{\mathrm{i}}$ aufzufassen.

$$
\begin{aligned}
\left\langle\psi^{(0)}\left|\widehat{H}_{0}-E^{(0)}\right| \psi^{(0)}\right\rangle & =0 \\
\left.\Rightarrow \psi^{(0)}\left|\widehat{H}_{0}\right| \psi^{(0)}\right\rangle & =E^{(0)}\left\langle\psi^{(0)} \mid \psi^{(0)}\right\rangle=E^{(0)} \\
E^{(0)}=\left\langle\psi^{(0)}\left|\widehat{H}_{0}\right| \psi^{(0)}\right\rangle & =\sum_{\mathrm{i}} \epsilon_{\mathrm{i}}
\end{aligned}
$$


Analog kann mit Gl. 2.39 verfahren werden. Für $E^{(1)}$ und damit für die Summe von $E^{(0)}$ und $E^{(1)}$, die auch als Hartree-Fock-Energie bezeichnet wird, folgt:

$$
\begin{aligned}
E^{(1)} & =\left\langle\psi^{(0)}|\widehat{V}| \psi^{(0)}\right\rangle \\
E^{(0)}+E^{(1)} & =\left\langle\psi^{(0)}\left|\widehat{H}_{0}\right| \psi^{(0)}\right\rangle+\left\langle\psi^{(0)}|\widehat{V}| \psi^{(0)}\right\rangle \\
& =\left\langle\psi^{(0)}\left|\widehat{H}_{0}+\widehat{V}\right| \psi^{(0)}\right\rangle=\left\langle\psi^{(0)}|\widehat{H}| \psi^{(0)}\right\rangle=E^{\mathrm{HF}} .
\end{aligned}
$$

Nach der Bestimmung von $\psi^{(1)}$, der Störung der Wellenfunktion (1. Ordnung), kann dann der Wert von der $E^{(2)}$, der Störung der Hartree-Fock-Energie (1. Ordnung) bestimmt werden. Je nachdem, wie viele Glieder die Reihenentwicklung in Gl. 2.35 bzw. 2.36 aufgeführt sind, wird der Ansatz Störungstheorie 1., 2., 3. usw. Ordnung bezeichnet. MP2-Rechnungen liefern bei gleichen Basissätzen verbesserte Ergebnisse bezogen auf HF-Rechnungen, MP4-Rechnungen sind für kleinere Systeme praktikabel, erfordern jedoch erheblich höhere Rechenzeit.

\subsubsection{Dichtefunktionaltheorie (DFT)}

Die Dichtefunktionaltheorie unterscheidet sich von den beiden bereits vorgestellten Methoden dadurch, dass in diesem Fall nicht die Schrödinger-Gleichung gelöst werden muss, und dass eine Korrelation von Elektronen explizit berücksichtigt wird. Die Elektronen eines Systems bewegen sich nicht unabhängig voneinander, sondern reagieren auf die Bewegungen der anderen. Deswegen wird in der Dichtefunktionaltheorie die Elektronenkorrelation durch Funktionale der Elektronendichte berechnet. Ein Funktional ist mathematisch gesehen die Funktion einer Funktion. In der DFT werden Funktionale eingesetzt, die eine Funktion der Elektronendichte sind, die wiederum eine Funktion der Ortskoordinaten ist. Nach dem HohenbergKohn-Theorem existiert ein Funktional, das die Grundzustandsenergie $E$ und die Elektronendichte $\rho(r)$ exakt beschreiben kann [134]. Nach Kohn und Sham kann dieses Funktional angenähert werden, indem die elektronische Energie $E$ in mehrere Komponenten aufgeteilt wird [135]:

$$
\begin{aligned}
E & =E^{\mathrm{T}}+E^{\mathrm{V}}+E^{\mathrm{J}}+E^{\mathrm{XC}} \\
E^{\mathrm{XC}} & =E^{\mathrm{X}}+E^{\mathrm{C}}
\end{aligned}
$$

mit $E^{\mathrm{T}}$ als kinetischer Energie, $E^{\mathrm{V}}$ ist die potentielle Energie (Kern-ElektronAnziehung, Kern-Kern-Abstoßung), $E^{\mathrm{J}}$ ist die Coulomb-Energie (Elektron-ElektronAbstoßung) und $E^{\mathrm{XC}}$ wird als Austausch-Korrelations-Energie bezeichnet, die wiederum in Austausch- $\left(E^{\mathrm{X}}\right)$ und Korrelations-Komponenten $\left(E^{\mathrm{C}}\right)$ aufgeteilt wird. 
Dabei sind alle eben aufgeführten Energien (außer der Kern-Kern-Abstoßung) eine Funktion der Elektronendichte $\rho(r)$. Die verwendeten Funktionale unterscheiden sich dann hauptsächlich in der Art und Weise, wie Austausch- und KorrelationsKomponenten behandelt werden.

- Lokale Austausch- $\left(E_{\mathrm{LDA}}^{\mathrm{X}}\right)$ und Korrelations-Funktionale $\left(E_{\mathrm{LDA}}^{\mathrm{C}}\right)$ hängen nur von der Elektronendichte ab, beschreiben also ein uniformes Elektronengases und sind deswegen weitestgehend ungeeignet

- Gradientenkorrigierte (oder nicht-lokale) Funktionale enthalten sowohl die Abhängigkeit von der Elektronendichte als auch deren Gradienten. Die Kombination eines solchen Funktionals mit einem lokalen Funktional für die Austauschenergie wurde von Becke eingeführt [136, 137,138]. Ein gradientenkorrigiertes Korrelationsfunktional wurde von Lee, Yang und Parr entwickelt [139]. Eine Kombination aus diesen beiden wird dann als BLYP-Funktional bezeichnet.

- Hybridfunktionale sind Linearkombinationen von verschiedenen HartreeFock-, lokalen und gradientenkorrigierten Austauschfunktionalen. Die Korrelation kann entweder durch lokale und/oder gradientenkorrigierte Funktionale dargestellt werden.

$$
\begin{aligned}
E_{\mathrm{hybrid}}^{\mathrm{XC}}= & c_{\mathrm{HF}} E_{\mathrm{HF}}^{\mathrm{X}}+c_{\mathrm{DFT}} E_{\mathrm{DFT}}^{\mathrm{XC}} \\
E_{\mathrm{B} 3 \mathrm{LYP}}^{\mathrm{XC}}= & E_{\mathrm{LDA}}^{\mathrm{X}}+c_{0}\left(E_{\mathrm{HF}}^{\mathrm{X}}-E_{\mathrm{LDA}}^{\mathrm{X}}\right)+c_{\mathrm{X}} \Delta E_{\mathrm{B} 88}^{\mathrm{X}} \\
& +E_{\mathrm{VWN} 3}^{\mathrm{C}}+c_{\mathrm{C}}\left(E_{\mathrm{LYP}}^{\mathrm{C}}-E_{\mathrm{VWN} 3}^{\mathrm{C}}\right)
\end{aligned}
$$

Der Beitrag der einzelnen Funktionale in Gl. 2.50 lässt sich durch die einstellbaren Koeffizienten $c_{0}, c_{\mathrm{X}}$ und $c_{\mathrm{C}}$ regeln, die z. B. von Becke aus Atomisierungsenergien, Ionisierungspotentialen, Protonenaffinitäten und Atomenergien bestimmt wurden. $E_{\mathrm{VWN} 3}^{\mathrm{C}}$ ist das lokale Korrelationsfunktional nach Vosko, Wilk und Nusair [140]. Das in dieser Arbeit für die Rechnungen nach der Dichtefunktionaltheorie verwendete Funktional B3LYP wird also mit Hilfe von drei Parametern definiert. Die Berechnungen selber werden analog zur Hartree-Fock-Theorie bis zur Selbstkonsistenz durchgeführt.

\subsubsection{Basissätze}

Die mathematische Beschreibung von Atomorbitalen $\phi_{\mathrm{i}}$, deren Linearkombination die elektronische Wellenfunktion eines Systems annähert, erfolgt durch die Linearkombination von Basis-Funktionen $\chi_{\mu}$, dem Basissatz. Im Allgemeinen sind die 
Basis-Funktionen $\chi_{\mu}$ selbst Linearkombinationen von Gaußfunktionen $g_{\mathrm{p}}$, als Basisfunktionen werden aber auch einfache Gauß-Funktionen verwendet.

$$
\phi_{\mathrm{i}}=\sum_{\mu=1}^{N} \mathrm{c}_{\mu \mathrm{i}} \chi_{\mu} .
$$

Dabei sind $\mathrm{c}_{\mu \mathrm{i}}$ die Molekülorbitalausdehnungskoeffizienten.

$$
\chi_{\mu}=\sum_{p} \mathrm{~d}_{\mu \mathrm{p}} g_{\mathrm{p}}
$$

Die Bezeichnung von Basissätzen beruht auf der Anzahl der verwendeten Basisfunktionen und deren Eigenschaften. Für sog. minimale Basissätze werden atomähnliche Orbitale fester Größe verwendet, jedes Atom erhält den minimalen Satz an Orbitalen, z. B. für das Kohlenstoffatom die Orbitale 1s, $2 \mathrm{~s}, 2 \mathrm{p}_{\mathrm{x}}, 2 \mathrm{p}_{\mathrm{y}}, 2 \mathrm{p}_{\mathrm{z}}$. So besteht z. B. der STO-3G Basissatz aus drei einfachen Gaußfunktionen $(\rightarrow 3 \mathrm{G})$ pro Basisfunktion, STO steht für ,slater type orbitals“. Diese Slater-Orbitale sind zwar exakter bei der Berechnung von Molekülorbitalen, jedoch lässt sich eine Linearkombination von Gaußfunktionen schneller integrieren und spart somit wertvolle Rechenzeit. Eine Erweiterung des Basissatzes und somit eine Erhöhung der Genauigkeit der Berechnungen kann durch die Einführung von split-valence-Basissätzen erfolgen. Hier werden die Elektronen eines Moleküls in verschiedene Gruppen eingeteilt, die unterschiedlich großen Orbitalen zugeordnet werden. Dabei werden innere und Valenzelektronen unterschieden, die inneren Elektronen (auch Kernelektronen genannt) erhalten eine Basisfunktion. Je nachdem, ob die Valenzelektronen zwei oder drei verschiedene Basisfunktionen erhalten, werden die Basissätze als doublesplit- oder triple-split-Basissätze bezeichnet. Somit setzt sich der 6-31G-Basissatz aus 6 primitiven Gaußfunktionen für die Kernelektronen und drei bzw. eine für die Valenzelektronen zusammen. Die Einführung dieser auch als double-zeta- oder triple-zeta- bezeichneten Basissätze ermöglicht also die Veränderung der radialen Größe der Orbitale und wird damit den verschiedenen Atomgrößen gerecht.

Um auch die Form der Orbitale verändern zu können, wurden polarisierte Basissätze eingeführt. Dabei werden Basisfunktionen mit höheren Drehimpulsquantenzahlen (d-, p- oder f-Funktionen) zugesetzt, die Elektronen können sich auch weg von den Atomkernzentren bewegen, eine Polarisation der Moleküle ist möglich. Ein 6-31G(d,p)-Basissatz enthält also zusätzliche d-Funktionen für die Schweratome und p-Funktionen für Wasserstoff oder Heliumatome. Ein 6-31G(d)- bzw. 6-31G(d,p)-Basissatz wird auch als 6-31G*- bzw. 6-31G**-Basissatz bezeichnet. Die Vergrößerung des Basissatzes mit mehreren polarisierten Funktionen wird durch eine Ziffer vor den zugesetzten Funktionen angezeigt, wie in 6-31G(2d). Um den 
Orbitalen einen noch größeren Raum zu geben, können diffuse Funktionen eingeführt werden. Sie entsprechen übergroßen s- oder p-Funktionen und ermöglichen eine bessere Beschreibung von Systemen, in denen bestimmte Elektronen sehr weit vom Atomkernzentrum entfernt sind (Anionen, Moleküle mit freien Elektronenpaaren, angeregte Zustände usw.). Beziffert wird das Hinzufügen diffuser Funktionen für Schweratome mit einem „,+", für Wasserstoffatome mit einem „++", z. B. in $6-311++\mathrm{G}(3 \mathrm{df}, 3 \mathrm{pd})$.

Ein Vergleich der in dieser Arbeit mit verschiedenen Methoden und Basissätzen erzielten Ergebnisse findet sich in Anhang A, jedoch wurde keine systematische Variation der Methoden und Basissätze durchgeführt.

\subsection{Spektroskopie des Hydroxylradikals und laserinduzierte Fluoreszenz (LIF)}

Zur experimentellen Untersuchung der Geschwindigkeit einer chemischen Reaktion kann die zeitliche Entwicklung der Konzentration eines Reaktanden oder der Produkte herangezogen werden. In dieser Arbeit bietet sich dazu die Beobachtung des Hydroxylradikal über die laserinduzierte Fluoreszenz (LIF) an. Deswegen werden in den folgenden Abschnitten spektroskopische Eigenschaften, die für das Verständnis der Methode der LIF nötig sind, vorgestellt.

\subsubsection{Grundzustand $\left(X^{2} \Pi_{i}\right)$}

Der elektronische Grundzustand des Hydroxylradikals spaltet durch die starke SpinBahn-Kopplung in zwei Komponenten auf $\left(X^{2} \Pi_{1 / 2}\right.$ und $\left.X^{2} \Pi_{3 / 2}\right)$, jedes Rotationsniveau in einer Komponente spaltet wiederum in ein $\Lambda$-Dublett mit entgegengesetzter Parität auf $\left(X^{2} \Pi_{i}^{+}\right.$und $\left.X^{2} \Pi_{i}^{-}\right)$. Die Terme für die Rotationsenergie im Grundzustand werden mit $f_{1}\left(J=K+\frac{1}{2}\right)$ und $f_{2}\left(J=K-\frac{1}{2}\right)$ für die verschiedenen Spin-Bahn-Komponenten bezeichnet, die $\Lambda$-Dubletts mit $f_{1}^{\prime}$ bzw. $f_{2}^{\prime}$.

$$
\begin{aligned}
& f_{1}(K)=B\left[(K+1)^{2}-1-\frac{1}{2} \sqrt{4(K+1)^{2}+a(a-4)}\right]-D K^{2}(K+1)^{2} \\
& f_{2}(K)=B\left[K^{2}-1+\frac{1}{2} \sqrt{4\left(K^{2}+a(a-4)\right.}\right]-D K^{2}(K+1)^{2}
\end{aligned}
$$


Dabei sind in Gl. 2.53 und Gl. $2.54 B$ und $D$ die Rotationskonstanten, $K$ ist die Bahndrehimpulsquantenzahl und $a$ die sogenannte Kopplungskonstante.

\subsubsection{Erster angeregter Zustand $\left(A^{2} \Sigma_{\mathrm{i}}\right)$}

Auch der erste angeregte Zustand des Hydroxylradikals spaltet in zwei Komponenten auf:

$$
\begin{aligned}
& F_{1}(K)=B K(K+1)-D K^{2}(K+1)^{2}+R\left(K+\frac{1}{2}\right) \\
& F_{2}(K)=B K(K+1)-D K^{2}(K+1)^{2}-R\left(K+\frac{1}{2}\right)
\end{aligned}
$$

\subsubsection{Laserinduzierte Fluoreszenz}

Die Auswahlregeln für Übergänge zwischen den beiden Niveaus stellen sich folgendermaßen dar:

$$
\begin{array}{rll}
J \rightarrow J & \text { und } & J \pm 1 \rightarrow J, \\
K \rightarrow K & \text { und } & K \pm 1 \rightarrow K
\end{array}
$$

Daraus ergeben sich zwölf verschiedenen Zweige (2.59 bis 2.70), wobei die Zweige, die beide Auswahlregeln erfüllen, stark erscheinen. Wird die $K$-Auswahlregel verletzt, so werden schwache Banden erzeugt.

$$
\begin{array}{rlrl}
O_{12}(K) & =F_{1}(K-2)-f_{2}^{\prime}(K) & J-1 \rightarrow J \\
P_{1}(K) & =F_{1}(K-1)-f_{1}(K) & J-1 \rightarrow J \\
P_{2}(K) & =F_{2}(K-1)-f_{2}(K) & J-1 \rightarrow J \\
P_{12}(K) & =F_{1}(K-1)-f_{2}(K) & J & \rightarrow J \\
Q_{1}(K) & =F_{1}(K)-f_{1}^{\prime}(K) & J & \rightarrow J \\
Q_{21}(K) & =F_{2}(K)-f_{1}^{\prime}(K) & J-1 \rightarrow J \\
Q_{2}(K) & =F_{2}(K)-f_{2}^{\prime}(K) & J & \rightarrow J \\
Q_{12}(K) & =F_{1}(K)-f_{2}^{\prime}(K) & J+1 & \rightarrow J \\
R_{1}(K) & =F_{1}(K+1)-f_{1}(K) & J+1 & \rightarrow J \\
R_{21}(K) & =F_{2}(K+1)-f_{1}(K) & J & \rightarrow J \\
R_{2}(K) & =F_{2}(K+1)-f_{2}(K) & J+1 & \rightarrow J \\
S_{21}(K) & =F_{2}(K+2)-f_{1}^{\prime}(K) & J+1 & \rightarrow J
\end{array}
$$





\section{Kapitel 3}

\section{Experimenteller Teil}

\section{1 Überschallexpansion}

Die Überschallexpansion eines Gases ist eine weit verbreitete Methode, um eine Komprimierung der thermischen Besetzungsverteilung auf tiefe Energieniveaus der zu untersuchenden Moleküle zu erreichen. Diese Abkühlung ist z. B. von Nutzen für die Rotationsspektroskopie, da die Komplexität der Spektren abnimmt und so eine Bestimmung von Molekülparametern exakter oder überhaupt erfolgen kann. Dabei ist die gebräuchlichste Variante die freie Expansion.

Die freie Expansion wird experimentell durch das Ausströmen eines Gases aus einem Hochdruckreservoir durch eine kleine Öffnung in ein Niederdruckreservoir, idealer Weise ein Vakuum, realisiert. Dabei wird die Brown'sche Molekularbewegung im Hochdruckreservoir in eine gerichtete Bewegung im Niederdruckteil umgewandelt. Es bildet sich ein Gasstrahl aus, dessen Temperatur und Teilchendichte mit größer werdendem Abstand von der Öffnung (bis zu einem Abstand von ca. 20 Öffnungsdurchmessern) absinkt. Da in einem gewissen Abstand praktisch keine Stöße mehr zwischen den Molekülen stattfinden, herrscht im Gasstrahl kein thermisches Gleichgewicht. Dadurch ergibt sich eine enge Translationsgeschwindigkeitsverteilung der Moleküle. Für die Anwendung der freien Expansion z. B. in der Spektroskopie zur Entschlüsselung von bei Raumtemperatur komplexen Spektren sind dies optimale Voraussetzungen. Aufgrund des Temperaturgradienten sind die Anwendungen der freien Expansion für kinetische Studien jedoch begrenzt. Außerdem ist die Abkühlung in freien Expansionen auf einen Bereich zwischen 20 und $1 \mathrm{~K}$, die Teilchenzahldichte auf unter $10^{15} \mathrm{~cm}^{-3}$ limitiert [141].

Zur Präparation eines kalten Strahles, der in Dichte, Temperatur und Machzahl über mehrere Öffnungsdurchmeser konstant ist (ohne Gradienten), müssen Stöße zwischen Molekülen und Stöße von Molekülen mit einer Wand ermöglicht werden 


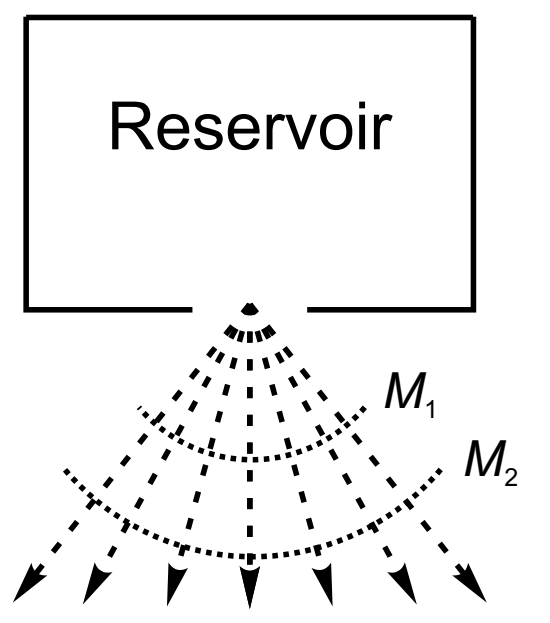

\section{freie Expansion \\ $\left(M_{2}<M_{1}\right)$}

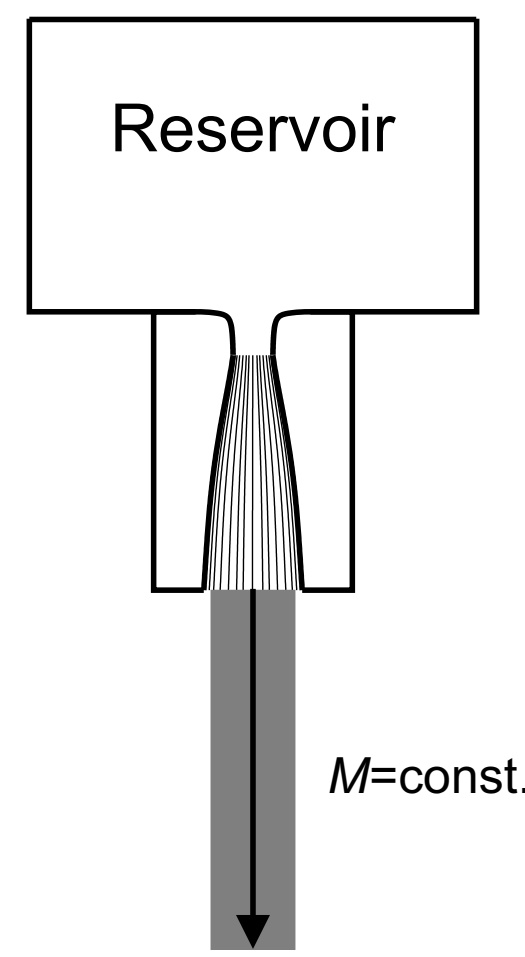

\section{Laval-Düsenexpansion}

Abbildung 3.1: Expansionsarten; $M$ : Machzahl (Gl. 3.1)

(vgl. Abb. 3.2). Das Prinzip der Lavaldüsenexpansion ist es daher, den Molekülen die Beschleunigung auf eine gewisse konstante Geschwindigkeit zu erlauben, es wird ein kaltes thermisches Ensemble von Molekülen erzeugt. Der isentrope Fluss wird anstelle einer punktförmigen Öffnung (freie Expansion) durch eine spezielle konvergent-divergent geformte Düse ermöglicht (vgl. Abb. 3.2). Der Bereich der präparierbaren thermischen Ensembles gestaltet sich bei Lavaldüsenexpansionen wesentlich breiter als bei freien Expansionen, die Temperatur kann von ca. 10 bis $300 \mathrm{~K}$, die Teilchenzahldichte zwischen $10^{16} \mathrm{~cm}^{-3}$ und $10^{18} \mathrm{~cm}^{-3}$ variiert werden. Es ist weiterhin möglich, Flüsse von mehr als 10 Düsenöffnungsdurchmessern Länge zu produzieren. Die grundsätzlichen Unterschiede zwischen freier und Lavaldüsenexpansion sind in Abb. 3.1 schematisch gezeigt.

Erfinder und Namensgeber der Lavaldüsen war der schwedische Wissenschaftler Gustaf de Laval. Der Sohn französischer Einwanderer (geboren 09.05.1845 in Osra, Schweden) studierte an der technischen Universität Stockholm und erlangte seinen 


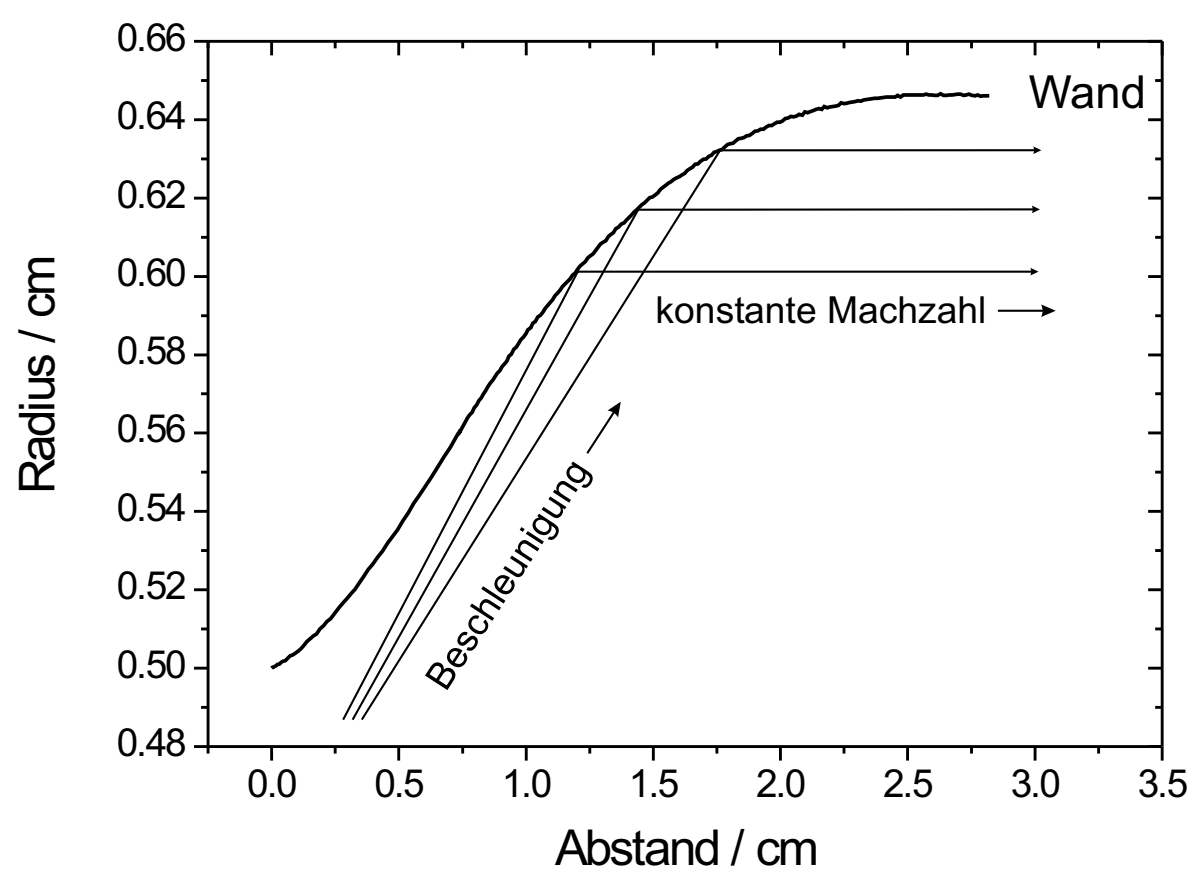

Abbildung 3.2: Prinzip der Lavaldüsenexpansion

Abschluss sowie den Doktorgrad in Ingenieurswissenschaften in Uppsala. Gustaf de Laval hatte vielfältige technische Talente, er produzierte 92 Patente und gründete 37 Firmen. 1878 erfand er einen Hochgeschwindigkeits-Zentrifugal-Abscheider für Milchrahm, der Anwendungen in vielen großen Molkereien auf der ganzen Welt fanden. Für die Turbine, die sich in dem Abscheider befand, entwickelte er eine „konvergent-divergente“-Düse, die später seinen Namen tragen sollte. Gustaf de Laval starb am 02.02.1913, sein Grabstein trägt die Inschrift: „The Man of High Speeds".

Anwendungen finden Lavaldüsen in Raketentriebwerken und vor allem in Windkanälen. Im Gegensatz zum Einsatz von Lavaldüsen in Windkanälen, in denen es vor allem auf eine möglichst isentrope Querschnittsfläche des Gasstrahls ankommt, ist ihre Anwendung für kinetische Untersuchungen bei tiefen Temperaturen hauptsächlich auf einen über eine längere Distanz konstanten Gasstrahl ausgerichtet. Diese Nutzung der Lavaldüsen erkannten Rowe et al. Mitte der achtziger Jahre und entwickelten in Rennes die sogenannte CRESU-Technik [103,104,142]. CRESU steht für Cinétique de Réaction en Ecoulement Supersonic Uniforme, also Reaktionski- 
netik in einem uniformen Überschalldüsenstrahl. Dabei untersuchten Rowe et al. Ion-Molekül-Reaktionen bis zu $8 \mathrm{~K}$, da diese bei den tiefen Temperaturen noch schnell genug sind, um gemessen zu werden. Ionen wurden mittels eines Elektronenstrahls erzeugt und mit einem Massenspektrometer detektiert. Anfang der neunziger Jahre adaptierte die Arbeitsgruppe von Sims und I. W. M. Smith die CRESUTechnik und etablierten eine CRESU-Apparatur in Birmingham zur Untersuchung von Reaktionen zweier Neutralteilchen bei tiefen Temperaturen. Die Beobachtung des Abbaus eines Reaktanden erfolgte über die Fluoreszenz, so dass vor allem die Reaktionen radikalischer Spezies wie CN- [143, 144, 145], OH- [146, 147, 148], CH$[149,150,151,107,152,153], \mathrm{C}_{2} \mathrm{H}$ - [154] und $\mathrm{CH}_{2}$-Radikalen [145] untersucht wurden. Kürzlich gelang es auch, Reaktionen des Kohlenstoffatoms bei Temperaturen zwischen 15 und $295 \mathrm{~K}$ zu beobachten $[155,156,157]$.

Die CRESU-Apparaturen in Rennes und Birmingham werden mit einem kontinuierlichen Gasfluss betrieben, deswegen ist eine hohe Pumpkapazität nötig, um das Vakuum aufrecht zu erhalten. Um die Pumpkapazität zu verringern, ist es sinnvoll die Expansion und damit das ganze Experiment gepulst zu betreiben, die benötigte Pumpkapazität wird erheblich reduziert. M. Smith entwickelte in Arizona eine gepulste Lavaldüsenapparatur, die Tieftemperaturdaten für einige Reaktionen des Hydroxylradikals lieferte $[158,159,160,161]$. Eine weitere gepulste Lavaldüsenapparatur wurde kürzlich in der Arbeitsgruppe von Leone aufgebaut, untersuchte Reaktionen beinhalten OH- [162] und $\mathrm{C}_{2} \mathrm{H}$-Radikale $[163,164,78,165]$, die zeitweise auch mit der Kopplung des Düsenstrahls an ein Massenspektrometer detektiert wurden. Als Reaktionspartner der oben angegebenen Radikale dienten neben Kohlenmonoxid $(\mathrm{CO})$, Sauerstoff $\left(\mathrm{O}_{2}\right)$ und Ammoniak $\left(\mathrm{NH}_{3}\right)$ und einigen anderen vor allem ungesättigte Kohlenwasserstoffe, davon war die Mehrheit verschiedene Alkene. Kürzlich konnte die Assoziation von Benzol zu Dimeren in einer CRESU-Apparatur untersucht werden [166]. Die im Rahmen dieser Arbeit in Göttingen aufgebaute gepulste CRESU-Apparatur lieferte bereits erste Ergebnisse zu Reaktionen des OH-Radikals mit ungesättigten Kohlenwasserstoffen bei tiefen Temperaturen [167, 90].

Außerhalb der Anwendung für die Reaktionskinetik werden die CRESU-Apparaturen auch zur Untersuchung von Relaxationsprozessen [168, 169,170], die Charakterisierung von Reaktionsprodukten mittels FTIR [171] und die Infrarot-Spektroskopie an Nanopartikeln [172] eingesetzt. Eine zweidimensionale Lavaldüse, die sich von den bisher vorgestellten achsensymmetrischen Typen unterscheidet, wurde von einer japanischen Gruppe zur Untersuchung von $\mathrm{UF}_{6}$ und dessen Clustern durch Massenspektrometrie und FTIR-Spektroskopie benutzt [173, 174,175, 176,177]. In einer weiteren Anwendung einer zweidimensionalen Lavaldüse wurde der Energietransfer zwischen einem Iodatom und molekularem Sauerstoff beobachtet [178,179]. Die Elektronenbeugung an Clustern in einer Lavaldüsenexpansion ist in Ref. [180,181] 
beschrieben. Der von einer Lavaldüse zur Verfügung gestellte uniforme Strahl ist also ein nicht nur für die Reaktionskinetik von Ionen und Radikalen exzellente Anwendungsmöglichkeit, sondern ist auch in anderen Gebieten vielseitig einsetzbar.

Eine für die Charakterisierung von Überschalldüsenexpansionen elementare Größe ist die Machzahl $M$ (Gl. 3.1). Sie ist eine Maß für die Umwandlung der Energie von zufälliger in gerichtete Bewegung während der Expansion.

$$
M=\frac{\text { hydrodynamische Flussgeschwindigkeit }}{\text { lokale Schallgeschwindigkeit }}
$$

Im Fall der freien Expansion nimmt die Machzahl mit dem Abstand von der Gasaustrittsöffnung zu, die Dichte wird geringer. Im Gegensatz dazu steht die Lavaldüsenexpansion, die einen in Machzahl, Dichte und Temperatur isentropen Gasfluss nach dem Düsenende liefert. Dieser Fluss befindet sich im thermischen, aber nicht unbedingt im chemischen Gleichgewicht, dadurch eröffnen sich diverse Möglichkeiten, z. B. die Bestimmung der Kinetik zahlreicher Reaktionen bei tiefen Temperaturen.

\subsection{Lavaldüse}

Um die benötigten Flusseigenschaften $(M, T, \rho)$ zu produzieren, muss eine spezielle Wandkontur für die Düsen nach den Gesetzen der Strömungsdynamik berechnet werden. Dabei ist zu beachten, dass für jede gewünschte Flusseigenschaft eine eigene Düse berechnet werden muss. Die grundlegenden Zusammenhänge zwischen Temperatur $T$, Dichte $\rho$, Druck $p$ und Machzahl $M$ werden durch die Gleichungen 3.2, 3.3 und 3.4 beschrieben, wenn die Moleküle auf eine konstante Machzahl beschleunigt werden. Dabei beziehen sich die mit 0 indizierten Größen auf die Reservoirbedingungen, die nicht-indizierten Größen auf Eigenschaften im Fluss.

$$
\begin{aligned}
\frac{T}{T_{0}} & =\left(1+\frac{\gamma-1}{2} M^{2}\right)^{-1} \\
\frac{\rho}{\rho_{0}} & =\left(1+\frac{\gamma-1}{2} M^{2}\right)^{\frac{1}{1-\gamma}} \\
\frac{p}{p_{0}} & =\left(1+\frac{\gamma-1}{2} M^{2}\right)^{\frac{\gamma}{1-\gamma}}
\end{aligned}
$$

Der Adiabatenkoeffizient $\gamma$ ist durch das Verhältnis der Wärmekapazitäten des Trägergases gegeben $\left(\gamma=\frac{C_{\mathrm{p}}}{C_{\mathrm{V}}}\right)$. Die in Gl. 3.2, 3.3 und 3.4 beschriebenen Größen finden 




Abbildung 3.3: Schema einer Lavaldüse

sich in Abb. 3.3 wieder. Die Lavaldüse besteht aus einem kurzen konvergenten Teil, in dem die Moleküle aus einem netto Stillstand - in der Stagnationsregion - auf Mach 1 beschleunigt werden. Die Form dieser konvergenten Region hat einen geringen Einfluss auf die Flusseigenschaften nach der Lavaldüse, einzig darf der Übergang zwischen konvergenter und divergenter Region keine Unstetigkeit aufweisen, weil sonst die Gefahr besteht, dass der Fluss der Wandkontur nicht mehr folgen kann und somit Verwirbelungen entstehen. Im engsten Teil der Düse, auch Düsenhals genannt, hat die Machzahl gerade den Wert 1 erreicht, die Geschwindigkeit des Gases entspricht der Schallgeschwindigkeit. Im divergenten Teil findet die Beschleunigung auf die endgültige Machzahl statt, sämtliche Expansionswellen quer zur Ausbreitungsrichtung werden entfernt. Dieser Sachverhalt ist auch in Abb. 3.2 verdeutlicht. In den folgenden Gleichungen ist der Zusammenhang zwischen dem Düsenquerschnitt $A$, dem statischen Druck $p$, der Flussdichte $\rho$, der hydrodynamischen Flussgeschwindigkeit $v$ und der Machzahl $M$ erläutert:

$$
\begin{aligned}
\frac{\mathrm{d} A}{A} & =\frac{\mathrm{d} p}{\rho v^{2}}\left(1-M^{2}\right) \\
\frac{\mathrm{d} v}{\mathrm{~d} p} & >0
\end{aligned}
$$

Aus obiger Gleichung ergibt sich, dass sich das Gas im konvergenten Teil der Lavaldüse mit Unterschall $(M<1)$, im divergenten Teil mit Überschall $(M<1)$ bewegt.

Der Fluss in einer Lavaldüse besteht aus einem isentropen Kern, an dessen Rand sich eine Grenzschicht befindet (vgl. Abb. 3.4), die erst später in die Berechnung der Wandkontur mit einbezogen wird. Zur Berechnung der Form des divergenten Lavaldüsenteils findet eine Aufteilung der Düse in verschiedene Regionen statt, wobei sich die Regionen in ihren Flusseigenschaften unterscheiden (vgl. Abb. (3.5)). 


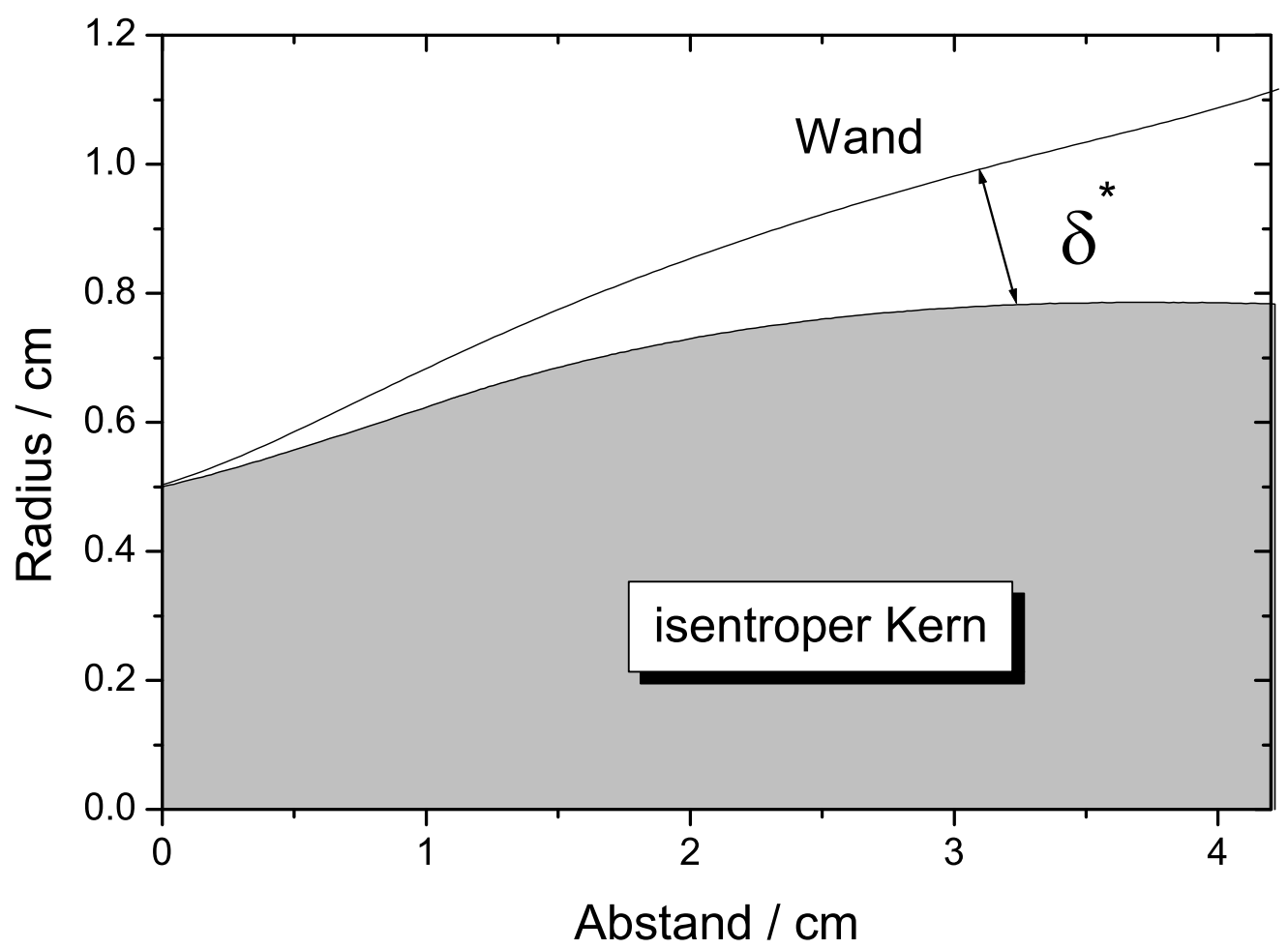

Abbildung 3.4: Isentroper Kern und Grenzschicht

In Punkt O befindet sich die Quelle des Flusses, von hier aus startet die Expansion. Innerhalb der Region I findet eine schnelle Beschleunigung auf ungefähr die Hälfte der endlichen Machzahl statt. Die Expansion in Region II lässt sich mit einer freien Expansion vergleichen, da hier alle Stromlinien radial von Punkt O ausgehen, die Strecke CD ist ein Kreisabschnitt mit dem Radius OD. Die Beschleunigung auf die endgültige Machzahl erreicht der Fluss dann in Region III, ab der Strecke EF verlaufen alle Stromlinien parallel zur Düsenachse (Abb. 3.5).

Die Berechnung des isentropen Kerns der Expansion erfolgt über die Methode der Charakteristiken, die für diese Arbeit verwendeten Düsen wurden nach der Methode von Moger und Ramsay berechnet. Die entsprechenden Gleichungen wurden von Atkinson $[158,159,160]$ in Microsoft QuickBasic ${ }^{\circledR}$ implementiert. Als Charakteristik wird eine Richtung einer konstanten Änderung beschrieben, sie beschreibt die Auswirkung einer Störung, z. B. wie sich die Änderung der Wandkrümmung im Fluss 


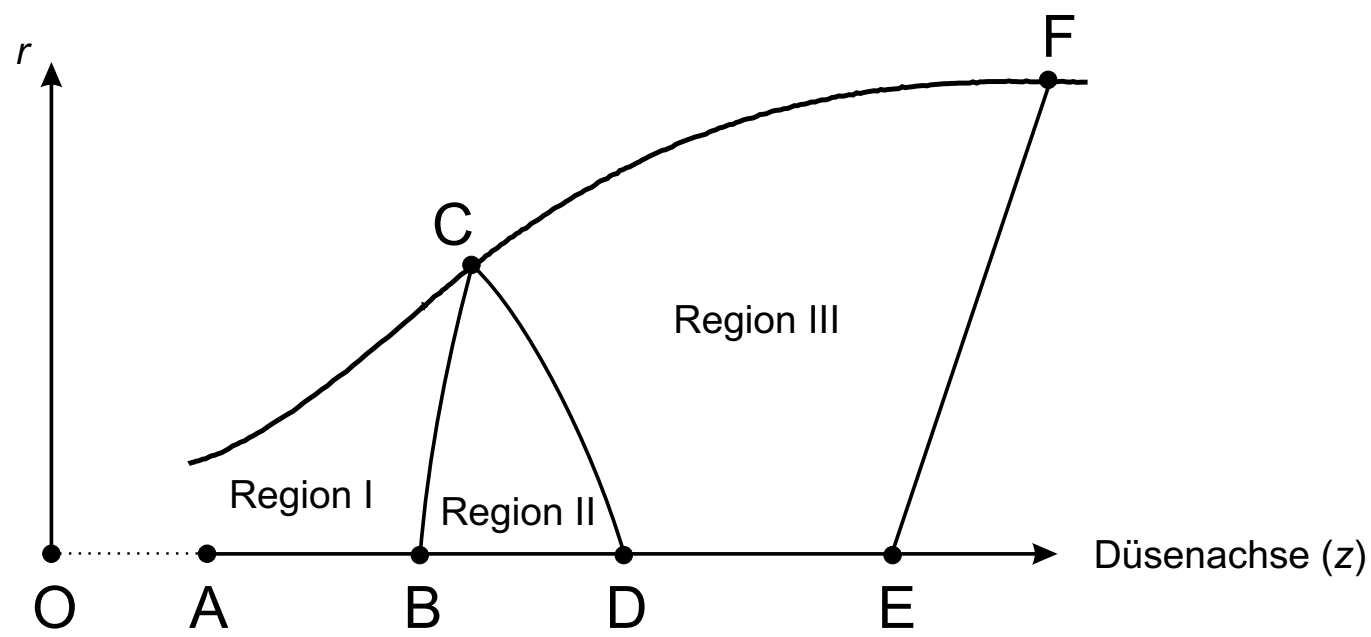

Abbildung 3.5: Regionen der Lavaldüse

fortpflanzt. Die Strecken BC, CD und EF in Abb. 3.5 sind Charakteristiken. Die Zusammenhänge zwischen den zylindrischen Koordinaten $z$ (Entfernung auf der Düsenachse), $r$ (radialer Abstand von der Düsenachse) und anderen Parametern kann durch zwei gewöhnliche Differentialgleichungen beschrieben werden:

$$
\begin{aligned}
\frac{\mathrm{d} r}{\mathrm{~d} z} & =\tan (\theta \mp a), \\
0 & =\mathrm{d} \theta \pm \frac{\cot (a)}{W} \mathrm{~d} W \mp \frac{\sin (\theta) \sin (a)}{\sin (\theta+a)} \frac{\mathrm{d} r}{r}
\end{aligned}
$$

mit $\theta$ als Flusswinkel (bezogen auf die Düsenachse, vgl. Abb. 3.6). Die oberen der 干bzw. \pm -Zeichen beziehen sich auf Charakteristiken mit negativer Steigung, die unteren entsprechend mit positiver Steigung. Der Machwinkel $a\left(a=\sin ^{-1}(1 / M)\right)$ beschreibt den Winkel zwischen Ausbreitungsrichtung und der Wand. $W$ ist das Verhältnis der hydrodynamischen Geschwindigkeit $V$ und thermodynamischer Grenzgeschwindigkeit $V_{\max }$ (die Geschwindigkeit, die der Fluss bei $M=\infty$ erreichen würde):

$$
\begin{aligned}
W^{2} & =\left(\frac{V}{V_{\max }}\right)^{2}=\frac{\gamma-1}{2} M^{2}\left(1+\frac{\gamma-1}{2} M^{2}\right)^{-1} \\
V_{\max } & =\left(\frac{2 \gamma}{\gamma-1} \frac{k_{\mathrm{B}} T_{0}}{m}\right)^{1 / 2}
\end{aligned}
$$

mit der Boltzmannkonstanten $k_{\mathrm{B}}$ und der Masse des Flussgases $m$. 


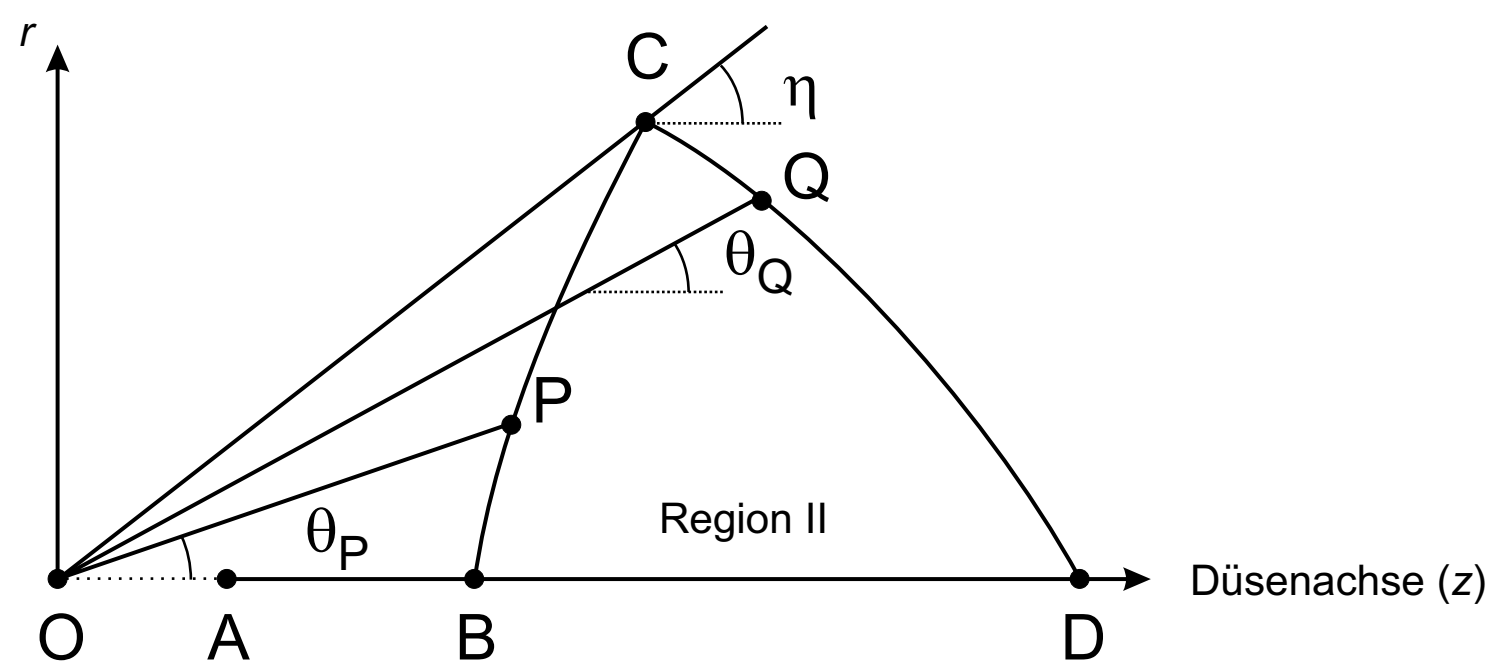

Abbildung 3.6: Region II der Lavaldüse

Als Startpunkt der Berechnungen dient die Region II, da hier alle Stromlinien vom Punkt $\mathrm{O}$ ausgehende gerade Linien sind. Ist nun die Machzahl am Ende der Region I gegeben ${ }^{1}$, so ist die Machzahl in jedem Punkt in Region II nur durch den Abstand vom Punkt O gegeben. Somit ergibt sich z. B. die Charakteristik EF aus der Tatsache, dass sowohl alle Stromlinien parallel zur Düsenachse sind, als auch die Machzahl der endgültigen Machzahl entspricht. Der integrierte Massenfluss bestimmt dann die Länge dieser Charakteristik. Der Massenfluss d $m$, der hier eine Erhaltungsgröße ist, wird durch Integration über die Charakteristik BC berechnet (Gl. 3.11).

$$
\mathrm{d} m=r W\left(1-W^{2}\right)^{1 /(\gamma-1)} \sin (a) \mathrm{d} l
$$

$l$ ist die Länge entlang eine Charakteristik. Die Grenzen von Region II werden durch die Prandtl-Meyer-Funktion $\psi$ bestimmt:

$$
\begin{aligned}
\psi & =\sqrt{\frac{\gamma+1}{\gamma-1}} \tan ^{-1}\left(\sqrt{\left(M^{2}-1\right) \frac{\gamma-1}{\gamma+1}}\right)-\tan ^{-1}\left(\sqrt{\left(M^{2}-1\right)}\right) \\
\psi_{\mathrm{P}} & =\psi_{\mathrm{B}}+2 \theta_{\mathrm{P}} \\
\psi_{\mathrm{Q}} & =\psi_{\mathrm{C}}+2\left(\eta-\theta_{\mathrm{Q}}\right) .
\end{aligned}
$$

$\psi_{\mathrm{P}}, \psi_{\mathrm{Q}}, \psi_{\mathrm{B}}$ und $\psi_{\mathrm{C}}$ sind die Werte der Prandtl-Meyer-Funktion an den Punkten $\mathrm{P}$, $\mathrm{Q}, \mathrm{B}$ und $\mathrm{C} ; \eta$ ist der Winkel am Punkt $\mathrm{C}$ (auf die Düsenachse bezogen), $\theta_{\mathrm{P}}$ und $\theta_{\mathrm{Q}}$

\footnotetext{
${ }^{1}$ für jede Berechnung zu wählen
} 
sind die Flusswinkel am Punkt C (vgl. Abb. 3.6). Der Wert der Prandtl-MeyerFunktion am Punkt B ist bekannt (nur durch $M$ bestimmt), die Flusswinkel ebenfalls (Stromlinien sind Geraden vom Punkt O ausgehend). Durch Unterteilung von $\eta$ in kleine Winkelelemente kann $\theta_{\mathrm{P}}$ berechnet werden, mit Hilfe dieser Werte wird die Machzahl entlang der Charakteristik BC gefunden. Eine analoge Prozedur ist für die Charakteristik CD durchzuführen. Nach der Bestimmung der Punkte auf den Charakteristiken BC, CD und EF und der Kenntnis der Flusseigenschaften auf der Düsenachse müssen nun die Koordinaten der begrenzenden Wand berechnet werden. Dazu werden ausgehend von vorhandenen Punkten im Fluss (z. B. auf CD oder der Düsenachse) neue Testpunkte errechnet, deren Flusseigenschaften mit denen der bekannten Punkte verglichen und optimiert werden. So werden die Punkte einer neuen Charakteristik von innen nach außen bestimmt, der tatsächliche Wandpunkt ist wiederum durch den integrierten Massenfluss gegeben, der mit dem totalen Massenfluss durch die Düse übereinstimmen muss. Der Durchmesser am Ende der Düse $A$ ist durch die Machzahl $M$ und den Durchmesser des Düsenhalses $A^{*}$ nach Gl. 3.15 gegeben.

$$
\frac{A}{A^{*}}=\frac{1}{M}\left[\left(\frac{2}{\gamma+1}\right)\left(1+\frac{\gamma-1}{2} M^{2}\right)\right]^{(\gamma+1) /[2(\gamma-1)]}
$$

Zur Vereinfachung dieser Berechnungen werden Wärmeübertragungs- und Viskositätseffekte zunächst außer Acht gelassen. Nachdem diese Prozedur für Region I und III durchgeführt wurde, ergibt sich ein Satz von relativen $(z, r)$-Koordinaten, die sich z. B. mit dem Durchmesser des Düsenhalses skalieren lassen, ohne dass sich dabei die Flusseigenschaften ändern. Das Bild des isentropen Flusses entspricht nur zum Teil der Realität. In der Praxis stellt sich der Fluss aus einer Lavaldüse folgendermaßen dar. Um einen isentropen Flusskern herum bildet sich durch Wärmeübertragungsund Viskositätseffekte eine Grenzschicht $\delta^{*}$, die berechnet werden muss, um die realen Wandkoordinaten zu erhalten. Dazu wird die Dicke der Grenzschicht bestimmt, in der die oben genannten Effekte auftreten. Dabei nimmt die Dicke der Grenzschicht mit zunehmender Dichte im Düsenstrahl ab. Dies ist auch der Grund, warum die Dichten in Lavaldüsenexpansionen auf einen Bereich über $10^{16} \mathrm{~cm}^{-3}$ limitiert sind, unterhalb wird die Grenzschicht so dick gegenüber dem isentropen Kern, dass keine kinetischen Experimente in einem thermisch equilibrierten kalten Ensemble mehr möglich sind. Bei hohen Flussdichten $\left(\rho>10^{18} \mathrm{~cm}^{-3}\right)$ tritt eher das Problem auf, den Düsenstrahl mit dem Hintergrunddruck „einzufassen“, es gelingt immer schlechter, alle Expansionswellen zu entfernen. Als Konsequenz einer zu hohen Strahldichte treten vermehrt Oszillationen in der Ausbreitungsrichtung auf. 


\subsection{Aufbau}

\subsubsection{Vakuumkammer}

Die Messungen wurden in einem Edelstahl-Vakuumkammer (CF-250-Kreuz) durchgeführt. Das Vakuum wurde durch eine Pumpenkombination aus einer Wälzkolbenpumpe (BOC Edwards EH500) und einer Drehschieberpumpe (BOC Edwards E2M80) mit einem Gesamtdurchsatz von $400 \mathrm{~m}^{3} / \mathrm{h}$ aufrecht erhalten und erreichte eine Wert von ca. $10^{-5}$ mbar. Der Kern der Vakuumapparatur besteht aus einem auf ein Metallrohr aufgeschraubten Aluminiumblock, in dessen Mitte sich das Gasreservoir für die Expansion befindet. Das Gasreservoir hat ein Volumen von $0.5 \mathrm{~cm}^{-3}$ und wird von zwei gegenüberliegenden Gaseinlässen gefüllt.

Die Zuführung des Precursors für die Hydroxylradikale, Wasserstoffperoxid $\left(\mathrm{H}_{2} \mathrm{O}_{2}\right)$, erfolgt, indem das Trägergas (Stickstoff) durch eine Waschflasche mit $\mathrm{H}_{2} \mathrm{O}_{2}$ und anschließend durch einen kalibrierten Durchflussmesser (Tylan FC 260, $1 \mathrm{slm}$ ) geleitet wurde. Die Erzeugung des Gaspulses erfolgte im Fall des mit $\mathrm{H}_{2} \mathrm{O}_{2}$ angereicherten Badgases mit Hilfe einer umgebauten PKW-Einspritzdüse (Bosch). Die Zufuhr des mit Stickstoff in einer Edelstahlflasche (Messer-Griesheim) vorgemischten Reaktanden erfolte über eine Magnetventil (General Valve Series 9, Öffnung: $150 \mu \mathrm{m}$ ) und einen vorgeschalteten ebenfalls kalibrierten Durchflussmesser (Tylan FC 260, $0.1 \mathrm{slm}$ ). Die Dauer der beiden Gaspulse betrug ca. $7 \mathrm{~ms}$ (vgl. Abb. 3.7). Die Kalibration der Durchflussmesser erfolgte über die Beobachtung des zeitabhängigen Druckanstiegs während der Expansion des zu kalibrierenden Gases in ein bekanntes Volumen.

Die Lavaldüsenform wurde mit Hilfe eines Programmes von Prof. M. Smith [160] berechnet und in der Feinmechanikwerkstatt des Institutes für Physikalische Chemie in Göttingen auf einer CNC-Drehbank anhand eines Satzes aus Abstand-DurchmesserKoordinaten (ca. 300 Punkte) aus Aluminium gefertigt.

\subsubsection{Laser und optischer Aufbau}

Zur Dissoziation des Precursors $\left(\mathrm{H}_{2} \mathrm{O}_{2}\right)$ wurde ein Excimer-Laser (Lambda-Physik EMG-101) bei zwei verschiedenen Wellenlängen benutzt. Zu Beginn der Experimente wurde der Excimer-Laser bei $193 \mathrm{~nm}$ (ArF), später bei $248 \mathrm{~nm}$ (KrF) betrieben. Für den Betrieb bei 193 nm wurde der Excimer-Laser mit 160 mbar einer Fluor/Helium-Mischung (5\%), 300 mbar Argon und Helium auf insgesamt 2200 mbar gefüllt. Sollte der Laser bei $248 \mathrm{~nm}$ betrieben werden, so wurde folgende Mischung 


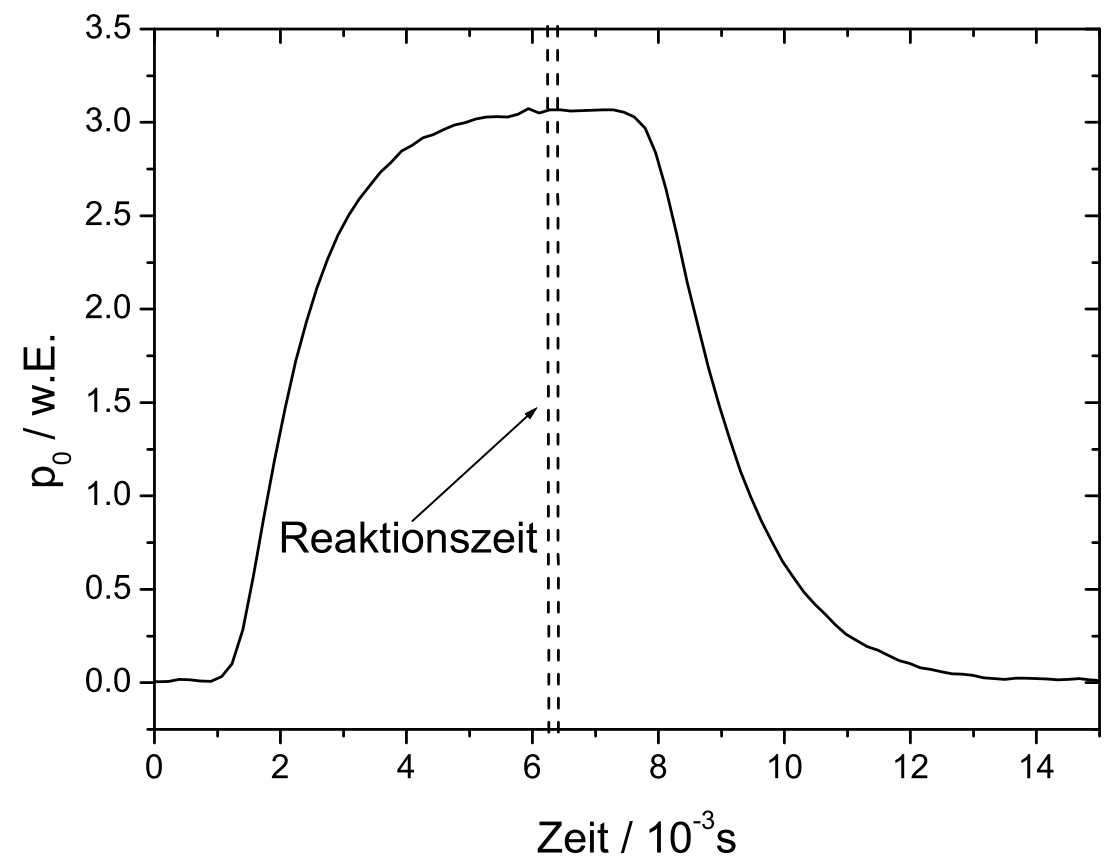

Abbildung 3.7: Druckpuls: Öffnungszeit ca. $7 \mathrm{~ms}$, Reaktionszeit max. $200 \mu \mathrm{s}$

eingefüllt: 120 mbar der Fluor/Helium-Mischung (5\%), 150 mbar Argon und Helium (insgesamt $2500 \mathrm{mbar}$ ). In beiden Fällen betrug die Laserpulsdauer ca. $10 \mathrm{~ns}$, die maximalen Laserpulsenergien betrugen $150 \mathrm{~mJ}$ (193 nm) bzw. $190 \mathrm{~mJ}(248 \mathrm{~nm})$. Zum Nachweis der erzeugten Hydroxylradikale mittels laserinduzierter Fluoreszenz (LIF) wurde die zweite Harmonische eines Farbstofflasers (Lambda-Physik Scanmate 2E), der durch die zweite Harmonische eines Nd:YAG-Lasers (Spectra-Physics GCR-150) optisch gepumpt wurde, genutzt. Die Fundamentalwellenlänge wurde über ein Pellin-Broca-Prisma abgetrennt. Bei $532 \mathrm{~nm}$ betrug die verwendete Pulsenergie des Nd:YAG-Lasers ca. 40 mJ. Für den benötigten Spektralbereich wurde der Oszillator des Farbstofflasers mit einer Lösung von Rhodamin 6G in Ethanol befüllt, die Lösung wurde für den Verstärker auf $1 / 3$ vedünnt. Die Pulsenergie bei $563.8 \mathrm{~nm}$ betrug ca. $10 \mathrm{~mJ}$, die Effizienz des Farbstoffes ergab also 25\%.

Zur Anregung der Fluoreszenz wurde die verdoppelte Wellenlänge des Farbstofflasers auf $281.915 \mathrm{~nm}\left(\mathrm{Q}_{1}(1)\right.$ - Linie) eingestellt, welche einer Anregung aus dem Schwingungsgrundzustand des elektronischen Grundzustandes in den ersten angeregten Schwingungszustand des elektronisch angeregten Zustandes $(1 \leftarrow 0)$ ent- 


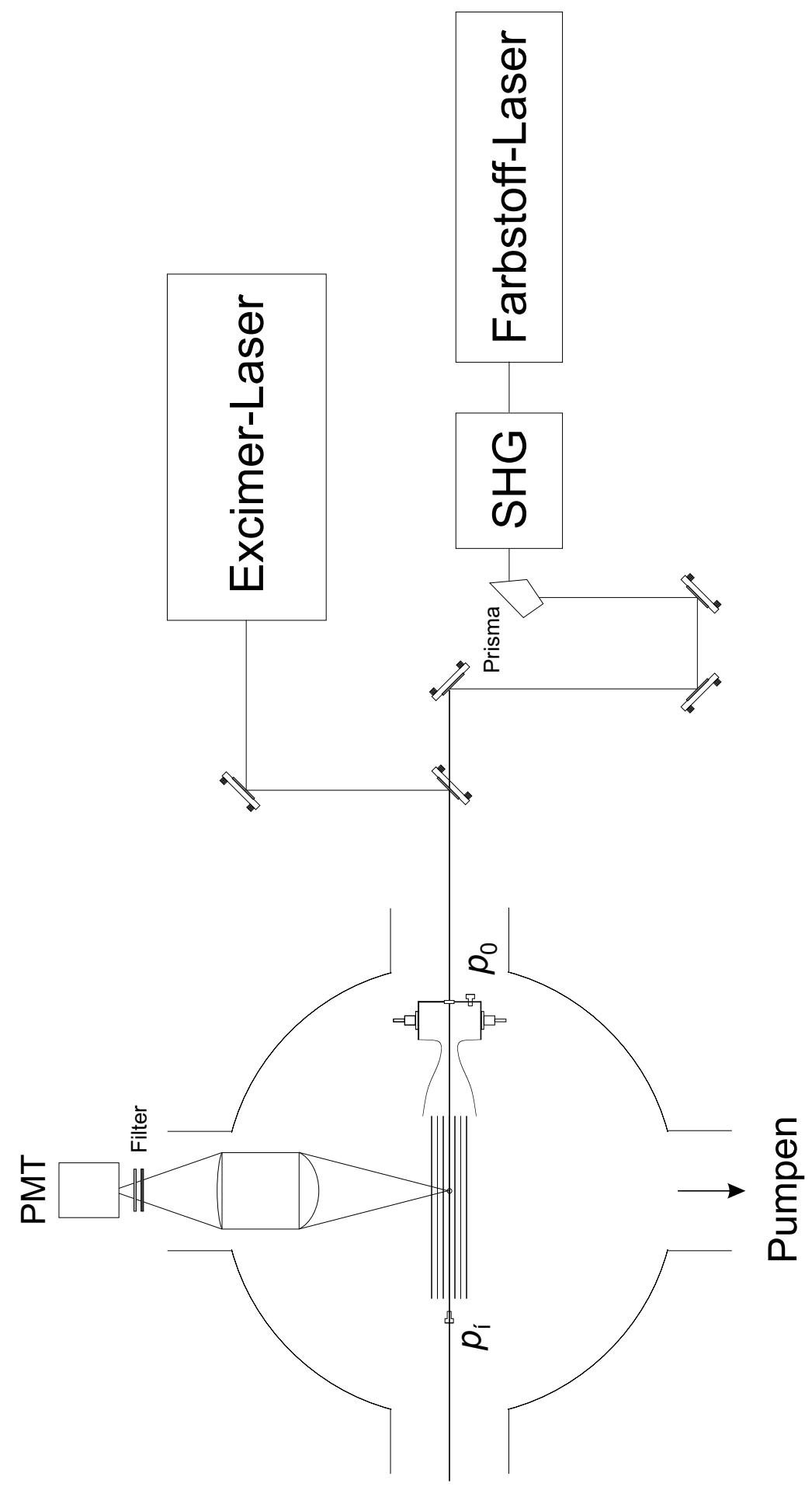

Abbildung 3.8: Aufbau der gepulsten CRESU-Apparatur 
spricht. Weiterhin konnten die Hydroxylradikale im ersten angeregten Schwingungszustand des elektronischen Grundzustandes beobachtet werden, dazu wurde die Anregung des $(2 \leftarrow 1)$-Übergangs bei einer Wellenlänge von $288.233 \mathrm{~nm}\left(\mathrm{Q}_{1}(1)-\right.$ Linie $)$ benutzt. Beide Laserstrahlen wurden über hochreflektierende dielektrische Spiegel kollinear in die Vakuumkammer eingekoppelt. Als Fenster für die Vakuumkammer wurden zwei $2 \mathrm{~mm} \times 50 \mathrm{~mm}$ Quarzglasplatten, für das Abdichten des Gasreservoirs innerhalb der Kammer eine $2 \mathrm{~mm} \times 14 \mathrm{~mm}$ Quarzglasplatten verwendet.

\subsection{Chemische Substanzen}

Das als Precursor für die Hydroxylradikale verwendete Wasserstoffperoxid $\left(\mathrm{H}_{2} \mathrm{O}_{2}\right)$ wurde von der Firma Solvay Interox in einer Reinheit von $70 \%$ bezogen und über Nacht mit Stickstoff durch eine Waschflasche geleitet und dadurch auf ca. $90 \%$ aufkonzentriert. Stickstoff (Messer Griesheim, Reinheit 99.996\%) und die Reaktanden Ethin (99.6\%), Ethen (99.5\%), Propen (99.5\%) (alle Messer Griesheim) sowie 2-Methyl-1,3-Butadien (Isopren, Fluka, $\geqslant 98 \%$ ) wurden ohne weitere Reinigung verwendet.

\subsection{Signalaufnahme und Datenverarbeitung}

Die von der zweiten Harmonischen eines Farbstofflasers angeregte Fluoreszenz der Hydroxylradikale wurde durch ein Linsensystem und eine Filterkombination mit einem Photomultiplier (Hochspannung: $U=1.25 \mathrm{kV}$ ) gesammelt. Das Linsensystem bestand zum einen aus einer Sammellinse (plankonvex, $f=50 \mathrm{~mm}$ ), die sich in einem Abstand von $50 \mathrm{~mm}$ zur Mitte des isentropen Gasstrahls befand, um die isotrop emittierte Fluoreszenz zu sammeln. Zum anderen wurde die so parallelisierte Strahlung mit Hilfe einer Sammellinse (plankonvex, $f=200 \mathrm{~mm}$ ) auf die photoempfindliche Schicht des Photomultipliers fokussiert. Direkt vor dem Photomultiplier befanden sich sowohl ein Ultraviolettfilter (UG11, Schott) als auch ein Interferenzfilter $\left(\lambda=306 \mathrm{~nm}, \mathrm{FWHM}=8.5 \mathrm{~nm}, T_{\max }=37 \%\right.$, Dr. Hugo Anders $)$, sodass die Fluoreszenz nur in einem spektral schmalen Fenster detektiert wurde, um sowohl direkte Strahlung des Photolyse(Excimer)- und Anregungslasers(Farbstofflaser) sowie deren Reflektionen an Metalloberflächen als auch Tageslicht auszublenden.

Das vom Photomultiplier ausgegebene Signal wurde von einem Boxcar-Integrator (EG\&G Parc 4141B) über einen Zeitraum von $150 \mu$ s mit einer Verzögerung von 
wenigen 100 ns nach der Fluoreszenzanregung integriert und über eine A/D-Wandlerkarte in einen PC übermittelt und dort mit einem Messprogramm verarbeitet. Die zeitliche Entwicklung der Hydroxylradikalkonzentration wurde jeweils mit einer negativen Verzögerungszeit von $10 \mu$ s und einer Zeitauflösung von $1 \mu$ s über eine Zeit von maximal $200 \mu$ s aufgenommen, jeder Messpunkt wurde zur Verbesserung des Signal/Rausch-Verhältnisses zwischen 30 und 100 mal gemittelt. Die so erhaltenen Kinetiken wurden mit dem folgenden Modell angepasst. Dazu werden zunächst alle Prozesse betrachtet, die während des Experimentes ablaufen:

$$
\begin{array}{lc}
\text { Photolyse: } & \mathrm{H}_{2} \mathrm{O}_{2}+h \nu \rightarrow 2 \mathrm{OH}^{*} \\
\text { Relaxation: } & \mathrm{OH}^{*}+\mathrm{M} \stackrel{k_{1}^{\prime}}{\longrightarrow} \mathrm{OH}+\mathrm{M} \\
\text { Reaktion: } & \mathrm{OH}+\mathrm{X} \stackrel{k_{2}^{\prime}}{\longrightarrow} \mathrm{OHX}
\end{array}
$$

Dabei ist die Relaxation der Prozess, bei dem die nach der Photolyse „heißen“ Hydroxylradikale $\left(\mathrm{OH}^{*}\right)$ der thermodynamischen Gleichgewichtsbesetzung zugeführt werden, X steht für die Reaktanden (Ethin, Ethen, Propen und Isopren). Aus den Gleichungen 3.16, 3.17 und 3.18 ergibt sich folgendes Geschwindigkeits-Zeit-Gesetz für die Hydroxylradikalkonzentration:

$$
\frac{\mathrm{d}[\mathrm{OH}]}{\mathrm{d} t}=k_{1}^{\prime}[\mathrm{OH}]^{*}[\mathrm{M}]-k_{2}^{\prime}[\mathrm{OH}][\mathrm{X}] .
$$

Unter den gegebenen experimentellen Bedingungen sind sowohl die Konzentration des Badgases [M] als auch die Konzentration der Reaktanden [X] wesentlich größer als die der Hydroxylradikale (siehe unten), so dass sie in die Geschwindigkeitskonstanten $k_{1}^{\prime}$ bzw. $k_{2}^{\prime}$ einbezogen werden können, es handelt sich um eine Kinetik pseudo-erster Ordnung:

$$
\frac{\mathrm{d}[\mathrm{OH}]}{\mathrm{d} t}=k_{1}[\mathrm{OH}]^{*}-k_{2}[\mathrm{OH}] .
$$

Die Integration von Gleichung 3.20 ergibt

$$
[\mathrm{OH}](t)=\frac{[\mathrm{OH}]_{0} k_{2}}{k_{1}-k_{2}}\left[\exp \left(-k_{1} t\right)-\exp \left(-k_{2} t\right)\right]
$$

dabei ist $[\mathrm{OH}]_{0}$ die Anfangskonzentration der Hydroxylradikale $(t=0)$. Die Anpassung der experimentellen zeitabhängigen Hydroxylradikalkonzentration an Gleichung 3.21 erfolgte über einen Levenberg-Marquardt-Algorithmus mit dem Programm Origin ${ }^{\circledR}$ (Microcal).

Zur Verifizierung der Bedingungen pseudo-erster Ordnung wurde die mittlere Hydroxylradikalkonzentration bestimmt und mit den verwendeten Konzentrationen 
der Reaktionspartner verglichen. Die Konzentration der Hydroxylradikale wurde über eine relative Methode bestimmt, da die Konzentration des Wasserstoffperoxid in der Waschflasche, durch die das Trägergas geleitet wurde, nicht bekannt ist. Dazu wurde eine Mischung bekannter Konzentration aus Wasserdampf und Trägergas hergestellt und die Intensität des Fluoreszenzsignals gemessen. Daraus kann die Konzentration der aus dem Wasserdampf erzeugten Hydroxylradikale bestimmt werden. Zunächst wird die Fluenz (Energie/Fläche) des Excimer-Lasers berechnet, die theoretisch benötigt wird, um alle vorliegenden Wassermoleküle zu dissoziieren. Diese Fluenz $\Psi_{100 \%}$ ergibt sich aus dem Quotienten der Energie eines Photons ch/ $\lambda$, der Quantenausbeute $\Phi$ und dem Absorptionsquerschnitt $\sigma$ des Precursors bei der benutzten Wellenlänge $\lambda$, die entsprechenden Daten sind in Ref. [182, 183, 184, 185] aufgeführt.

$$
\Psi_{100 \%}=\frac{c h}{\lambda} \frac{1}{\sigma} \cdot \Phi
$$

Dabei ist $c$ die Lichtgeschwindigkeit und $h$ die Planck'sche Konstante. Über die tatsächlich in den Experimenten verwendete Fluenz $\Psi_{\text {exp }}$ wird die OH-Konzentration bestimmt.

$$
N_{\mathrm{OH}}=\frac{\Psi_{\exp }}{\Psi_{100 \%}} \cdot N_{\mathrm{H}_{2} \mathrm{O}}
$$

Das Verhältnis der Fluoreszenzintensitäten der aus unterschiedlichen Vorläufern $\left(\mathrm{H}_{2} \mathrm{O}_{2}\right.$ bzw. $\left.\mathrm{H}_{2} \mathrm{O}\right)$ erzeugten OH-Radikale gibt unter gleichen Bedingungen im Düsenstrahl das Verhältnis der Konzentrationen wieder, allerdings unter der Beachtung der verschiedenen Absorptionsquerschnitte und Quantenausbeuten. Unter gewöhnlichen experimentellen Bedingungen wurde eine $\mathrm{OH}$-Radikalkonzentration von $9 \cdot 10^{11} \mathrm{~cm}^{-3}$ abgeschätzt. Typische Reaktandenkonzentrationen betrugen ca. $1 \cdot 10^{15} \mathrm{~cm}^{-3}$, so dass ein genügend großer Überschuss gegenüber den Radikalen gegeben war, die Bedingungen pseudo-erster Ordnung war also garantiert.

Abschließend soll noch der Bildungsgrad von Photoprodukten der Reaktanden überprüft werden, damit ausgeschlossen werden kann, dass ein Teil der Reaktanden nicht schon zu Beginn der Reaktion nicht mehr vorhanden ist oder die OH-Radikale mit den Photoprodukten reagieren. Der Absorptionsquerschnitt von Ethin bei der verwendeten Excimer-Wellenlänge von $193 \mathrm{~nm}$ befindet sich in einem Bereich von (716) $\cdot 10^{-20} \mathrm{~cm}^{2}[186,187]$. Bei einer mittleren Teilchenkonzentration des Ethins von $1 \cdot 10^{15} \mathrm{~cm}^{-3}$ und einer Absorptionsweglänge von ca. $10 \mathrm{~cm}$ (Länge des uniformen Düsenstrahls) ergibt sich eine Absorption $<0.1 \%$, die Reaktion wird also nicht durch die Absorption des Ethins beeinflusst. Analoge Berechnungen lassen sich für Ethen, Propen und Isopren durchführen und liefern ähnliche, eher noch geringere Absorptionen. 


\subsection{Zeitliche Steuerung}

Die zeitliche Steuerung des Experimentes wurde durch zwei Delay-Generatoren (Stanford Systems DG535) realisiert. Die verschiedenen Impulse und ihre zeitliche Verzögerung sind in Abb. 3.9 aufgeführt. Zum Zeitnullpunkt eines jeden Experimentes, das mit einer Reptitionsrate von $10 \mathrm{~Hz}$ ausgeführt wurde, werden die beiden gepulsten Düsen angesteuert und geöffnet (Düsenöffnung). Nach ca. 6 ms wird dann mit variabler Verzögerungszeit der Excimerlaser gefeuert (Excimer Puls), zu einem festen Zeitpunkt ca. $200 \mu$ s danach der Nd:YAG- und damit auch der Farbstofflaser (Q-Switch des Nd:YAG). Ungefähr $200 \mathrm{~ns}$ später wird der Boxcar-Integrator aufgeladen (Boxcar-Fenster), nach insgesamt $6.7 \mathrm{~ms}$ liest der Analog/Digital-Wandler das Boxcar-Signal aus (Auslese-Puls A/D-Wandler), der Messzyklus schliefst mit der Entladung des Boxcar-Integrators (Löschpuls für Boxcar) und damit der Löschung der Information. Es bleiben also ca. $90 \mathrm{~ms}$, um die Messzelle zwischen zwei Zyklen zu evakuieren.

\subsection{Charakterisierung der Expansion}

Um zu garantieren, dass die kinetischen Experimente unter konstanten, thermisch equilibrierten Bedingungen $(T, \rho, M)$ stattfinden, wurden diese getestet und mit den durch die Berechnung der Lavaldüsenform vorgegebenen Parametern verglichen. Sehr wichtig für die Ausbildung eine uniformen Flusses nach dem Düsenende ist die Einstellung eines konstanten Hintergrunddrucks in der Vakuumkammer. Diese Tatsache steht im deutlichen Gegensatz zu einer freien Expansion, in der der Hintergrunddruck in der Reaktionskammer so niedrig wie möglich sein sollte, da sich sonst durch den Aufprall der Expansion auf den Hintergrunddruck eine Stoßfront ausbilden kann. Diese so genannte Mach'sche Scheibe begrenzt die Länge der nutzbaren freien Expansion, die Länge des nutzbaren Lavaldüsenstrahls wird eher durch eine zu größeren Abständen von der Öffnung dicker werdende Grenzschicht bestimmt. Bei Lavaldüsen dient der Hintergrunddruck als Einfassung des uniformen Strahls; Stromlinien, die eine gewisse Komponente quer zur Ausbreitungsrichtung besitzen, werden so eliminiert. Der optimale Hintergrunddruck entspricht dem statischen Druck im Fluss und wird zur Optimierung des uniformen Lavaldüsenstrahls verwendet. Experimentell wird die Einstellung des Hintergrunddruckes über ein Feindosierventil realisiert, das kontinuierlich eine bestimmte Menge Gas in die Vakuumkammer einlässt. 
Excimer Puls

Löschpuls für Boxcar

Q-Switch des Nd:YAG

Auslese-Puls A/D-Wandler

Boxcar-Fenster

Stagnationsdruck

Düsenöffnung

\begin{tabular}{cccccccc}
\hline 1 & 1 & 1 & 1 & 1 & 12 \\
& 2 & 4 & 6 & 8 & 10 & 12 \\
& $\mathrm{t} / 10^{-3} \mathrm{~s}$ & & &
\end{tabular}

Abbildung 3.9: Zeitliche Steuerung des Experimentes 
Zur weiteren Erklärung der Charakterisierung der Düsenbedingungen werden noch einmal die Gleichungen 3.2, 3.3 und 3.4 aufgeführt:

$$
\begin{aligned}
& \frac{T}{T_{0}}=\left(1+\frac{\gamma-1}{2} M^{2}\right)^{-1} \\
& \frac{\rho}{\rho_{0}}=\left(1+\frac{\gamma-1}{2} M^{2}\right)^{\frac{1}{1-\gamma}} \\
& \frac{p}{p_{0}}=\left(1+\frac{\gamma-1}{2} M^{2}\right)^{\frac{\gamma}{1-\gamma}} .
\end{aligned}
$$

Zur Bestimmung der Machzahl kann z. B. Gl. 3.2 herangezogen werden, d.h. die Temperatur im isentropen Fluss $(T)$ muss bestimmt werden, die Temperatur in der Stagnationsregion $\left(T_{0}\right)$ entspricht im allgemeinen der Raumtemperatur, wenn das Reservoir und die Lavaldüse nicht gekühlt werden. Die Bestimmung von $T$ durch konventionelle Thermometer gestaltet sich sehr schwierig, da das Gas in Bewegung ist und so die Kontaktzeit zwischen Gas und Thermometer sehr kurz ist. Eine wesentlich elegantere Methode ist die Bestimmung der Temperatur im Fluss durch die Ermittlung der temperaturabhängigen Besetzung von Rotationsniveaus. Experimentell wird ein rotationsaufgelöstes LIF-Spektrum bei einer festen Verzögerungszeit zwischen Photolyse und Fluoreszenzanregung aufgenommen. Dazu bietet sich im Fall der vorliegenden Arbeit das Rotationspektrum der Hydroxylradikale an, welches wohl bekannt und ausreichend charakterisiert ist. Um nun die Temperatur zu ermitteln, muss ein Zweig (Serie von optischen Übergängen zwischen zwei Rotationsniveaus mit gleicher Änderung der Quantenzahl) des Spektrums möglichst vollständig detektiert werden. Aus dem Verhältnis der Linienintensitäten, die mit der Besetzung der Ausgangsniveaus korrelieren, kann nach folgender Methode eine Temperatur abgeleitet werden. Die Intensität einer Fluoreszenzemission $I_{n m}$ eines Übergangs $n \rightarrow m$ hängt in folgender Weise von verschiedenen Größen ab:

$$
I_{n m}=N_{n} A_{n m} \cdot h \nu_{n m}
$$

In Gl. 3.24 indiziert $n$ das Ausgangs-, $m$ das Endniveau, $A_{n m}$ ist die Übergangswahrscheinlichkeit für Übergang zwischen $n$ und $m, N_{n}$ ist die Anzahl der Moleküle im Anfangszustand. Die Besetzung des Ausgangsniveaus $N_{n}$ ist im Fall einer isentropen Lavaldüsenexpansion durch eine Boltzmann-Verteilung gegeben (Gl. 3.25).

$$
\frac{N_{n}}{N_{0}}=\exp \frac{-E_{n}}{k_{\mathrm{B}} T}
$$

mit der Boltzmannkonstanten $k_{\mathrm{B}}$ und der Energie des Anfangszustandes $E_{n}$. 
Bei einer geeigneten Auftragung der gemessenen Linienintensitäten gegen die Energie des unteren Niveaus (Gl. 3.26) ergibt sich die Steigung der Geraden als $\left(-\frac{1}{k_{\mathrm{B}} T}\right)$.

$$
\ln \frac{I_{m n}}{A_{m n}}=\text { const. }-\frac{E_{n}}{k_{\mathrm{B}} T}
$$

In Abb. 3.10 ist das Rotationsspektrum des $\mathrm{OH}$-Übergangs von $X^{2}\left(v^{\prime \prime}=0\right)$ nach $A^{2}\left(v^{\prime}=1\right)$ gezeigt, in Abb. 3.11 der Ausschnitt $\left(S_{21}(K)\right.$-Zweig), der zur Temperaturbestimmung herangezogen wurde.

Abb. 3.12 zeigt die entsprechende Auftragung und Abb. 3.13 schließlich die nach dieser Methode bestimmte Temperatur in Abhängigkeit des Abstandes des Lavaldüsenendes vom LIF-Beobachtungspunkt direkt unter der Sammellinse des Photomultipliers. Die Übergangswahrscheinlichkeiten wurden aus Ref. [188] entnommen.

Ein andere, komplementäre Bestimmung der Flusstemperatur ist durch die experimentelle Bestimmung der Größen in Gl. 3.4 bzw. Gl. 3.3 gegeben. Wird also der Druck bzw. die Teilchenzahldichte sowohl im Reservoir als auch im Fluss bestimmt, so kann mit Hilfe der bestimmten Machzahl die Temperatur errechnet werden. Die Messung des Druckes in der Stagnationsregion $p_{0}$ ist in Abb. 3.8 gezeigt; in den das Reservoir beherbergenden Block ist ein Druckaufnehmer (Omega PX140-14VDV bzw. PX140-28VDV und PX005-GV für die verschiedenen Druckbereiche) eingelassen. Die Messung des Druckes im Fluss gestaltet sich etwas schwieriger, da es sich bei $p$ in Gl. 3.4 um einen statischen Druck handelt, das Gas sich aber mit einer bestimmten Geschwindigkeit fortbewegt. Bei Bestimmung des Druckes entgegen der Flussrichtung (wie in Abb. 3.8 angedeutet) handelt es sich also um eine dynamische Messung, es wird ein Aufpralldruck $p_{\mathrm{i}}$ (i für impact=Aufprall) gemessen. Mit Hilfe der Rayleigh-Pitot-Formel (3.27) kann $p_{\mathrm{i}}$ in den statischen Druck $p$ umgerechnet werden.

$$
\frac{p_{\mathrm{i}}}{p}=\left[\frac{(\gamma+1) M^{2}}{(\gamma-1) M^{2}+2}\right]^{\frac{\gamma}{\gamma-1}}\left[\frac{\gamma+1}{2 \gamma M^{2}-\gamma+1}\right]^{\frac{1}{\gamma-1}}
$$

Um nun optimale Bedingungen für jede gefertigte Lavaldüse zu finden, werden alle Bedingungen $\left(T_{0}, p_{0}\right)$ so eingestellt, dass sie den berechneten Werten entsprechen, der Hintergrundruck dient als Optimierungshilfe, um z. B. Oszillationen in der Expansion zu vermeiden. Mit Hilfe von Gl. 3.27, die $p$ liefert, und der Messung von $p_{0}$ kann die Machzahl $M$ berechnet werden. Aus der Machzahl, der Kenntnis von $\rho_{0}$ (aus $p_{0}$ ) und $T_{0}$ können dann die Flusstemperatur $T$ und die Dichte im Fluss $\rho$ bestimmt werden. Um die Uniformität des Düsenstrahls zu testen, wurden Messungen 


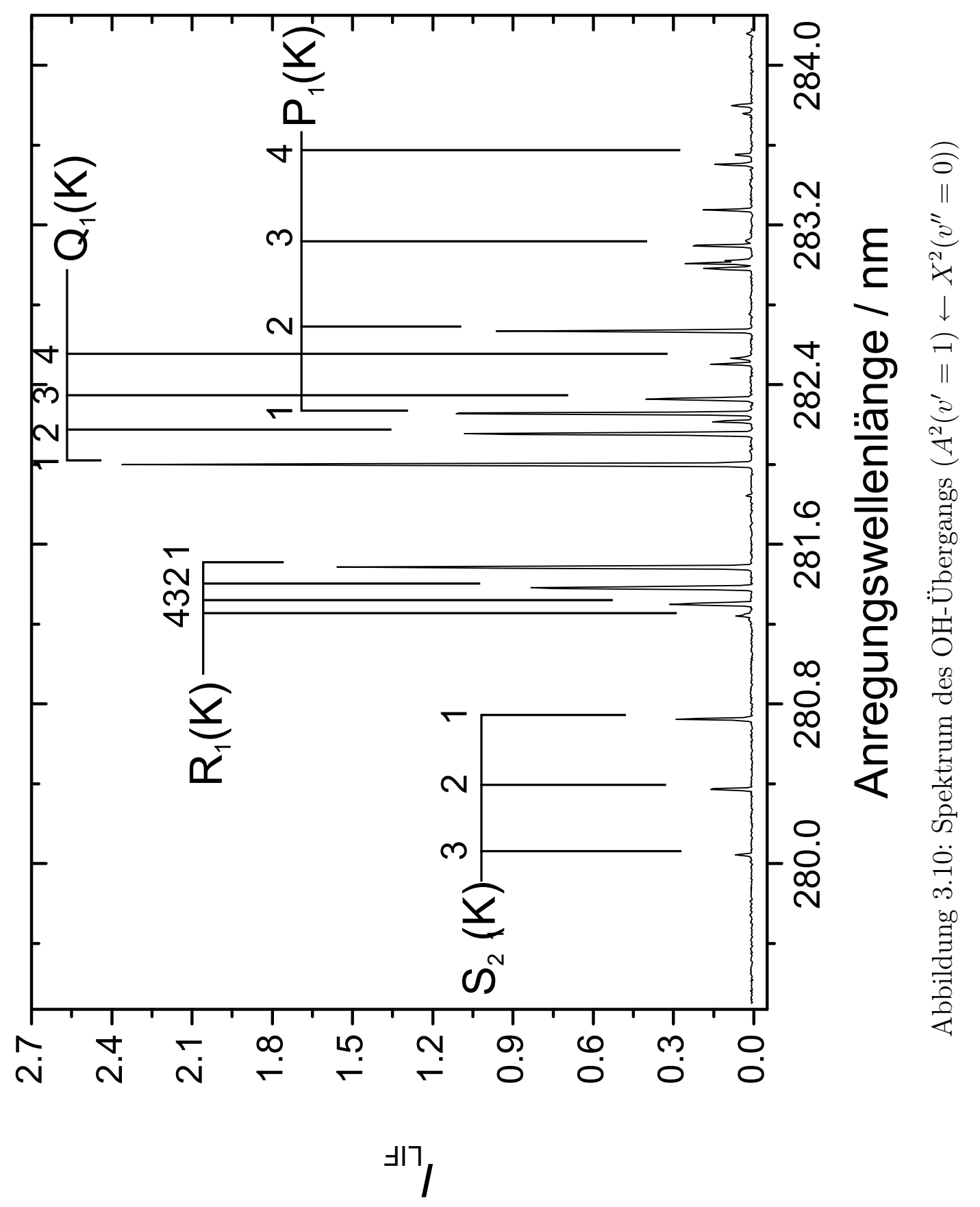




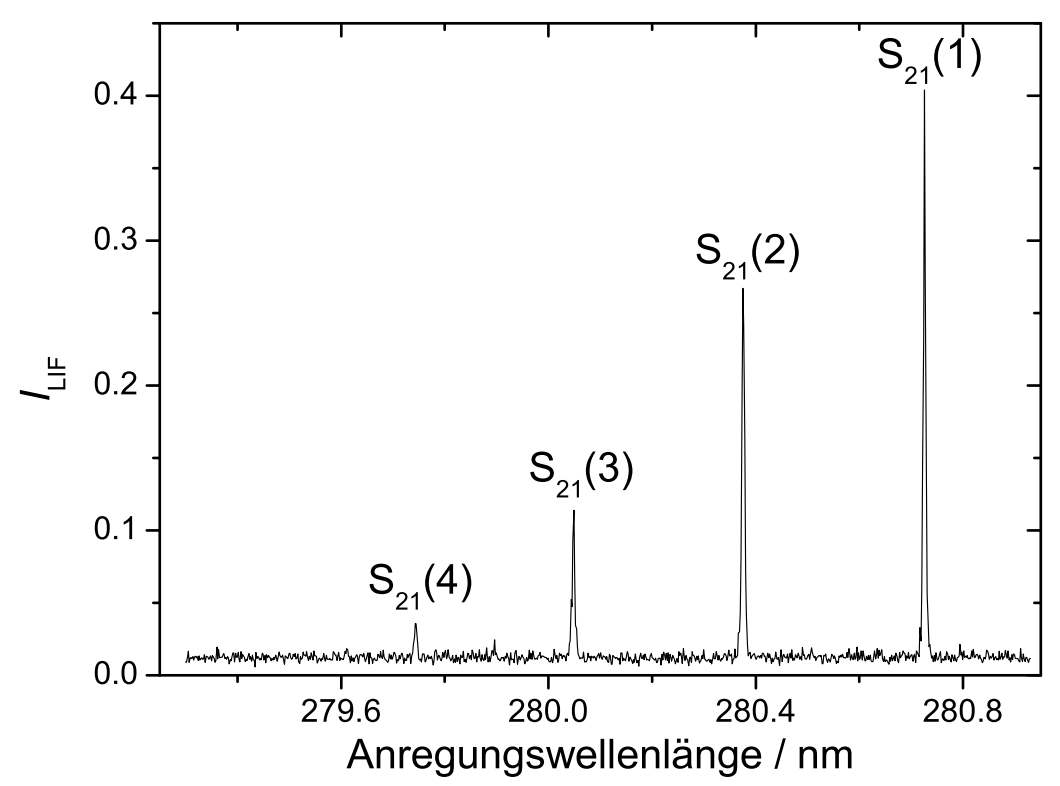

Abbildung 3.11: $S_{21}(K)$-Zweig

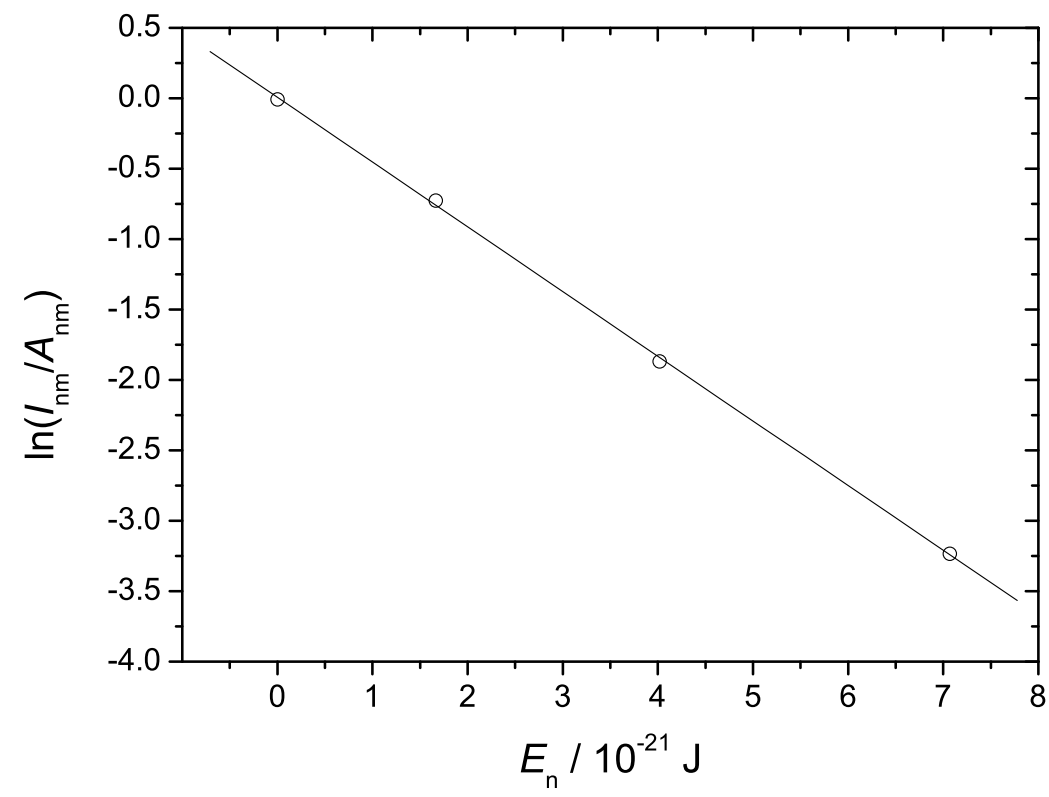

Abbildung 3.12: Auftragung zur Ermittlung der Flusstemperatur nach Gl. 3.26 


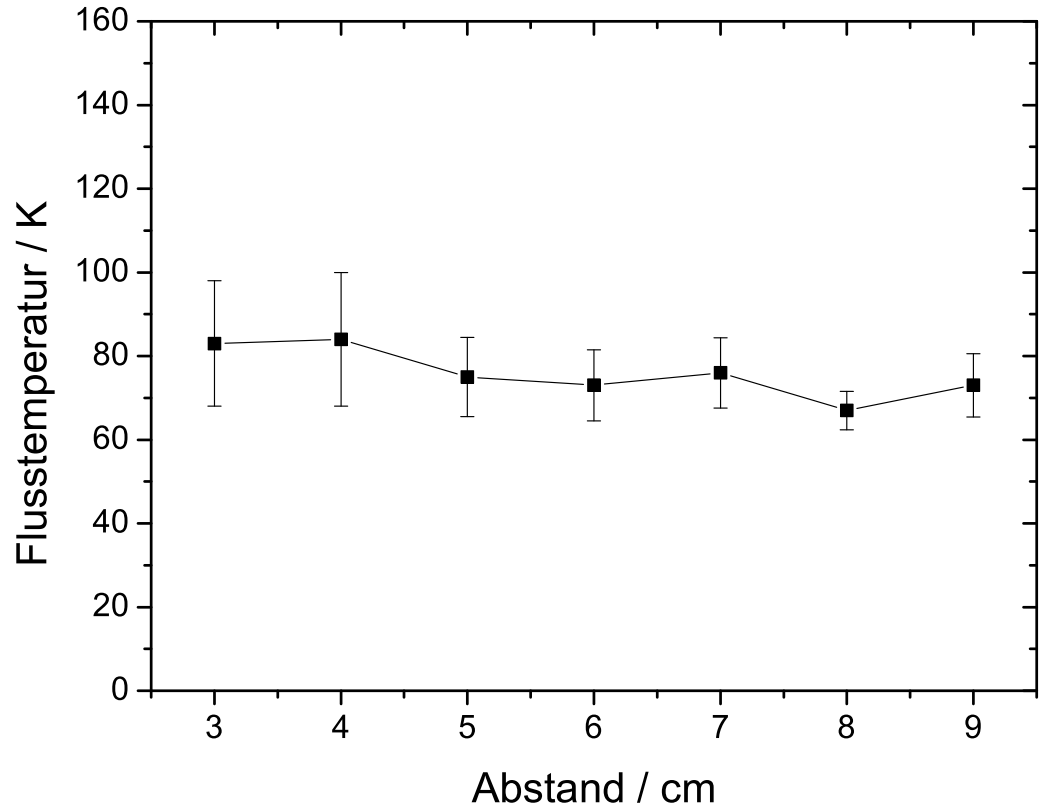

Abbildung 3.13: Temperaturprofil der M408E16-Düse(LIF-Messung)

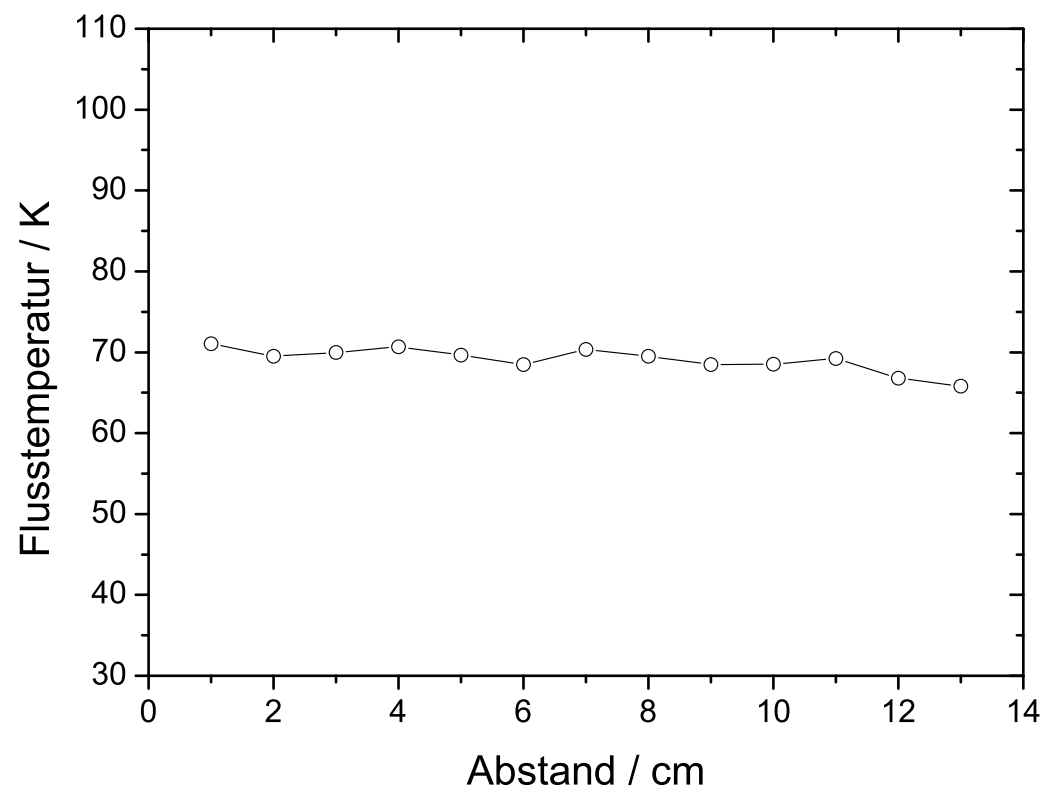

Abbildung 3.14: Temperaturprofil der M408E16-Düse (dynamische Druckmessung) 
des Aufpralldrucks $p_{\mathrm{i}}$ und des Druckes in der Stagnationsregion $p_{0}$ in Abhängigkeit des Abstandes von der Düsenöffnung durchgeführt. Dazu wurde der Druckaufnehmer für $p_{\text {i }}$ fest installiert und die Halterung mit der Lavaldüse mittels eines Motors in axialer Richtung verschoben. Ergebnisse dieser Messungen sind in Abb. 3.14 und 3.15 gezeigt.

Ein Vergleich der Flusstemperaturbestimmung zeigt, das die Ergebnisse aus den rotationsaufgelösten LIF-Spektren systematisch höhere Temperaturen ergeben als die aus den dynamischen Druckmessungen erhaltenen Werte. Da die Daten aus den LIF-Messungen einen größeren Fehler aufweisen, werden in dieser Arbeit nur die Flusstemperaturen aus den letzteren Messungen angegeben. Die Dichte im Fluss, die wichtig und nötig für die Berechnung der Konzentration der Reaktanden ist, kann nur über diese Methode bestimmt werden. Weiterhin war es auch möglich, den Druckaufnehmer für den Aufpralldruck $p_{\text {i }}$ quer zum Strahl auf eine beliebige Position zu verschieben, so dass ein Querschnittsprofil des Düsenstrahls aufgenommen werden konnte, wobei der Aufpralldruck $p_{\mathrm{i}}$ in der Grenzschicht, die den isentropen Flusskern umgibt, nicht in einen statischen Druck $p$ umgerechnet werden konnte, da in der Grenzschicht Gleichung 3.27 nicht gilt.

In Tabelle 3.1 sind alle in Göttingen gefertigten und getesten Düsen mit ihren berechneten und experimentell bestimmten Bedingungen aufgeführt.

Die Düsenbezeichnung in Tab. 3.1 erklärt sich wie folgt: die ersten zwei Ziffern beziehen sich auf die Machzahl, die weiteren geben die Gesamtteilchenzahldichte im Strahl an. Somit hat die M351E17-Düse eine Machzahl von 3.5 und eine Flussdichte von $1 \cdot 10^{17} \mathrm{~cm}^{-3}$.

Die Kombination von axialer und radialer Verschiebung ermöglicht es also, die Flussbedingungen an jeder Stelle im Strahl zu bestimmen, es ergibt sich ein dreidimensionales Bild des Strahls, die Abhängigkeit von $p_{\text {i }}$ von der axialen und radialen Position für die M48E16-Düse ist in Abb. 3.16 gezeigt.

\begin{tabular}{|c|cc|lc|c|}
\hline Düsenbezeichnung & \multicolumn{2}{|c|}{ Flusstemperatur $[\mathrm{K}]$} & \multicolumn{2}{|c|}{ Flussdichte $\left[10^{17} \mathrm{~cm}^{-3}\right]$} & Machzahl \\
& exp. & ber. & exp. & ber. & \\
\hline \hline M211E17 & 114 & 159 & 1.0 & 1.0 & 2.1 \\
M278E16 & 101 & 122 & 0.5 & 0.8 & 2.7 \\
M351E17 & 84 & 87 & 1.0 & 1.0 & 3.5 \\
M408E16 & 71 & 71 & 0.9 & 0.8 & 4.0 \\
M508E16 & 58 & 50 & 0.5 & 0.8 & 5.0 \\
\hline
\end{tabular}

Tabelle 3.1: verwendete Lavaldüsen 


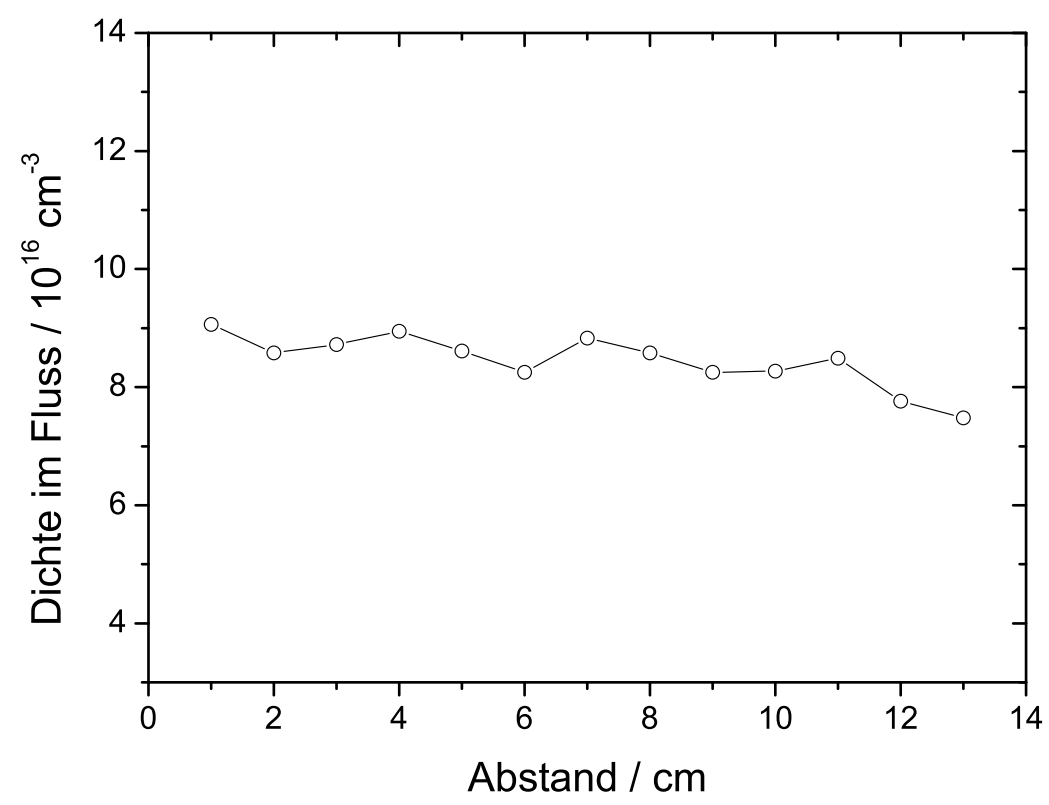

Abbildung 3.15: Dichteprofil der M408E16-Düse (dynamische Druckmessung)

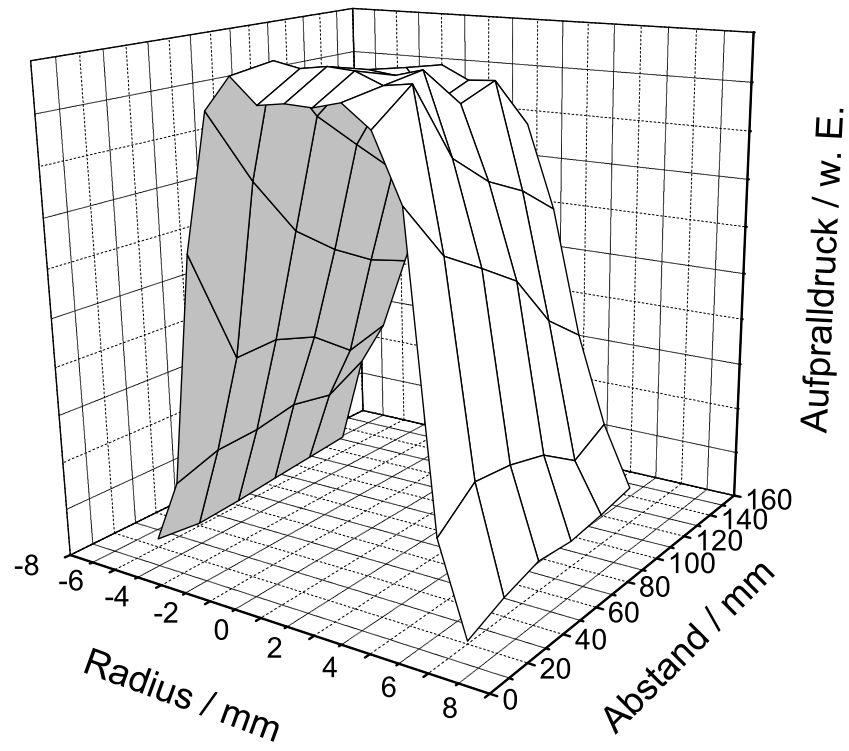

Abbildung 3.16: Dreidimensionales Abbild der Expansion einer M408E16-Düse 



\section{Kapitel 4}

\section{Ergebnisse}

\subsection{Relaxation des OH-Radikals}

Im elektronischen Grundzustand des Hydroxylradikals $\left(X^{2} \Pi_{i}\right)$, dessen Population in den gemessenen LIF-Spektren zeitabhängig beobachtet wurde, findet sowohl Rotations- als auch Schwingungsenergietransfer (RET und VET für Rotational bzw. Vibrational Energy Transfer) statt, da die Hydroxylradikale in den vorliegenden Experimenten in einer nicht-Gleichgewichtsbesetzung präpariert werden. Bei der Photolyse von Wasserstoffperoxid $\left(\mathrm{H}_{2} \mathrm{O}_{2}\right)$ mit einem Excimerlaser (Wellenlänge: $193 \mathrm{~nm}$ ) wird eine gewisse Überschussenergie frei, die zunächst als Translations-, Schwingungs- und Rotationsenergie in den entstandenen OH-Radikalen gespeichert wird. Dann fließst diese Energie aus den Radikalen in das Badgas ab, und zwar mit einer individuellen Zeitkonstante für jeden Prozeß. Da die Fluoreszenz in den vorliegenden Experimenten sowohl durch den (2,1)- als auch durch den (1,0)-Übergang angeregt werden konnte, wurde sowohl der Rotationergietransfer im Schwingungszustand $v^{\prime \prime}=1$ bzw. $v^{\prime \prime}=0$ als auch der Schwingungsenergietransfer aus dem ersten angeregten Schwingungzustand des elektronischen Grundzustand in eine Gleichgewichtsbesetzung zeitlich verfolgt. Die Bedeutung der Messung der Schwingungsrelaxation liegt in der Vergleichbarkeit ihrer Geschwindigkeitskonstanten mit Hochdruckgrenzwerten (unter bestimmten Umständen).

Die Geschwindigkeitskonstante für die Rotationsrelaxation konnte auf zwei unterschiedliche Arten bestimmt werden (Abb. 4.1). Bei der Aufnahme der zeitabhängigen Fluoreszenzsignale im Schwingungsgrundzustand ist vor dem Abfall des LIFSignals, der durch die Reaktion des OH-Radikals mit dem ungesättigten Kohlenwasserstoff zustande kommt, eine schneller Anstieg des LIF-Signals zu beobachten. Dieser Anstieg findet auf einer wesentlich kürzeren Zeitskala als die Reaktion statt und wird als Rotationsrelaxation interpretiert. Die in den kinetischen Experimenten beobachtete Fluoreszenzintensität der $Q_{1}(1)$-Linie repräsentiert also die Besetzung 


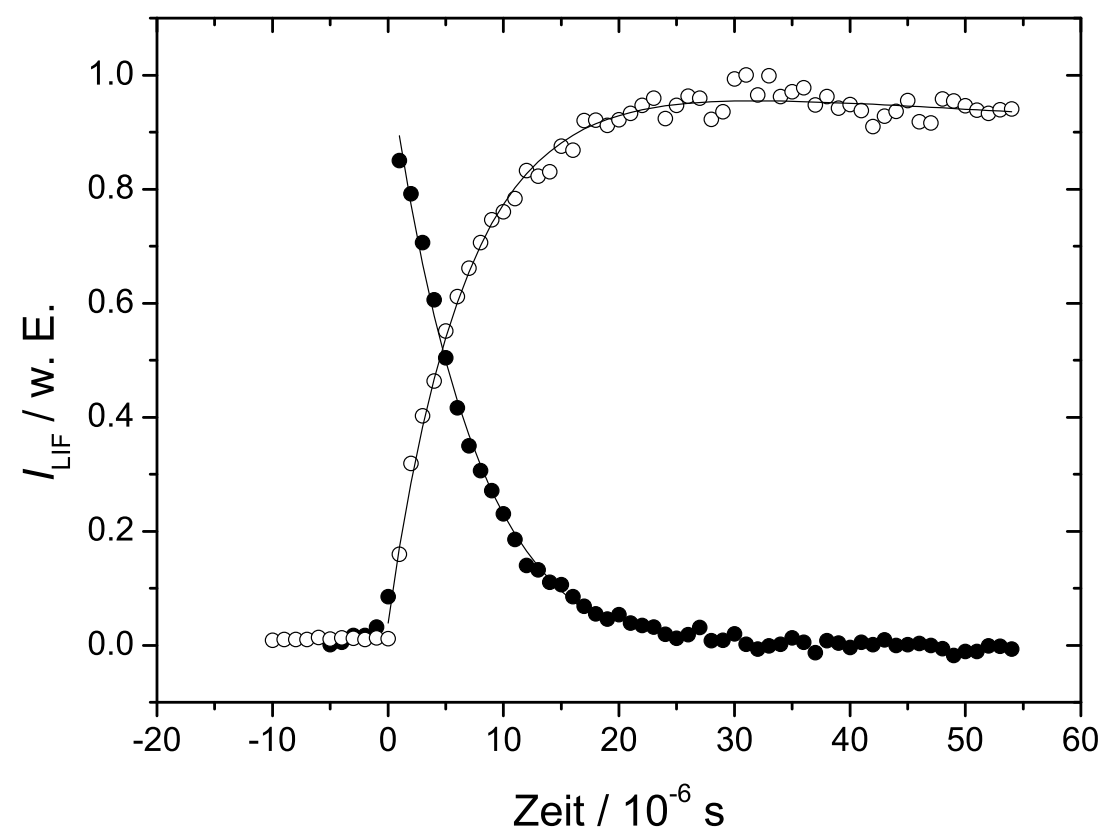

Abbildung 4.1: Rotationsrelaxation des Hydroxylradikals in verschiedenen Quantenzuständen: • $\mathrm{Q}_{1}(12)$-Linie $\circ \mathrm{Q}_{1}(1)$-Linie

der niedrigsten Rotationsniveaus im Schwingungsgrundzustand. Zu Anfang eines jeden kinetischen Experiments ist die Zeitverzögerung zwischen Photolyse- und Fluoreszenzanregungslaser sehr kurz, d. h. die Hydroxylradikale werden direkt nach ihrer Entstehung detektiert, zu diesem Zeitpunkt sind sie rotationsangeregt. Deswegen sind die niedrigen Rotationsniveaus (vgl. $\circ \mathrm{Q}_{1}(1)$-Linie in Abb. 4.1) zu Beginn der Messungen kaum bis gar nicht besetzt, die Population wächst im Laufe der Zeit monoexponentiell auf einen Gleichgewichtswert an. Wird der Probelaser auf eine Linie mit hoher Rotationsquantenzahl abgestimmt (z. B. die $\mathrm{Q}_{1}(12)$-Linie), so ist genau der umgekehrte Fall zu beobachten. Bei der Entstehung der Hydroxylradikale sind hohe Rotationsniveaus besetzt, deren Besetzung schnell monoexponentiell abnimmt, nach ca. $30 \mu$ s sind diese Niveaus nahezu komplett entvölkert ((vgl. • Q ${ }_{1}(12)$-Linie in Abb. 4.1). Werden beide Signale mit einer monoexponentiellen Funktion angepasst (Anstieg bzw. Abfall), so ergeben sich innerhalb der Fehlergrenzen identische Zeitkonstanten.

Bisherige Untersuchungen zu Schwingungsrelaxation im $\left(X^{2} \Pi_{i}, v^{\prime \prime}=1\right)$-Zustand ergeben für in dieser Arbeit vorliegenden Teilchendichten $\left(\rho \approx 10^{17} \mathrm{~cm}^{-3}\right)$ laut 
Ref. [189] Relaxationszeiten von $77\left(\mathrm{O}_{2}\right)$ bzw. $56 \mu \mathrm{s}\left(\mathrm{CO}_{2}\right)$ an, allerdings wurden nur die Stoßgase Sauerstoff und Kohlendioxid bei Raumtemperatur untersucht. Die Schwingungsrelaxation aus dem $\left(X^{2} \Pi_{i}, v^{\prime \prime}=2\right)$-Zustand ist bei gleichen Stoßgasen etwas schneller, für $\mathrm{O}_{2}$ ergeben sich $38 \mu \mathrm{s}$, für $\mathrm{CO}_{2} 15 \mu \mathrm{s}$ [190]. Das in dieser Arbeit verwendete Trägergas Stickstoff erwies sich in Ref. [190] als recht ineffizienter Stoßpartner, es wurde nur eine untere Grenze von $1 \mathrm{~ms}$ für die Relaxationszeit angegeben.

In Ref. [191] findet sich ebenfalls nur eine unterer Grenze für die Schwingungsrelaxation des $\left(X^{2} \Pi_{i}, v^{\prime \prime}=1\right)$-Zustandes in Argon von $20 \mu$ s. Während die Schwingungsrelaxation in $\left(X^{2} \Pi_{i}\right)$ also im Allgemeinen auf einer Zeitskala von 10 bis $100 \mu \mathrm{s}$ stattfindet, vollzieht sich der Rotationsenergietransfer wesentlich schneller, bei Atmosphärendruck zwischen 10 und 100 ns [192]. In einer Untersuchung der Rotationsrelaxation von OH-Radikalen im Schwingungsgrundzustand in Stickstoff wurde eine Geschwindigkeitskonstante von $2.6 \cdot 10^{-11} \mathrm{~cm}^{-3} \mathrm{~s}^{-1}$ bestimmt [193], die über mehrere Rotationszustände gemittelt wurde. Bei der in den vorliegenden Experimenten Teilchendichte von $10^{17} \mathrm{~cm}^{-3}$ ergibt sich also eine Relaxationszeit von $385 \mathrm{~ns}$. Die Schwingungsrelaxation in elektronisch angeregtem $\mathrm{OH}\left(A^{2} \Sigma^{+}\right)$findet auf einer vergleichbaren Zeitskala statt, da sie um einen Faktor 10 bis 100 schneller ist als derselbe Prozess im Grundzustand. Der Unterschied der Schwingungsrelaxationsgeschwindigkeiten lässt sich über den aus dem Hydroxylradikal und dem Badgas gebildeten van-der-Waals-Komplex deuten, dessen Potentialminimum im elektronisch angeregten Zustand $\left(A^{2} \Sigma^{+}\right)$wesentlich ausgeprägter ist als im Grundzustand [194].

Die in unseren Experimenten beobachteten Rotationsrelaxationszeiten lagen sowohl für $v^{\prime \prime}=0$ als auch für $v^{\prime \prime}=1$ in einem Bereich zwischen 500 ns und $5 \mu$ s und sind somit etwas langsamer als die oben angegebenen Werte, was z. B. durch die in der Lavaldüsenexpansion erzeugten, wesentlich tieferen Temperaturen zu erklären ist. Die Geschwindigkeit der Schwingungsrelaxation wurde auf einer Zeitskala von wenigen $100 \mu$ s beobachtet (vgl. Abb. 4.2), was in etwa den bisherigen experimentellen Beobachtungen in der Literatur entspricht. Dabei ist jedoch zu beachten, das der beobachtete Abfall der Fluoreszenzintensität nicht allein durch die Relaxation aus dem angeregten Schwingungszustand zustande kommt, sondern auch durch die Diffusion der erzeugten Hydroxylradikale aus dem Lavaldüsenstrahl, die auch bei den Messungen im Schwingungsgrundzustand beobachtet wurde. 


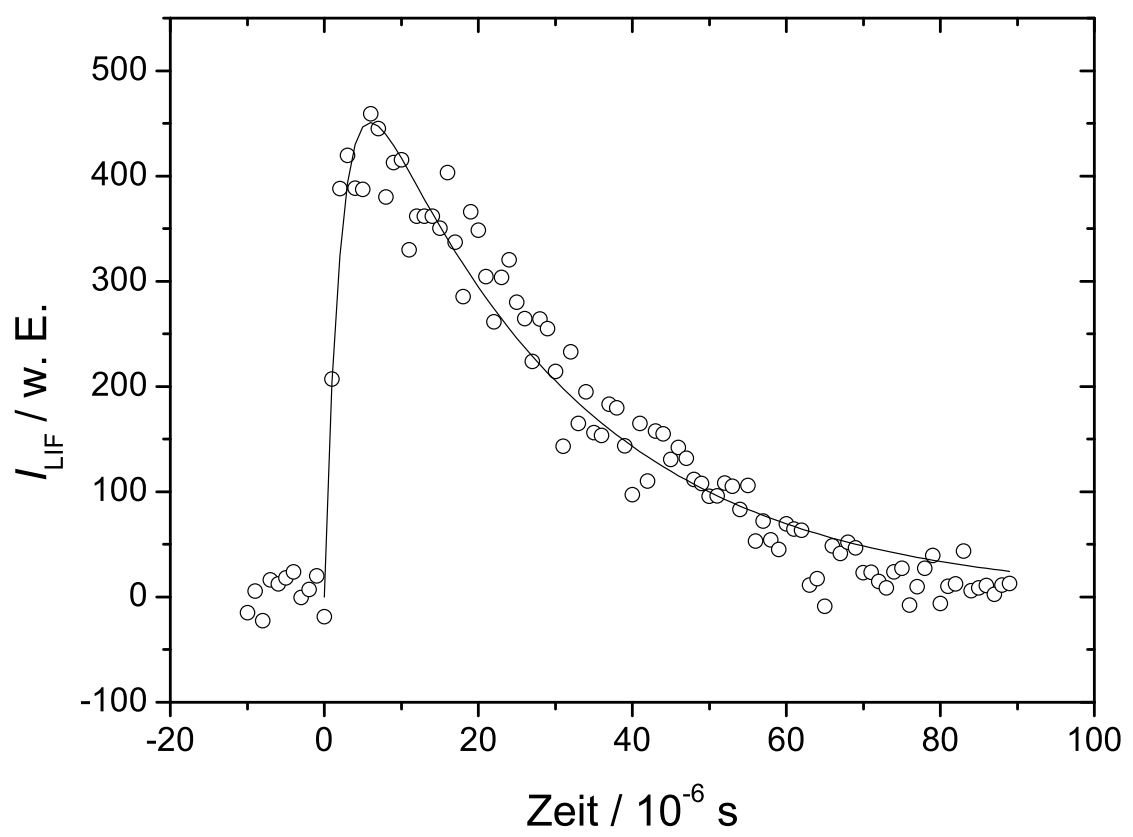

Abbildung 4.2: Schwingungsrelaxation des OH-Radikals (in $\mathrm{C}_{2} \mathrm{H}_{4} / \mathrm{N}_{2}$ ); $T=98 \mathrm{~K}$

\subsection{Reaktionen des OH-Radikals}

Um die Geschwindigkeitskonstante 2. Ordnung für die Reaktionen der ungesättigten Kohlenwasserstoffe mit OH-Radikalen zu erhalten, wurde die zeitabhängige $\mathrm{OH}$ Radikalkonzentration für verschiedene Reaktandenkonzentrationen aufgenommen.

$$
\mathrm{OH}+\mathrm{X} \stackrel{k_{2}^{\prime}}{\longrightarrow} \mathrm{OHX}
$$

X steht hier für einen ungesättigten Kohlenwasserstoff. Aus der Steigung einer Auftragung der Geschwindigkeitskonstanten pseudo-erster Ordnung (Abb. 4.3 und 4.4) gegen die Konzentration des Reaktanden konnte dann die Geschwindigkeitskonstante zweiter Ordnung abgelesen werden (Abb. 4.5 und 4.6).

Für die Reaktionen von Ethin und Ethen mit $\mathrm{OH}$ wurde die zeitliche Entwicklung der OH-Radikalpopulation jeweils sowohl im Schwingungsgrundzustand des elektronischen Grundzustandes $\left(X^{2} \Pi_{i}, v^{\prime \prime}=0\right)$ als auch im ersten angeregten Schwingungszustand $\left(X^{2} \Pi_{i}, v^{\prime \prime}=1\right)$ aufgenommen. Aus beiden Untersuchungen ergeben sich zunächst Geschwindigkeitskonstanten zweiter Ordnung, die aufgrund der 


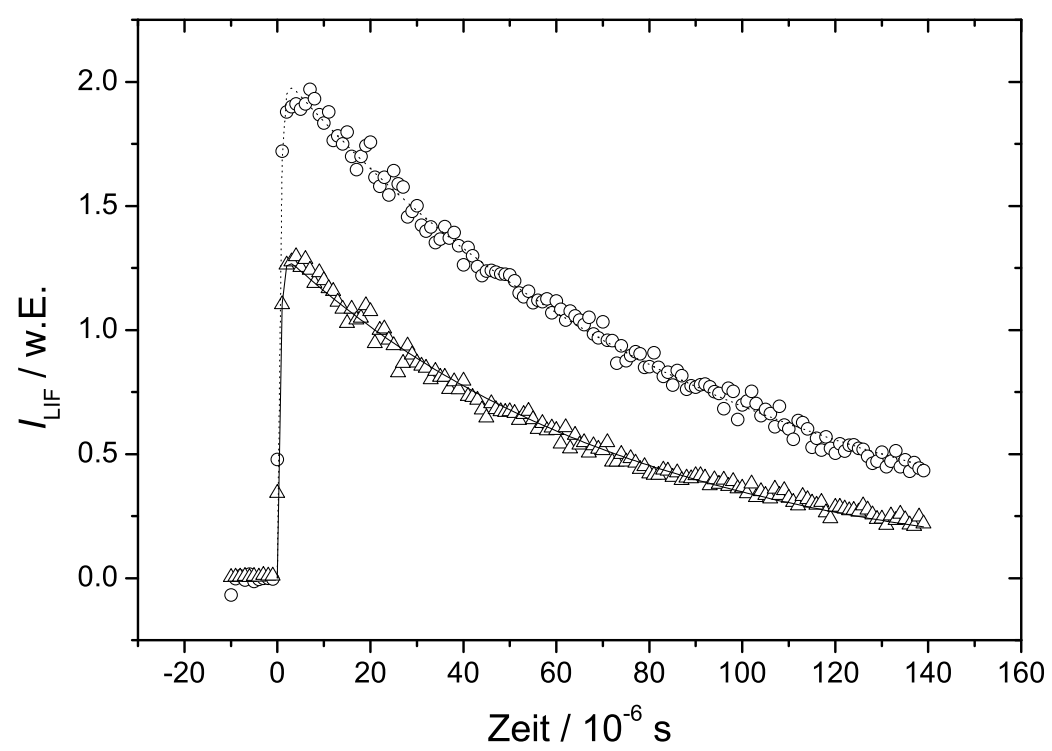

Abbildung 4.3: Kinetiken pseudo-erster Ordnung bei verschiedener Reaktandenkonzentration $\left(\mathrm{OH}\left(v^{\prime \prime}=0\right)+\mathrm{C}_{2} \mathrm{H}_{2}, T=70 \mathrm{~K}\right.$, Konzentration $\left.3.6: 1\right)$

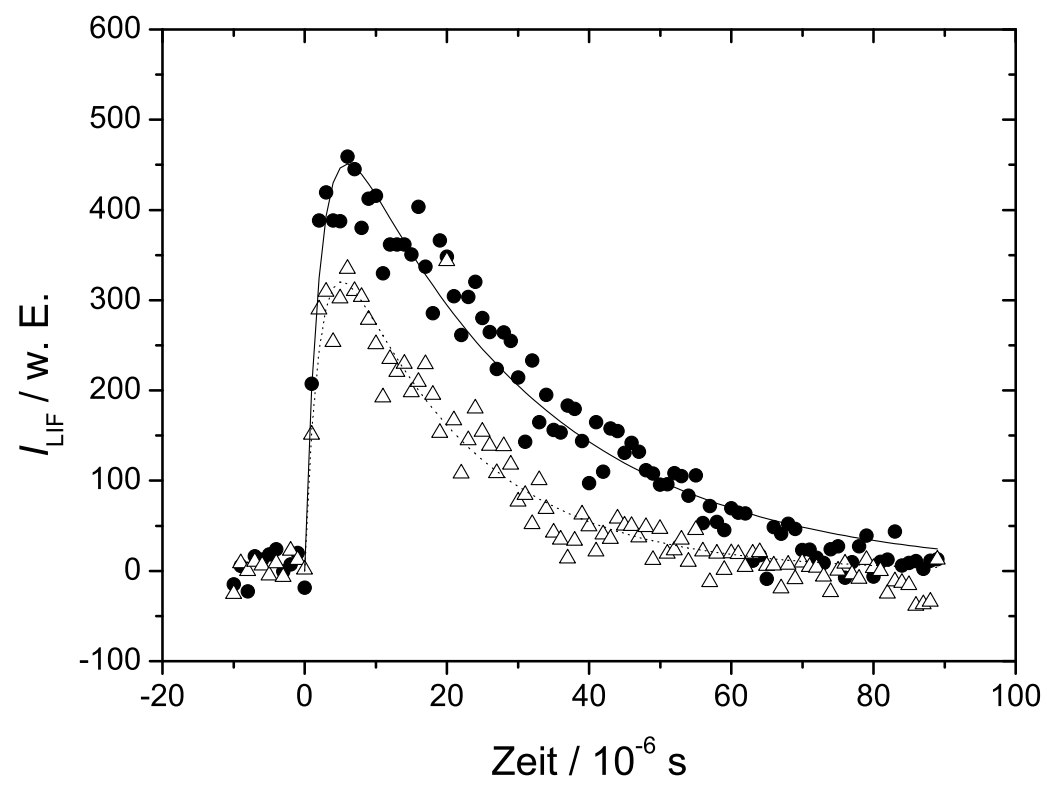

Abbildung 4.4: Kinetiken pseudo-erster Ordnung bei verschiedener Reaktandenkonzentration $\left(\mathrm{OH}\left(v^{\prime \prime}=1\right)+\mathrm{C}_{2} \mathrm{H}_{4}, T=98 \mathrm{~K}\right.$, Konzentration $\left.7.5: 1\right)$ 


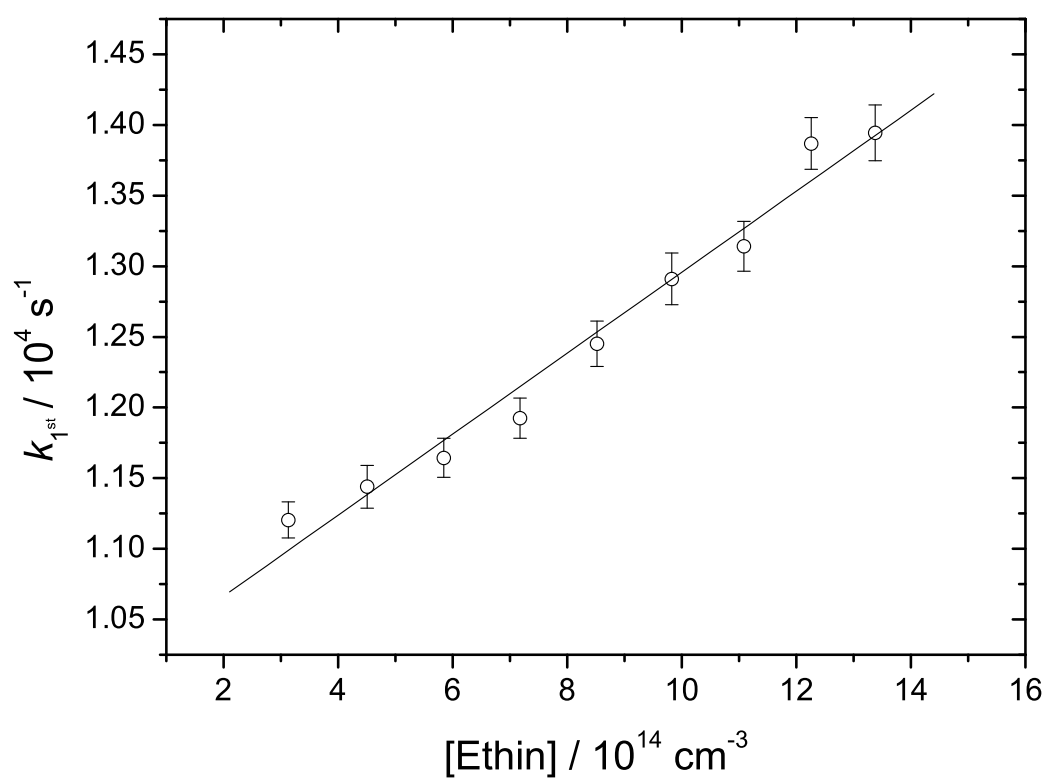

Abbildung 4.5: Geschwindigkeitskonstanten pseudo-erster Ordnung in Abhängigkeit der Reaktandenkonzentration $\left(\mathrm{OH}\left(v^{\prime \prime}=0\right)+\mathrm{C}_{2} \mathrm{H}_{2}, T=70 \mathrm{~K}\right)$

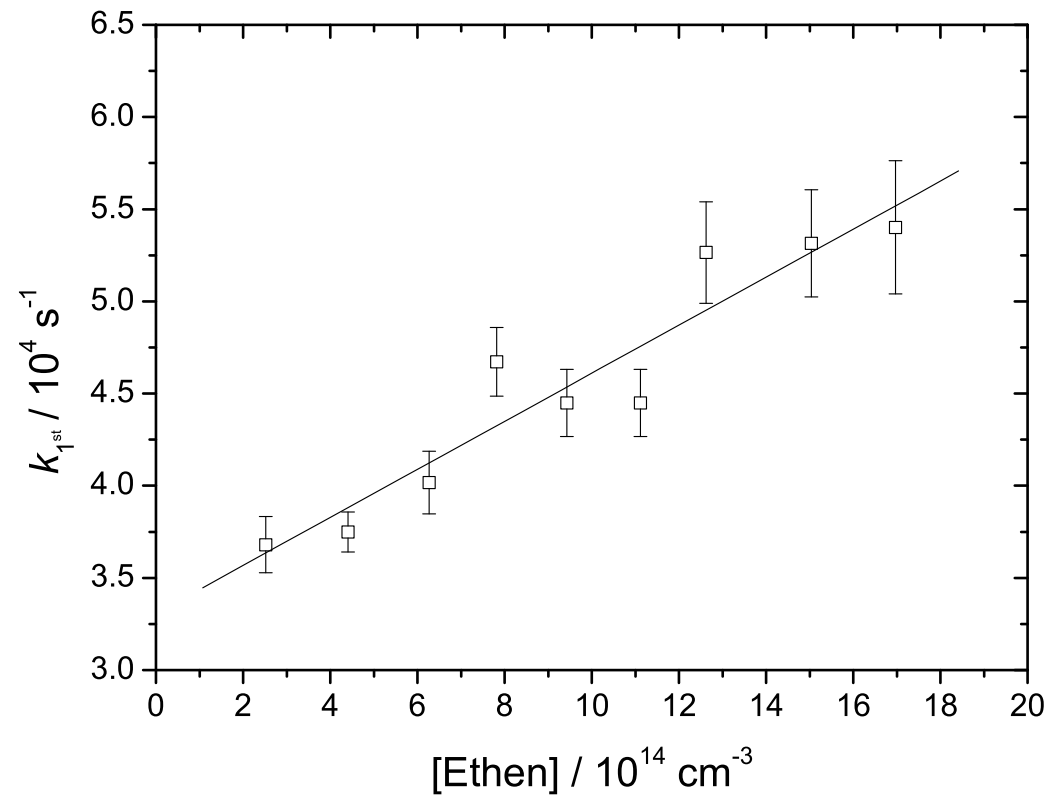

Abbildung 4.6: Geschwindigkeitskonstanten pseudo-erster Ordnung in Abhängigkeit der Reaktandenkonzentration $\left(\mathrm{OH}\left(v^{\prime \prime}=1\right)+\mathrm{C}_{2} \mathrm{H}_{4}, T=98 \mathrm{~K}\right)$ 
Druckabhängigkeit der untersuchten Reaktionen weitere Betrachtung benötigt. Die Druckabhängigkeit termolekularer Reaktionen wird häufig mit dem Konzept der Fall-Off-Kurven beschrieben, das ursprünglich für unimolekulare Reaktionen vorgesehen war (vgl. Abschnitt 2.1). Wie in Abschnitt 2.1.1 ausführlich beschrieben, führt die Reaktion eines Moleküls A zu einem Produkt P unter Teilnahme des Badgases M zu einer Druckabhängigkeit der Reaktionsgeschwindigkeitskonstanten $k_{\text {uni }}$. Es lassen sich zwei Grenzwerte betrachten, eine druckabhängige Niederdruckgeschwindigkeitskonstante $\left(k_{0}=k_{1} \cdot[\mathrm{M}]\right)$ und eine druckunabhängige Hochdruckgeschwindig-

keitskonstante $\left(k_{\infty}=\frac{k_{1} k_{2}}{k_{-1}}\right)$, der Übergang zwischen den beiden Grenzwerten wird Fall-Off-Bereich genannt. Ein Problem jeder druckabhängigen kinetischen Messung ist nun, in welchen dieser Bereiche ist die bestimmte Geschwindigkeitskonstante einzuordnen? Eine pauschale Aussage über die Drücke, die den verschiedenen Bereichen zugeordnet werden können, ist nicht möglich. Bei welchen Drücken und in welcher Form der Übergang in den Hochdruckbereich stattfindet, hängt in entscheidender Weise von den Moleküleigenschaften und der Temperatur ab. So ist der Hochdruckbereich bei $300 \mathrm{~K}$ für viele atmosphärenchemisch relevante Reaktionen bereits bei Atmosphärendruck erreicht, dahingegen befinden sich z. B. die bisher mit der in Göttingen aufgebauten CRESU-Apparatur untersuchten Reaktionen des Hydroxylradikals mit Propen und Isopren [90] bei Raumtemperatur und darunter bereits bei wenigen Millibar in der druckunabhängigen Region. Zur Temperaturabhängikeit des Erreichens des Hochdruckgrenzwertes lassen sich folgende Überlegungen anstellen. Bei der Addition des Hydroxylradikals an einen ungesättigten Kohlenwasserstoff wird im Allgemeinen ein energiereiches Addukt gebildet, welches durch Stöße mit dem Badgas M stabilisiert wird. Mit sinkender Temperatur wird das Addukt mit geringerer Überschussenergie gebildet, deshalb werden weniger Stöße zur Stabilisierung des Adduktes benötigt, bevor es zu den Edukten zurückdissoziieren kann. Deswegen wird der Fall-Off-Bereich mit sinkender Temperatur zu kleineren Drücken verschoben. In den folgenden zwei Abschnitten werden nun die experimentellen Ergebnisse präsentiert und erörtert, wie die gemessenen Geschwindigkeitskonstanten für OH-Radikale im Schwingungsgrundzustand $\left(v^{\prime \prime}=0\right)$ und im ersten schwingungsangeregten Zustand $\left(v^{\prime \prime}=1\right)$ einzuordnen sind.

\subsubsection{Ethin $+\mathrm{OH}$}

In Tab. 4.1 sind die in dieser Arbeit experimentell bestimmten Geschwindigkeitskonstanten in Abhängigkeit der Temperatur angegeben und in Abb. 4.7 aufgetragen. Die gemessenen Geschwindigkeitskonstanten liegen alle in einem Bereich von $2-3 \cdot 10^{-12} \mathrm{~cm}^{-3}$, diese Reaktion kann also als relativ langsam im Vergleich zu Re- 


\begin{tabular}{|l|c|c|cc|}
\hline Düse & $T[\mathrm{~K}]$ & $\rho\left[10^{17} \mathrm{~cm}^{-3}\right]$ & \multicolumn{2}{|c|}{$k_{2 . \text { Ord. }}\left[10^{-12} \mathrm{~cm}^{3} \cdot \mathrm{s}^{-1}\right]$} \\
& & & $v^{\prime \prime}=1$ \\
\hline \hline- & 298 & 3.8 & $(0.41 \pm 0.08)$ & $(2.22 \pm 0.69)$ \\
M211E17 & 114 & 1.0 & $(0.77 \pm 0.15)$ & $(2.69 \pm 1.05)$ \\
M278E16 & 101 & 0.52 & $(1.60 \pm 0.32)$ & $(2.28 \pm 0.92)$ \\
M351E17 & 84 & 0.97 & $(1.50 \pm 0.30)$ & $(2.49 \pm 1.13)$ \\
M408E16 & 71 & 0.90 & $(3.91 \pm 0.79)$ & $(3.04 \pm 0.74)$ \\
M508E16 & 58 & 0.51 & $(2.55 \pm 0.51)$ & - \\
\hline
\end{tabular}

Tabelle 4.1: Mit Hilfe der Göttinger CRESU-Apparatur gemessene Geschwindigkeitskonstanten für die Reaktion des $\mathrm{OH}$ mit Ethin

aktionen des Hydroxylradikals mit anderen ungesättigten Kohlewasserstoffen angesehen werden, die sich in einem Bereich zwischen $10^{-11}$ und $10^{-10} \mathrm{~cm}^{-3}$ bewegen. Damit nimmt diese Reaktion eine Sonderstellung ein, die in Abschnitt 5 noch näher diskutiert werden soll. Zunächst ergibt sich die Frage, in welchem Bereich (Niederdruck, Hochdruck oder Fall-Off) sich die gemessenen Geschwindigkeitskonstanten befinden, da in dieser Arbeit nicht die Druckabhängigkeit über weite Bereiche untersucht worden ist. Die bestimmte Geschwindigkeitskonstante für die Reaktion eines OH-Radikals im Schwingungsgrundzustand $\left(v^{\prime \prime}=0\right)$ mit Ethin bei Raumtemperatur (o) liegt im Vergleich zu den von Hippler et al. [30] bestimmten Hochdruckgeschwindigkeitskonstanten ( $\mathbf{\Delta})$ um einen Faktor fünf niedriger.

Die Reaktion befindet sich also sehr wahrscheinlich bei $298 \mathrm{~K}$ unter den vorliegenden Drücken (ca. 10 mbar) keines Falls im Hochdruckbereich. Vielmehr zeigt die Fall-OffKurve bei Raumtemperatur [30], dass sich unser gemessener Wert im ansteigenden Teil der Fall-Off-Kurve, also im Niederdruckbereich oder im Fall-Off-Bereich befindet. Wird die gemessene Geschwindigkeitskonstante 2. Ordnung auf die vorliegende Teilchenzahldichte bezogen, so ergibt sich ein Wert von $1.1 \cdot 10^{-30} \mathrm{~cm}^{6} \cdot \mathrm{s}^{-1}$, der unterhalb des empfohlenen Niederdruckgrenzwertes von $5.0 \cdot 10^{-30} \mathrm{~cm}^{6} \cdot \mathrm{s}^{-1}$ [68] liegt. Dies deutet darauf hin, dass sich die bestimmte Geschwindigkeitskonstante nicht im Niederdruck- sondern im Fall-Off-Bereich befindet. Somit ist die Bestimmung von Niederdruckgeschwindigkeitkonstanten ohne die Variation des Druckes, die sich für CRESU-Experimente nur innerhalb einer Größenordnung bewegt, nicht mit der Erstellung einer vollständigen Fall-Off-Kurve vergleichbar.

Dahingegen zeigt der Vergleich der hier bestimmten $\left(v^{\prime \prime}=1\right)$-Geschwindigkeitskonstante bei Raumtemperatur $\left(2.2 \cdot 10^{-12} \mathrm{~cm}^{3} \cdot \mathrm{s}^{-1}\right)(\boldsymbol{\square})$ eine sehr gute Übereinstimmung mit der von Hippler et al. angegebenen Hochdruckgeschwindigkeitskonstanten $\left(2.0 \cdot 10^{-12} \mathrm{~cm}^{3} \cdot \mathrm{s}^{-1}\right)$. Somit scheint das Konzept, Hochdruckgrenzwerte aus 


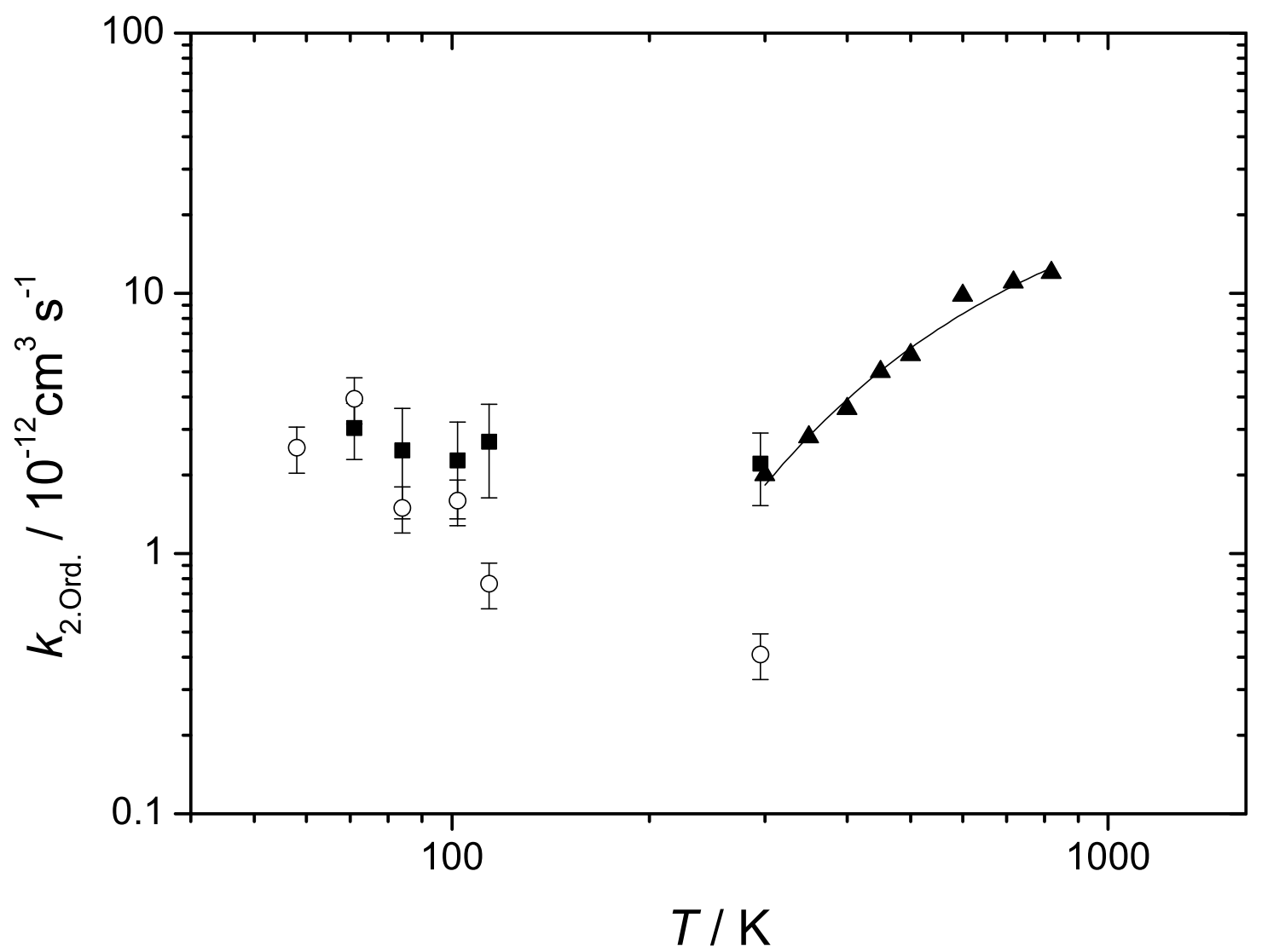

Abbildung 4.7: Doppelt logarithmische Auftragung der Geschwindigkeitskonstanten 2. Ordnung für $\left(\mathrm{OH}+\mathrm{C}_{2} \mathrm{H}_{2}\right)$; o v" $=0$, $\mathbf{v} "=1$, $\boldsymbol{\Delta}$ Hippler et al. $k_{\infty}[30]$

Messungen der Schwingungsrelaxation zu erhalten, für diese Reaktion aufzugehen. Dieses Konzept erweist sich in diesem Fall als sehr nützlich, denn die von Hippler et al. bei 80 bar bestimmte Geschwindigkeitskonstante befindet sich offensichtlich noch nicht vollständig im Hochdruckbereich. Dabei ist allerdings zu beachten, dass die Abschätzung des Hochdruckgrenzwertes über die Schwingundrelaxation immer einen oberen Grenzwert liefert. Der Vergleich der in der CRESU-Apparatur in Göttingen bestimmten Geschwindigkeitskonstanten für $\mathrm{OH}\left(v^{\prime \prime}=0\right.$ und $\left.v^{\prime \prime}=1\right)$ in Abb. 4.7 zeigt, dass sich die Messungen im Schwingungsgrundzustand des Hydroxylradikals (o) mit sinkender Temperatur den Werten der $v^{\prime \prime}=1$-Messungen annähern. Ab einer Temperatur von ca. $80 \mathrm{~K}$ sind die Geschwindigkeitskonstanten innerhalb der Fehlergrenzen nicht mehr voneinander zu unterscheiden. Bei Gültigkeit der Annahme, dass sich die $v^{\prime \prime}=1$-Messungen mit den Hochdruckgeschwindig- 
keitskonstanten vergleichen lassen, kann also davon ausgegangen werden, dass sich die Reaktion $\left(\mathrm{C}_{2} \mathrm{H}_{2}+\mathrm{OH}\right)$ ab einer Temperatur von $80 \mathrm{~K}$ und darunter bei den in dieser Arbeit verwendeten Drücken von wenigen Millibar im Hochdruckbereich befindet. Der Effekt, dass sich das Erreichen des Hochdruckbereiches mit fallender Temperatur zu niedrigeren Drücken verschiebt, wurde bereits im vorhergehenden Abschnitt erläutert und ist durch den Verlauf der Geschwindigkeitskonstanten in Abb. 4.7 bestätigt, ein analoges Bild ergeben die temperaturabhängigen Fall-OffKurven in der Arbeit von Michael et al. [56] im Bereich von 228 bis $413 \mathrm{~K}$. Aufgrund der nicht vorhandenen druckabhängigen Bestimmung der Niederdruckgeschwindigkeitskonstanten und der offensichtlich hier gültigen Abschätzung der Hochdruckgeschwindigkeitskonstanten über die Schwingungsrelaxation werden in Abschnitt 5 nur die Ergebnisse der $v^{\prime \prime}=1$-Messungen betrachtet und im Folgenden als Hochdruckgeschwindigkeitskonstanten diskutiert. Die bestimmten Hochdruckgeschwindigkeitskonstanten zeigen eine leicht negative Temperaturabhängigkeit, der Verlauf der Werte von Hippler et al. impliziert ein Minimum der Geschwindigkeitskonstanten in einem Bereich um $200 \mathrm{~K}$.

\subsubsection{Ethen $+\mathrm{OH}$}

Die in Tabelle 4.2 angegebenen temperaturabhängigen Geschwindigkeitskonstanten für die Reaktion $\mathrm{C}_{2} \mathrm{H}_{4}+\mathrm{OH}$ sind in Abb. 4.8 aufgetragen.

Die bestimmten Geschwindigkeitskonstanten sind im Vergleich zur analogen Reaktion mit Ethin um fast eine Größenordnung größer, zeigen aber ein ähnliches Verhalten. Die bei $298 \mathrm{~K}$ gemessene Geschwindigkeitskonstante $\left(v^{\prime \prime}=0\right)$ liegt einen

\begin{tabular}{|c|c|c|c|c|}
\hline Düse & $T[\mathrm{~K}]$ & $\rho\left[10^{17} \mathrm{~cm}^{-3}\right]$ & \multicolumn{2}{|c|}{$k_{2 . \text { Ord. }}\left[10^{-12} \mathrm{~cm}^{3} \cdot \mathrm{s}^{-1}\right]$} \\
\hline - & 298 & 2.7 & $(4.2 \pm 0.8)$ & $\overline{(9.3 \pm 1.9)}$ \\
\hline M21 & 134 & 1.3 & $(10.3 \pm 2.0)$ & - \\
\hline M211E17 & 113 & 1.0 & $(15.9 \pm 3.0)$ & $(14.9 \pm 3.0)$ \\
\hline M278E16 & 98 & 0.55 & $(8.5 \pm 1.3)$ & $(13.1 \pm 2.1)$ \\
\hline M351 & 84 & 0.97 & $(12.0 \pm 2.2)$ & $(10.7 \pm 3.4)$ \\
\hline M40 & 72 & 0.93 & $(12.6 \pm 2.4)$ & $(25.0 \pm 5.2)$ \\
\hline M508E16 & 62 & 0.60 & $(16.1 \pm 3.1)$ & - \\
\hline
\end{tabular}

Tabelle 4.2: Mit Hilfe der Göttinger CRESU-Apparatur gemessene Geschwindigkeitskonstanten für die Reaktion des $\mathrm{OH}$ mit Ethen 


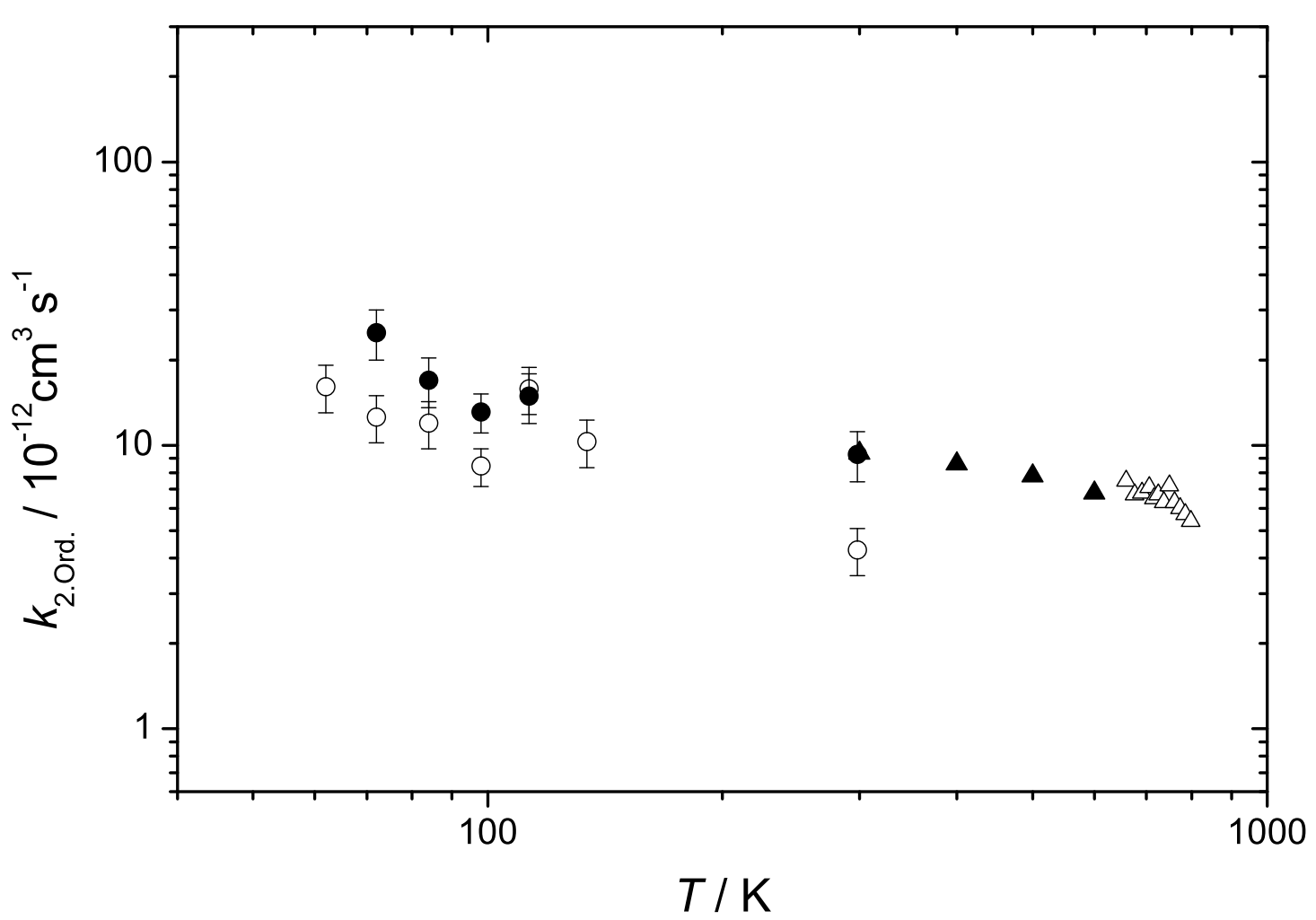

Abbildung 4.8: Doppelt logarithmische Auftragung der Geschwindigkeitskonstanten 2. Ordnung für $\left(\mathrm{OH}+\mathrm{C}_{2} \mathrm{H}_{4}\right)$; $\circ v^{\prime \prime}=0, \bullet v^{\prime \prime}=1, \boldsymbol{\Lambda} 40 \mathrm{bar}, \triangle$ 140 bar ( beide Hippler et al. [30])

Faktor vier niedriger als der Hochdruckgrenzwert von Hippler et al. [30] die Reaktion befindet sich also bei $298 \mathrm{~K}$ und ca. 10 mbar ebenfalls nicht im Hochdruckbereich. Die Umrechnung dieser Geschwindigkeitskonstanten zweiter Ordnung in einen Niederdruckgrenzwert über die vorliegende Teilchendichte ergibt eine Konstante $k_{0}$ von $1.6 \cdot 10^{-29} \mathrm{~cm}^{6} \cdot \mathrm{s}^{-1}$, die deutlich unterhalb des empfohlenen Werts von $6 \cdot 10^{-29} \mathrm{~cm}^{6} \cdot \mathrm{s}^{-1}$ liegt [68].

Die bestimmte Geschwindigkeitskonstante befindet sich also eher im Fall-Off- als im Niederdruckbereich. Somit wird auch hier auf eine weitere Diskussion der für Hydroxylradikale im Schwingungsgrundzustand $\left(v^{\prime \prime}=0\right)$ bestimmten Geschwindigkeitskonstanten verzichtet.

Eine sehr gute Übereinstimmung konnte wieder zwischen der bestimmten Schwingungsrelaxationsgeschwindigkeitskonstanten und den Werten von Hippler et al. [30] für $k_{\infty}$ erzielt werden, die sich laut eigenen Angaben aber noch nicht ganz im Hochdruckbereich befinden. Die Messung der Rekombination über die Schwingungsrela- 
xation ist also auch hier möglich. Eine Konvergenz der beiden gemessenen Geschwindigkeitskonstanten $\left(\mathrm{OH}\left(v^{\prime \prime}=0\right.\right.$ und $\left.\left.v^{\prime \prime}=1\right)\right)$ ist auch in Abb. $4.8 \mathrm{zu}$ erkennen, jedoch scheinen die Werte schon bei höheren Temperaturen übereinzustimmen. Dies deutet darauf hin, dass im Vergleich zur Reaktion mit Ethin die Reaktion von Ethen mit Hydroxylradikalen schon bei kleineren Drücken den Hochdruckbereich erreicht. Ein Vergleich der Fall-Off-Kurven der beiden betrachteten Reaktionen in Ref. [30] gibt das Erreichen des Hochdruckbereiches für die Reaktion des $\mathrm{OH}$ mit $\mathrm{C}_{2} \mathrm{H}_{2}$ bei Raumtemperatur bei ca. 100 bar an, die analoge Reaktion des $\mathrm{C}_{2} \mathrm{H}_{4}$ scheint schon bei 60 bar druckunabhängig zu sein, was die in dieser Arbeit gemachten Beobachtungen bestätigt. Auch für diese Reaktion werden in Abschnitt 5 nur die durch die Schwingungsrelaxation abgeschätzten Hochdruckgeschwindigkeitskonstanten diskutiert.

\subsubsection{Propen $+\mathrm{OH}$}

Die in Tabelle 4.3 angegebenen temperaturabhängigen Geschwindigkeitskonstanten für die Reaktion des Propen mit $\mathrm{OH}$ sind in Abb. 4.9 aufgetragen.

Die in Tab. 4.3 aufgeführten Geschwindigkeitskonstanten wurden unter Beobachtung der Hydroxylradikale im Schwingungsgrundzustand $\left(v^{\prime \prime}=0\right)$ bestimmt. Eine Druckabhängigkeit der Reaktion des Hydroxylradikals mit Propen ist nur bei sehr kleinen Drücken bekannt [28]. So befindet sich diese Reaktion bei Raumtemperatur schon bei ca. 20 mbar im Hochdruckbereich. Unter der Beachtung der Tatsache, dass der Hochdruckgrenzwert bei kleineren Temperaturen schon bei kleineren Drücken erreicht wird, erübrigt sich eine Abschätzung der Hochdruckgrenzwerte über die Beobachtung schwingungsangeregter OH-Radikale $\left(v^{\prime \prime}=1\right)$ in diesem Fall.

\begin{tabular}{|c|c|}
\hline Temperatur $[\mathrm{K}]$ & $k\left[10^{-12} \mathrm{~cm}^{3} \cdot \mathrm{s}^{-1}\right]$ \\
\hline \hline 58 & $57 \pm 9$ \\
71 & $83 \pm 12$ \\
84 & $65 \pm 10$ \\
102 & $73 \pm 11$ \\
114 & $66 \pm 10$ \\
293 & $25 \pm 4$ \\
\hline
\end{tabular}

Tabelle 4.3: Mit Hilfe der Göttinger CRESU-Apparatur gemessene Geschwindigkeitskonstanten für die Reaktion des $\mathrm{OH}$ mit Propen (o) 


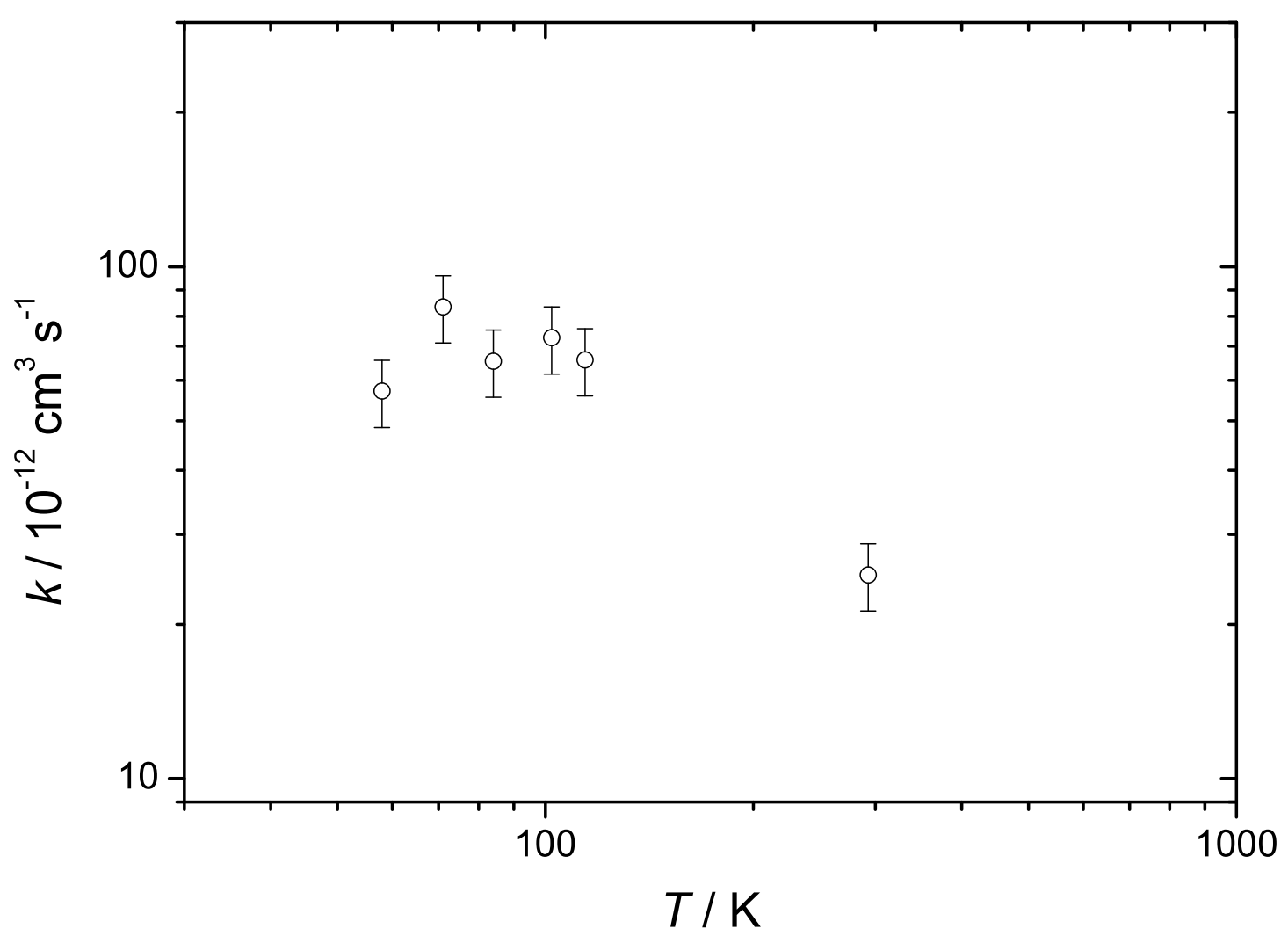

Abbildung 4.9: Auftragung der in dieser Arbeit bestimmten Geschwindigkeitskonstanten für $\mathrm{OH}+$ Propen

\subsubsection{Isopren+OH}

Die in Tabelle 4.4 angegebenen temperaturabhängigen Geschwindigkeitskonstanten für die Reaktion $\mathrm{OH}+$ Isopren sind in Abb. 4.10 aufgetragen.

Die Reaktion des $\mathrm{OH}$ mit Isopren wurde wie die entsprechende Reaktion des Propen ausschlieflich unter Beobachtung der OH-Radikale im Schwingungsgrundzustand $\left(v^{\prime \prime}=0\right)$ untersucht. Die bestimmten Geschwindigkeitskonstanten sollten dennoch druckunabhängig sein, da sich die betrachtete Reaktion bei Raumtemperatur ab einem Druck von ca. 10 mbar im Hochdruckbereich befindet [31]. 


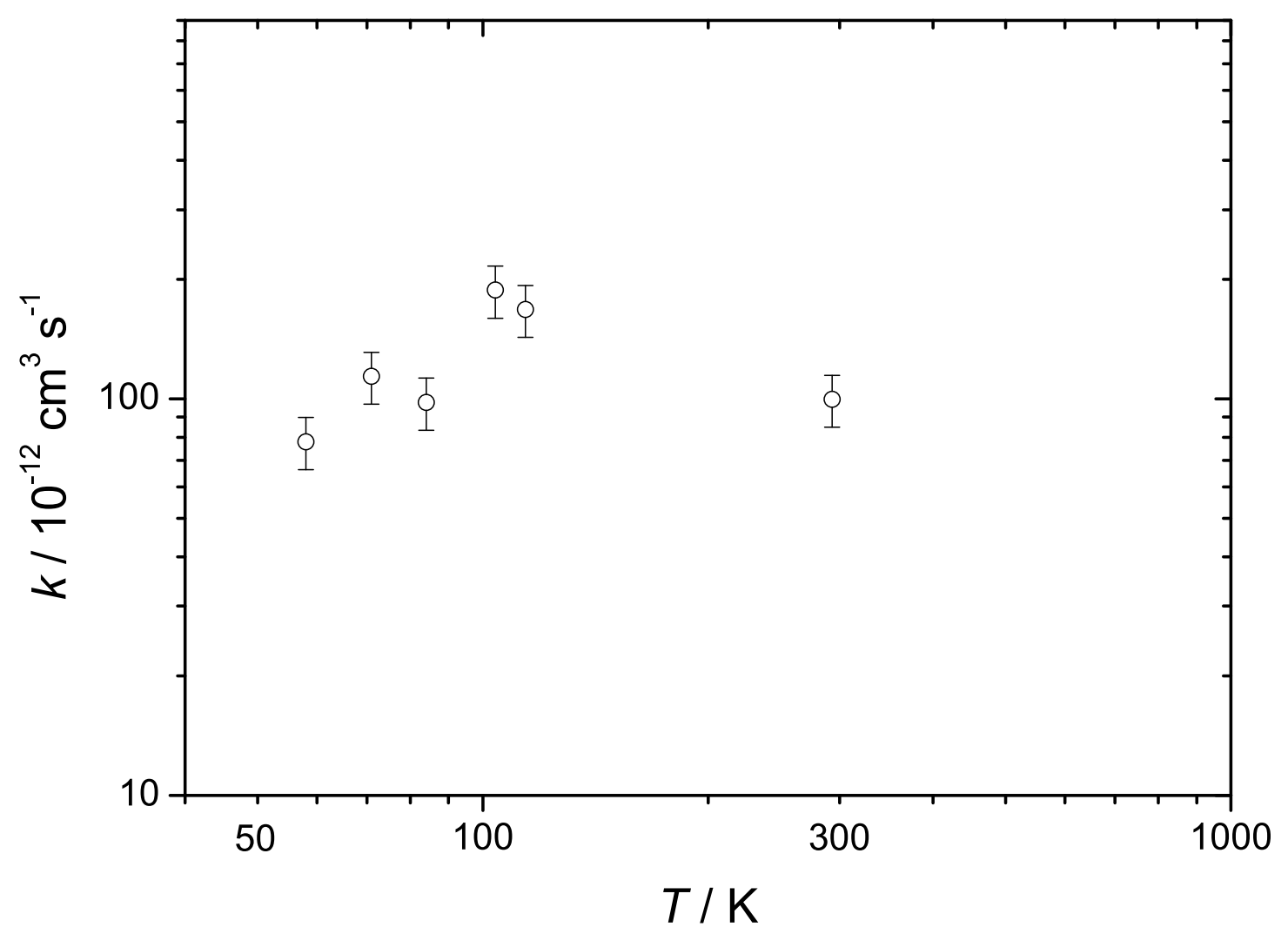

Abbildung 4.10: Auftragung der in dieser Arbeit bestimmten Geschwindigkeitskonstanten für $\mathrm{OH}+$ Isopren

\begin{tabular}{|c|c|}
\hline Temperatur $[\mathrm{K}]$ & $k\left[10^{-12} \mathrm{~cm}^{3} \cdot \mathrm{s}^{-1}\right]$ \\
\hline \hline 58 & $78.0 \pm 12$ \\
71 & $114 \pm 17$ \\
84 & $98.0 \pm 15$ \\
104 & $188 \pm 28$ \\
114 & $168 \pm 25$ \\
293 & $99.7 \pm 15$ \\
\hline
\end{tabular}

Tabelle 4.4: Mit Hilfe der Göttinger CRESU-Apparatur gemessene Geschwindigkeitskonstanten für die Reaktion des $\mathrm{OH}$ mit Isopren 


\section{Kapitel 5}

\section{Diskussion}

Wie im vorhergehenden Kapitel 4 beschrieben, werden für $\mathrm{C}_{2} \mathrm{H}_{2}$ und $\mathrm{C}_{2} \mathrm{H}_{4}$ nur die in dieser Arbeit gemessenen Geschwindigkeitskonstanten der Schwingungsrelaxation des Hydroxylradikals im schwingungsangeregten Zustand in Anwesenheit des Reaktionspartners diskutiert, die als obere Grenze für die Hochdruckgeschwindigkeitskonstante angesehen werden kann. Für die Reaktionen des Hydroxylradikals mit Propen und Isopren wurde angenommen, dass sie sich bei den untersuchten Bedingungen bereits im Hochdruckbereich befinden.

\subsection{Ethin $+\mathrm{OH}$}

In Tab. 5.1 sind die bisher gemessenen Hochdruckgeschwindigkeitskonstanten sowie deren Berechnung für die Reaktion Ethin $+\mathrm{OH}$ aufgeführt, in Abb. 5.1 finden sich diese Werte in einer doppelt logarithmischen Auftragung. Die beiden temperatur-

\begin{tabular}{|c|c|c|r|}
\hline $\begin{array}{c}\text { Temperatur } \\
{[\mathrm{K}]}\end{array}$ & $\begin{array}{c}k_{\infty} \\
{\left[10^{-12} \mathrm{~cm}^{3} \cdot \mathrm{s}^{-1}\right]}\end{array}$ & exp./theo. & Ref. \\
\hline \hline 296 & $0.969 \pm 0.030$ & exp. & {$[195]$} \\
$300-800$ & $38 \cdot \exp (-910 \mathrm{~K} / T)$ & exp. & {$[30]$} \\
$333-1273$ & $8.5 \cdot \exp (-1400 / T)$ & exp. & {$[66]$} \\
$250-2500$ & $2.5 \cdot 10^{-4} T^{1.7} \exp (-1000 / \mathrm{RT})$ & theo. & {$[72]$} \\
295 & $0.83 \pm 0.080$ & exp. & {$[60]$} \\
298 & 0.9 & exp. & {$[59]$} \\
$58-298$ & siehe Tab. 4.1 & exp. & diese Arbeit \\
$70-300$ & SACM & $\alpha / \beta=0.35$ & diese Arbeit \\
\hline
\end{tabular}

Tabelle 5.1: Geschwindigkeitskonstanten $k_{\infty}$ für die Reaktion des $\mathrm{OH}$ mit Ethin 


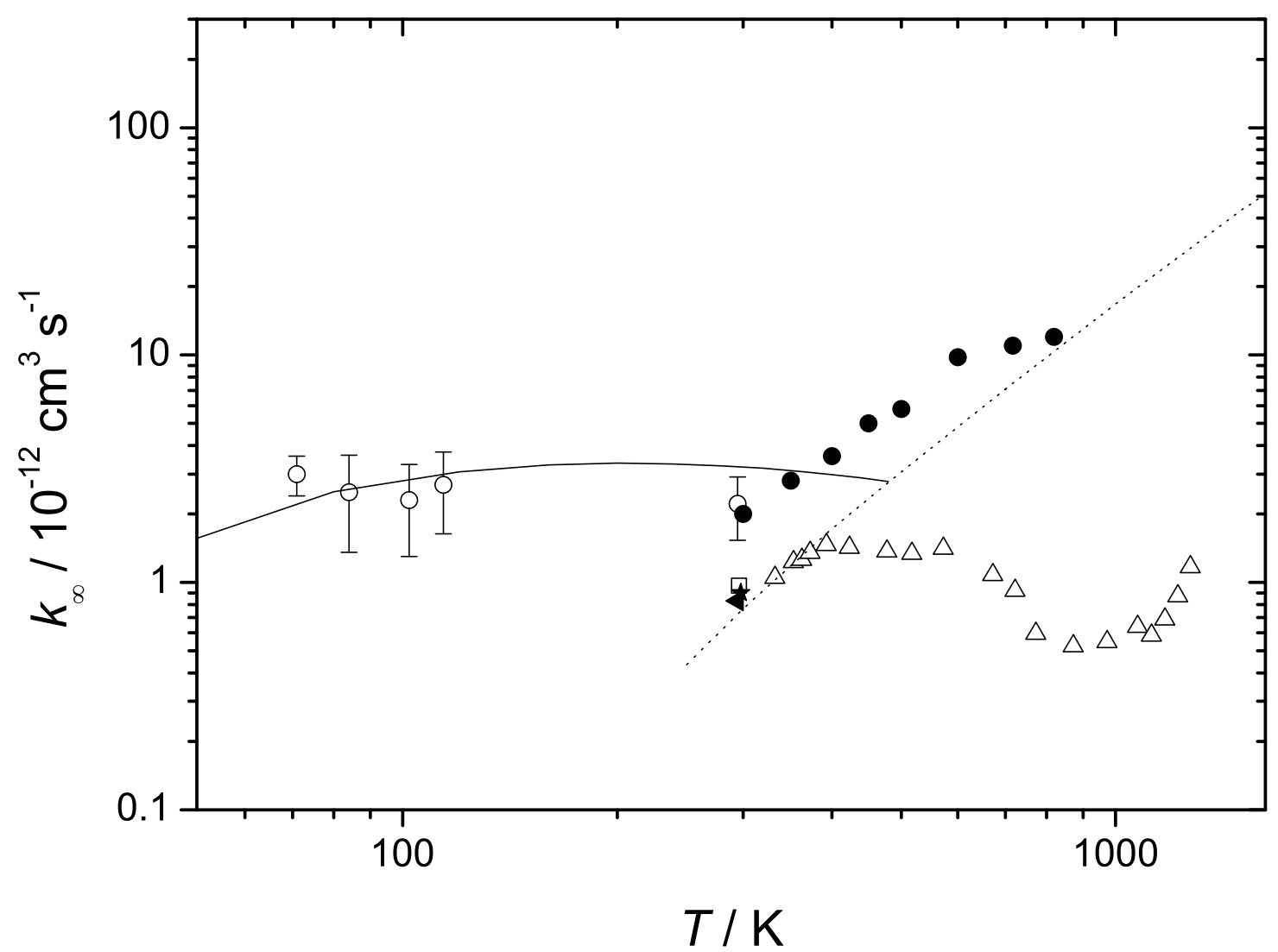

Abbildung 5.1: Geschwindigkeitskonstanten $k_{\infty}$ für die Reaktion des $\mathrm{OH}$ mit Ethin; Sørensen et al. [195], • Hippler et al. [30], $\triangle$ Liu et al. [66], ... Miller und Melius [72], * Schmidt et al. [60], « Wahner und Zetsch [59], o diese Arbeit, - SACM (diese Arbeit)

abhängigen Messungen von Hippler et al. $(\bullet)$ und Liu et al. $(\triangle)$ zeigen eine große Diskrepanz. Jedoch konkurriert ab ca. $700 \mathrm{~K}$ die Abstraktion eines Wasserstoffatoms mit der Addition des OH-Radikals an Ethin, daraus ergibt sich die S-förmige Kurve aus den Arbeiten von Liu et al. Außerdem wurden die Studien von Liu et al. bei Drücken von unter 1 bar durchgeführt, vermutlich ist der Hochdruckgrenzwert in dieser Arbeit noch nicht erreicht. Eine große Diskrepanz besteht ebenfalls zwischen den in der Literatur angegebenen Hochdruckgrenzwerten bei Raumtemperatur. Während in den Referenzen $[195,66,72,60,59]$ ein Wert von ca. $9 \cdot 10^{-12} \mathrm{~cm}^{3} \cdot \mathrm{s}^{-1}$ angegeben wird, haben Hippler et al. einen ungefähr doppelt so großen Wert bestimmt. Aufgrund dieses Unterschieds wird in Ref. [68] keine Empfehlung angegeben, eine plausible Erklärung findet sich in keiner der Veröffentlichungen. Die verschiedenen verwendeten Badgase sollten jedenfalls keine unterschiedlichen Werte 
liefern, da im Hochdruckgrenzfall die Energieübertragung durch Stöße keine Rolle mehr spielt. Möglicherweise ist die Extrapolation der bei niedrigeren Drücken (bis maximal 8 bar in allen anderen Arbeiten) durchgeführten Messungen der Geschwindigkeitskonstanten gegenüber den Messungen von Hippler et al. (bis maximal 80 bar) weniger exakt.

Der in dieser Arbeit bestimmte Raumtemperaturwert für $k_{\infty}$ stimmt sehr gut mit dem von Hippler et al. überein. Die bei Temperaturen unterhalb von $300 \mathrm{~K}$ gemessenen Werte zeigen sich weitestgehend temperaturunabhängig, ein Trend (positive oder negative Temperaturabhängigkeit) lässt sich innerhalb der experimentellen Fehler nicht erkennen. Aus der Anpassung der nach dem SAC-Modell berechneten Hochdruckgeschwindigkeitskonstanten (vgl. Abb. B.1) an die in dieser Arbeit gemessenen Werte ergibt sich ein Verhältnis von $\alpha / \beta=0.35$. Im Vergleich mit dem ,Standardwert" von $\alpha / \beta=0.5$ ist dies ein relativ niedriger Wert und deutet auf einen starren Übergangszustand und eine Barriere hin. Die Anpassung der SACM-Rechnung an die experimentellen Werte könnte auf einen leichten Abfall der Geschwindigkeitskonstanten zu tiefen Temperaturen hindeuten.

Dabei ist zu beachten, dass bei dem für die SACM-Berechnung in Abb. 5.1 ein $\alpha / \beta$-Wert von 0.35 verwendet wurde. In Abb. B.1 ist zu erkennen, dass zwischen einem Wert von 0.40 und 0.45 für $\alpha / \beta$ ein Wechsel in der Steigung des Graphen bei tiefen Temperaturen stattfindet, die Rechnungen reagieren also sehr empfindlich auf ein kleine Änderung des Interpolationsparameters $\alpha$. Die Anpassung der Parameter der SACM-Rechnung wurde jedoch so durchgeführt, dass eine gute Übereinstimmung der berechneten und experimentellen Werte der Geschwindigkeitskonstanten bei Raumtemperatur erreicht wurde. Für die korrekte Wiedergabe des temperaturunabhängigen Trends der Geschwindigkeitskonstanten werden etwas höhere Werte für $\alpha / \beta(0.40-0.45)$ benötigt. Damit steigen aber auch die Absolutwerte für die Geschwindigkeitskonstanten.

Der Vergleich der gemessenen Werte mit den Geschwindigkeitskonstanten, die die Messergebnisse am besten wiedergeben, deutet aber zumindest auf eine signifikante Barriere zur Bildung eines Adduktes aus Ethin und OH-Radikalen hin. Die Barriere zur Adduktbildung wurde mehrfach experimentell und theoretisch bestimmt. Hippler et al. [30] erhalten aus der Temperaturabhängigkeit der Geschwindigkeitskonstanten eine positive Aktivierungsenergie von $1.8 \mathrm{kcal} / \mathrm{mol}$, Liu et al. [66] erhalten einen Wert von $1.4 \mathrm{kcal} / \mathrm{mol}$. In zwei theoretischen Studien wurde die Aktivierungsenergie zu $1.4 \mathrm{kcal} / \mathrm{mol}$ [72] bzw. $1.6 \mathrm{kcal} / \mathrm{mol}$ [41] berechnet. Dabei wird der Übergangszustand in Ref. [41] durch ein noch fast lineares Ethin und ein mit dem Sauerstoff an eines der Kohlenstoffatome orientiertes Hydroxylradikal beschrieben, in Ref. [72] wird keine Aussage über die Art des Übergangszustandes gemacht. 


\begin{tabular}{|l|c|c|}
\hline Reaktion & $k_{\infty}\left[10^{-12} \mathrm{~cm}^{3} \cdot \mathrm{s}^{-1}\right]$ & Referenz \\
\hline \hline $\mathrm{OH}+$ Ethin & 0.97 & {$[195]$} \\
& 2.22 & diese Arbeit \\
$\mathrm{OH}+$ Propin & 5.71 & {$[61]$} \\
& 6.21 & {$[29]$} \\
$\mathrm{OH}+$ 1-Butin & 8.25 & {$[29]$} \\
$\mathrm{OH}+$ 2-Butin & 30.1 & {$[61]$} \\
\hline
\end{tabular}

Tabelle 5.2: Geschwindigkeitskonstanten $k_{\infty}$ für die Reaktion des $\mathrm{OH}$ mit einer Reihe von Alkinen $(T=298 \mathrm{~K})$

Vor dem Vergleich mit verwandten Reaktionen soll abschließend festgestellt werden, dass die Addition des Hydroxylradikals an Ethin die langsamste aller Reaktionen von OH-Radikalen mit Alkinen ist. Die wenigen Untersuchungen der entsprechenden Reaktion mit höheren homologen Alkinen bei Raumtemperatur liefern die in Tab. 5.2 angegebenen Geschwindigkeitskonstanten. Dabei ist der große Unterschied der Geschwindigkeitskonstanten der Addition des Hydroxylradikals an die beiden Butin-Isomere zu beachten. Möglicherweise spielt hier die unterschiedliche Stabilität der entstehenden Hydroxybutinylradikale eine Rolle.

\subsection{Ethen $+\mathrm{OH}$}

Um die in dieser Arbeit bestimmten Hochdruckgeschwindigkeitskonstanten für die Addition von Hydroxylradikalen an Ethen in einen Gesamtzusammenhang einordnen zu können, sind die bisher experimentell und theoretisch bestimmten Werte in Tab. 5.3 zusammengefasst und in Abb. 5.2 aufgetragen. Ähnlich den bisherigen experimentellen Daten für den Hochdruckbereich der Reaktion von Ethin mit $\mathrm{OH}-$ Radikalen tritt für die Addition des Hydroxylradikals an Ethen eine Diskrepanz zwischen den Werten von Hippler et al. [30] und den restlichen Werten auf [39,66,28,27]. Eine plausible Erklärung sind die angewendeten Drücke von bis zu 1 bar für die letztzitierten Messungen, die Untersuchungen von Hippler et al. wurden bei 40 bzw. 140 bar durchgeführt und sollten deshalb die verlässlichere Extrapolation zu Hochdruckgeschwindigkeitskonstanten darstellen. Die Hochdruckgrenzwerte bei Raumtemperatur sowohl aus experimentellen als auch der theoretischen Untersuchung [47] stimmen mit dem in dieser Arbeit aus der Relaxationsgeschwindigkeitskonstanten abgeschätzten $k_{\infty}$ gut überein. 


\begin{tabular}{|c|c|c|r|}
\hline $\begin{array}{c}\text { Temperatur } \\
{[\mathrm{K}]}\end{array}$ & $\begin{array}{c}k_{\infty} \\
{\left[10^{-12} \mathrm{~cm}^{3} \cdot \mathrm{s}^{-1}\right]}\end{array}$ & exp./theo. & Ref. \\
\hline \hline 298 & 11.7 & theo. & {$[47]$} \\
$300-600$ & - & exp.(40 bar $)$ & {$[30]$} \\
$660-800$ & - & exp.(140 bar $)$ & {$[30]$} \\
$544-673$ & $0.4 \cdot \exp (1200 / T)$ & exp. & {$[39]$} \\
$343-1173$ & $(1.65 \exp (480 / T)$ & exp. & {$[66]$} \\
295 & $(8.5 \pm 0.6)$ & exp. & {$[28]$} \\
$296-524$ & $2.0 \cdot \exp [320 / T]$ & exp. & {$[27]$} \\
$62-298$ & siehe Tab. 4.2 & exp. & diese Arbeit \\
$30-300$ & SACM & $\alpha / \beta=0.58$ & diese Arbeit \\
\hline
\end{tabular}

Tabelle 5.3: Geschwindigkeitskonstanten $k_{\infty}$ für die Reaktion des $\mathrm{OH}$ mit Ethen

Die unterhalb von $300 \mathrm{~K}$ bestimmten Geschwindigkeitskonstanten zeigen eine deutlich negative Temperaturabhängigkeit und führen somit den Trend, den alle Messungen oberhalb von $300 \mathrm{~K}$ zeigen, fort. Die beste Übereinstimmung der SACMRechnung mit den bisher und den in dieser Arbeit bestimmten Daten konnte mit $\alpha / \beta=0.58$ erzielt werden. Um eine noch bessere Übereinstimmung mit den experimentellen Daten unterhalb von $300 \mathrm{~K}$ zu erreichen, müsste das Verhältnis von $\alpha / \beta$ leicht abgesenkt werden (z. B. auf 0.55). Resultat dieser Absenkung wäre aber eine schlechtere Reproduktion der Geschwindigkeitskonstanten bei $300 \mathrm{~K}$. Deutlich wird auch in diesem Fall die Empfindlichkeit der Temperaturabhängigkeit und der Absolutwerte der Hochdruckgeschwindigkeitskonstanten von $\alpha / \beta$.

Das für diese Reaktion angepasste Verhältnis von $\alpha / \beta$ von 0.58 liegt über dem „Standardwert" von 0.5 und deutet damit auf einen eher lockeren und vom Addukt aus gesehen späten Übergangszustand hin. Diese Einschätzung wird durch die Berechnungen von Villà et al. [45] gestützt, da sich in dieser Arbeit die meisten Frequenzen auf dem Weg von den getrennten Edukten zum Übergangszustand nicht signifikant ändern.

Die experimentellen temperaturabhängigen Untersuchungen liefern Aktivierungsenergien von $-2.3 \mathrm{kcal} / \mathrm{mol} \quad[39], \quad-1.74 \mathrm{kcal} / \mathrm{mol} \quad[66], \quad-0.6 \mathrm{kcal} / \mathrm{mol}$ [27], $-0.77 \mathrm{kcal} / \mathrm{mol}$ [21] und $-0.9 \mathrm{kcal} / \mathrm{mol}$ [12]. Da die extrapolierten Hochdruckgeschwindigkeitskonstanten von Hippler et al. keine Temperaturabhängigkeit zeigten, interpretierten sie die Reaktion als barrierelos. Die etwas größeren negativen Werte von -2.3 bzw. $-1.74 \mathrm{kcal} / \mathrm{mol}[39,66]$ lassen sich durch die ungenaue Extrapolation der Hochdruckgeschwindigkeitskonstanten erklären. Als Mittelwert der scheinbaren experimentellen Aktivierungsenergien ergibt sich ein Wert von ca. $-1 \mathrm{kcal} / \mathrm{mol}$. Die 


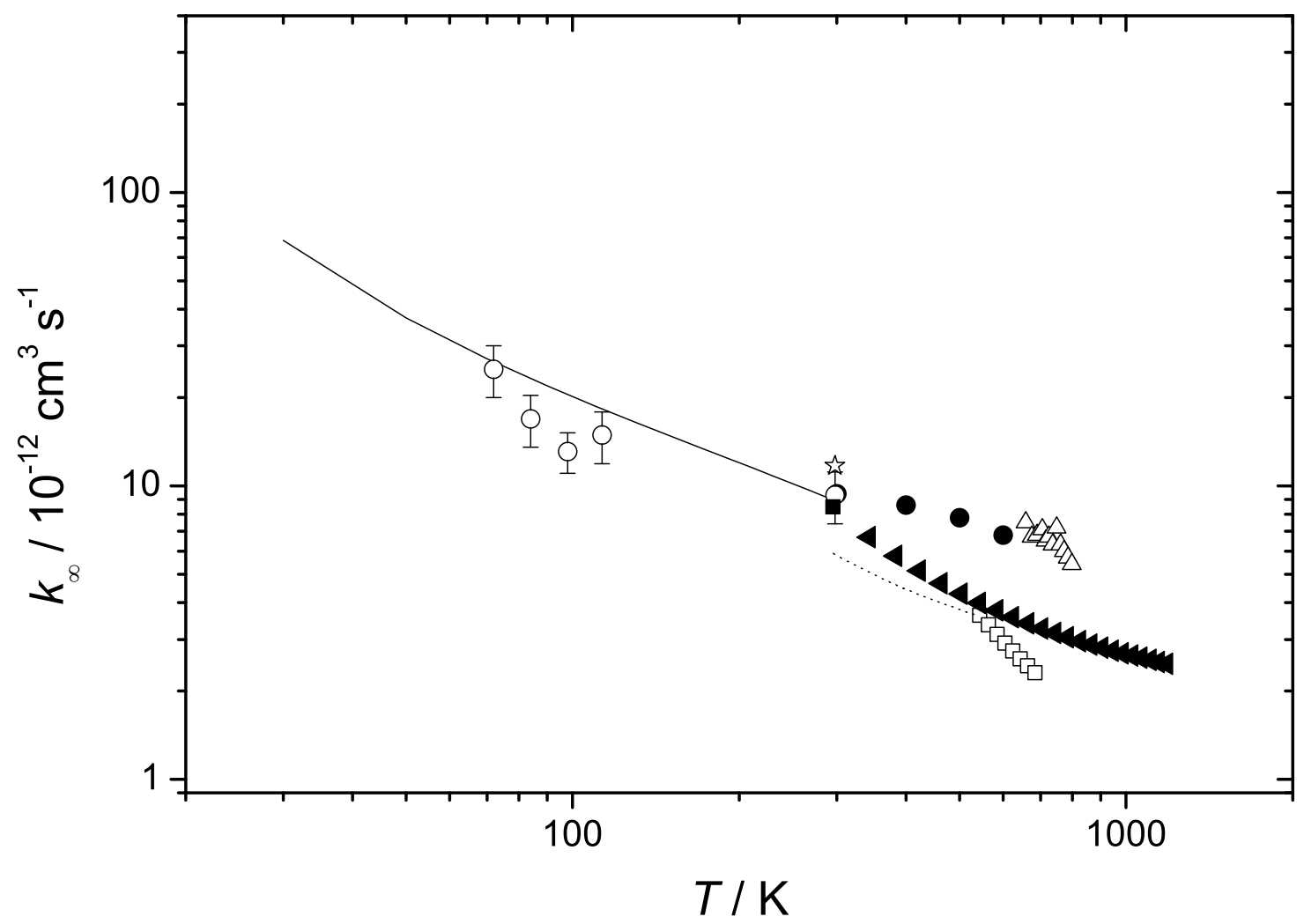

Abbildung 5.2: Doppelt logarithmische Auftragung der bisherigen Geschwindigkeitskonstanten für die Reaktion des $\mathrm{OH}$ mit Ethen; $\star$ AlvarezIdaboy et al. [47], • bzw. $\triangle$ Hippler et al. (40 bzw. 140 bar) [30], Diaz-Costa et al. [39], \ Liu et al. [66], ם Klein [28], ‥ Zellner et al. [27], o diese Arbeit, - SACM (diese Arbeit)

Berechnungen zur Addition des Hydroxylradikals an Ethen sind sehr zahlreich. Dabei wurde der Energieunterschied der Barriere relativ zu den Edukten berechnet. Es wurden Werte von $-2.1 \mathrm{kcal} / \mathrm{mol}$ [50], $-1.79 \mathrm{kcal} / \mathrm{mol}$ [49], $-0.54 \mathrm{kcal} / \mathrm{mol}$ [47], $-0.92 \mathrm{kcal} / \mathrm{mol}$ [45] und $-0.9 \mathrm{kcal} / \mathrm{mol}$ [41] berechnet, laut Ref. [48] verläuft die Addition von OH-Radikalen an Ethen völlig ohne Barriere, das Hydroxyethenylradikal wird direkt gebildet und durch Stöße stabilisiert.

In der Serie der Reaktionen von Hydroxylradikalen mit substituierten Alkenen verläuft die entsprechende Reaktion von Ethen am langsamsten. Gemeinsam haben alle Reaktionen methylsubstituierter Alkene negative Aktivierungsenergien [47]. Die Einführung einer Methylgruppe an eines der Kohlenstoffatome der Doppelbindung erhöht die Geschwindigkeitskonstante merklich, sie verdreifacht sich etwa 


\begin{tabular}{|l|c|}
\hline Reaktion & $k\left[10^{-12} \mathrm{~cm}^{3} \cdot \mathrm{s}^{-1}\right]$ \\
\hline \hline $\mathrm{OH}+$ Ethen & 8.52 \\
$\mathrm{OH}+$ Propen & 26.3 \\
$\mathrm{OH}+$ Methylpropen & 51.4 \\
$\mathrm{OH}+$ cis-2-Buten & 56.4 \\
$\mathrm{OH}+$ 2-Methyl-2-Buten & 86.9 \\
$\mathrm{OH}+$ 2-Methyl-1,3-Butadien & 99.7 \\
$\mathrm{OH}+2,3-$ dimethyl-2-Buten & 110 \\
\hline
\end{tabular}

Tabelle 5.4: Geschwindigkeitskonstanten für die Reaktion des $\mathrm{OH}$ mit einer Reihe von Alkenen $(T=298 \mathrm{~K})$; alle Werte wurden Ref. [196] entnommen

von Ethen nach Propen und verdoppelt sich von Propen nach Methylpropen. Einen kleineren Effekt bewirkt die Einführung einer Ethyl- anstatt einer Methylgruppe; ebenfalls spielt es für die Geschwindigkeit der Reaktion keine entscheidende Rolle, an welcher Stelle die Methylgruppe eingeführt wird. Die Substituenten haben somit wahrscheinlich einen Einfluss auf die Stabilität der Übergangszustände und $\beta$-Hydroxyalkylradikale, der sich möglicherweise auf elektronische Ursachen zurückführen lässt. In Ref. [47] wurde gefunden, dass durch eine Methylgruppe - mit ihren „positiv geladenen“ Wasserstoffatomen - die Addition des Hydroxylradikals an das höher substituierte Kohlenstoffatom favorisiert wird. Anhand der Rechnungen von Diaz-Costa et al. [6] für die Hydroxypropenylradikale lässt sich diese Vermutung bestätigen, das Addukt mit der zentralen Position der Hydroxylgruppe ist etwas stabiler als das entsprechende mit der terminalen Position. Die Geschwindigkeitskonstanten für die Reaktionen des Hydroxylradikals mit verschiedenen Alkenen bei $298 \mathrm{~K}$ sind in Tab. 5.4 aufgeführt.

\subsection{Propen $+\mathrm{OH}$}

Die bisher bestimmten experimentellen Daten für die Reaktion des Propen mit OHRadikalen sind in Tab. 5.5, die mit Hilfe der Göttinger CRESU-Apparatur gemessenen Werte in Tab. 4.3 aufgeführt. Alle Daten zusammen mit den Ergebnissen der durchgeführten SACM-Rechnungen sind in Abb. 5.4 dargestellt. Aus den zahlreichen Werten der Geschwindigkeitskonstante bei Raumtemperatur in der Literatur wurden hier nur zwei Arbeiten [28,29] ausgewählt, da sämtliche Werte innerhalb der Fehler übereinstimmen. Die temperaturabhängigen Daten aus Ref. [79] wurden hier aufgrund der Übersichtlichkeit nicht eingezeichnet. 


\begin{tabular}{|c|c|c|}
\hline $\begin{array}{c}\text { Temperatur } \\
{[\mathrm{K}]}\end{array}$ & $k$ & Ref. \\
\hline \hline 103 & $\left.81 \pm 10^{-12} \mathrm{~cm}^{3} \cdot \mathrm{s}^{-1}\right]$ & \\
295 & $30 \pm 2$ & {$[78]$} \\
295 & 26.3 & {$[28]$} \\
\hline
\end{tabular}

Tabelle 5.5: Bisherige Geschwindigkeitskonstanten für die Reaktion des $\mathrm{OH}$ mit Propen

Die in dieser Arbeit bestimmten Geschwindigkeitskonstanten bei Raumtemperatur befinden sich in exzellenter Übereinstimmung mit den bisherigen Arbeiten. Zunächst steigt die Geschwindigkeitskonstante der betrachteten Reaktion unterhalb von Raumtemperatur bis ca. $120 \mathrm{~K}$ an, unterhalb von $100 \mathrm{~K}$ ist sie nahezu konstant, möglicherweise fällt sie aber auch leicht ab. Dies lässt sich innerhalb der experimentellen Fehler nicht entscheiden. Ebenfalls in guter Übereinstimmung zeigen sich die von Leone et al. [78] bei $103 \mathrm{~K}$ bestimmte Geschwindigkeitskonstante mit in dieser Arbeit ermittelten Wert. Die Ergebnisse zur Modellierung der Reaktion mit Hilfe des SAC-Modells sind in Abb. 5.4 ebenfalls dargestellt. Zunächst wurde der Zerfall für die beiden isomeren Addukte getrennt berechnet (Striche: Addukt 1, Punkte: Addukt 2, vgl. Abb. 1.3). Aufgrund der gerinfügig unterschiedlichen Stabilität der beiden Isomere (Energiedifferenz ca. $2 \mathrm{kcal} / \mathrm{mol}$ ) und der individuellen Anpassung des Verhältnisses von $\alpha / \beta$ ergeben sich leicht unterschiedliche Temperaturabhängigkeiten.

Die SACM-Berechnung des Adduktes 1 (zentrale Position der Hydroxylgruppe) wurde mit einem Wert von 0.56 für $\alpha / \beta$ angepasst, für das Addukt 2 (terminaler Angriff des Hydroxylradikals) wurde ein Wert von 0.62 für $\alpha / \beta$ ermittelt. Die beiden berechneten Geschwindigkeitskonstanten wurden für jede Temperatur gemittelt, der Mittelwert ist als durchgezogene Linie dargestellt. Alternativ können die berechneten individuellen Geschwindigkeitskonstanten der einzelnen Isomere addiert werden,

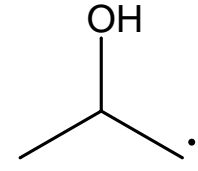

1

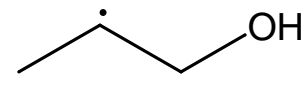

2

Abbildung 5.3: Bei der Reaktion des OH-Radikals mit Propen entstehende Isomere 


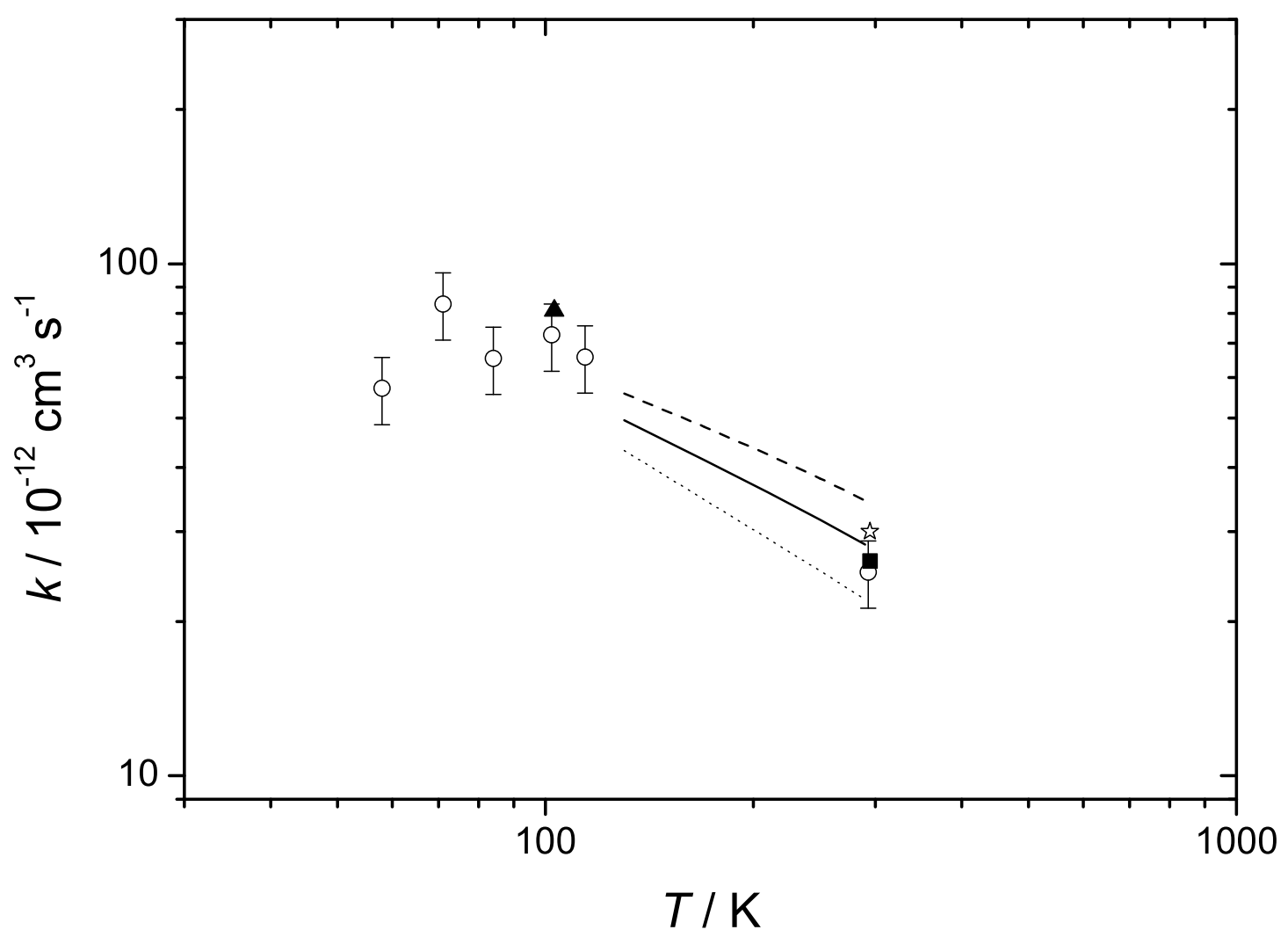

Abbildung 5.4: Doppelt logarithmische Auftragung der bisherigen Geschwindigkeitskonstanten für die Reaktion des $\mathrm{OH}$ mit Propen; $\boldsymbol{\Delta}$ Leone et al. [78], * Klein et al. [28], - Atkinson und Aschmann [29], o diese Arbeit, - SACM (diese Arbeit)

allerdings wäre eine Absenkung des $\alpha / \beta$-Wertes auf ca. 0.5 nötig, um die experimentellen Daten zufriedenstellend wiederzugeben.

Während die Abschätzung nach dem SAC-Modell bis zu extrem tiefen Temperaturen weiterhin eine stark negative Temperaturabhänigkeit zeigt, bleiben die experimentelle Werte innerhalb der Fehlergrenzen ab ca. $100 \mathrm{~K}$ konstant. Die Werte des Verhältnisses von $\alpha / \beta$, das ein Maß für die Eigenschaften des Übergangszustandes ist, deuten für beide Isomere auf einen eher lockeren Übergangszustand hin. In der Literatur wird stets eine Barriere postuliert, die sich zwischen $-1.00 \mathrm{kcal} / \mathrm{mol}$ [196] und $-2.06 \mathrm{kcal} / \mathrm{mol}$ [47] unterhalb der Energie der Edukte befinden soll. Ähnliche Temperaturabhängigkeiten zeigen die verwandten Reaktionen des Hydroxylradikals mit 1-Buten sowie E- und Z-2-Buten im Temperaturbereich von 23-295 K. Sie zeigen ebenfalls eine schwächere negative Temperaturabhängigkeit zu tieferen Temperatu- 
ren, eine Stagnation oder sogar eine Umkehr der Abhängigkeit ist jedoch nicht zu beobachten.

\subsection{Isopren $+\mathrm{OH}$}

In den Tabellen 5.6 und 4.4 sind die bisher bestimmten bzw. in dieser Arbeit gemessenen Geschwindigkeitkonstanten für die Reaktion des Hydroxylradikals mit 2-Methyl-1,3-Butadien (Isopren) aufgeführt. Auf weitere Daten zur untersuchten Reaktion bei Raumtemperatur, die eine Streuung innerhalb der angegebenen Werte $[31,8,85]$ bei Raumtemperatur darstellen, wurde der Übersichtlichkeit halber verzichtet. Die negative Temperaturabhängigkeit der Geschwindigkeitskonstanten wurde sowohl in den Arbeiten von Pitts et al. [86] als auch von Gill et al. [87] in guter Übereinstimmung der Werte beobachtet.

Die im Rahmen dieser Arbeit bestimmte Geschwindigkeitskonstante bei Raumtemperatur befindet sich in sehr guter Übereinstimmung mit den bisherigen Messungen. Unterhalb von Raumtemperatur wird die Reaktion zunächst mit sinkender Temperatur schneller und setzt damit den Trend der Temperaturabhängigkeit oberhalb von $300 \mathrm{~K}$ fort. Ab ca. $100 \mathrm{~K}$ wird sie jedoch deutlich langsamer, zwischen 120 und $60 \mathrm{~K}$ geht die Geschwindigkeitskonstante sogar wieder auf ein Drittel des Maximalwertes bei ca. $100 \mathrm{~K}$ zurück. Die durchgeführten Messungen zeigen also ein deutliches Maximum knapp oberhalb von $100 \mathrm{~K}$.

Zur Diskussion der durchgeführten SACM-Rechnungen werden die einzelnen Isomere des OH-Isoprenadduktes (vgl. Abb. 5.6) zunächst getrennt voneinander betrachtet. Die Isomere 1 und 3 werden durch eine Delokalisierung des ungepaarten Elektrons über drei Kohlenstoffatome stabilisiert. Deswegen liegen sie energetisch relativ zu den Edukten ca. $13 \mathrm{kcal} / \mathrm{mol}$ niedriger als die Isomere 2 und 4. In Ref. [89] wurden

\begin{tabular}{|c|c|r|}
\hline $\begin{array}{c}\text { Temperatur } \\
{[\mathrm{K}]}\end{array}$ & $k$ & Ref. \\
\hline \hline $299-422$ & $23.6 \cdot \exp (409 \pm 28)$ & {$[86]$} \\
300 & $110 \pm 4$ & {$[31]$} \\
295 & $99 \pm 5$ & {$[8]$} \\
298 & $85.6 \pm 2.6$ & {$[85]$} \\
$298-363$ & $25.6 \cdot \exp (408 \pm 42)$ & {$[87]$} \\
\hline
\end{tabular}

Tabelle 5.6: Bisherige Geschwindigkeitskonstanten für die Reaktion des OH mit Isopren 


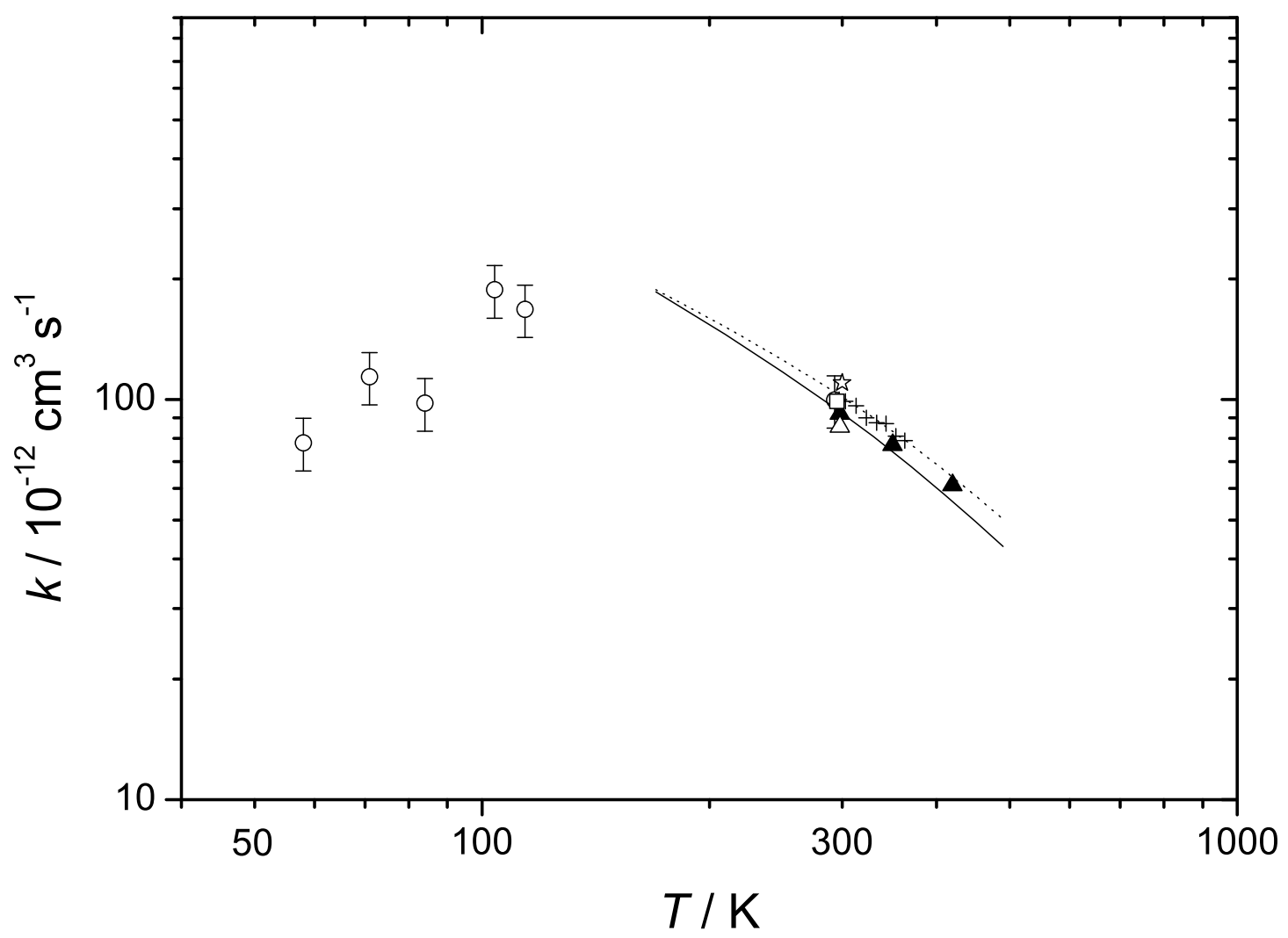

Abbildung 5.5: Doppelt logarithmische Auftragung der bisherigen Geschwindigkeitskonstanten für die Reaktion des $\mathrm{OH}$ mit Isopren; $\boldsymbol{\Delta}$ Kleindienst et al. [86], ^ Chuong und Stevens [31], $\square$ McGivern et al. [8], $\triangle$ Hynes et al. [85], + Gill und Hites [87], o diese Arbeit, - bzw. ... SACM (diese Arbeit)

die Geschwindigkeitskonstanten der Bildung der verschiedenen Isomeren berechnet, sie erhielten ein Isomerenverhältnis von $0.56: 0.023: 0.046: 0.37$ (Isomer $1: 2: 3: 4$ ). Somit tragen die Isomere 2 und 4, in denen sich die Hydroxylgruppe sich in 2- bzw. 3Position befindet und das ungepaarte Elektron sich in terminaler Position befindet, aufgrund ihrer geringeren Stabilität kaum zur Reaktion bei. Deshalb wurden lediglich die SACM-Rechnungen für die Isomere 1 und 3 an die experimentellen Daten angepasst, die Ergebnisse sind in Abb. 5.5 zu sehen. Dabei sind die Geschwindigkeitskonstanten für Isomer 1 als Punkte, die von Isomer 2 als durchgezogenen Linie dargestellt. Auf eine Mittelung der Werte wurde hier verzichtet. Auch in diesem Fall bietet sich eine Aufsummierung der Geschwindigkeitskonstanten der einzelnen Isomere an, wie im Fall des Propens müsste $\alpha / \beta$ für eine gute Reproduktion der 


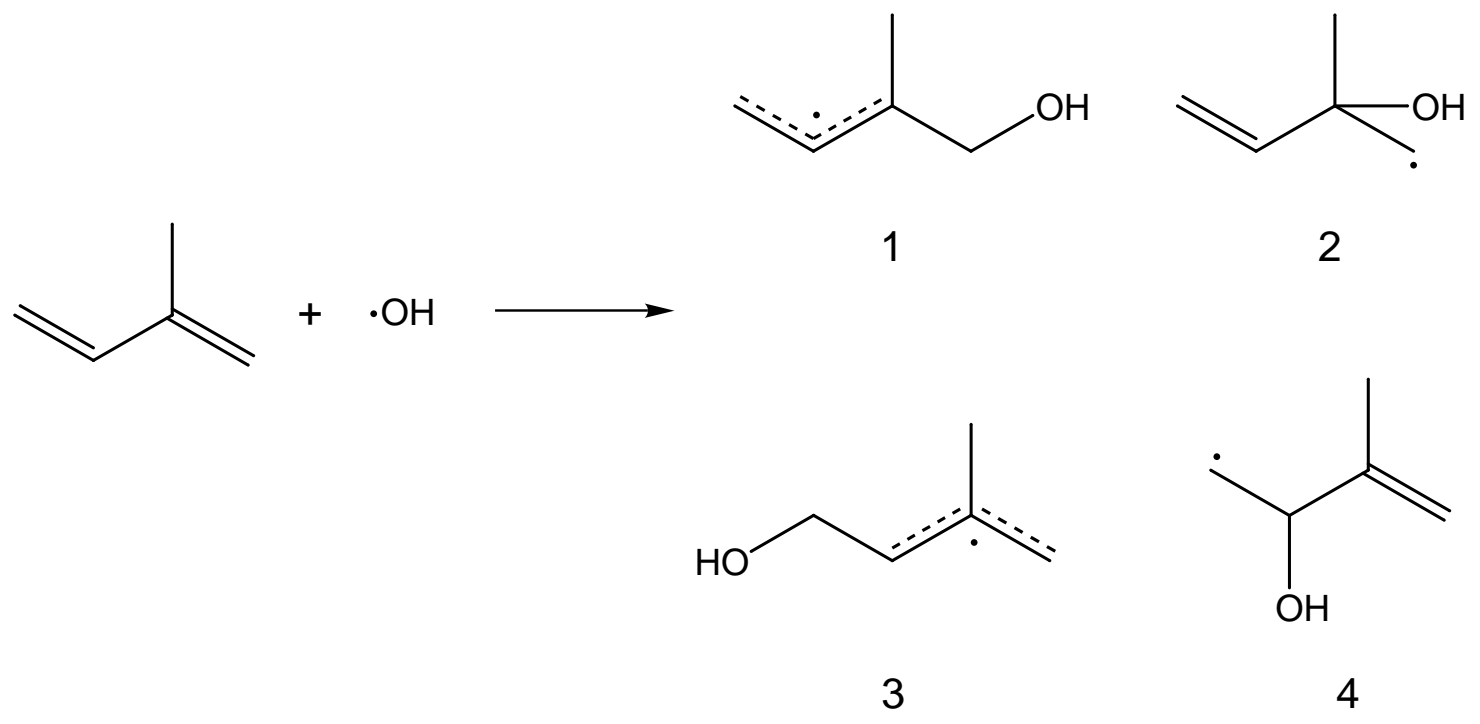

Abbildung 5.6: Bei der Reaktion des OH-Radikals mit Isopren entstehende Isomere

experimentellen Werte auf ca. 0.5 abgesenkt werden. Die beste Reproduktion der bisherigen Daten oberhalb von $300 \mathrm{~K}$ durch die SACM-Rechnungen gelang mit einem $\alpha / \beta$-Verhältnis von 0.82 für Isomer 1und 2. Diese großen Werte von $\alpha / \beta$ deuten auf einen lockeren Übergangszustand hin. Bemerkenswert ist die gute Wiedergabe der experimentell bestimmten Temperaturabhängigkeit oberhalb von $300 \mathrm{~K}$. Dies deutet auf eine gute Beschreibung der Reaktion durch das SACM-Modell hin.

\subsection{Vergleich der untersuchten Reaktionen}

Ein Vergleich der mit Hilfe der Göttinger CRESU-Apparatur bestimmten Geschwindigkeitskonstanten für die (OH + Ethin)-, $(\mathrm{OH}+$ Ethen $)-$, $(\mathrm{OH}+$ Propen $)$ - und $(\mathrm{OH}+$ Isopren)-Reaktion sowie der bereits untersuchten Reaktionen verschiedener Buten-Isomer-Reaktionen [146] findet sich in Abb. 5.7. Da sowohl die Absolutwerte als auch die Temperaturabhängigkeit der verschiedenen Buten-Isomere (1-Buten, cis-2-Buten, trans-2-Buten) (vgl. Ref. [146]) sehr ähnlich sind, werden in Abb. 5.7 nur die Werte für die Reaktion eines Isomers aufgeführt. Die durchgezogenen Linien in Abb. 5.7 sind Anpassungen der experimentellen Daten an Gl. 5.1.

$$
k(T)=A(T / \mathrm{K})^{n} \exp (\theta / T) .
$$

Dabei sind $A, n$ und $\theta$ anpassbare Parameter, die Werte für die verschiedenen Reaktionen sind in Tabelle 5.7 angegeben. Gl. 5.1 wird häufig für die Beschreibung 


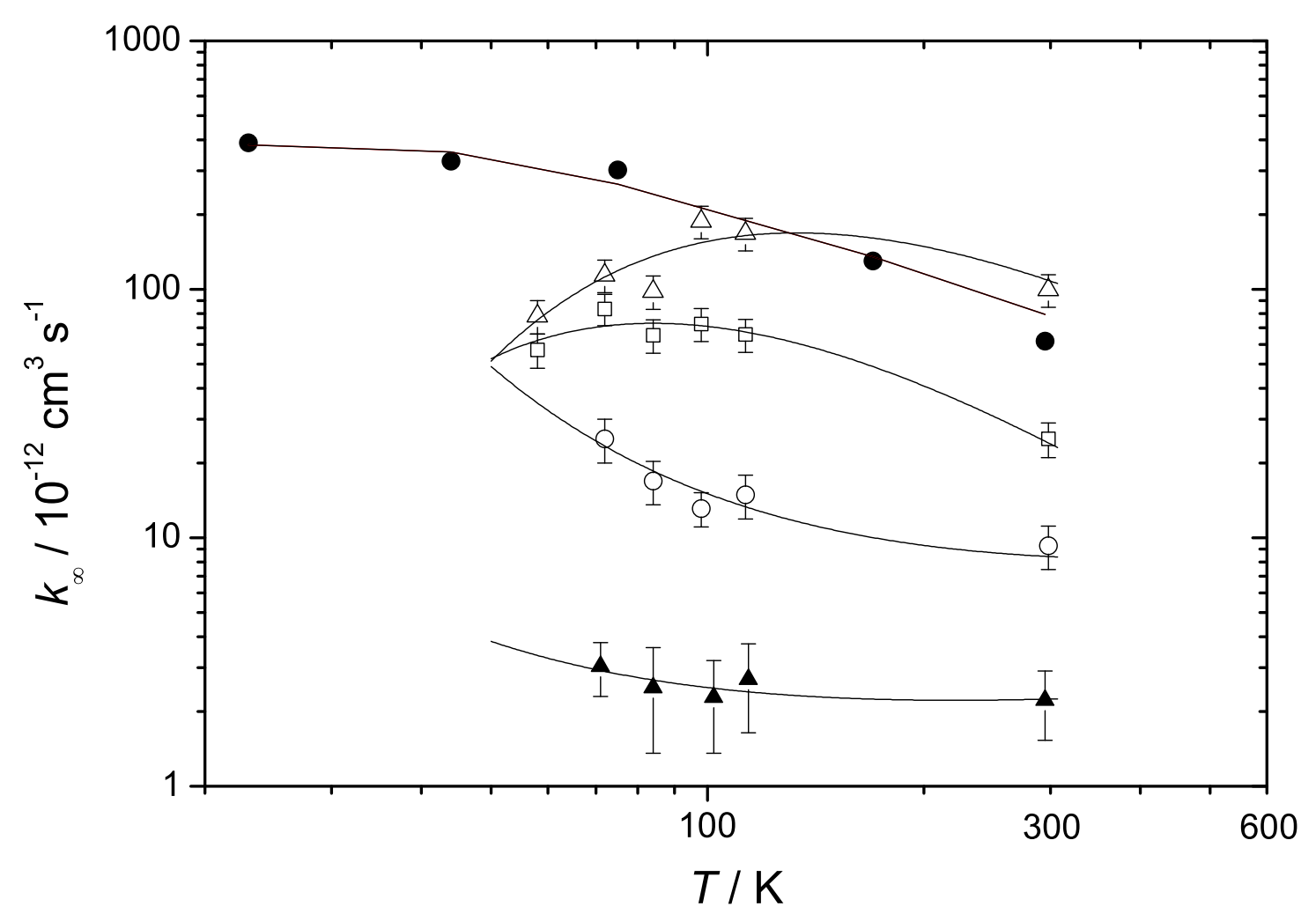

Abbildung 5.7: Die Reaktionen des Hydroxylradikals mit verschiedenen Alkenen bei tiefen Temperaturen; $\boldsymbol{\Delta}: \mathrm{OH}+$ Ethin, $\circ: \mathrm{OH}+$ Ethen, $\square: \mathrm{OH}+$ Propen, $\triangle: \mathrm{OH}+$ Isopren, $\bullet: \mathrm{OH}+$ cis-2-Buten [146]

\begin{tabular}{|l|ccc|}
\hline Reaktion & $A\left[\mathrm{~cm}^{3} \cdot \mathrm{s}^{-1}\right]$ & $n$ & $\Theta[\mathrm{K}]$ \\
\hline \hline OH + cis-2-Buten & $4.88 \cdot 10^{-8}$ & -1.11 & -31.4 \\
OH + Isopren & $4.81 \cdot 10^{-6}$ & -1.74 & -232 \\
OH + Propen & $4.38 \cdot 10^{-6}$ & -2.03 & -170 \\
OH + Ethen & $8.50 \cdot 10^{-13}$ & 0.32 & 140 \\
OH + Ethin & $3.38 \cdot 10^{-13}$ & 0.29 & 63.8 \\
\hline
\end{tabular}

Tabelle 5.7: Parameter für die Anpassung der Geschwindigkeitskonstanten für die Reaktion des $\mathrm{OH}$ mit ungesättigten Kohlenwasserstoffen an Gl. 5.1 
der Temperaturabhängigkeit der Geschwindigkeitskonstanten bei tiefen Temperaturen benutzt und dient als Grundlage für astrochemische Modelle [152]. Die Anpassung ist jedoch rein empirisch, eine theoretische Bedeutung der Parameter $n$ und $\theta$ ist nicht vorhanden. Deshalb sollte die oben angegebene Formel auch nicht oberhalb von $300 \mathrm{~K}$ verwendet werden. Die in Abb. 5.7 aufgetragenen Geschwindigkeitskonstanten zeigen die Abhängigkeit der Geschwindigkeitskonstanten der Reaktion des Hydroxylradikals mit ungesättigten Kohlenwasserstoffen von dem Substitutionsgrad des Alkens bzw. Alkins. Die Geschwindigkeitskonstanten variieren dabei über zwei Größenordnungen. Auch die Temperaturabhängigkeit zeigt große Unterschiede. Während die Ausbildung eines Maximums für die Reaktionen des Isoprens und wahrscheinlich auch des Propens mit $\mathrm{OH}$ beobachtet wurde, ist für die entsprechende Reaktion des Ethens bis zu den gemessenen Temperaturen ein monotoner Anstieg zu sehen. Die Reaktion des Hydroxylradikals mit Ethin zeigt im untersuchten Temperaturbereich so gut wie keine Temperaturabhängigkeit. Die gleiche Systematik wie in der Temperaturabhängigkeit lässt sich auch in den Geschwindigkeitskonstanten bei Raumtemperatur erkennen, die in der Reihen von Ethin, Ethen, Propen und Isopren deutlich zunehmen.

Allen betrachteten Reaktionen gemeinsam ist die mehr oder weniger ausgeprägte negative Temperaturabhängigkeit der Geschwindigkeitskonstanten unterhalb von ca. 300 K. Eigenschaften der Potentialfläche, die zu einem solchen Verhalten führen können, werden im nächsten Absatz vorgestellt. Anschließend wird erörtert, welche Effekte für die beobachtete Temperaturabhängigkeit der einzelnen Reaktionen verantwortlich sein könnten.

1. Als Erklärungsansatz für die negative Temperaturabhängigkeit der Geschwindigkeitskonstanten einer Reaktion wird häufig der Einfluss des langreichweitigen Teils der Potentialfläche herangezogen. Der Einfang der Reaktanden bei Reaktionen von Radikalen mit ungesättigten Kohlenwasserstoffen findet häufig schon bei großen Abständen der Reaktanden statt. Bei dieser Annäherung bei großen Abständen wirken vor allem elektrostatische Kräfte, d. h. die Geschwindigkeit der Reaktion wird allein durch die Dipol- bzw. eventuell vorhandenen Quadrupolmomente der Reaktanden bestimmt. Aufgrund der geringeren thermischen Energien bei tiefen Temperaturen können diese Kräfte effektiver wirken, der Einfang der Reaktanden wird erleichtert.

2. Nach dem Einfang der Reaktanden wird in vielen Arbeiten die Bildung eines $\pi$-Komplexes postuliert, bevor es zu einer kovalenten Bindung in einem Addukt kommt. Dieser Komplex kommt durch eine Wechselwirkung der $\pi$-Orbitale des OH-Radikals und der CC-Doppel- bzw. Dreifachbindung des ungesättigten Kohlenwasserstoffs zustande. Der auch als lockerer Cluster bezeichnete 
$\pi$-Komplex zwischen $\mathrm{OH}$ und Ethin wurde in Ref. [41] berechnet. Das elektrophile Wasserstoffatom ist im Komplex senkrecht zur Doppelbindung des Kohlenwasserstoffs ausgerichtet (Abb. 5.8). Im Fall des Ethins wurde die Stabilität des $\pi$-Komplexes zu $2.93 \mathrm{kcal} / \mathrm{mol}$ bestimmt [41]. Wegen der postulierten Positionierung vor dem eigentlichen Übergangszustand auf der Reaktionskoordinate (vgl. Abb. 5.9) wird er auch als präreaktiver Komplex bezeichnet.
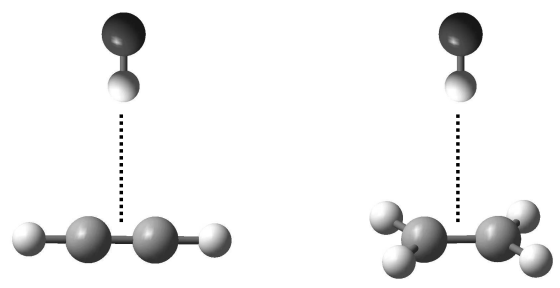

Abbildung 5.8: $\pi$-Komplex für die Addition von $\mathrm{OH}$ an Ethin bzw. Ethen

Verwandte Komplexe dieser Art wurden von Hansen et al. untersucht [197]. In diesen Arbeiten werden Cluster von $\mathrm{HO}_{2}$ - und $\mathrm{OH}$-Radikalen mit verschiedenen organischen Molekülen berechnet, die teilweise eine bemerkenswert hohe Stabilität aufweisen. Es wurden Bindungsenergien von bis zu $13 \mathrm{kcal} / \mathrm{mol}$ (z. B. für den $\mathrm{HO}_{2} \cdot-\mathrm{CH}_{3} \mathrm{NH}_{2}$-Komplex) berechnet. Die in dieser Arbeit in Frage kommenden $\pi$-Komplexe wurden jedoch nicht behandelt. In einer verwandten Reaktion des Triplett-Sauerstoffs $\left(\mathrm{O}\left({ }^{3} \mathrm{P}\right)\right)$ mit ungesättigten Kohlenwasserstoffen wird ebenfalls die Bildung eines präreaktiven Komplexes postuliert [198], der einige wenige $\mathrm{kcal} / \mathrm{mol}$ stabil sein soll. Im präreaktiven $\pi$ Komplex aus dem OH-Radikal und Ethen orientiert sich das Hydroxylradikal ebenfalls mit dem Wasserstoffatom senkrecht zur Doppelbindung (vgl. Abb. 5.8), die beiden am Komplex beteiligten Moleküle sind in ihrer Geometrie und damit auch in ihren Frequenzen weitestgehend ungestört [49]. Dabei hat das Wasserstoffatom des Hydroxylradikals laut Ref. [49] einen Abstand von ca. 2.6 Åzur Doppelbindung, das Radikalzentrum ist am Sauerstoffatom lokalisiert.

In Ref. [6] wurde mit ab initio-Methoden ein Energieminimum im Potential der Reaktion des Hydroxylradikals mit Propen gefunden, welches ebenfalls einem $\pi$-Komplex zugeordnet wurde. Bezogen auf die Temperaturabhängigkeit der Geschwindigkeitskonstanten können nun folgende Überlegungen angestellt werden. Sollte der $\pi$-Komplex nach dem Einfang der Reaktanden gebildet werden, so werden bei geringerer Temperatur sicherlich weniger dieser zunächst gebildeten Komplexe zu den Edukten zurückdissoziieren. Da der Komplex also bei geringerer Temperatur effektiver stabilisiert werden kann, nimmt die Geschwindigkeitskonstante für die Komplexbildung zu. 
Um zum Addukt (auch als $\sigma$-Komplex bezeichnet) zu gelangen (vgl. Abb. 5.9), muss sich das Hydroxylradikal umorientieren. Von der Orientierung des Wasserstoffatoms senkrecht zur Doppel- bzw. Dreifachbindung muss eine Rotation des OH-Radikals stattfinden, bis zu der Position, in der das Sauerstoffatom eins der Kohlenstoffatome des ungesättigten Kohlenwasserstoffs angreifen kann. Diese Rotation bildet eine Energiebarriere. Im gebundenen Addukt ist dann das ungepaarte Elektron am $\beta$-Kohlenstoffatom lokalisiert. Dabei können sich die betrachteten Reaktionen ganz offensichtlich in der energetischen Position der Barriere relativ zu den Edukten (vgl. Abb. 5.9)unterscheiden. Die Temperaturabhängigkeit der Geschwindigkeitskonstanten wird also in ganz entscheidender Weise durch die Höhe der Barriere beeinflusst.

3. Besitzt einer der Reaktanden mehrere tiefliegende elektronische Zustände oder Potentialflächen, wovon nur einer bzw. eine zur Reaktion beiträgt, so könnte ihre temperaturabhängige Population eine Erklärung für eine mögliche negative Temperaturabhängigkeit der Geschwindigkeitskonstanten sein. Ab der Temperatur, ab der nur noch das unterste elektronische Niveau signifikant besetzt ist, steigt die Geschwindigkeitskonstante nicht weiter an, sondern bleibt konstant. Durch die Spin-Bahn-Kopplung verfügt das Hydroxylradikal über verschiedene elektronische Zustände, der Energieunterschied zwischen den beiden untersten Zuständen beträgt ca. $140 \mathrm{~cm}^{-1}$ [45]. Somit sollten beide Zustände bei Raumtemperatur und darunter besetzt sein.

Wird also für die in dieser Arbeit untersuchten Reaktionen des Hydroxylradikals mit ungesättigten Kohlenwasserstoffen ein schematisches Potential wie in Abb. 5.9 angenommen, so können die verschiedenen Temperaturabhängigkeiten abgeleitet werden.

Zunächst unterscheidet sich die Reaktion des OH-Radikals mit Ethin von der des Ethens unterhalb von $300 \mathrm{~K}$ in der Tatsache, dass die OH-Ethin-Reaktion nahezu temperaturunabhängig scheint, während die $\mathrm{OH}-E$ then-Reaktion eine deutlich negative Abhängigkeit von der Temperatur zeigt. Im Fall des Ethins liegt die Barriere zum gebundenen Addukt deutlich oberhalb der Energie der Edukte (durchgezogene Linie in Abb. 5.9), die Aktivierungsenergie liegt bei ca. $1.6 \mathrm{kcal} / \mathrm{mol}$. Bei genügend großen Energien ist eine Überquerung der Barriere zum gebundenen Addukt ohne weiteres möglich. Bei sinkender Temperatur (kleineren Energien) wird die Barriere weniger leicht überwunden, vielmehr wird in vielen Fällen der $\pi$-Komplex stabilisiert. Somit verläuft die Reaktion des Ethin mit $\mathrm{OH}$ wahrscheinlich zunächst über den langreichweitigen Einfang der Reaktanden zum $\pi$-Komplex, der ohne Aktivierungsenergie gebildet werden kann. Der Einfluss dieser beiden Potentialeigenschaften sowie der unter Punkt 3 beschriebene Effekt der tiefliegenden elektronischen 


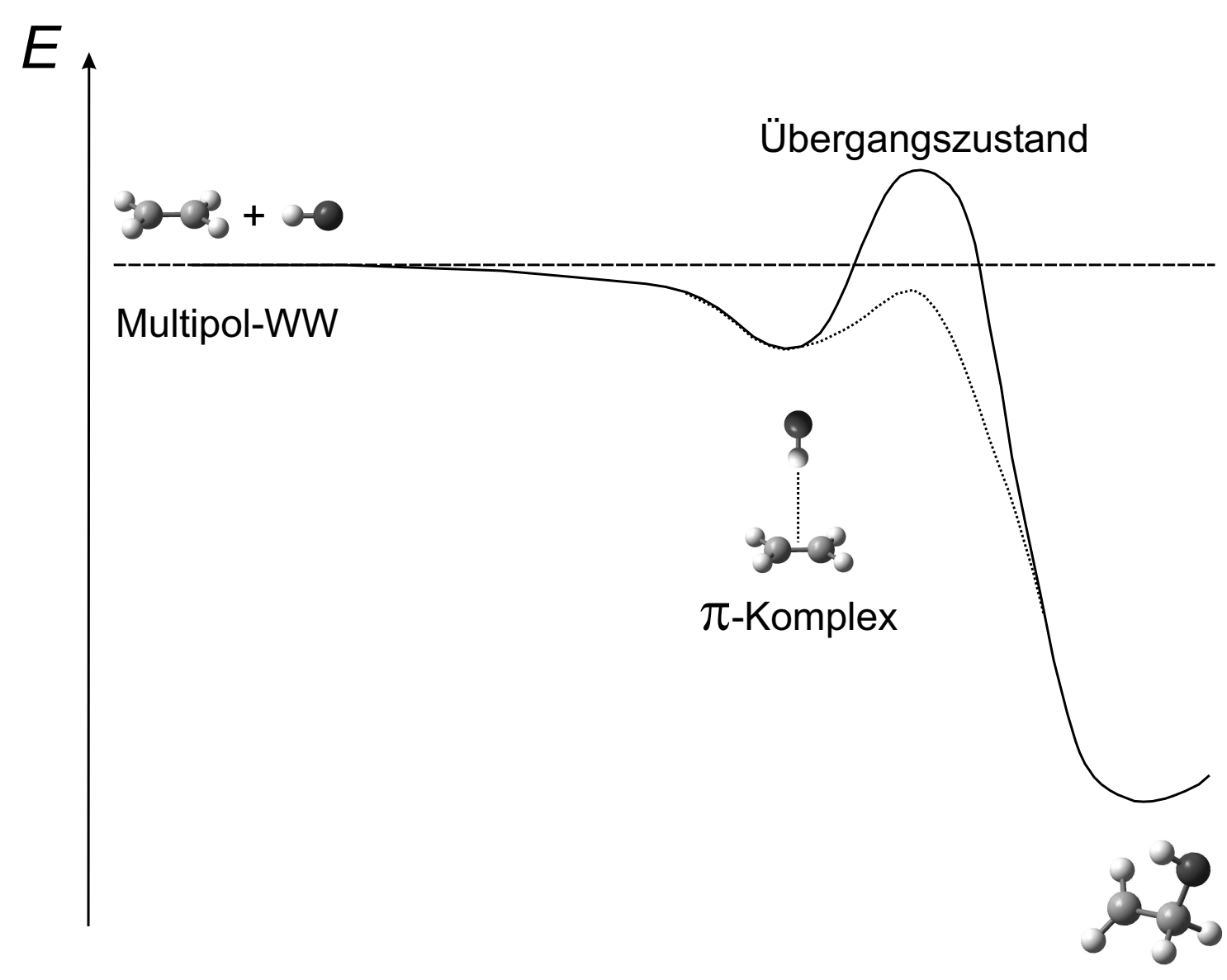

Addukt

Abbildung 5.9: Schematischer Verlauf der potentiellen Energie der Rekombination von Hydroxylradikalen mit ungesättigten Kohlenwasserstoffen

Zustände sollten also zu einer negativen Temperaturabhängigkeit der Geschwindigkeitskonstanten führen, während die ausgeprägte Barriere zur Adduktbildung die Reaktionsgeschwindigkeit zu tiefen Temperaturen hin verlangsamt. Unterhalb von $300 \mathrm{~K}$ scheint der Einfluss der langreichweitigen elektrostatischen Kräfte sowie die Stabilisierung des Komplexes zuzunehmen, die aufgeführten Effekte auf die Temperaturabhängigkeit der Geschwindigkeitkonstanten scheinen sich in etwa aufzuheben, so dass die Geschwindigkeit der Reaktion unterhalb von $300 \mathrm{~K}$ nahezu konstant ist.

Die Reaktion des Ethens mit OH scheint dahingegen eher auf einem in Abb. 5.9 als gepunktete Linie dargestellten Potential abzulaufen. Nach allen bisherigen ex- 
perimentellen und theoretischen Bestimmungen liegt die Barriere zum gebundenen Addukt etwas unterhalb der asymptotischen Energie der Edukte. Da sich die Barriere also unterhalb der Energie der Edukte befindet, wird die Geschwindigkeit der Reaktion lediglich durch die in den Punkten 1, 2 und 3 aufgeführten Effekte bestimmt und nimmt deshalb mit sinkender Temperatur zu.

Somit kann eine grundsätzliche Unterscheidung der Reaktionen des Ethins und Ethens getroffen werden, da sich sowohl die Absolutwerte der gemessenen Geschwindigkeitskonstanten um fast eine Größenordnung unterscheiden als auch die Eigenschaften der Barriere (Position bzw. energetische Lage) deutlich voneinander abweichen. Die Unterschiede in den Reaktionen liegen auf der Hand, auf der einen Seite handelt es sich bei dem Reaktanden um ein doppelt, bei dem anderen um ein dreifach gebundenen Kohlenwasserstoff. Da bei der Addition eines Hydroxylradikals an einen ungesättigten Kohlenwasserstoff eine der Kohlenstoff-Kohlenstoff-Bindung gebrochen werden muss, ist die unterschiedliche Bindungsstärke der Doppel- bzw. Dreifachbindung möglicherweise eine Erklärung für die unterschiedlichen kinetischen Verhalten des Ethins bzw. Ethens. Aufgrund der deutlich höheren Bindungsenergie des Ethins (ca. $123 \mathrm{kcal} / \mathrm{mol}$ ) gegenüber der des Ethens (ca. $100 \mathrm{kcal} / \mathrm{mol}$ ), die sich auch in den Bindungslängen wiederspiegelt (Ethin: $1.2 \AA$, Ethen: $1.3 \AA$ ), scheint eine Adduktbildung aus Ethen und $\mathrm{OH}$ erleichtert.

Durch die unterschiedliche Hybridisierung der Kohlenstoffatome (Ethin: sp, Ethen: $\mathrm{sp}^{2}$ ) ergeben sich ebenfalls abweichende Aziditäten der Kohlenwasserstoffe. Eine mögliche Konsequenz für die Reaktionen des OH-Radikals mit Ethin bzw. Ethen betrifft die Bildung des in mehreren Arbeiten postulierten $\pi$-Komplexes. In diesem $\pi$-Komplex ist das Hydroxylradikal senkrecht mit dem Wasserstoffatom zur Doppelbzw. Dreifachbindung des ungesättigten Kohlenwasserstoffs ausgerichtet. Deswegen liegt die Vermutung nahe, dass der $\pi$-Komplex aufgrund der erhöhten Elektronegativität der C-C-Bindung im Ethin leichter gebildet werden könnte als im Ethen. Weiterhin könnten die unterschiedlichen Elektronegativitäten der Kohlenstoffatome in doppelt oder dreifach gebundenen Kohlenwasserstoffen ein Hinweis auf die Ursache der unterschiedlichen Barrieren für die Reaktion des Hydroxylradikals mit Ethin bzw. Ethen geben. Da sich für die Bildung des Adduktes das Sauerstoffatom des OH-Radikals an eines der Kohlenstoffatome orientieren muss, ist diese Annäherung im Fall des Ethens (weniger elektronegatives Kohlenstoffatom) gegenüber dem Ethin mit einem geringeren energetischen Aufwand durchführbar.

Prinzipell können für die Reaktionen des Propens und Isoprens mit Hydroxylradikalen ähnliche Effekte wie für die entsprechende Reaktion des Ethens auftreten, da deren Barrieren ebenfalls wenige kcal/mol unterhalb der Energie der Edukte liegen. Der Anstieg der Geschwindigkeitskonstanten unterhalb von $300 \mathrm{~K}$ für beide 
Reaktionen (vgl. Abb. 5.7) lässt sich also analog der Reaktion des Ethens erklären. Jedoch muss ein Unterschied zwischen diesen Reaktionen vorliegen, da die Geschwindigkeitskonstante für die OH-Propen-Reaktion anscheinend einem Grenzwert entgegenstrebt, während sie für die OH-Isopren-Reaktion unterhalb von $100 \mathrm{~K}$ sogar abfällt. Durch die Asymmetrie der Moleküle Propen und Isopren gibt es für das Hydroxylradikal unterschiedliche Angriffspositionen an der bzw. den Doppelbindungen, die zu unterschiedlichen Isomeren führen (vgl. Abb. 1.3 und 1.4). Dabei weisen die unterschiedlichen Isomere wahrscheinlich unterschiedliche Barrieren zur Adduktbildung auf. Bei genügend großer Energie können möglicherweise alle Isomere zur Reaktion beitragen, da die energetisch etwas ungünstigeren Addukte isomerisieren können. Deshalb sollte es etwa bei Raumtemperatur und knapp darunter keine Rolle spielen, an welcher Position der Angriff des Hydroxylradikals stattfindet. Bei geringeren Temperaturen und damit kleineren thermischen Energien ist es den energetisch etwas ungünstigeren Isomeren vielleicht nicht mehr möglich, zu den stabilsten Addukten zu isomerisieren, möglicherweise zerfallen sie wieder zu den Edukten. Dies könnte ein Absinken der Geschwindigkeitskonstanten zu tiefen Temperaturen zur Folge haben.

Die Reaktionen der verschiedenen Buten-Isomere (z. B. cis-2-Buten in Abb. 5.7) mit Hydroxylradikalen verlaufen unterhalb von $300 \mathrm{~K}$ mit sinkender Temperatur zunehmend schneller, die gemessenen Geschwindigkeitskonstanten erreichen jedoch ab ca. $70 \mathrm{~K}$ einen Grenzwert von ca. $3 \cdot 10^{-10} \mathrm{~cm}^{-3} \mathrm{~s}^{-1}$. Eine Erklärung laut Punkt 3 scheint hier plausibel. Die Limitierung der Reaktionsgeschwindigkeit zu tiefen Temperaturen könnte aber auch durch die Anzahl der Stöße gegeben sein, da der erreichte Grenzwert im Bereich gaskinetischer Stoßzahlen liegt. Da es sich bei cis- und trans-2-Buten um symmetrische Alkene handelt, ist die Bildung mehrerer isomerer OH-Buten-Addukte unwahrscheinlich. Deshalb wird nach der in dieser Arbeit vorgeschlagenen Hypothese kein Absinken der Geschwindigkeitskonstanten erwartet. Bei der Reaktion des 1-Buten wäre prinzipiell die Ausbildung zweier Hydroxybutenylradikale denkbar, jedoch finden sich solche Überlegungen in Ref. [146] nicht.

Weiterhin lassen sich die in dieser Arbeit bestimmten Geschwindigkeitskonstanten für die Addition des OH-Radikals an ungesättigte Kohlenwasserstoffe bei tiefen Temperaturen mit entsprechenden Arbeiten zu Reaktionen anderer Radikale mit ungesättigten Kohlenwasserstoffen vergleichen. Sowohl die Reaktionen von Ethin mit Cyano(CN)- [143], Methylidin(CH)- [152] und Ethinyl( $\left.\mathrm{C}_{2} \mathrm{H}\right)$-Radikalen $[163,165,154]$ als auch mit Kohlenstoffatomen $\left(\mathrm{C}\left({ }^{3} \mathrm{P}\right)\right)[155]$ wurden in verschiedenen CRESU-Apparaturen bei tiefen Temperaturen untersucht. Alle diese Reaktionen zeigen eine sehr ähnliche Temperaturabhängigkeit der Geschwindigkeitskonstanten, jedoch sind sie wesentlich schneller (Geschwindigkeitskonstanten zwischen 2 und $\left.4 \cdot 10^{-10} \mathrm{~cm}^{3} \cdot \mathrm{s}^{-1}\right)$ als die entsprechende Reaktion mit $\mathrm{OH}\left(k \approx 2 \cdot 10^{-12} \mathrm{~cm}^{3} \cdot \mathrm{s}^{-1}\right)$. 
Damit erreichen die Geschwindigkeitskonstanten schon fast Werte der einfachen Stoßtheorie, d. h. fast jeder Stoß führt zur Reaktion. Die Reaktionen des CN-, CHund des $\mathrm{C}_{2} \mathrm{H}$-Radikals sowie des Kohlenstoffatoms $\left(\mathrm{C}\left({ }^{3} \mathrm{P}\right)\right)$ mit Ethin weisen unterhalb von $300 \mathrm{~K}$ zunächst eine negative Temperaturabhängigkeit auf. Nach einem für die verschiedenen Radikale unterschiedlich positionierten bzw. ausgeprägten Maximum fallen jedoch zu noch tieferen Temperaturen alle Geschwindigkeitskonstanten ab. Das Zustandekommen dieses Maximums wird theoretisch in den entsprechenden Arbeiten mit der sogenannten adiabatic capture theory von Clary [199,200] behandelt, in der die Reaktion als Einfang der Reaktanden auf einem langreichweitigen Potential ohne Barriere beschrieben wird.

Diese Eigenschaften des Potentials der Reaktionskoordinate sind vor allem für IonMolekül-Reaktionen bekannt, die adiabatic capture theory wurde deshalb für solche Reaktionen entwickelt, später aber auch auf andere Reaktionstypen (RadikalRadikal-, Radikal-Molekül-Reaktionen) angewendet. Dabei werden die berechneten Geschwindigkeitskonstanten als oberer Grenzwert für die tatsächlichen Werte angesehen und sollen genauer sein, je tiefer die Temperatur ist. Das elektrostatische Wechselwirkungspotential zwischen den Molekülschwerpunkten wird im Fall der Reaktion zweier Neutralteilchen durch Dipol-Dipol bzw. Dipol-Quadrupol-Kräfte beschrieben, Schwingungen der beteiligten Moleküle werden vernachlässigt. Für die Reaktion zwischen polaren Radikalen im ${ }^{2} \Pi$-Zustand und Molekülen im ${ }^{1} \Sigma$-Zustand sagt die adiabatic capture theory ein Maximum in der Temperaturabhängigkeit der Geschwindigkeitskonstanten voraus, welches z. B. für die Reaktion der kohlenstoffhaltigen Radikale mit ungesättigten Kohlenwasserstoffen beobachtet wird. Dabei wird die Lage des Maximums $T_{\max }$ nach Gl. 5.2 vom Dipol- $\mu$ und Quadrupolmoment $Q$ sowie der Rotationskonstante $B$ des Moleküls im ${ }^{1} \Sigma$-Zustand, in diesem Fall also des Ethins, bestimmt.

$$
T_{\max }=a \mu^{b} Q^{c} B^{d}
$$

$a, b, c$ und $d$ sind Anpassungsparameter. Somit ist die Vorhersage der Maxima in der Temperaturabhängigkeit der Geschwindigkeitskonstanten möglich, vorausgesetzt, das Molekül besitzt ein Dipol- bzw. Quadrupolmoment. Die Ausbildung eines Maximums für die Reaktion des Ethins mit OH-Radikalen ist nach Gl. 5.2 also nicht zu erwarten.

Die Addition anderer Radikale an das Ethen wurde bereits bei tiefen Temperaturen in CRESU-Apparturen untersucht. Die Temperaturabhängigkeit der Reaktionen des Cyano(CN)- [143], Methylidin $(\mathrm{CH})$ - [152] und des Ethinyl $\left(\mathrm{C}_{2} \mathrm{H}\right)$-Radikals [78] als auch des Kohlenstoffatoms $\left(\mathrm{C}\left({ }^{3} \mathrm{P}\right)\right)$ [155] mit Ethen verhält sich ähnlich den analogen Reaktionen der Radikale mit Ethin. Auch in diesem Fall ist ein Maximum in der Temperaturabhängigkeit erkennbar, die Geschwindigkeitskonstanten 
liegen ebenfalls in einem Bereich von 2 bis $5 \cdot 10^{-10} \mathrm{~cm}^{3} \cdot \mathrm{s}^{-1}$ und sind damit ca. eineinhalb Größenordnungen größer als die entsprechende Reaktion des OH-Radikals. Auch für die Reaktionen der verschiedenen Radikale $\left(\mathrm{CN}, \mathrm{CH}, \mathrm{C}_{2} \mathrm{H}\right.$ und $\mathrm{C}\left({ }^{3} \mathrm{P}\right)$ ) mit Ethen wird der Einfang der Reaktanden auf einem langreichweitigen Potential ohne Barriere durch elektrostatische Anziehungskräfte als Erklärung für die beobachtete Temperaturabhängigkeit herangezogen. Obwohl also die Reaktionen der nicht-OH-Radikale ebenso wie die Reaktion des OH-Radikals mit Ethen nach bisherigem Erkenntnisstand ohne Barriere ablaufen, weisen die entsprechenden Geschwindigkeitskonstanten bei tiefen Temperaturen den oben beschriebenen großen Unterschied (ca. Faktor 1.5) auf.

Ein prinzipieller Unterschied der Reaktionen des Ethens mit kohlenstoffhaltigen Radikalen bzw. dem OH-Radikal könnte die Stabilität der zunächst entstehenden Radikal-Ethen-Addukte zu sein. Während das Hydroxyethenylradikal mit einer Energie von ca. $26 \mathrm{kcal} / \mathrm{mol}$ als verhältnismäßig stabil angesehen werden kann, wird die Stabilsierung eines solchen Adduktes z. B. für die Reaktion des CH-Radikals mit Ethen ausgeschlossen. Nach der Anlagerung des CH-Radikals an das Ethen (Komplexbildung) findet vielmehr eine sofortige Fragmentierung des angeregten Adduktes statt, dabei wird ein Wasserstoffatom abstrahiert. Analog verläuft auch die Reaktion des Kohlenstoffatoms mit Ethen über eine Addition und anschließende Eliminierung eines Wasserstoffatoms, für die Reaktion des CN-Radikals mit Ethen wird die direkte Bildung von HCN durch Wasserstoffabstraktion favorisiert. Durch die Kurzlebigkeit der möglicherweise gebildeten intermediären Komplexe zeigen die Geschwindigkeitskonstanten der Reaktionen von CN-, CH- und $\mathrm{C}_{2} \mathrm{H}-\mathrm{Radikalen} \mathrm{so-}$ wie des Kohlenstoffatoms keine Druckabhängigkeit. Aufgrund der aufgezeigten Unterschiede in der Stabilität möglicher Intermediate und dem daraus resultierenden großen Unterschied der Absolutwerte der Geschwindigkeitskonstanten ist eine Analogie zwischen den Reaktionen der kohlenstoffhaltigen Radikale bzw. dem Hydroxylradikal mit Ethen nur bedingt gegeben. Gemeinsam haben alle diese Reaktionen eine negative Temperaturabhängigkeit, im Fall der kohlenstoffhaltigen Radikale sinken die Geschwindigkeitskonstanten nach dem Durchlaufen eines Maximums zu noch tieferen Temperaturen wieder ab. Ob sich die Reaktion von OH-Radikalen mit Ethen unterhalb von $60 \mathrm{~K}$ möglicherweise auch so verhält, müssen zukünftige Messungen zeigen.

Da die Reaktion des OH-Radikals mit Isopren ein Maximum in der Temperaturabhängigkeit der Geschwindigkeitskonstanten aufweist, bietet sich eine entsprechende Vorhersage des Maximums für die OH-Isopren-Reaktion durch Gl. 5.2 an. Eine Prognose anhand des Dipol- und Quadrupolmoments sowie der Rotationskonstante des Isoprens ergibt sich nach Gl. 5.2 ein Maximum bei unter $10 \mathrm{~K}$. Diese Vorhersage steht im starken Gegensatz zum experimentell bestimmten Maximum bei ca. $100 \mathrm{~K}$. 
Somit scheint das Absinken der Geschwindigkeitskonstanten zu noch tieferen Temperaturen andere Ursachen zu haben.

Die im Rahmen der vorliegenden Arbeit durchgeführten Untersuchungen zeigen, dass temperaturabhängige Messungen von Geschwindigkeitskonstanten bei tiefen Temperaturen zum Verständnis des Reaktionsmechanismus sowie der Energetik der Reaktion beitragen können. Dabei stellt sich heraus, dass kleine Unterschiede in den Potentialeigenschaften in chemische relevanten Energiebereichen gerade bei tiefen Temperaturen einen bemerkenswerten Einfluss auf die Temperaturabhängigkeit und die Absolutwerte der Geschwindigkeitskonstanten haben können. 


\section{Kapitel 6}

\section{Ausblick}

Zunächst ist geplant, die Bestimmung von Geschwindigkeitskonstanten in der Göttinger CRESU-Apparatur bei noch tieferen Temperaturen durchzuführen. Die Abkühlung zu noch tieferen als den bisher erreichten Temperaturen lassen sich einerseits durch die Verwendung anderer Trägergase mit größeren Adiabatenkoeffizienten wie Helium oder Argon erreichen. Andererseits ist es möglich, das Trägergas bereits vor der Expansion sowie während der Expansion vorzukühlen. Mit der Kombination dieser beiden Methoden konnte eine tiefste Temperatur von $8 \mathrm{~K}$ in einer CRESUApparatur erreicht werden. Diese für kinetische Untersuchungen extrem tiefe Temperaturen könnten auch für die in dieser Arbeit untersuchten Reaktionen weiteren Aufschluss über Mechanismus und Energetik der Reaktionen geben.

Auf theoretischem Gebiet wäre der Vergleich mit Rechnungen denkbar, die sich nicht auf das SAC-Modell berufen. Denn in den hier durchgeführten SACM-Rechnungen werden sämtliche in Abb. 5.9 aufgeführten Eigenschaften der Potentialfläche (langreichweitige Multipol-Wechselwirkungen, $\pi$-Komplex, Barriere) missachtet. Die offensichtlich für die Addition von Hydroxylradikalen an Ethin vorhandene Reaktionsbarriere lässt eine Berechnung nach dem RRKM-Modell sinnvoll erscheinen, da in diesem explizit die Position und die Eigenschaften des Übergangszustandes bekannt sein müssen. Die Eigenschaften des Übergangszustandes ließen sich, wie bereits in dieser Arbeit für die Edukte und Produkte durchgeführt, mit modernen quantenchemischen Methoden berechnen.

In allen kinetischen Untersuchungen in den verschiedenen CRESU-Apparaturen wurden fast ausschließlich Reaktionen anhand von lichtemittierenden Molekülen wie dem Hydroxyl- $(\mathrm{OH})$, Cyano $(\mathrm{CN})$-, Methylidin $(\mathrm{CH})$-, Ethinyl $\left(\mathrm{C}_{2} \mathrm{H}\right)$-Radikalen als auch dem Kohlenstoffatom $\left(\mathrm{C}\left({ }^{3} \mathrm{P}\right)\right)$ untersucht. Dabei wurde jeweils die Konzentration dieser in der Minderheit vorliegenden Spezies zeitlich durch Laseranregung und nachfolgende Detektion der Fluoreszenz (LIF für laserinduzierte Fluorezenz) verfolgt. Die LIF ist experimentell wenig aufwendig und wurde erfolgreich für die 
Beobachtung der $\mathrm{OH}-$, CN- und $\mathrm{CH}-$ Radikale angewendet. Die $\mathrm{C}_{2} \mathrm{H}$-Radikale wurden über die Chemilumineszenz des elektronisch angeregten $\mathrm{CH}\left(\mathrm{A}^{2} \Delta\right)$, welches in der Reaktion von $\mathrm{C}_{2} \mathrm{H}$ und $\mathrm{O}_{2}$ gebildet wird, detektiert. Kohlenstoffatome wurden über die Chemilumineszenz des bei der Reaktion von $\mathrm{C}\left({ }^{3} \mathrm{P}\right)$ mit $\mathrm{NO}_{2}$ entstehenden NO beobachtet. Eine Ausnahme bildet die Studie von Leone et al. zur Reaktion des Ethinyl $\left(\mathrm{C}_{2} \mathrm{H}\right)$-Radikals, in der - wie in den hauptsächlich in Rennes durchgeführten Arbeiten zu Ion-Molekül-Reaktionen - ein Massenspektrometer zur Detektion von Reaktanden sowie Produkten eingesetzt wurde. Der Einsatz eines Massenspektrometers in der Göttinger CRESU-Apparatur ist zur zeitaufgelösten Detektion verschiedener Reaktionsprodukte geplant. Eine Weiterentwicklung von Detektionstechniken für die CRESU-Technik wäre die Detektion der an einer Reaktion beteiligten nichtfluoreszeierender Spezies mit Hilfe der Cavity-Ringdown-Spektroskopie (CRDS), die bereits in Göttingen etabliert wurde [201,202]. Die Cavity-RingdownSpektroskopie ist eine hochempfindliche Absorptionsspektroskopie und eignet sich damit hervorragend zur Detektion von Radikalen in kleinen Konzentrationen. Auf diese Weise ist z. B. die Untersuchung der atmosphärenchemisch äußerst bedeutenden Rekombination von molekularem mit atomaren Sauerstoff zu Ozon bei tiefen Temperaturen möglich. So ließe sich der Bereich der mit der CRESU-Technik untersuchten Reaktionen wesentlich erweitern.

Eine weitere interessante Kombination der CRESU-Technik ist mit der Methode der kinetisch kontrollierten selektiven Ionisation (KCSI) möglich. Mit dieser Kombination wäre es möglich, die Eigenschaften von Stoßprozessen in bestimmten Energiefenstern der Moleküle im Bereich hoher Zustandsdichten bei tiefen Temperaturen zu untersuchen. Aus KCSI-Experimenten lässt sich durch eine Mastergleichungsanalyse die Energieabhängigkeit der Stoßübergangswahrscheinlichkeit bestimmmen. Daraus ergeben sich dann die mittlere pro Stoß übertragene Energie sowie die Breite der Verteilung. Alternativ zur Detektion der ionisierten Spezies könnte der Nachweis der stoßrelaxierten Moleküle auch durch Fluoreszenz erfolgen (KCSF).

In einigen Messungen in der Göttinger CRESU-Apparatur konnte ein ungewöhnlicher Effekt in der Konzentrationsabhängigkeit der bestimmten Geschwindigkeitskonstanten pseudo-erster Ordnung festgestellt werden. Während sich im allgemeinen eine lineare Abhängigkeit der bestimmten Geschwindigkeitskonstanten pseudoerster Ordnung von der Konzentration des Reaktionspartners (ungesättigter Kohlenwasserstoff) ergab, traten im Fall der Reaktion des Hydroxylradikals mit Isopren und in kürzlich durchgeführten Messungen zur entsprechenden Reaktion des Benzols Abweichungen zu dieser linearen Abhängigkeit auf. Bei kleinen Reaktandkonzentrationen ließ sich zunächst ein linearer Verlauf beobachten, nach Durchlaufen eines Maximums sanken die Geschwindigkeitskonstanten zu größeren Konzentrationen jedoch wieder ab. Ähnliche Effekt wurden bereits in den Reaktionen des 
CN-Radikals mit ungesättigten Kohlenwasserstoffen beobachtet und dort als die Bildung von Molekülclustern bei tiefen Temperaturen und hohen Reaktandkonzentrationen interpretiert [143]. Tatsächlich tritt der oben beschriebene Effekt nicht in allen Fällen auf, sondern nur, wenn eine bestimmte Konzentration an Isopren bzw. Benzol überschritten war und die Temperatur tief genug war, konnten die Abweichungen beobachtet werden. Die Frage, um welche Art von Clustern es sich handelt, konnte im Rahmen dieser Arbeit nicht geklärt werden. Es liegt jedoch die Vermutung nahe, dass es sich dabei zumindest zu einem gewissen Teil um Isopren- bzw. Benzoldimere handelt. Denn es wurde kürzlich in der CRESU-Apparatur in Rennes die Benzol-Dimerisierung anhand der Fluoreszenz des Monomers in einer Lavaldüsenexpansion untersucht [172]. Somit ergibt sich hier eine weitere Anwendung der CRESU-Technik, die Untersuchung der Kinetik der Clusterbildung. 



\section{Anhang A}

\section{Geometrien, Frequenzen und Energien}

In den folgenden Tabellen finden sich die optimierten Geometrien, Frequenzen und die Energien der beteiligten Spezies, die mit Hilfe verschiedener quantenchemischer Methoden und verschiedener Basissätze (Gaussian98 [203]) berechnet wurden. Dabei wurde zu Beginn eine Geometrieoptimierung anhand der Energien der beteiligten Moleküle durchgeführt, anschließend wurden die Frequenzen der Normalmoden berechnet. Zur Verkürzung der Rechenzeit wurde zunächst die HartreeFock-Methode mit einem moderaten Basissatz benutzt, später dann die Dichtefunktionaltheorie (B3LYP) und zum weiteren Vergleich auch verschiedene Stufen der störungstheoretischen Ansätze $\left(\mathrm{MP}_{n}\right)$. Die nachfolgenden Tabellen werden jeweils kurz diskutiert, ein Vergleich der berechneten Molekülparameter wurde, soweit vorhanden, sowohl mit experimentellen Werten als auch mit anderen theoretischen Arbeiten gezogen. Dabei ist zu beachten, dass die beteiligten Radikale $\left(\mathrm{OH}, \mathrm{C}_{2} \mathrm{H}_{2} \mathrm{OH}\right.$ und $\left.\mathrm{C}_{2} \mathrm{H}_{4} \mathrm{OH}\right)$ mit einem ,open shell“-, die ungesättigten Kohlenwasserstoffe $\left(\mathrm{C}_{2} \mathrm{H}_{2}\right.$ und $\left.\mathrm{C}_{2} \mathrm{H}_{4}\right)$ mit einem , closed shell"-Modell behandelt wurden. Für Moleküle, die eine gerade Anzahl an Elektronen und deswegen nur Elektronenpaare entgegengesetzten Spins besitzen (abgeschlossene Schalen), wird deswegen das „,closed shell"- oder auch ,spin restricted“-Modell verwendet, die Elektronenpaare werden in ein Orbital gezwungen. Im Gegensatz dazu steht das ,open shell“- oder auch ,spin unrestricted“-Modell, nach dem Elektronen unterschiedlichen Spins unterschiedliche Orbitale besetzen. Es wird für Moleküle mit ungerader Elektronenanzahl (z. B. Ionen), angeregte Zustände oder Zustände mit sonstiger ungewöhnlicher Elektronenkonfiguration benutzt.

Alle Berechnungen der Moleküle wurden jeweils im elektronischen Grundzustand durchgeführt. 


\section{A.1 Hydroxylradikal}

\section{A.1.1 Geometrie}

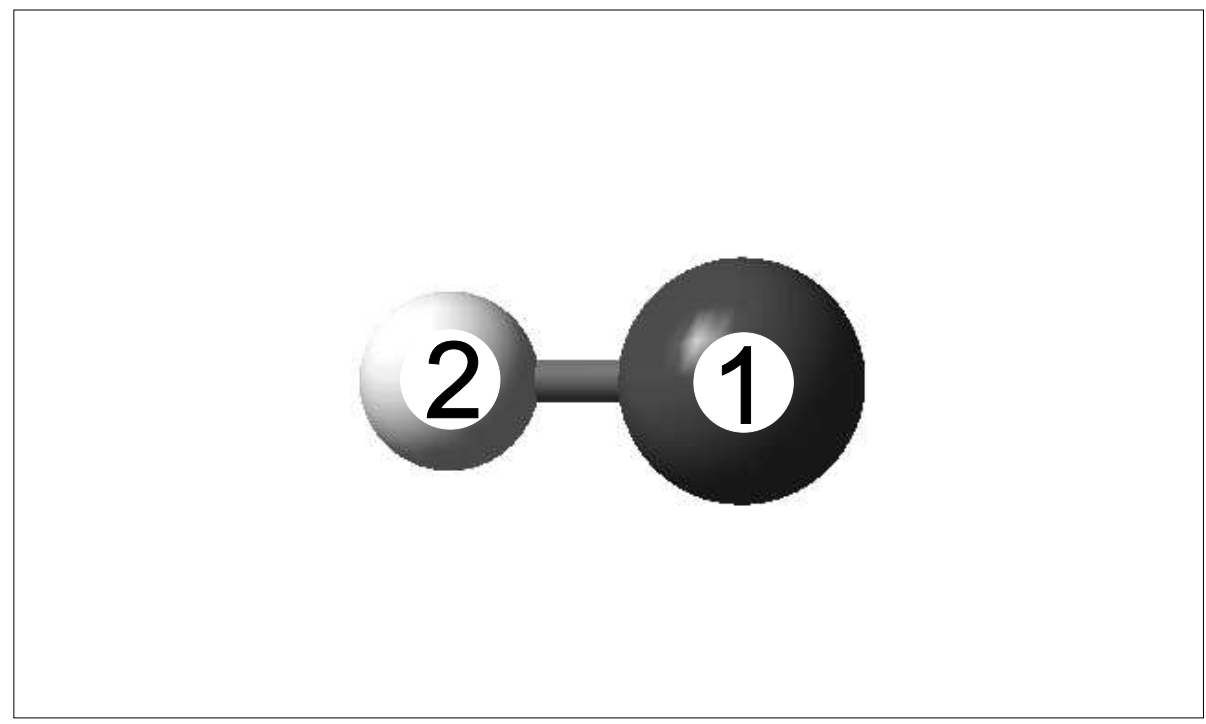

Abbildung A.1: Strukturformel des Hydroxylradikals

\begin{tabular}{|cc|ll|lll|}
\hline $\begin{array}{c}\text { Atom. } \\
\text { Nr. }\end{array}$ & $\begin{array}{c}\text { Chem. } \\
\text { Symb. }\end{array}$ & $\begin{array}{l}\text { Abstand } \\
\text { in }[\AA]\end{array}$ & Winkel Diederw. & $\mathrm{A}^{(1)}$ & $\mathrm{B}^{(2)}$ & $\mathrm{C}^{(3)}$ \\
\hline \hline 1 & $\mathrm{O}$ & & & & \\
2 & $\mathrm{H}$ & 0.9742 & & 1 & \\
\hline
\end{tabular}

Tabelle A.1: Mit (B3LYP-6-311++G(3df,3pd)) berechnete Gleichgewichtsgeometrie des Hydroxylradikals $(\mathrm{OH})$. (1)Abstand des Atoms I zu Atom A; (2) Winkel zwischen Atom I, Atom A und Atom B; (3) Winkel der Flächen, die von den Atomen I-A-B und A-B-C aufgespannt werden

\section{A.1.2 Normalmoden}

Die Ergebnisse für die Berechnung der OH-Streckschwingung des Hydroxylradikals zeigen, dass die B3LYP-Methode den besten Wert im Vergleich mit dem Literaturwert liefert (Tab. A.2). Dabei ist zu beachten, dass die aufwendigere Berechnung mit der Störungstheorie vierter Ordnung $\left(\mathrm{MP}_{4}\right)$ keine exakteren Werte liefert 


\begin{tabular}{|c|c|}
\hline $\begin{array}{c}\text { Methode/Basissatz } \\
\text { bzw. Referenz }\end{array}$ & $\nu\left[\mathrm{cm}^{-1}\right]$ \\
\hline \hline $\mathrm{HF} / 6-311++\mathrm{G}(3 \mathrm{df}, 3 \mathrm{pd})$ & 4047 \\
$\mathrm{MP} 4 / 6-311++\mathrm{G}(3 \mathrm{df}, 3 \mathrm{pd})$ & 3790 \\
$\mathrm{~B} 3 \mathrm{LYP} / 6-311++\mathrm{G}(3 \mathrm{df}, 3 \mathrm{pd})$ & 3714 \\
{$[204]$} & 3738 \\
\hline
\end{tabular}

Tabelle A.2: Berechnete Normalmoden des Hydroxylradikals (OH).

als die Dichtefunktionaltheorie, dies wird auch in den Vergleichen der absoluten Energien deutlich (Tab. A.3). Deutlich wird außerdem, dass Berechnungen nach der Hartree-Fock-Methode, auch mit großen Basissätzen, nur als Startpunkt einer quantenchemischen Rechnung dienen sollten.

\section{A.1.3 Energien}

\begin{tabular}{|l|ccc|}
\hline \multirow{2}{*}{} & \multicolumn{3}{|c|}{ Energien in Hartree/Teilchen } \\
& $\mathrm{HF} / \mathrm{B}_{2}$ & $\mathrm{~B} 3 \mathrm{LY} / \mathrm{B}_{2}$ & $\mathrm{MP} 4 / \mathrm{B}_{2}$ \\
\hline \hline $\mathrm{E}+\mathrm{ZPE}$ & -75.410119 & -75.757784 & -75.630548 \\
$\mathrm{E}$ & -75.419339 & -75.766245 & -75.639182 \\
\hline
\end{tabular}

Tabelle A.3: Berechnete elektronische Energien (mit/ohne Nullpunktsenergie) des Hydroxylradikals $(\mathrm{OH})$. 


\section{A.2 Ethin}

\section{A.2.1 Geometrie}

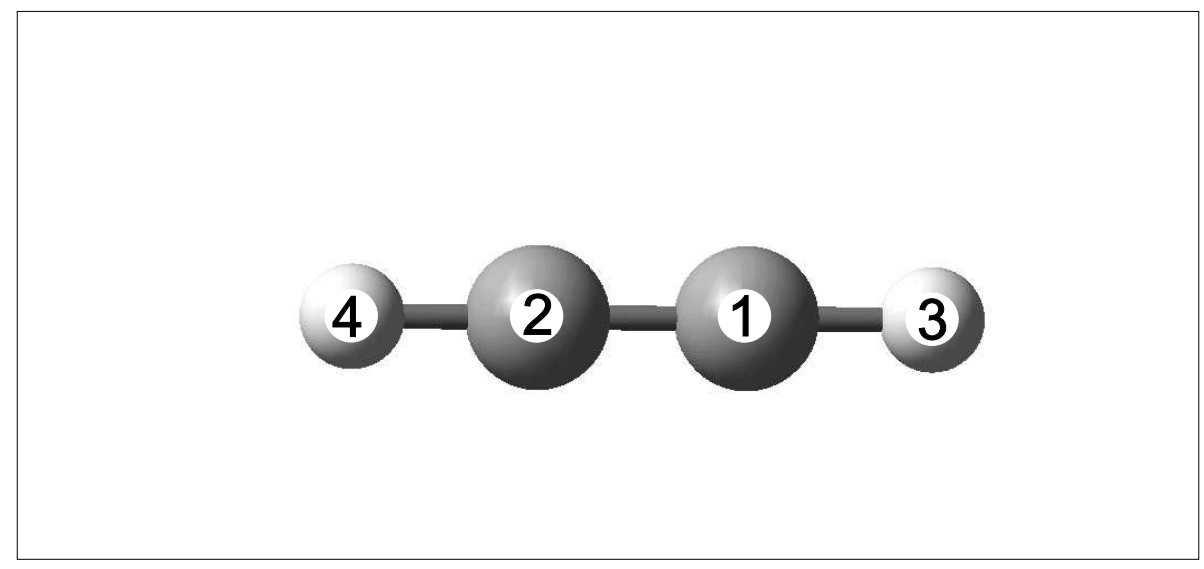

Abbildung A.2: Strukturformel des Ethins $\left(\mathrm{C}_{2} \mathrm{H}_{2}\right)$

\begin{tabular}{|cc|ccc|ccc|}
\hline $\begin{array}{c}\text { Atom. } \\
\text { Nr. }\end{array}$ & $\begin{array}{c}\text { Chem. } \\
\text { Symb. }\end{array}$ & $\begin{array}{l}\text { Abstand } \\
\text { in }[\AA]\end{array}$ & Winkel & Diederw. & $\mathrm{A}^{(1)}$ & $\mathrm{B}^{(2)}$ & $\mathrm{C}^{(3)}$ \\
\hline \hline 1 & $\mathrm{C}$ & & & & & & \\
2 & $\mathrm{C}$ & 1.1963 & & & 1 & & \\
3 & $\mathrm{H}$ & 1.0623 & 180.000 & & 1 & 2 & \\
4 & $\mathrm{H}$ & 1.0623 & 180.000 & & 2 & 1 & \\
\hline
\end{tabular}

Tabelle A.4: Mit (B3LYP-6-311++G(3df,3pd)) berechnete Gleichgewichtsgeometrie des Ethins $\left(\mathrm{C}_{2} \mathrm{H}_{2}\right)$.

\section{A.2.2 Normalmoden}

Bei der Ermittlung der Frequenzen des Ethins zeigt sich ein ähnliches Bild wie im Fall des Hydroxylradikals. Während die nach der HF-Methode berechneten Frequenzen noch ca. $15 \%$ höher als die Literaturwerte liegen, sind es bei der später für die SACM-Rechnungen verwendeten Methode (B3LYP) nur noch ca. 4\% (Tab. A.5). Einen noch deutlicheren Effekt zeigt sich in den berechneten Energien (Tab. A.6). 


\begin{tabular}{|c|c|c|cc|}
\hline $\begin{array}{c}\text { Mode } \\
\text { Nr. }\end{array}$ & Typ & $\begin{array}{c}\text { exp. } \\
{[204]}\end{array}$ & HF $/ B_{1}$ & B3LYP $/ B_{2}$ \\
\hline \hline 1 & CH-bend(a) & 612 & 893 & 657 \\
2 & CH-bend(a) & 612 & 893 & 657 \\
3 & CH-bend(s) & 730 & 900 & 765 \\
4 & CH-bend(s) & 730 & 900 & 765 \\
5 & CC-stretch & 1974 & 2210 & 2066 \\
6 & CH-stretch(a) & 3289 & 3613 & 3407 \\
7 & CH-stretch(s) & 3374 & 3732 & 3509 \\
\hline
\end{tabular}

Tabelle A.5: Berechnete Normalmoden des Ethins $\left(\mathrm{C}_{2} \mathrm{H}_{2}\right)$ in $\mathrm{cm}^{-1}$. Basissätze: $\mathrm{B}_{1}=6-31 \mathrm{G}, \mathrm{B}_{2}=6-311++\mathrm{G}(3 \mathrm{df}, 3 \mathrm{dp})$

\section{A.2.3 Energien}

\begin{tabular}{|l|lc|}
\hline & \multicolumn{2}{|c|}{ Energien in Hartree/Teilchen } \\
& HF $/ B_{1}$ & B3LYP/B \\
& \\
\hline \hline E+ZPE & -76.762826 & -77.335398 \\
$\mathrm{E}$ & -76.792762 & -77.362337 \\
\hline
\end{tabular}

Tabelle A.6: Berechnete elektronische Energien (mit/ohne Nullpunktsenergie) des Ethins $\left(\mathrm{C}_{2} \mathrm{H}_{2}\right)$. 


\section{A.3 Hydroxyethinylradikal}

\section{A.3.1 Geometrie}

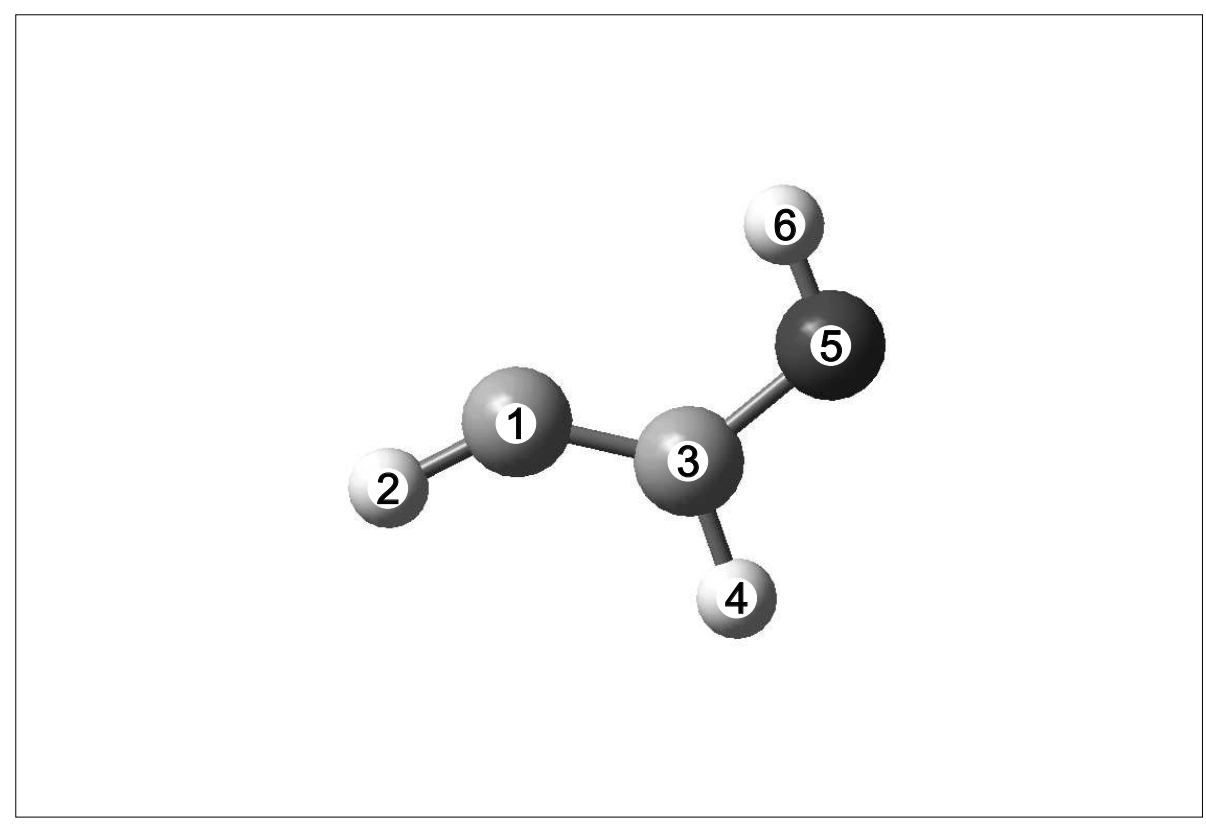

Abbildung A.3: Strukturformel des Hydroxyethinylradikals $\left(\mathrm{C}_{2} \mathrm{H}_{2} \mathrm{OH}\right)$

\begin{tabular}{|cc|lcr|rrr|}
\hline $\begin{array}{c}\text { Atom. } \\
\text { Nr. }\end{array}$ & $\begin{array}{c}\text { Chem. } \\
\text { Symb. }\end{array}$ & $\begin{array}{l}\text { Abstand } \\
\text { in }[\AA]\end{array}$ & Winkel & Diederw. & $\mathrm{A}^{(1)}$ & $\mathrm{B}^{(2)}$ & $\mathrm{C}^{(3)}$ \\
\hline \hline 1 & $\mathrm{C}$ & & & & & & \\
2 & $\mathrm{H}$ & 1.0727 & & & 1 & & \\
3 & $\mathrm{C}$ & 1.3075 & 139.524 & & 1 & 2 & \\
4 & $\mathrm{H}$ & 1.0881 & 121.919 & 0.000 & 3 & 1 & 2 \\
5 & $\mathrm{O}$ & 1.3643 & 127.289 & 180.000 & 3 & 1 & 2 \\
6 & $\mathrm{H}$ & 0.9646 & 108.951 & 180.000 & 5 & 3 & 4 \\
\hline
\end{tabular}

Tabelle A.7: Mit (B3LYP-6-311++G(3df,3pd)) berechnete Gleichgewichtsgeometrie des Hydroxyethinylradikals $\left(\mathrm{C}_{2} \mathrm{H}_{2} \mathrm{OH}\right)$.

Das primäre Addukt der Reaktion eines Hydroxylradikals $(\mathrm{OH})$ mit Ethin $\left(\mathrm{C}_{2} \mathrm{H}_{2}\right)$ ist das Hydroxyethinylradikal $\left(\mathrm{C}_{2} \mathrm{H}_{2} \mathrm{OH}\right)$. Dieses gebundene Addukt ist nach den bisherigen und den in dieser Arbeit durchgeführten Rechnungen planar und durch eine lokalisierte Doppelbindung zwischen den beiden Kohlenstoffatomen charakterisiert 
(Abb. A.3, Tab. A.4). Das freie Elektron befindet sich an dem Kohlenstoffatom, welches dem Hydroxyl-substituierten Kohlenstoffatom benachbart ist, deswegen werden solche Radikale auch als $\beta$-Hydroxyradikale bezeichnet.

\section{A.3.2 Normalmoden}

\begin{tabular}{|c|c|ccc|}
\hline $\begin{array}{c}\text { Mode } \\
\text { Nr. }\end{array}$ & Typ & HF $/ \mathrm{B}_{2}$ & MP2/B & B3LYP $/ \mathrm{B}_{2}$ \\
\hline \hline 1 & OH-torsion & 452 & 488 & 467 \\
2 & CCO-deform. & 538 & 493 & 474 \\
3 & $\mathrm{C}_{1} \mathrm{H}-$ bend & 704 & 706 & 569 \\
4 & $\mathrm{C}_{1} \mathrm{H}-$ bend & 915 & 813 & 769 \\
5 & $\mathrm{C}_{3} \mathrm{H}-$ bend & 916 & 970 & 843 \\
6 & $\mathrm{CO}$-stretch & 1176 & 1124 & 1098 \\
7 & $\mathrm{C}_{3} \mathrm{H}-$ bend & 1342 & 1282 & 1244 \\
8 & OH-bend & 1436 & 1387 & 1355 \\
9 & CC-stretch & 1665 & 1899 & 1667 \\
10 & $\mathrm{C}_{3} \mathrm{H}-$ stretch & 3281 & 3191 & 3107 \\
11 & $\mathrm{C}_{1} \mathrm{H}-$ bend & 3396 & 3366 & 3307 \\
12 & OH-stretch & 4138 & 3833 & 3786 \\
\hline
\end{tabular}

Tabelle A.8: Berechnete Normalmoden des Hydroxyethinylradikals $\left(\mathrm{C}_{2} \mathrm{H}_{2} \mathrm{OH}\right)$ in $\mathrm{cm}^{-1}$. Basissätze: $\mathrm{B}_{3}=6-311++\mathrm{G}(2 \mathrm{~d}, \mathrm{dp}), \mathrm{B}_{2}=6-311++\mathrm{G}(3 \mathrm{df}, 3 \mathrm{dp})$

Die berechneten Frequenzen lassen sich mangels aktueller Literaturdaten nicht vergleichen, der Vergleich der Methoden und Basissätze zeigt, dass die Kombination der Dichtefunktionaltheorie mit genügend großem Basissatz das beste Ergebnis liefert.

\section{A.3.3 Energien}

\begin{tabular}{|l|ccc|}
\hline & \multicolumn{3}{|c|}{ Energien in Hartree/Teilchen } \\
& $\mathrm{HF} / \mathrm{B}_{2}$ & $\mathrm{~B} 3 \mathrm{LYP} / \mathrm{B}_{2}$ & $\mathrm{MP} / \mathrm{B}_{3}$ \\
\hline \hline $\mathrm{E}+\mathrm{ZPE}$ & -152.262693 & -153.144768 & -152.730801 \\
$\mathrm{E}$ & -152.308162 & -153.187336 & -152.783212 \\
\hline
\end{tabular}

Tabelle A.9: Berechnete elektronische Energien (mit/ohne Nullpunktsenergie) des Hydroxyethinylradikals $\left(\mathrm{C}_{2} \mathrm{H}_{2} \mathrm{OH}\right)$. 


\section{A.4 Ethen}

\section{A.4.1 Geometrie}

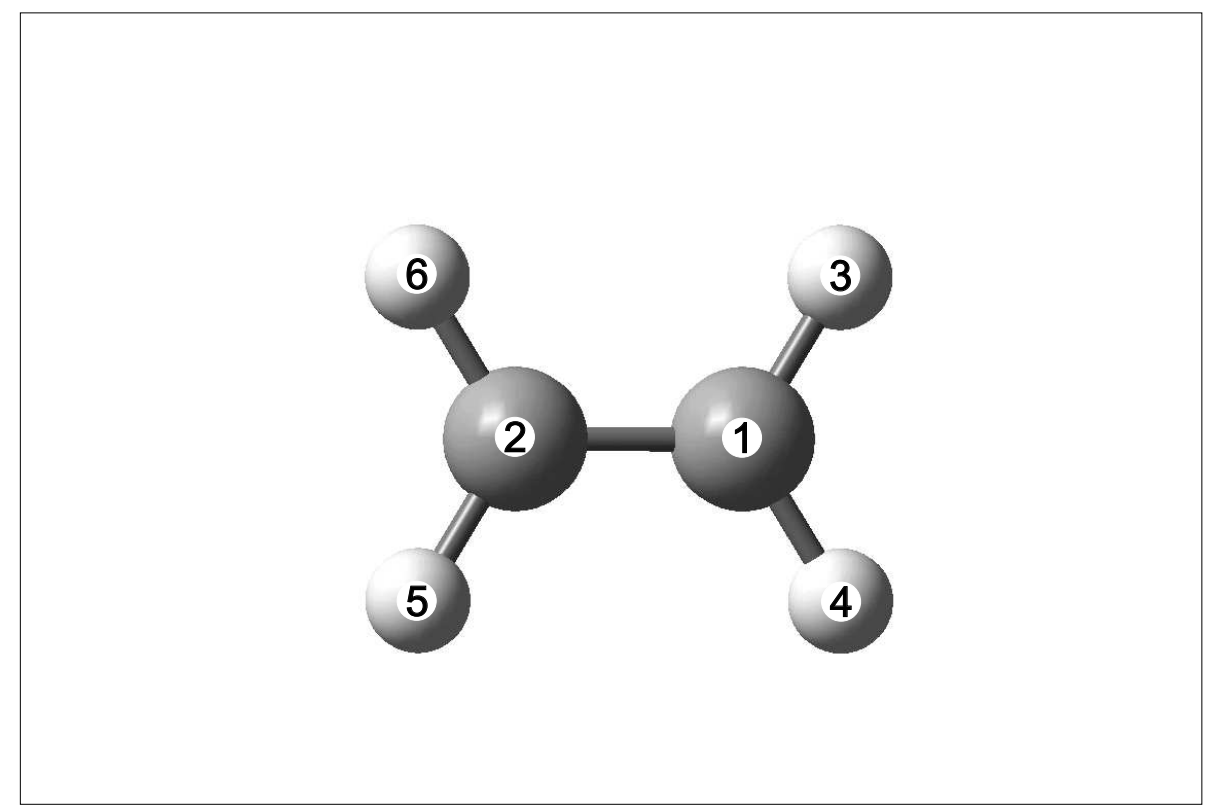

Abbildung A.4: Strukturformel des Ethens $\left(\mathrm{C}_{2} \mathrm{H}_{4}\right)$

\begin{tabular}{|cc|ccc|rrr|}
\hline $\begin{array}{c}\text { Atom. } \\
\text { Nr. }\end{array}$ & $\begin{array}{l}\text { Chem. } \\
\text { Symb. }\end{array}$ & $\begin{array}{l}\text { Abstand } \\
\text { in }[\AA]\end{array}$ & Winkel & Diederw. & $\mathrm{A}^{(1)}$ & $\mathrm{B}^{(2)}$ & $\mathrm{C}^{(3)}$ \\
\hline \hline 1 & $\mathrm{C}$ & & & & & & \\
2 & $\mathrm{C}$ & 1.3247 & & & 1 & & \\
3 & $\mathrm{H}$ & 1.0825 & 121.757 & 180.000 & 1 & 2 & 5 \\
4 & $\mathrm{H}$ & 1.0825 & 116.487 & & 1 & 3 & \\
5 & $\mathrm{H}$ & 1.0825 & 121.757 & 0.000 & 2 & 1 & 4 \\
6 & $\mathrm{H}$ & 1.0825 & 121.757 & 180.000 & 2 & 1 & 4 \\
\hline
\end{tabular}

Tabelle A.10: Mit (B3LYP-6-311++G(3df,3pd)) berechnete Gleichgewichtsgeometrie des Ethens $\left(\mathrm{C}_{2} \mathrm{H}_{4}\right)$ im elektronischen Grundzustand. 


\section{A.4.2 Normalmoden}

\begin{tabular}{|c|c|c|ccc|}
\hline $\begin{array}{c}\text { Mode } \\
\text { Nr. }\end{array}$ & Typ & $\begin{array}{c}\text { exp. } \\
{[204]}\end{array}$ & $\mathrm{HF} / \mathrm{B}_{1}$ & $\mathrm{~B} 3 L Y P / \mathrm{B}_{1}$ & B3LYP $/ \mathrm{B}_{2}$ \\
\hline \hline 1 & $\mathrm{CH}_{2}-\operatorname{rock}(\mathrm{s})$ & 826 & 927 & 860 & 836 \\
2 & $\mathrm{CH}_{2}-\operatorname{wag}(\mathrm{s})$ & 943 & 1120 & 1000 & 980 \\
3 & $\mathrm{CH}_{2}-\operatorname{wag}(\mathrm{a})$ & 949 & 1154 & 1003 & 987 \\
4 & $\mathrm{CH}_{2}-\operatorname{twist}$ & 1023 & 1157 & 1073 & 1063 \\
5 & $\mathrm{CH}_{2}-\operatorname{rock}(\mathrm{s})$ & 1236 & 1378 & 1269 & 1241 \\
6 & $\mathrm{CH}_{2}-\operatorname{scissor}(\mathrm{s})$ & 1342 & 1513 & 1415 & 1380 \\
7 & $\mathrm{CH}_{2}-\operatorname{scissor}(\mathrm{a})$ & 1444 & 1632 & 1519 & 1477 \\
8 & $\mathrm{CC}-\operatorname{stretch}$ & 1623 & 1847 & 1714 & 1689 \\
9 & $\mathrm{CH}_{2}-\operatorname{stretch}(\mathrm{s})$ & 2989 & 3314 & 3152 & 3126 \\
10 & $\mathrm{CH}_{2}-\operatorname{stretch}(\mathrm{s})$ & 3026 & 3339 & 3171 & 3140 \\
11 & $\mathrm{CH}_{2}-\operatorname{stretch}(\mathrm{a})$ & 3103 & 3390 & 3227 & 3197 \\
12 & $\mathrm{CH}_{2}-\operatorname{stretch}(\mathrm{a})$ & 3106 & 3420 & 3257 & 3226 \\
\hline
\end{tabular}

Tabelle A.11: Berechnete Normalmoden des Ethens $\left(\mathrm{C}_{2} \mathrm{H}_{4}\right)$ im elektronischen Grundzustand in $\mathrm{cm}^{-1}$.

Basissätze: $\mathrm{B}_{1}=6-31 \mathrm{G}, \mathrm{B}_{2}=6-311++\mathrm{G}(3 \mathrm{df}, 3 \mathrm{dp})$

Die berechneten Frequenzen der Normalmoden des Ethens zeigen, dass die Vergrößerung des Basissatzes von 6-31G auf 6-311++ G(3df,3pd) bei gleicher Methode einen relativ geringen Effekt hat, die Frequenzen reduzieren sich bei Vergrößerung des Basissatzes im Mittel um $30 \mathrm{~cm}^{-1}$ (Tab. A.11). Deutlicher zeigt sich dieser Effekt der Basissatzvergrößerung in den unterschiedlichen Energien, sie wird um $30 \mathrm{kcal} / \mathrm{mol}$ abgesenkt (Tab. A.12).

\section{A.4.3 Energien}

\begin{tabular}{|l|ccc|}
\hline & \multicolumn{3}{|c|}{ Energien in Hartree/Teilchen } \\
& $\mathrm{HF} / \mathrm{B}_{1}$ & $\mathrm{~B} 3 \mathrm{LYP} / \mathrm{B}_{1}$ & $\mathrm{~B} 3 \mathrm{LYP} / \mathrm{B}_{2}$ \\
\hline \hline $\mathrm{E}+\mathrm{ZPE}$ & -77.949343 & -78.520413 & -78.571197 \\
$\mathrm{E}$ & -78.004455 & -78.572037 & -78.622093 \\
\hline
\end{tabular}

Tabelle A.12: Berechnete elektronische Energien (mit/ohne Nullpunktsenergie) des Ethens $\left(\mathrm{C}_{2} \mathrm{H}_{4}\right)$. 


\section{A.5 Hydroxyethenylradikal}

\section{A.5.1 Geometrie}

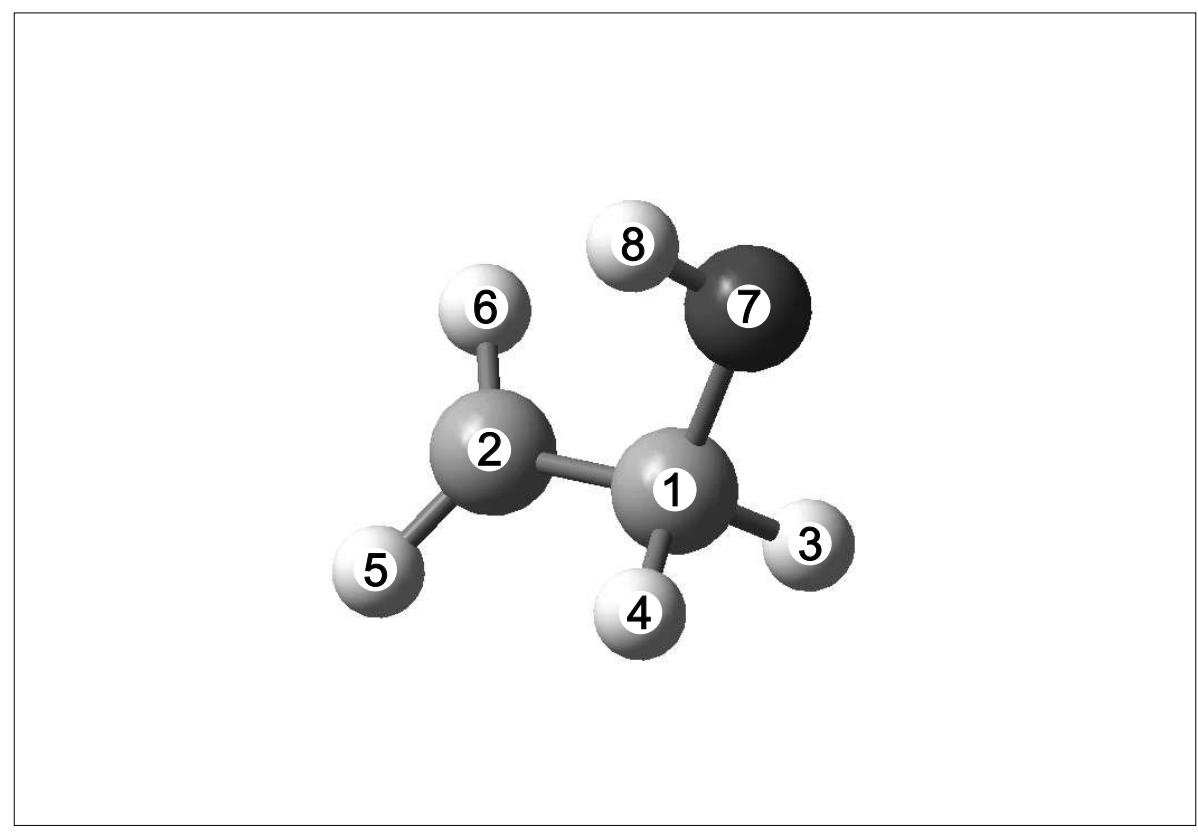

Abbildung A.5: Strukturformel des Hydroxyethenylradikals $\left(\mathrm{C}_{2} \mathrm{H}_{4} \mathrm{OH}\right)$

Das aus der Addition eines OH-Radikals mit Ethen gebildete Hydroxyethenylradikal $\left(\mathrm{C}_{2} \mathrm{H}_{4} \mathrm{OH}\right)$ zeigt eine Lokalisierung des freien Elektrons, analog zum Ethinaddukt, am $\beta$-Kohlenstoffatom. Aufgrund der $\mathrm{sp}^{3}$-Hybridisierung der Kohlenstoffatome ist diese Addukt nicht planar (Abb. A.5, Tab. A.13). In der Arbeit von Liu et al. [50] findet eine Unterscheidung des Adduktes in cis- und trans-Isomere statt, deren Energiedifferenz zu $0.7 \mathrm{kcal} / \mathrm{mol}$ berechnet wurde. Diese Unterscheidung wurde in dieser Arbeit jedoch nicht getroffen.

\section{A.5.2 Normalmoden}

Die berechneten Frequenzen des Adduktes halten einem Vergleich mit einer störungstheoretischen Rechnung [46] stand und weichen nicht-systematisch um ca. $5 \%$ von diesen ab (Tab. A.14). Anzumerken ist, dass die Berechnungen mit dem HF-Modell eine imaginäre Frequenz liefert, die ein Indiz für die Unbrauchbarkeit 


\begin{tabular}{|cc|lcr|rrr|}
\hline $\begin{array}{c}\text { Atom. } \\
\text { Nr. }\end{array}$ & $\begin{array}{c}\text { Chem. } \\
\text { Symb. }\end{array}$ & $\begin{array}{l}\text { Abstand } \\
\text { in }[\AA]\end{array}$ & Winkel & Diederw. & $\mathrm{A}^{(1)}$ & $\mathrm{B}^{(2)}$ & $\mathrm{C}^{(3)}$ \\
\hline \hline 1 & $\mathrm{C}$ & & & & & & \\
2 & $\mathrm{C}$ & 1.4804 & & & 1 & & \\
3 & $\mathrm{H}$ & 1.0967 & 110.514 & & 1 & 2 & \\
4 & $\mathrm{H}$ & 1.0973 & 111.058 & -79.222 & 1 & 2 & 6 \\
5 & $\mathrm{H}$ & 1.0804 & 121.026 & 92.717 & 2 & 1 & 4 \\
6 & $\mathrm{H}$ & 1.0811 & 119.640 & 162.969 & 2 & 1 & 3 \\
7 & $\mathrm{O}$ & 1.4280 & 113.264 & 37.968 & 1 & 2 & 6 \\
8 & $\mathrm{H}$ & 0.9620 & 108.190 & 174.562 & 7 & 1 & 4 \\
\hline
\end{tabular}

Tabelle A.13: Mit (B3LYP-6-311++G(3df,3pd)) berechnete Gleichgewichtsgeometrie Hydroxyethenylradikals $\left(\mathrm{C}_{2} \mathrm{H}_{4} \mathrm{OH}\right)$ im elektronischen Grundzustand.

dieser Ergebnisse ist. Eine spektroskopische Untersuchung des Hyrdoxyethenylradikals wurde von Feltham et al. [205] in einer Argonmatrix bei ca. $12 \mathrm{~K}$ durchgeführt. Dabei konnten mehrere Banden dem Addukt zugeordnet werden, wobei eine starke Überlagerung durch die Edukte vorlag. Es konnten vier Banden zugeordnet werden, die eine zufriedenstellende Übereinstimmung mit den in dieser Arbeit berechneten Werten (Tab. A.14) liefern. Die Signale wurden folgendermassen zugeordnet:

- $872 \mathrm{~cm}^{-1} \rightarrow \mathrm{CH}_{2}$-rocking (berechnet: $827 \mathrm{~cm}^{-1}$ )

- $1043 \mathrm{~cm}^{-1} \rightarrow$ CO-stretch $\left(948 \mathrm{~cm}^{-1}\right)$

- $1173 \mathrm{~cm}^{-1} \rightarrow \mathrm{CH}_{2}$-deformation $\left(1118 \mathrm{~cm}^{-1}\right)$

- $1352 \mathrm{~cm}^{-1} \rightarrow \mathrm{CH}_{2}$-deformation $\left(1357 \mathrm{~cm}^{-1}\right)$

Der Vergleich der Frequenzen der CO-Streckschwingungen der Addukte, die in den SACM-Rechnungen als Reaktionskoordinate dienen, zeigt einen um ca. $150 \mathrm{~cm}^{-1}$ geringeren Wert für das Hydroxyethenylradikal, der auf eine stabilere CO-Bindung für das Hydroxyethinylradikal hindeutet. 


\begin{tabular}{|c|c|ccc|c|}
\hline $\begin{array}{c}\text { Mode } \\
\text { Nr. }\end{array}$ & Typ & HF $/ \mathrm{B}_{1}$ & B3LYP $/ \mathrm{B}_{1}$ & B3LYP $/ \mathrm{B}_{2}$ & Ref. [46] \\
\hline \hline 1 & $\mathrm{CH}_{2}$-twist & -304 & 156 & 177 & 181 \\
2 & OH-torsion & 107 & 353 & 320 & 293 \\
3 & $\mathrm{CH}_{2}$-wag(a) & 412 & 415 & 425 & 411 \\
4 & $\mathrm{CH}_{2}$-wag(s) & 590 & 580 & 545 & 473 \\
5 & $\mathrm{CH}_{2}$-rock(s) & 880 & 828 & 827 & 898 \\
6 & $\mathrm{CO}_{\text {-stretch }}$ & 1008 & 925 & 948 & 1011 \\
7 & $\mathrm{CC}_{-}$stretch & 1128 & 1070 & 1080 & 1113 \\
8 & $\mathrm{CH}_{2}$-rock(a) & 1277 & 1135 & 1118 & 1154 \\
9 & & 1328 & 1201 & 1179 & 1259 \\
10 & & 1410 & 1360 & 1357 & 1301 \\
11 & & 1541 & 1405 & 1396 & 1479 \\
12 & $\mathrm{CH}_{2}$-scissor(a) & 1603 & 1487 & 1453 & 1532 \\
13 & $\mathrm{CH}_{2}$-scissor(s) & 1691 & 1535 & 1482 & 1572 \\
14 & $\mathrm{CH}_{2}$-stretch(s) & 3239 & 3013 & 2972 & 3025 \\
15 & $\mathrm{CH}_{2}$-stretch(a) & 3283 & 3050 & 2994 & 3088 \\
16 & $\mathrm{CH}_{2}$-stretch(s) & 3304 & 3169 & 3142 & 3277 \\
17 & $\mathrm{CH}_{2}$-stretch(a) & 3416 & 3284 & 3252 & 3402 \\
18 & $\mathrm{OH}_{- \text {-stretch }}$ & 4027 & 3614 & 3817 & 3902 \\
\hline
\end{tabular}

Tabelle A.14: Berechnete Normalmoden des Hydroxyethenylradikals $\left(\mathrm{C}_{2} \mathrm{H}_{4} \mathrm{OH}\right)$ in $\mathrm{cm}^{-1}$. Basissätze: $\mathrm{B}_{1}: 6-31 \mathrm{G}, \mathrm{B}_{2}: 6-311++\mathrm{G}(3 \mathrm{df}, 3 \mathrm{dp})$; Ref. [46]: MP2(FULL)/6-31G(d,p)

\section{A.5.3 Energien}

\begin{tabular}{|l|ccc|}
\hline & \multicolumn{3}{|c|}{ Energien in Hartree/Teilchen } \\
& $\mathrm{HF} / \mathrm{B}_{1}$ & $\mathrm{~B} 3 \mathrm{LYP} / \mathrm{B}_{1}$ & $\mathrm{~B} 3 \mathrm{LYP} / \mathrm{B}_{2}$ \\
\hline \hline $\mathrm{E}+\mathrm{ZPE}$ & -153.311275 & -154.253455 & -154.369550 \\
$\mathrm{E}$ & -153.380175 & -154.318569 & -154.434448 \\
\hline
\end{tabular}

Tabelle A.15: Berechnete elektronische Energien (mit/ohne Nullpunktsenergie) des Hydroxyethenylradikals $\left(\mathrm{C}_{2} \mathrm{H}_{4} \mathrm{OH}\right)$. 


\section{A.6 Relative Energien, Reaktionsenthalpien}

Die relativen Energien und Reaktionsenthalpien, die eng miteinander verknüpft sind, werden hier getrennt voneinander betrachtet, um eine bessere Vergleichbarkeit mit anderen Berechnungen und experimentellen Daten zu erreichen. Die angegebenen relativen Energien wurde jeweils aus der Differenz bzw. Summe der berechneten elektronischen Energien (mit Nullpunktsenergie) bestimmt und mit Literaturwerten verglichen.

\begin{tabular}{|c|c|}
\hline $\begin{array}{c}\text { Methode / Basissatz } \\
\text { bzw. Referenz }\end{array}$ & $\mathrm{C}_{2} \mathrm{H}_{4} \mathrm{OH}$ \\
\hline \hline B3LYP/6-311++G(3df,3pd) & -25.5 \\
B3LYP/6-311G(d,p) [50] & -25.3 \\
QCISD(T)/6-311+G(3df,2p) [48] & -26.1 \\
\hline
\end{tabular}

Tabelle A.16: Relative Energien (mit Nullpunktsenergie) des Hydroxyethenylradikals relativ zu den Edukten in $\mathrm{kcal} / \mathrm{mol}$.

Die bestimmte Energie (mit Nullpunktsenergie) des Hydroxyethenylradikals relativ zu den Edukten steht in guter Übereinstimmung mit anderen aktuellen Berechnungen, wobei die QCISD(T)-Rechnung (QCI für „Quadratic Configuration Interaction") von Hippler et al. [48] als die akkurateste angesehen werden kann, da sie die Elektronenkorrelation auf einer höheren Ebene als die $\mathrm{MP}_{4}$-Methode behandelt (A.16). $\mathrm{Zu}$ den relativen Energien des Hydroxyethinylradikals gibt es leider keine Vergleichswerte.

\begin{tabular}{|c|c||c|c|}
\hline $\begin{array}{c}\text { Methode / Basissatz } \\
\text { bzw. Referenz }\end{array}$ & $\begin{array}{c}\mathrm{C}_{2} \mathrm{H}_{2}+\mathrm{OH} \\
\rightarrow \mathrm{C}_{2} \mathrm{H}_{2} \mathrm{OH}\end{array}$ & $\begin{array}{c}\text { Methode / Basissatz } \\
\text { bzw. Referenz }\end{array}$ & $\begin{array}{c}\mathrm{C}_{2} \mathrm{H}_{4}+\mathrm{OH} \\
\rightarrow \mathrm{C}_{2} \mathrm{H}_{4} \mathrm{OH}\end{array}$ \\
\hline \hline B3LYP/B $\mathrm{B}_{2}$ & -33.9 & B3LYP/B & -26.6 \\
exp. [30] & -34.9 & BAC/MP4 [49] & -28.4 \\
exp. [63] & -33.6 & QCISD(T) [48] & -27.0 \\
MP4 [41] & -35.2 & PMP4sdtq [45] & -28.9 \\
[68] (a) & -35.1 & exp. [39] & -30.3 \\
& & MP4 [41] & -27.5 \\
\hline
\end{tabular}

Tabelle A.17: Reaktionsenthalpien $\Delta H^{\circ}(298)$ in $\mathrm{kcal} / \mathrm{mol}$. (a) IUPAC-Empfehlung

Ein weniger theoretischer, und deswegen auch mit experimentellen Daten vergleichbarer Wert ist die Reaktionsenthalpie. In frühen Arbeiten wurden diese Reaktionsenthalpien häufig aus thermodynamischen Überlegungen abgeleitet, d. h. die 
Bildungsenthalpien der beteiligten Moleküle wurden aus Vergleichen mit Molekülen ähnlicher Struktur und bekannter Bildungsenthalpie abgeschätzt und dann kombiniert. Die Ergebnisse dieser Abschätzungen werden hier aber nicht aufgeführt, sondern nur die Werte von anderen theoretischen Studien und experimentelle Werte, die aus der Analyse von temperaturabhängigen Gleichgewichtskonstanten stammen. Die in dieser Arbeit berechnete Reaktionsenthalpie für die Reaktion $\mathrm{C}_{2} \mathrm{H}_{2}+\mathrm{OH} \rightarrow \mathrm{C}_{2} \mathrm{H}_{2} \mathrm{OH}$ stimmt sowohl mit anderen theoretischen Studien als auch mit experimentellen Werten gut überein, der ermittelte Wert für die $\mathrm{C}_{2} \mathrm{H}_{4}+\mathrm{OH} \rightarrow \mathrm{C}_{2} \mathrm{H}_{4} \mathrm{OH}$-Reaktion liegt im Vergleich mit den Literaturwerten vom Betrag her zu niedrig A.17.

Scheinbar liefern die anderen theoretischen Methoden exaktere Werte, obwohl sie den experimentellen Wert ebenfalls nicht erreichen. Der Vergleich der experimentellen Werte beider Reaktionen zeigt eine um ca. 4 kcal/mol größere Reaktionsenthalpie für die Addition des Hydroxylradikals an Ethin als an Ethen, das Hydroxyethinylradikal ist also das stabilere Addukt. Die in dieser Arbeit durchgeführten Rechnungen bestätigen also, dass die Kombination der Methode der Dichtefunktionaltheorie mit genügend großem Basissatz für die Beschreibung von ungesättigten Kohlenwasserstoffen und vor allem für radikalische Spezies sehr gut geeignet ist. Es zeigt sich auch, dass die hier benutzte Methode (B3LYP) in den meisten Fällen mit anderen, zum Teil wesentlich aufwendigeren Methoden (Störungstheorie $\mathrm{MP}_{n}$, Konfigurationswechselwirkung CI) konkurrieren kann, bei der Berechnung von radikalischen Strukturen diesen sogar überlegen ist. 


\section{Anhang B}

\section{SACM-Rechnungen}

Die in dieser Arbeit betrachteten Rekombinationsreaktionen können nicht direkt mit einem Modell für unimolekulare Reaktionen wie dem SAC-Modell simuliert werden. Jedoch kann die unimolekulare Dissoziation des Adduktes berechnet werden $\left(k_{\mathrm{r}}\right)$, über die Gleichgewichtskonstante ergibt sich dann dann die Geschwindigkeitskonstante für die Adduktbildung $\left(k_{\mathrm{f}}\right)$ :

$$
\begin{aligned}
\mathrm{C}_{2} \mathrm{H}_{2 n}+\mathrm{OH} & \leftrightharpoons \mathrm{C}_{2} \mathrm{H}_{2 n} \mathrm{OH} \quad n=1 ; 2 \\
k_{\mathrm{f}} & =K_{\mathrm{eq}} k_{\mathrm{r}}=\frac{Q_{\mathrm{C}_{2} \mathrm{H}_{2 n} \mathrm{OH}}}{Q_{\mathrm{OH}} Q_{\mathrm{C}_{2} \mathrm{H}_{2 n}}} \cdot k_{\mathrm{r}}
\end{aligned}
$$

Die Zustandssummen $Q_{\mathrm{i}}$ können aus den berechneten Molekülparametern bestimmt werden. Die auf der SACM-Theorie (vgl. Abschnitt 2.1.3) basierende Abschätzung der Geschwindigkeitskonstanten für die Dissoziation der Addukte wurde mit einem FORTRAN-Programm von Borrel und Brower, welches später von Troe modifiziert wurde $[112,122,206,207,208]$ durchgeführt. Dazu wurden die nötigen Molekülparameter sowie die Korrelation der Edukt- bzw. Produktoszillatoren mit dem Programm DATAPREP in einer Eingabedatei gespeichert, die eigentliche Berechnung der Geschwindigkeitskonstanten erfolgte mit dem Programm FIMAIN.

\section{B.1 Korrelation der Edukt- und Produktoszillatoren nach dem SAC-Modell}

Die Korrelationen der Oszillatoren von Edukten und Produkten der beiden betrachteten Reaktionen sind in Tab. B.1 und B.2 aufgeführt. In beiden Fällen wurde die CO-Streckschwingung $\left(1098 \mathrm{~cm}^{-1}\right.$ für $n=1$ bzw. $948 \mathrm{~cm}^{-1}$ für $\left.n=2\right)$ als Reaktionskoordinate gewählt, da sie den Fortgang der Reaktion, also die Entfernung (bzw. 
Annäherung) des Hydroxylradikals mit dem Sauerstoffatom an ein Kohlenstoffatom, am zutreffendsten beschreibt. Ebenfalls für beide Reaktionen wird die $\mathrm{OH}-$ Torsionsschwingung $\left(467 \mathrm{~cm}^{-1}\right.$ für $n=1 \mathrm{bzw} .320 \mathrm{~cm}^{-1}$ für $\left.n=2\right)$ des Adduktes in

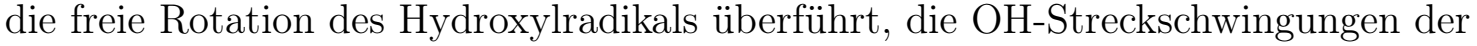
Addukte bleibt im Hydroxylradikal bestehen und verringert sich bei der Dissoziation des Adduktes um $70 \mathrm{bzw} .100 \mathrm{~cm}^{-1}$. Als sogenannte verschwindende Oszillatoren werden diejenigen Schwingungen bezeichnet, die bei der Dissoziation des Adduktes wegfallen; in beiden Fällen sind dies logischerweise die mit dem Sauerstoffatom assoziierten Schwingungen (Nr. 2 und 8 für $n=1$, Nr. 9, 10 und 11 für $n=2$ ). Alle hier aufgeführten Frequenzen wurden mit B3LYP $/ 6-311++\mathrm{G}(3 \mathrm{df}, 3 \mathrm{pd})$ berechnet.

\section{B.1.1 Ethin $+\mathrm{OH}$}

\begin{tabular}{|c|c|c|c|c|}
\hline $\begin{array}{c}\text { Mode } \\
\text { Nr. }\end{array}$ & Hydroxyethinylradikal & & $\begin{array}{c}\text { Mode } \\
\text { Nr. }\end{array}$ & Ethin $/ \mathrm{OH}$ \\
\hline \hline 1 & 467 & $\rightarrow$ & OH-Rotation & $\mathrm{B}=18.91 \mathrm{~cm}^{-1}$ \\
2 & 474 & $\rightarrow$ & & 657 \\
3 & 569 & $\rightarrow$ & 1 & 765 \\
4 & 769 & $\rightarrow$ & 3 & 657 \\
5 & 843 & $\rightarrow$ & 2 & 765 \\
6 & 1098 & $\rightarrow$ & $\mathrm{q}$ & \\
7 & 1244 & $\rightarrow$ & 4 & 2066 \\
8 & 1355 & $\rightarrow$ & & 3407 \\
9 & 1667 & $\rightarrow$ & 5 & 3509 \\
10 & 3107 & $\rightarrow$ & 6 & 3714 \\
11 & 3307 & $\rightarrow$ & 7 & Reaktionskoordinate \\
12 & 3786 & $\rightarrow$ & OH & \\
\hline
\end{tabular}

Tabelle B.1: Berechnete Normalmoden des Hydroxyethinylradikals $\left(\mathrm{C}_{2} \mathrm{H}_{2} \mathrm{OH}\right)$, Ethins und des Hydroxylradikals in $\mathrm{cm}^{-1}$. 


\section{B.1.2 Ethen $+\mathrm{OH}$}

\begin{tabular}{|c|c|c|c|c|}
\hline $\begin{array}{c}\text { Mode } \\
\text { Nr. }\end{array}$ & Hydroxyethenylradikal & & $\begin{array}{c}\text { Mode } \\
\text { Nr. }\end{array}$ & Ethen $/ \mathrm{OH}$ \\
\hline \hline 1 & 177 & $\rightarrow$ & 4 & 1062 \\
2 & 320 & $\rightarrow$ & OH-Rotation & B = 18.91 cm $\mathrm{cm}^{-1}$ \\
3 & 425 & $\rightarrow$ & 3 & 987 \\
4 & 545 & $\rightarrow$ & 2 & 980 \\
5 & 827 & $\rightarrow$ & 1 & 836 \\
6 & 948 & $\rightarrow$ & $\mathrm{q}$ & Reaktionskoordinate \\
7 & 1080 & $\rightarrow$ & 8 & 1689 \\
8 & 1118 & $\rightarrow$ & 5 & 1241 \\
9 & 1179 & $\rightarrow$ & & \\
10 & 1357 & $\rightarrow$ & & 1477 \\
11 & 1396 & $\rightarrow$ & 7 & 1380 \\
12 & 1453 & $\rightarrow$ & 6 & 3140 \\
13 & 1482 & $\rightarrow$ & 10 & 3197 \\
14 & 2972 & $\rightarrow$ & 11 & 3126 \\
15 & 2994 & $\rightarrow$ & 9 & 3226 \\
16 & 3143 & $\rightarrow$ & 12 & 3714 \\
17 & 3252 & $\rightarrow$ & OH & \\
18 & 3817 & & & \\
\hline
\end{tabular}

Tabelle B.2: Berechnete Normalmoden des Hydroxyethenylradikals $\left(\mathrm{C}_{2} \mathrm{H}_{2} \mathrm{OH}\right)$, Ethens und des Hydroxylradikals in $\mathrm{cm}^{-1}$. 


\section{B.2 Parameter für die SACM-Rechnungen}

Die weiteren für die SACM-Rechnungen nötigen Parameter sind in Tab. B.3 und B.4 angegeben. Dabei ist zu erkennen, dass sowohl die Dissoziationsenergie, der Morseparameter und damit auch die Kraftkonstante der zu brechenden Bindung für das Hydroxyethinylradikal größer sind als die des Hydroxyethenylradikal.Im Vergleich der beiden Morsepotentiale fällt auf, dass der Anstieg zu größeren Abständen, der durch $\beta$ bestimmt ist, für das Hydroxyethenylradikal wesentlich flacher ist. Es ist jedoch zu beachten, dass das Morsepotential nur eine Annäherung des tatsächlichen Verlaufs der elektronischen Energie mit dem Fragmentabstand ist, gerade der Anstieg bei größeren Abständen wird durch ein Morsepotential unzureichend beschrieben. Allerdings sollte der Trend im Anstieg der beiden realen Potentiale qualitativ richtig beschrieben sein. Die Dissoziationsenergie $\mathrm{D}_{0}$ wurde als Summe der elektronischen Energien der beteiligten Moleküle bestimmt, der Morseparameter $\beta$ wurde mit Hilfe von $\mathrm{D}_{0}$ und der Kraftkonstanten $\mathrm{F}_{\mathrm{q}}$ des Oszillators, der die Reaktionskoordinate $q$ repräsentiert, nach Gl. 2.23 berechnet. Die Parameter $a_{1}$ und $a_{1}$ wurden aus $\mathrm{D}_{0}$ und dem Gleichgewichtsabstand auf der Reaktionskoordinate $q_{e}$ bestimmt:

$$
a_{1}=\frac{2}{\beta q_{e}} \quad a_{2}=\frac{1}{\left(\beta q_{e}\right)^{2}}
$$

Der Anisotropie- oder Paulingparameter $\alpha$ wurde durch den Vergleich mit den experimentellen Daten aus Abschnitt 4.2 angepasst. Alle Parameter und die daraus abgeleiteten Größen sind Ergebnisse der quantenchemischen Rechnungen mit der folgenden Methode bzw. dem Basissatz B3LYP/6-311 ++G(3df,3pd).

\begin{tabular}{|c|c|c|}
\hline & $\mathrm{C}_{2} \mathrm{H}_{2} \mathrm{OH}$ & $\mathrm{C}_{2} \mathrm{H}_{4} \mathrm{OH}$ \\
\hline \hline $\mathrm{D}_{0}\left[\mathrm{~cm}^{-1}\right]$ & 11323 & 8905 \\
$\beta\left[\AA^{-1}\right]$ & 2.49 & 2.06 \\
$a_{1}$ & 0.589 & 0.682 \\
$a_{2}$ & 0.087 & 0.116 \\
$\mathrm{~F}_{\mathrm{q}}[\mathrm{N} / \mathrm{m}]$ & 318.24 & 149.5 \\
$q_{e}[\AA]$ & 1.36 & 1.36 \\
\hline
\end{tabular}

Tabelle B.3: Berechnete Parameter der Addukte. 


\begin{tabular}{|c|c|cc|cc|}
\hline & $\mathrm{OH}$ & $\mathrm{C}_{2} \mathrm{H}_{2}$ & $\mathrm{C}_{2} \mathrm{H}_{2} \mathrm{OH}$ & $\mathrm{C}_{2} \mathrm{H}_{4}$ & $\mathrm{C}_{2} \mathrm{H}_{4} \mathrm{OH}$ \\
\hline \hline $\mathrm{A}$ & & & 2.6423 & 4.9327 & 1.2897 \\
$\mathrm{~B}$ & 18.91 & 1.17 & 0.3643 & 1.0119 & 0.3288 \\
$\mathrm{C}$ & & & 0.3201 & 0.8397 & 0.2854 \\
\hline
\end{tabular}

Tabelle B.4: Berechnete Rotationskonstanten der beteiligten Moleküle in $\mathrm{cm}^{-1}$.

\section{B.2.1 Ethin $+\mathrm{OH}$}

Die in Abb. B.1 gezeigte doppelt logarithmische Auftragung der nach dem SACModell abgschätzten Rekombinationsgeschwindigkeitskonstanten gegen die Temperatur zeigt die Abhängigkeit von $\alpha / \beta$. Dabei wurde der Wert des Morseparameters $\beta$ konstant gehalten und der Anisotropieparameter $\alpha$ variiert, eine Variation von $\beta$ bei konstantem $\alpha$ hat jedoch denselben Effekt, so dass sich die SACM-Rechnungen am besten durch das Verhältnis von $\alpha$ und $\beta$ vergleichen lassen. Bei hohen Werten von $\alpha / \beta$ ist ein monotoner Anstieg der Geschwindigkeitskonstanten mit sinkender Temperatur zu beobachten, dies ändert sich ab einem Verhältnis von ca. 0.4, ab diesem Wert sinken die Geschwindigkeitskonstanten mit sinkender Temperatur. Das dadurch entstehende Maximum verschiebt sich mit kleiner werdendem $\alpha / \beta$ zu höheren Temperaturen. Der Vergleich der in dieser Arbeit durchgeführten Abschätzung der Geschwindigkeit der Additionsreaktion mit den experimentellen Daten findet sich in Abschnitt 5.

\section{B.2.2 Ethen $+\mathrm{OH}$}

Die SACM-Modellierung zur Reaktion des Ethen mit OH-Radikalen zeigen einen ähnlichen Trend (vgl. Abb. B.2). Bei kleiner werdendem $\alpha / \beta$ bildet sich ein Maximum in der Temperaturabhängigkeit der Geschwindigkeitskonstanten aus, jedoch erst bei kleineren Werten (ab ca. 0.3) als bei der entsprechenden Reaktion des Ethins. Die Variation von $\alpha / \beta$ wurde analog wie im vorhergehenden Abschnitt bei einem konstantem Wert für $\beta$ durchgeführt. 


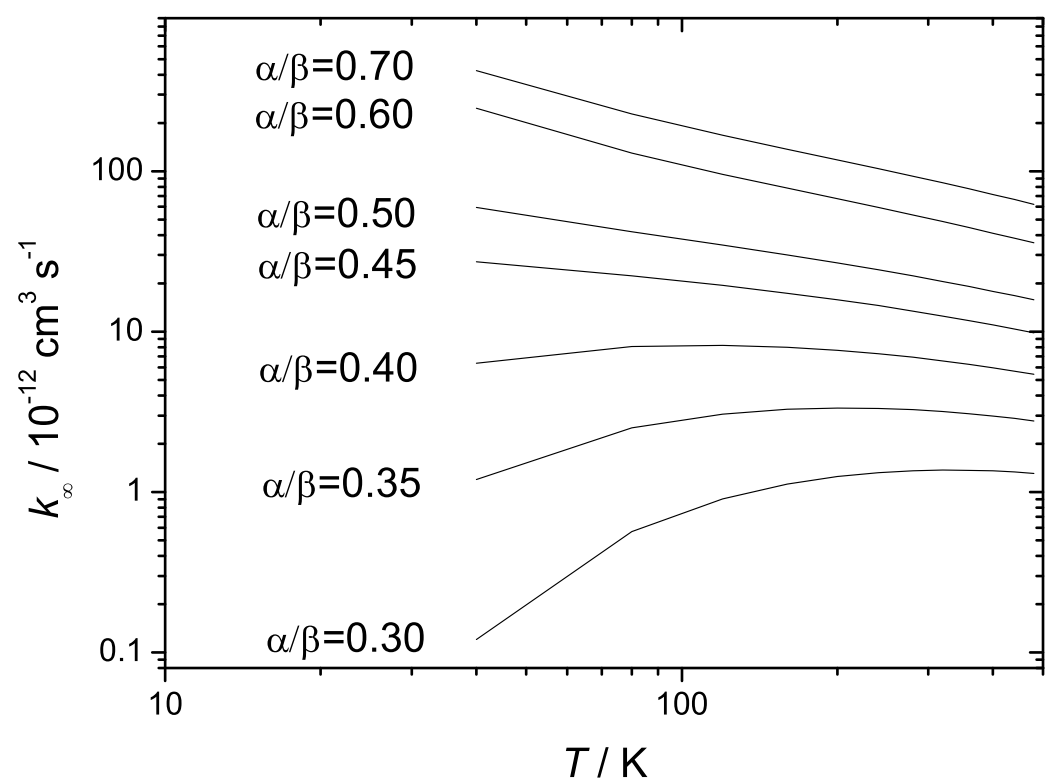

Abbildung B.1: Parameterstudie der Hochdruckgeschwindigkeitskonstanten für die Reaktion $\mathrm{C}_{2} \mathrm{H}_{2}+\mathrm{OH} ; \alpha$ : Anisotropieparameter, $\beta$ : Morseparameter

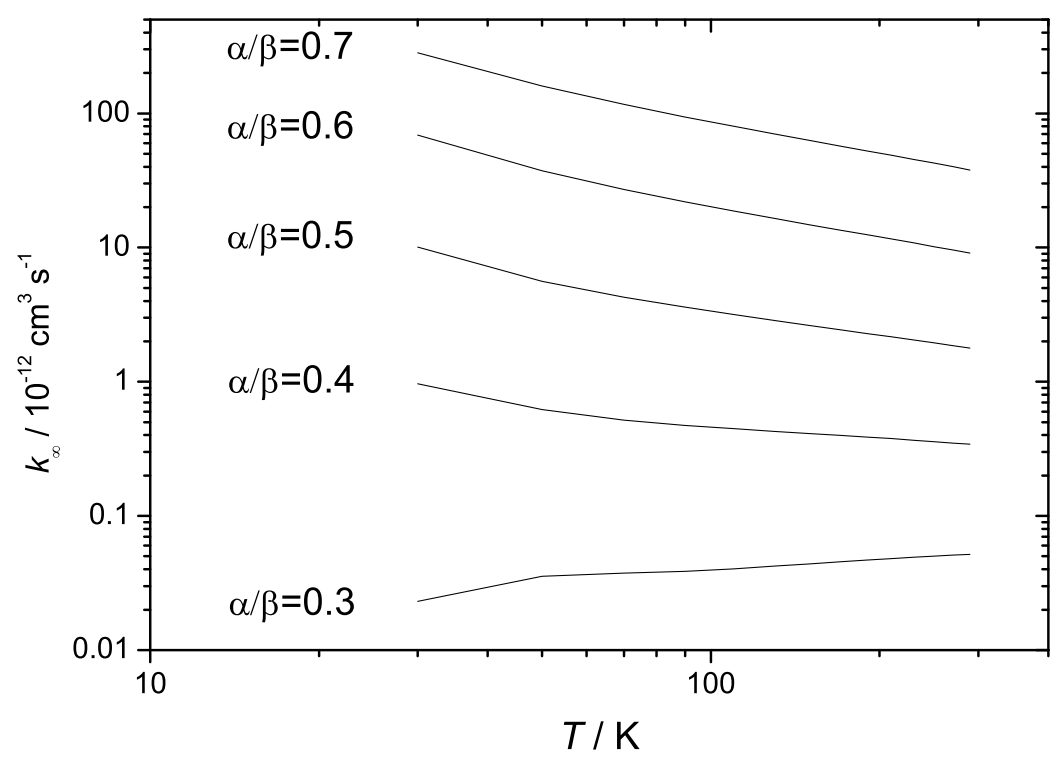

Abbildung B.2: Parameterstudie der Hochdruckgeschwindigkeitskonstanten für die Reaktion $\mathrm{C}_{2} \mathrm{H}_{4}+\mathrm{OH} ; \alpha$ : Anisotropieparameter, $\beta$ : Morseparameter 


\section{Abbildungsverzeichnis}

1.1 Reaktion 1.8 in Anwesenheit von $\mathrm{O}_{2} \ldots \ldots \ldots$. . . . . . . 6

1.2 Isomerisierung zum Vinoxyradikal . . . . . . . . . . . . . . . . . 7

1.3 Isomere OH-Propen-Addukte . . . . . . . . . . . . . . . . 7

1.4 Isomere OH-Isopren-Addukte . . . . . . . . . . . . . . 8

1.5 Hauptprodukte der Oxidation von Isopren in der Atmosphäre . . . 9

1.6 Schematischer Energieverlauf einer Reaktion mit Barriere . . . . . . 11

1.7 Schematischer Energieverlauf einer Reaktion ohne Barriere . . . . . 12

2.1 Fall-Off-Kurve . . . . . . . . . . . . . . . . . . . . . 19

2.2 Mikroskopisches Bild einer unimolekularer Reaktion . . . . . . . . . 20

2.3 Adiabatische Potentialkurven ................ 24

2.4 Potentialenergien und ihr Zusammenhang . . . . . . . . . . 26

3.1 Expansionsarten . . . . . . . . . . . . . . . . . 40

3.2 Prinzip der Lavaldüsenexpansion . . . . . . . . . . . . . . . . 41

3.3 Schema einer Lavaldüse . . . . . . . . . . . . . . . . . . . . . . . . 44

3.4 Isentroper Kern und Grenzschicht . . . . . . . . . . . . . . . . . 45

3.5 Regionen der Lavaldüse . . . . . . . . . . . . . . . . . . . . 46

3.6 Region II der Lavaldüse . . . . . . . . . . . . . . . . . . . . . . . . 47



3.8 Aufbau der gepulsten CRESU-Apparatur . . . . . . . . . . . . 51

3.9 Zeitliche Steuerung des Experimentes . . . . . . . . . . . . 56

3.10 Spektrum des OH-Radikals . . . . . . . . . . . . . . 59

$3.11 S_{21}(K)$-Zweig . . . . . . . . . . . . . . . . 60

3.12 Boltzmann-Auftragung . . . . . . . . . . . . . . . 60

3.13 Temperaturprofil $(\mathrm{LIF}) \ldots \ldots \ldots$. . . . . . . . . . . . . . . . . . . . . . . . . 61

3.14 Temperaturprofil (Druckmessung) . . . . . . . . . . . 61

3.15 Dichteprofil ... . . . . . . . . . . . . . . . 63

3.16 3D-Abbild der Expansion . . . . . . . . . . . . . 63

4.1 Rotationsrelaxation des Hydroxylradikals . . . . . . . . . . . . . 66 
4.2 Schwingungsrelaxation des OH-Radikals (in $\mathrm{C}_{2} \mathrm{H}_{4} / \mathrm{N}_{2}$ ); $T=98 \mathrm{~K}$.

4.3 Kinetiken pseudo-erster Ordnung bei verschiedener Reaktandenkonzentration $\left(\mathrm{OH}\left(v^{\prime \prime}=0\right)+\mathrm{C}_{2} \mathrm{H}_{2}\right) \ldots \ldots \ldots$

4.4 Kinetiken pseudo-erster Ordnung bei verschiedener Reaktandenkon-

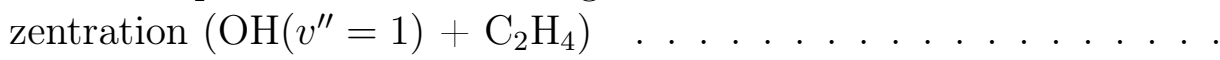

4.5 Geschwindigkeitskonstanten pseudo-erster Ordnung in Abhängigkeit der Reaktandenkonzentration $\left(\mathrm{OH}\left(v^{\prime \prime}=0\right)+\mathrm{C}_{2} \mathrm{H}_{2}\right) \ldots . . . .70$

4.6 Geschwindigkeitskonstanten pseudo-erster Ordnung in Abhängigkeit der Reaktandenkonzentration $\left(\mathrm{OH}\left(v^{\prime \prime}=1\right)+\mathrm{C}_{2} \mathrm{H}_{4}\right)$. . . . . . . . 70

4.7 Geschwindigkeitskonstanten 2. Ordnung für $\left(\mathrm{OH}+\mathrm{C}_{2} \mathrm{H}_{2}\right)$. . . . . 73

4.8 Geschwindigkeitskonstanten 2. Ordnung für $\left(\mathrm{OH}+\mathrm{C}_{2} \mathrm{H}_{4}\right)$. . . . . 75

4.9 Auftragung der in dieser Arbeit bestimmten Geschwindigkeitskonstanten für $\mathrm{OH}+$ Propen . . . . . . . . . . . . . . . . . . . . 77

4.10 Auftragung der in dieser Arbeit bestimmten Geschwindigkeitskonstanten für $\mathrm{OH}+$ Isopren . . . . . . . . . . . . . . . 78

5.1 Geschwindigkeitskonstanten $k_{\infty}$ für die Reaktion des $\mathrm{OH}$ mit Ethin 80

5.2 Geschwindigkeitskonstanten $k_{\infty}$ für die Reaktion des $\mathrm{OH}$ mit Ethen 84

5.3 Isomere OH-Propen-Addukte . . . . . . . . . . . . 86

5.4 Auftragung der bisherigen Geschwindigkeitskonstanten für die Reaktion des $\mathrm{OH}$ mit Propen . . . . . . . . . . . . . . . . 87

5.5 Auftragung der bisherigen Geschwindigkeitskonstanten für die Reaktion des $\mathrm{OH}$ mit Isopren . . . . . . . . . . . . . . . . . . . 89

5.6 Isomere OH-Isopren-Addukte . . . . . . . . . . . . . . . . . . 90

5.7 Die Reaktionen des Hydroxylradikals mit verschiedenen Alkenen bei tiefen Temperaturen . . . . . . . . . . . . . . . . . . 91

5.9 Schematischer Verlauf der potentiellen Energie der Rekombination von Hydroxylradikalen mit ungesättigten Kohlenwasserstoffen . . . 95

A.1 Strukturformel des Hydroxylradikals . . . . . . . . . . . . . . 106

A.2 Strukturformel des Ethins $\left(\mathrm{C}_{2} \mathrm{H}_{2}\right) \ldots \ldots \ldots$. . . . . . . . . 108

A.3 Strukturformel des Hydroxyethinylradikals $\left(\mathrm{C}_{2} \mathrm{H}_{2} \mathrm{OH}\right)$. . . . . . . 110

A.4 Strukturformel des Ethens $\left(\mathrm{C}_{2} \mathrm{H}_{4}\right) \ldots \ldots$. . . . . . . . . . 112

A.5 Strukturformel des Hydroxyethenylradikals $\left(\mathrm{C}_{2} \mathrm{H}_{4} \mathrm{OH}\right)$. . . . . . . 114

B.1 Parameterstudie der Geschwindigkeitskonstanten $k_{\infty}$ für $\mathrm{C}_{2} \mathrm{H}_{2}+\mathrm{OH} 124$

B.2 Parameterstudie der Geschwindigkeitskonstanten $k_{\infty}$ für $\mathrm{C}_{2} \mathrm{H}_{4}+\mathrm{OH} 124$ 


\section{Literaturverzeichnis}

[1] J. Rudolph, in G. Helas, J. Slanina und R. Steinbrecher (Hg.), "Biogenic Volatile Organic Carbon Compounds in the Atmosphere", Academic Publishing, Amsterdam, 1997 53-65. 3

[2] D. W. Arlander, D. R. Cronn, J. C. Farmer, F. A. Menzia und H. H. Westberg, J. Geophys. Res., 1990, 95, 16 391-16 403. 3

[3] J. Rudolph und F. J. Johnen, J. Geophys. Res., 1990, 95, 20 583-20 591. 3

[4] B. Bonsang, D. Martin, G. Lambert, M. Kanakidou, L. C. LeRoulley und G. Sennequier, J. Geophys. Res., 1991, 96, 7313-7324. 3

[5] C. Plass-Dülmer, A. Khedim, R. Koppmann, F. J. Johnen, J. Rudolph und H. Kuosa, Global Biogeochem. Cycl., 1993, 7, 211-228. 3

[6] I. Diaz-Costa, R. Alvarez-Idaboy und A. Vivier-Bunge, Int. J. Chem. Kinet., 1999, 31 (1), 29. 3, 7, 8, 85, 93

[7] T. D. Sharkey und E. L. Singsaas, Nature, 1995, 374, 769. 3

[8] W. S. McGivern, I. Suh, A. D. Clinkenbeard, R. Zhang und S. W. North, J. Phys. Chem. A, 2000, 104, 6609. 3, 8, 88, 89

[9] R. Atkinson, Chem. Rev., 1985, 85 (1), 69-201. 3

[10] E. D. J. Morris, D. H. Stedman und H. Niki, J. Am. Chem. Soc., 1971, 93 (15), 3570-3572. 4

[11] K. Hoyermann und R. Sievert, Ber. Bunsenges. Phys. Chem., 1983, 87, 10271031. 4

[12] N. R. Greiner, J. Chem. Phys., 1970, 53 (3), 1284-1285. 4, 83

[13] F. Stuhl, Ber. Bunsenges., 1973, 77 (9), 674-677. 4 
[14] I. W. M. Smith und R. Zellner, J. Chem. Soc. Farad.Trans. 2, 1973, 69 (11), 1617-1627. 4, 5

[15] J. N. Bradley, W. Hack, K. Hoyermann und H. G. Wagner, J. Chem. Soc. Faraday Trans. 1, 1973, 69 (11), 1889-1898. 4

[16] D. D. Davis, S. Fischer, R. Schiff, R. T. Watson und W. Bollinger, J. Chem. Phys., 1975, 63 (5), 1707-1712. 4, 5

[17] H. B. Palmer, J. Chem . Phys., 1976, 64 (6), 2699-2700. 4

[18] A. C. Lloyd, K. R. Darnall, A. M. Winer und J. Pitts, J. N., J. Phys. Chem., 1976, 80 (8), 789-794. 4

[19] J. F. Meagher und J. Heicklen, J. Phys. Chem., 1976, 80 (15), 1645-1652. 4

[20] C. J. Howard, J. Chem. Phys., 1976, 65 (11), 4771-4777. 4

[21] R. Atkinson, R. A. Perry und J. Pitts, J. N., J. Chem. Phys., 1977, 66 (3), 1197-1201. 4, 83

[22] R. Overend und G. Paraskevopoulos, J. Chem. Phys., 1977, 67 (2), 674-679. 4

[23] R. A. Cox, R. G. Derwent und M. R. Williams, Environ. Sci. Technol., 1980, $14(1), 57-61.4,8$

[24] G. K. Farquharson und R. H. Smith, Austr. J. Chem., 1980, 33 (7), 14251435. 4

[25] R. Atkinson, S. M. Aschmann, A. M. Winer und J. Pitts, J. N., Int. J. Chem. Kinet., 1982, 14 (5), 507-516. 4, 8

[26] F. P. Tully, Chem. Phys. Lett., 1983, 96 (2), 148-153. 4

[27] R. Zellner und K. Lorenz, J. Phys. Chem., 1984, 88 (5), 984-990. 4, 82, 83, 84

[28] T. Klein, I. Barnes, E. H. Fink und F. Zabel, J. Phys. Chem., 1984, 88 (21), 5020-5025. 4, 8, 76, 82, 83, 84, 85, 86, 87

[29] R. Atkinson und S. M. Aschmann, Int. J. Chem. Kinet., 1984, 16 (3), 259268. $4,5,82,85,86,87$

[30] D. Fulle, H. F. Hamann, H. Hippler und C. P. Jänsch, Ber. Bunsenges. Phys. Chem., 1997, 101 (10), 1433-1442. 4, 5, 72, 73, 75, 76, 79, 80, 81, 82, 83, 84, 117 
[31] B. Chuong und P. S. Stevens, J. Phys. Chem. A, 2000, 104 (22), 5230-5237. 4, 8, 77, 88, 89

[32] J. N. Bradley, W. D. Capey, R. W. Fair und D. K. Pritchard, Int. J. Chem. Kinet., 1976, 8 (4), 549-561. 4

[33] A. D. Liu, W. A. Mulac und C. D. Jonah, Int. J. Chem. Kinet., 1987, 19 (1), $25-34.4$

[34] A. Liu, W. A. Mulac und C. D. Jonah, J. Phys. Chem., 1988, 92 (13), 38283833. 4

[35] F. P. Tully, Chem. Phys. Lett., 1988, 143 (5), 510-514. 4

[36] C. H. Kuo und Y. P. Lee, J. Phys. Chem., 1991, 95 (3), 1253-1257. 4

[37] J. F. Bott und N. Cohen, Int. J. Chem. Kinet., 1991, 23 (12), 1075-1094. 4, 5

[38] A. Bhargava und P. R. Westmoreland, Chem. Phys. Proc. Combust., 1997, 201-204. 4

[39] E. W. G. Diau und Y. P. Lee, J. Chem. Phys., 1992, 96 (1), 377-386. 4, 82, $83,84,117$

[40] H. Guesten, W. G. Filby und S. Schoof, Atmos. Env., 1981, 15 (9), 17631765. 4

[41] C. Sosa und H. B. Schlegel, J. Am. Chem. Soc., 1987, 109 (14), 4193-4198. $4,5,81,84,93,117$

[42] C. Sosa und H. B. Schlegel, J. Am. Chem. Soc., 1987, 109 (23), 7007-7015. 4

[43] B. V. Cheney, Theochem, 1996, 364 (2-3), 219-237. 4

[44] T. Yamada, J. W. Bozelli und T. Lay, Chemical and Physical Processes in Combustion, 1996, 523-526. 4

[45] J. Villa, A. Gonzales-Lafont, J. M. Lluch, J. C. Corchado und J. Espinosa-



[46] T. Yamada, J. W. Bozelli und T. Lay, J. Phys. Chem. A, 1999, 103 (38), 7646-7655. 4, 114, 116

[47] J. R. Alvarez-Idaboy, N. Mora-Diez und A. Vivier-Bunge, J. Am. Chem. Soc., 2000, 122 (15), 3715-3720. 4, 82, 83, 84, 85, 87 
[48] H. Hippler und B. Viskolcz, Phys. Chem. Chem. Phys., 2000, 2 (16), 35913596. 4, 84, 117

[49] M. C. Piqueras, R. Crespo, I. Nebot-Gil und F. Tomas, Theochem, 2001, 537, 199-212. 4, 84, 93, 117

[50] G. X. Liu, Y. H. Ding, Z. S. Li, Q. Fu, X. R. Huang, C. C. Sun und A. C. Tang, Phys. Chem. Chem. Phys., 2002, 4 (6), 1021-1027. 4, 84, 114, 117

[51] M. Gehring, K. Hoyermann, H. G. Wagner und J. Wolfrum, Z. f. Naturf. A: Astrophysik, Physik und Physikalische Chemie, 1970, 25 (5), 675-676. 5

[52] J. E. Breen und G. P. Glass, Int. J. Chem. Kinet., 1971, 3 (2), 145-153. 5

[53] J. R. Kanofsky, D. Lucas, F. Pruss und D. Gutman, J. Phys. Chem., 1974, 78 (4), 311-316. 5

[54] V. A. Pastrana und J. Carr, R. W., Int. J. Chem. Kinet., 1974, 6 (4), 587595. 5

[55] R. A. Perry, R. Atkinson und J. N. J. Pitts, J. Chem. Phys., 1977, 67 (12), $5577-5584.5$

[56] J. V. Michael, D. F. Nava, R. P. Borkowski, W. A. Payne und L. J. Stief, J. Chem. Phys., 1980, 73 (12), 6108-6116. 5, 74

[57] R. A. Perry und D. Williamson, Chem. Phys. Lett., 1982, 93 (4), 331-334. 5

[58] W. Hack, K. Hoyermann, R. Sievert und H. G. Wagner, Ox. Comm., 1983, 5 (1-2), 104-114. 5

[59] A. Wahner und C. Zetzsch, Ber. Bunsenges. Phys. Chem., 1985, 89 (3), 323325. $5,79,80$

[60] V. Schmidt, G. Y. Zhu, K. H. Becker und E. H. Fink, Ber. Bunsenges. Phys. Chem., 1985, 89 (3), 321-322. 5, 6, 79, 80

[61] S. Hatakeyama, N. Washida und H. Akimoto, J. Phys. Chem., 1986, 90 (1), 173-178. 5, 6, 82

[62] R. R. Arnts, R. L. Seila und J. J. Bufalini, JAPCA, 1989, 39 (4), 453-460. 5

[63] L. H. Lai, Y. C. Hsu und Y. P. Lee, J. Chem. Phys., 1992, 97 (5), 3092-3099. $5,7,117$

[64] C. Y. Lin und M. C. Lin, Chem. Phys. Proc. Combust., 1984, 86, 1-4. 5, 7 
[65] G. P. Smith, P. W. Fairchild und D. R. Crosley, J. Chem. Phys., 1984, 81 (6), 2667-2677. 5, 7

[66] A. Liu, W. A. Mulac und C. D. Jonah, J. Phys. Chem., 1988, 92 (21), 59425945. 5, 79, 80, 81, 82, 83, 84

[67] E. W. Kaiser, J. Phys. Chem., 1990, 94 (11), 4493-4499. 5

[68] R. Atkinson, D. L. Baulch, R. A. Cox, R. F. Hampson, Jr., J. A. Kerr, M. J. Rossi und J. Troe, J. Phys. Chem. Ref. Data, 1999, 28 (191). 6, 72, 75, 80, 117

[69] M. Siese und C. Zetsch, Z. Phys. Chem., 1995, 188 (1/2), 75-89. 6

[70] B. Bohn, M. Siese und C. Zetsch, J. Chem. Soc. Faraday Trans., 1996, 92 (9), 1459-1466. 6

[71] B. Bohn und C. Zetsch, J. Chem. Soc. Faraday Trans., 1998, 94, 1203-1210. 6

[72] J. A. Miller und C. F. Melius, in "Symp. (Int.) Combust.", Bd. 22, 1988 10311039. 7, 79, 80, 81

[73] R. Atkinson und J. N. Pitts, J. Chem. Phys., 1975, 63, 3591. 8

[74] A. R. Ravishankara, S. Wagner, S. Fischer, G. Smith, R. Schiff, R. T. Watson, G. Tesi und D. D. Davis, Int. J. Chem. Kinet., 1978, 10, 783. 8

[75] R. Atkinson und S. M. Aschmann, Int. J. Chem. Kinet., 1984, 16 (10), 11751186. 8

[76] R. Atkinson, J. Phys. Chem. Data Monograph No. 1, 1989, 1-246. 8

[77] O. J. Nielsen, O. Jorgensen, M. Donlon, H. W. Sidebottom, D. J. OFarell und J. Treacy, Chem. Phys. Lett., 1990, 168, 319. 8

[78] A. B. Vakhtin, D. E. Heard, I. W. M. Smith und S. R. Leone, Chem. Phys. Lett., 2001, 348, 21-26. 8, 42, 86, 87, 98

[79] R. H. Smith, J. Phys. Chem., 1983, 87 (9), 1596. 8, 85

[80] P. S. Stevens, E. Seymour und L. Zhuangjie, J. Phys. Chem. A, 2000, 104, 5989. 8, 9

[81] S. E. Paulson, R. C. Flagan und J. H. Seinfeld, Int. J. Chem. Kinet., 1992, 24, 79. 8

[82] T. J. Otha, J. Phys. Chem., 1983, 87, 1209-1213. 8 
[83] E. O. Edney, T. E. Kleindienst und E. W. Corse, Int. J. Chem. Kinet., 1986, $18,1355-3171.8$

[84] R. Zhang, I. Suh, W. Lei, A. D. Clinkenbeard und S. W. North, J. Geophys. Res., 2000, 105 (D20), 24627-24636. 8

[85] P. Campuzano-Jost, M. B. Williams, L. D'Ottone und A. Hynes, Geophys. Res. Lett., 2000, ${ }^{77}$ (5), 693-696. 8, 88, 89

[86] T. E. Kleindienst, G. W. Harris und J. N. Pitts, Environ. Sci. Technol., 1982, 16, 844-846. 9, 88, 89

[87] K. J. Gill und R. A. Hites, J. Phys. Chem. A, 2002, 106, 2538. 9, 88, 89

[88] W. Lei, A. Derecskei-Kovacs und R. Zhang, J. Chem. Phys., 2000, 113 (13), 5354-5360. 9

[89] W. Lei, R. Zhang, W. McGivern, A. Derecskei-Kovacs und S. W. North, Chem. Phys. Lett., 2000, 326, 109-114. 9, 88

[90] B. Hansmann, Die Reaktionen des Hydroxylradikals mit Propen und Isopren bei tiefen Temperaturen, Diplomarbeit, Georg-August-Universität Göttingen, 2003. 9, 42,71

[91] O. B. Toon, P. Hamill, R. P. Turco und J. Pinto, Geophys. Res. Lett., 1986, 13. 10

[92] G. Winnewisser und E. Herbst, Rep. Prog. Phys., 1993, 1209. 10

[93] E. Herbst, Ann. Rev. Chem. Phys., 1995, 46, 27. 10

[94] A. Dalgarno, J. Chem. Soc. Faraday Trans., 1993, 89, 2111. 10

[95] S. Arrhenius, Z. Phys. Chem., 1889, 4, 226. 10

[96] M. A. Smith, in C. Y. Ng und T. Baer (Hg.), "Unimolecular and Bimolecular Reaction Dynamics", Wiley, New York, 1994 183-251. 12

[97] I. W. M. Smith, in T. J. Millar und D. A. Williams (Hg.), "Rate Coefficients for Astrochemistry", Kluwer, Dordrecht, 1988 103-116. 12

[98] I. R. Sims und I. W. M. Smith, Chem. Phys. Lett., 1988, 151 (6), 481. 12

[99] M. J. Howard und I. W. M. Smith, Prog. Reac. Kinet., 1983, 12, 55. 12, 14

[100] R. Atkinson, D. L. Baulch, R. A. Cox, R. F. J. Hampson, J. A. Kerr und J. Troe, J. Phys. Chem. Ref. Data, 1992, 21, 1125. 12

[101] D. Ham, D. Trainor und F. Kaufman, J. Chem. Phys., 1970, 53, 4395. 13 
[102] D. W. Trainor, D. O. Ham und F. Kaufman, J. Chem. Phys., 1973, 58, 4599. 13

[103] B. R. Rowe, G. Dupeyrat, J. B. Marquette und P. Gaucherel, J. Chem. Phys., 1984, 80 (10), 4915-4921. 13, 41

[104] B. R. Rowe und J. B. Marquette, Int. J. Mass Spectrom. Ion Proc., 1987, 80, 239-254. 13, 41

[105] R. P. Fernando und I. W. M. Smith, Chem. Phys. Lett., 1979, 66 (2), 218-222. 14

[106] I. R. Sims und I. W. M. Smith, J. Chem Soc. Faraday Trans, 1993, 89, 1. 14

[107] R. A. Brownsword, A. Canosa, B. R. Rowe, I. R. Sims, I. W. M. Smith, D. W. A. Stewart, A. C. Symonds und D. Travers, J. Chem. Phys., 1997, 106 (18), 7662-7677. 14, 42

[108] D. Fulle, H. F. Hamann, H. F. Hippler und J. Troe, J. Chem. Phys., 1996, 105, 983. 14

[109] F. A. Lindemann, Trans. Faraday Soc., 1922, 17, 598. 17

[110] C. N. Hinshelwood, Proc. Roy. Soc. A, 1926, 113, 230. 19

[111] J. Troe, J. Chem. Phys., 1977, 66, 4745. 20

[112] J. Troe, J. Chem. Phys., 1977, 66, 4758. 20, 119

[113] J. Troe, J. Phys. Chem., 1979, 83, 114. 20

[114] M. G. Evans und M. Polanyi, Trans. Faraday Soc., 1935, 31, 875. 21

[115] H. Eyring, J. Chem. Phys., 1935, 55, 107. 21

[116] O. K. Rice und H. C. Ramsperger, J. A. Che. Soc., 1927, 49, 1617. 22

[117] L. S. Kassel, J. Phys. Chem., 1928, 32, 1065. 22

[118] R. A. Marcus und O. K. Rice, J. Phys. Chem., 1952, 55, 894. 22

[119] R. A. Marcus, J. Chem. Phys., 1966, 45, 4500. 22

[120] M. Quack und J. Troe, Ber. Bunsenges. Phys. Chem., 1974, 78, 240-252. 23

[121] M. Quack und J. Troe, Ber. Bunsenges. Phys. Chem., 1975, 79, 170-183. 23, 27

[122] J. Troe, J. Chem. Phys., 1981, 75 (1), 226-237. 23, 119 
[123] G. Z. Whitten und B. S. Rabinovitch, J. Che. Phys., 1963, 38, 2466. 26

[124] T. Beyer und D. F. Swinehart, Comm. Assoc. Comput. Machines, 1973, 16, 379. 27

[125] E. E. Nikitin, Theor. Exp. Chem., 1965, 1, 83, 90, 275. 27

[126] P. Pechukas und J. C. Light, J. Chem. Phys., 1965, 42, 3281. 27

[127] E. Schrödinger, Ann. Physik, 1926, 79, 361. 29

[128] M. Born und J. R. Oppenheimer, Ann. Physik, 1927, 84, 457. 29

[129] A. Szabo und N. S. Ostlund, Modern Quantum Chemistry, Dover Publications, Mineola, 1996. 30

[130] J. B. Foresman und A. Frisch, Exploring Chemistry with Electronic Structure Methods, 2 Aufl., Gaussian, Pittsburgh, 1996. 30

[131] D. R. Hartree, The Calculation of Atomic Structures, John Wiley \& Sons, Chichester, 1957. 31

[132] V. Fock, Zeitschr.f. Physik, 1930, 61, 126. 31

[133] C. Møller und M. S. Plesset, Phys. Rev., 1934, 46, 618. 32

[134] P. Hohenberg und W. Kohn, Phys. Rev. B, 1964, 136, 864. 33

[135] W. Kohn und L. J. Sham, Phys. Rev. A, 1965, 140, 1133. 33

[136] A. D. Becke, Phys. rev. A, 1988, 38, 3098. 34

[137] A. D. Becke, J. Chem. Phys., 1993, 98, 1372. 34

[138] A. D. Becke, J. Chem. Phys., 1993, 98, 5648. 34

[139] C. Lee, W. Yang und R. G. Parr, Phys. Rev. B, 1988, 37, 785. 34

[140] S. H. Vosko, L. Wilk und M. Nusair, Can. J. Phys., 1980, 58, 1200. 34

[141] I. W. M. Smith, Int. J. Mass Spectrom. Ion Proc., 1995, 149/150, 231. 39

[142] B. R. Rowe, J. B. Marquette und C. Rebrion, J. Chem. Soc. , Faraday Trans., 1989, 80, 1631. 41

[143] I. R. Sims, J.-L. Queffelec, D. Travers, B. R. Rowe, L. B. Herbert, J. Karthäuser und S. I. W. M., Chem. Phys. Lett., 1993, 211 (4,5), 461-468. 42, 97, 98, 103 
[144] I. R. Sims, J.-L. Queffelec, A. Defrance, C. Rebrion-Rowe, D. Travers, P. Bocherel, B. R. Rowe und I. W. M. Smith, J. Chem. Phys., 1994, 100 (6), 4229-4241. 42

[145] D. Carty, V. Le Page, I. R. Sims und I. W. M. Smith, Chem. Phys. Lett., 2001, 344, 310-316. 42

[146] I. R. Sims, I. W. M. Smith, P. Bocherel, A. Defrance, D. Travers und B. R. Rowe, J. Chem. Soc. Faraday Trans., 1994, 90, 1473. 42, 90, 91, 97

[147] I. R. Sims, I. W. M. Smith, D. C. Clary, P. Bocherel und B. R. Rowe, J. Chem. Phys., 1994, 101, 1748. 42

[148] P. Sharkey, I. R. Sims, I. W. M. Smith, P. Bocherel und B. R. Rowe, J. Chem. Soc. Faraday Trans., 1994, 90, 3609. 42

[149] P. Bocherel, L. B. Herbert, B. R. Rowe, I. R. Sims, I. W. M. Smith und D. Travers, J. Phys. Chem., 1996, 100, 3063. 42

[150] R. A. Brownsword, S. D. Gatenby, L. B. Herbert, I. W. M. Smith, D. W. A. Stewart und A. C. Symonds, J. Chem. Soc. Faraday Trans., 1996, 92, 723. 42

[151] L. B. Herbert, I. R. Sims, I. W. M. Smith, D. W. A. Steward, A. C. Symonds, A. Canosa und B. R. Rowe, 1996, 100, 14928. 42

[152] A. Canosa, I. R. Sims, D. Travers, I. W. M. Smith und B. R. Rowe, Astron. Astrophys., 1997, 323, 644-651. 42, 92, 97, 98

[153] S. D. Le Picard, A. Canosa, B. R. Rowe, R. A. Brownsword und I. W. M. Smith, J. Chem. Soc. Faraday Trans., 1998, 94, 2889-2993. 42

[154] D. Chastaing, P. L. James und I. W. M. Smith, Faraday Discuss., 1998, 109, 165. 42,97

[155] D. Chastaing, P. L. James, I. R. Sims und I. W. M. Smith, Phys, Chem. Chem. Phys., 1999, 1 (9), 2247. 42, 97, 98

[156] D. Chastaing, S. D. Le Picard, I. R. Sims, I. W. M. Smith, W. D. Geppert, C. Naulin und M. Costes, Chem. Phys. Lett., 2000, 331, 170-176. 42

[157] W. D. Geppert, D. Reigneur, T. Stoecklin, C. Naulin, M. Costes, D. Chastaing, S. D. Le Picard, I. R. Sims und I. W. M. Smith, Phys. Chem. Chem. Phys., 2000, 2, 2873-2881. 42

[158] D. B. Atkinson und M. A. Smith, J. Phys. Chem., 1994, 98, 5797. 42, 45 
[159] D. B. Atkinson, Ph.d. thesis, University of Arizona, 1995. 42, 45

[160] D. B. Atkinson und M. A. Smith, Rev. Sci. Instrum., 1995, 66 (9), 4434. 42, 45,49

[161] D. B. Atkinson, V. I. Jaramillo und M. A. Smith, J. Phys. Chem. A, 1997, 101 (18), 3356-3359. 42

[162] A. B. Vakhtin, S. Lee, D. E. Heard, I. W. M. Smith und S. R. Leone, J. Phys. Chem., 2001, 105, 7889-7895. 42

[163] S. Lee, R. J. Hoobler und S. R. Leone, Rev. Sci. Instrum., 2000, 71 (4), 1816. 42, 97

[164] S. Lee und S. R. Leone, Chem. Phys. Lett., 2000, 329 (5-6), 443. 42

[165] A. B. Vakhtin, D. E. Heard, I. W. M. Smith und S. R. Leone, Chem. Phys. Lett., 2001, 344, 317-324. 42, 97

[166] S. Hamon, S. D. Le Picard, A. Canosa und B. R. Rowe, J. Chem. Phys., 2000, 112, 4506-4516. 42

[167] S. P. K. Koehler, Low Temperature Reaction Kinetics and Dynamics of the Hydroxyl Radical, Diplomarbeit, Georg-August-Universität Göttingen, 2002. 42

[168] P. L. James, I. R. Sims und I. W. M. Smith, Chem. Phys. Lett., 1997, 276, 423-429. 42

[169] P. L. James, I. R. Sims, I. W. M. Smith, M. H. Alexander und M. A. Yang, J. Chem. Phys., 1998, 109 (10), 3882. 42

[170] S. M. A. Wright, I. R. Sims und I. W. M. Smith, J. Phys. Chem., 2000, 104, 10 347-10 355. 42

[171] A. Hasson, C. M. Moore und I. W. M. Smith, Int. J. Chem. Kinet., 1998, 30, 541-554. 42

[172] A. Bonnamy, R. Georges, A. Benidar, J. Boissoles, A. Canosa und B. R. Rowe, J. Chem Phys., 2003, 118, 3612-3621. 42, 103

[173] Y. Okada, S. Isomura, S. Satooka und K. Takeuchi, Rev. Sci. Instrum., 1996, 67 (9), 3070-3072. 42

[174] Y. Okada, S. Kato, S. Satooka und K. Takeuchi, Appl. Phys. B: Lasers Opt., 1996, 62, 515. 42 
[175] S. Tanimura, Y. Okada, Y. Kuga und K. Takeuchi, Chem. Phys. Lett., 1995, 232 (1), 176-180. 42

[176] S. Tanimura, Y. Okada und K. Takeuchi, J. Phys. Chem., 1996, 100 (8), 2842-2848. 42

[177] S. Tanimura, Y. Okada und K. Takeuchi, J. Chem. Phys., 1997, 10 r (18), 7096-7105. 42

[178] T. V. Marter, M. C. Heaven und D. Plummer, Chem. Phys. Lett., 1996, 260 (2), 210-207. 42

[179] T. V. Marter und M. C. Heaven, J. Chem. Phys., 1998, 109 (21), 9266-9271. 42

[180] T. S. Dibble und L. S. Bartell, J. Phys. Chem, 1992, 96 (5), 2317-2322. 42

[181] J. Huang, W. Lu und L. S. Bartell, J. Phys. Chem., 1996, 100 (34), $14276-$ 14280. 42

[182] G. L. Vaghjiani und A. R. Ravishankara, J. Chem. Phys., 1990, 92 (2), 9961003. 54

[183] G. L. Vaghjiani, A. A. Turnipseed, R. F. Warren und A. R. Ravishankara, J. Chem. Phys., 1992, 96 (8), 5878-5886. 54

[184] B. A. Thompson, P. Harteck und R. R. Reeves, J. Geophys. Res., 1963, 68, 6431. 54

[185] R. Atkinson, D. L. Baulch, R. A. Cox, R. F. Hampson, Jr., J. A. Kerr und J. Troe, J. Phys. Chem. Ref. Data, 1992, 21, 1174. 54

[186] T. Nakayama und K. Watanabe, J. Chem. Phys., 1964, 40, 558. 54

[187] C. Y. R. Wu, T. S. Chien, G. S. Liu, D. L. Judge und J. J. Caldwell, J. Chem. Phys., 1989, 91, 272. 54

[188] G. H. Dieke und H. M. Crosswhite, J. Quant. Spectrosc. Radiat. Transfer, 1962, 2, 97-199. 58

[189] J. A. Dodd, S. J. Lipson und W. A. M. Blumberg, J. Chem. Phys., 1991, $95(8), 5752.67$

[190] K. J. Rensberger, J. B. Jeffries und D. R. Crosley, J. Chem. Phys., 1989, $90(4), 2174.67$

[191] M. M. Ahern und S. M. A., J. Chem. Phys., 1999, 110 (17), 8555. 67 
[192] D. R. Crosley, in J. R. Barker (Hg.), "Progress and problems in atmospheric chemistry", Bd. 3, World Scientific Publishing, Singapore, 1995 256-317. 67

[193] D. A. V. Kliner und R. L. Farrow, J. Chem. Phys., 1999, 110 (1), 412. 67

[194] M. T. Berry, R. A. Loomis, L. C. Giancario und M. I. Lester, J. Chem. Phys., 1992, 96, 7890. 67

[195] M. Soerensen, E. W. Kaiser, M. D. Hurley, T. J. Wallington und O. J. Nielsen, Int. J. Chem. Kinet., 2003, 35, 191-197. 79, 80, 82

[196] R. Atkinson, J. Phys. Chem. Ref. Data, 1997, 26 (2), 215-219. 85, 87

[197] J. C. Hansen und J. S. Fransisco, Chem. Phys. Chem., 2002, 3, 833-840. 93

[198] D. L. Singleton und R. J. Cvetanovic, J. Am. Chem. Soc., 1976, 98 (22), 6812-6819. 93

[199] D. C. Clary, Ann. Rev. Phys. Chem., 1990, 41, 61. 98

[200] D. C. Clary, T. S. Stoecklin und A. G. Wickham, J. Chem. Soc. Faraday Trans., 1993, 79, 2185. 98

[201] U. Wachsmuth, Quantitative Cavity-Ringdown-Spektroskopie zur Messung von druckabhängigen Linienbreiten im Ozon, Dissertation, Georg-AugustUniversität Göttingen, 2001. 102

[202] T. Knöppel, Spektroskopie und intramolekulare Dynamik des NH2OH, Diplomarbeit, Georg-August-Universität Göttingen, 2000. 102

[203] M. J. Frisch, G. W. Trucks, H. B. Schlegel, G. E. Scuseria, M. A. Robb, J. R. Cheeseman, V. G. Zakrzewski, J. A. Montgomery, Jr., R. E. Stratmann, J. C. Burant, S. Dapprich, J. M. Millam, A. D. Daniels, K. N. Kudin, M. C. Strain, O. Farkas, J. Tomasi, V. Barone, M. Cossi, R. Cammi, B. Mennucci, C. Pomelli, C. Adamo, S. Clifford, J. Ochterski, G. A. Peterson, P. Y. Ayala, Q. Cui, K. Morokuma, D. K. Malick, A. D. Rabuck, K. Raghavachari, J. B. Foresman, J. Cioslowski, J. V. Ortiz, B. B. Stefanov, G. Liu, A. Liashenko, P. Piskorz, I. Komaromi, R. Gomperts, R. L. Martin, D. J. Fox, T. Keith, M. A. Al-Laham, C. Y. Peng, A. Nanayakkara, C. Gonzales, M. Challacombe, P. M. W. Gill, B. Johnson, W. Chen, M. W. Wong, J. L. Andres, C. Gonzales, M. Head-Gordon, E. S. Replogle und J. A. Pople, "Gaussian 98, Revision A.5", 1998. 105

[204] M. W. Chase, jr., J. Phys. Chem. Ref. Data, Monograph 9, 1998, 1-1951. 107, 109, 113 
[205] E. J. Feltham, M. J. Almond, G. Marston, K. S. Wiltshire und N. Goldberg, Spectr. Act. A, 2000, 56A (13), 2589-2603. 115

[206] J. Troe, J. Chem. Phys., 1983, 79, 6017. 119

[207] J. Troe, Ber. Bunsenges. Phys. Chem., 1983, 87, 161. 119

[208] J. Troe, Ber. Bunsenges. Phys. Chem., 1983, 87, 169. 119 



\section{Danksagung}

Zuerst möchte ich Herrn Prof. Dr. J. Troe für die freundliche Aufnahme in seine Arbeitsgruppe und die Bereitstellung hervorragender Arbeitsmöglichkeiten danken, auch wenn er manches Mal, speziell gegen Ende des Jahres, die Rute auspackt!

Ganz besonderer Dank gilt Prof. Dr. B. Abel, der durch seine stetige Betreuung und Hilfsbereitschaft sehr viel zu dieser Arbeit beigetragen hat. Dabei haben vor allem seine immerwährende Erreichbarkeit und seine Engelsgeduld, mit der er auf die ein oder anderen Ergebnisse gewartet hat, sehr geholfen.

Weiterhin möchte ich Uwe Wachsmuth danken, der mit seiner Erfahrung im wissenschaftlichen Arbeiten das Projekt Lavaldüse im Anfangsstadium angeschoben hat und die ersten Signale aus der Apparatur gekitzelt hat. Nach einer mageren Zeit einsam im Labor wurde ich mit einem Diplomanden beglückt, der das Pferd so richtig zum Laufen gebracht hat, ASR Sven Koehler (Golden Goal in Istanbul?). Auch sein Nachfolger, Björn Hansmann, konnte die CRESU-Appparatur noch entscheidend verbessern und war Partner in zahlreichen Diskussionen. Das jüngste Mitglied der CRESU-Crew, Rosalin Karunanandan, hat ebenfalls sehr zum Gelingen dieser Arbeit beigetragen, indem sie die Qualität der Messwerte nochmals verbessern konnte. Danke.

Meinem Mitinsassen Dr. rer. microl. Jens Aßmann gebührt nicht nur Dank für seine stete Hilfsbereitschaft in allen Bereichen, sondern auch für die exzellente Betreuung von Spike in meiner Abwesenheit im Schreibraum (Achtung Hund!!!). Für die kompetente Betreuung eines Computerlegasthenikers haben sich Jörg Hahn und Christian Grimm ein großes Lob verdient.

Zum akribischen Korrekturteam dieser Arbeit konnte ich glücklicherweise Rebekka von Benten, Björn Hansmann und Jörg Hahn zählen. Die weniger wissenschaftlichen Diskussionen und Themenabende mit Marco Seidel möchte ich hier noch erwähnen, sein Fachwissen auf seinem Spezialgebiet (N. P.) hat allerdings wenig zum Gelingen 
dieser Arbeit beitragen können. Allen anderen Trojanern möchte ich für ihre Anteilnahme an technischen Problemen sowie einfach nur für ihre Anwesenheit danken (Musikquiz mit Jochen!).

Das Herzstück der CRESU-Apparatur, die verschiedenen Lavaldüsen, wurden in der hauseigenen Werkstatt unter der Leitung von Herrn Meyer gefertigt, der stets zusammenzuckte, wenn ich mit dem Auftrag für eine neue, noch längere, aber mit kleinerem Durchmesser versehene Lavaldüse bei ihm hereinschneite. Dank gebührt auch der ELO für die Herstellung und die Reparatur sämtlicher kleiner und großer mit Widerständen vollgestopften Kästchen. Für alle sonstigen kleineren und größeren Wehwehchen bin ich W. Noack alias Milo und Excimer-Man R. Bürsing sehr zu Dank verpflichtet.

Meiner gesamten Familie möchte ich für Unterstützung jeder Art danken. Yvonne, danke für dein Verständnis, ich liebe dich. 


\section{Lebenslauf}

Am 28.07.1975 wurde ich, Tim Spangenberg, geb. Knöppel, als Sohn von Gabriele Knöppel, geb. Urban, und Holger Knöppel in Kassel geboren. Nach dem Besuch der Grundschule in Niedervellmar von 1981 bis 1985 wechselte ich auf das Gymnasium Albert-Schweitzer-Schule in Kassel, auf dem ich im Juni 1994 die allgemeine Hochschulreife erlangte. Von Oktober 1994 bis Oktober 1995 leistete ich meinen Zivildienst bei der Johanniter-Unfall-Hilfe e. V. in Kassel ab.

Im Wintersemester 1995/96 immatrikulierte ich mich an der Universität-Gesamthochschule Kassel, an der ich im Oktober 1997 die Diplomvorprüfung bestand. Zum Sommersemester 1998 wechselte ich an die Georg-August-Universität, ab 1999 begann ich meine Diplomarbeit im Arbeitskreis von Prof. Dr. J. Troe mit dem Thema „Spektroskopie und intramolekulare Dynamik des Hydroxylamin“. Im Juni 2000 erwarb ich das Diplom der Fakultät für Chemie Göttingen.

Anschließend begann ich im Rahmen meiner Doktorarbeit im Arbeitskreis von Prof. Dr. J. Troe Studien zu Reaktionen des Hydroxylradikals bei tiefen Temperaturen. Vom 01.01.2001 bis 31.12.2002 war ich Stipendiat der Stiftung ,Stipendien-Fonds des Verbandes der chemischen Industrie“. Ab 01.01.2003 war ich Angestellter des Max-Planck-Instituts für biophysikalische Chemie in Göttingen, ab 01.07.2003 bin ich innerhalb des Sonderforschungsbereiches 357 ,Molekulare Mechanismen unimolekularer Reaktionen" als wissenschaftlicher Mitarbeiter beschäftigt. Als Ergebnis dieser Arbeit entstand die vorliegende Dissertation.

Am 09.07.2003 heiratete ich Yvonne Spangenberg, am 24.09.2003 wurde unsere Tochter Nele Lia geboren. 